MY HISTORY IS NOT MINE

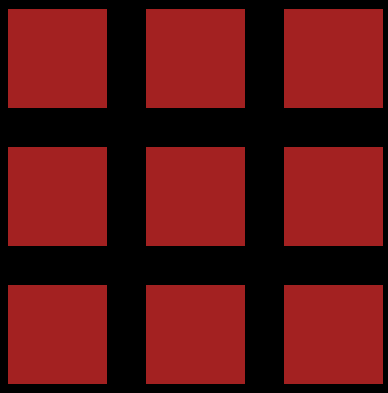

M a y M y $\quad$ M i n 
曲 


\title{
MY HISTORY IS NOT MINE
}

A Speculative Allegorical Approach to Experiential Architecture

\author{
May Myo Min
}

a 120-point thesis submitted to the Victoria University of Wellington in partial fulfillment of the requirements for the degree of Master of Architecture (Professional)

Victoria University of Wellington

School of Architecture

2020 
Abstract

\begin{abstract}
Globalisation has helped spread Eurocentric $\checkmark$ modernist architectural principles across most cultures. In a very real sense, many Eastern cultures are having their own unique architectural histories rewritten, even erased, and in danger of becoming lost. Burmese poet Zeyar Lynn's poem "My History is Not Mine" represents a powerful lament, decrying the loss of unique cultural identities. Global contemporary architecture rarely recognises the rich litany of ideas that may arise from contemporary responses to cultures, and this design research-led thesis investigation seeks possible solutions to this loss.

This investigation is framed around Lynn's poem "My History is Not Mine". It seeks to reinterpret some of the most 'traditional' elements of modern architecture-room, wall, ceiling, floor, threshold, window, etc.-through fictional narrative theory, allegory and experiential constructs. Eastern superstitions are used as provocateurs, starting points that help the project explicitly move away from traditional Eurocentric formalist architectural precepts. The goal is to test an architectural design method that prioritises the experiential and challenges some of the expected 'norms' within
\end{abstract}

which Eurocentric modern architecture has been traditionally situated. This investigation is grounded in speculative architectural design. The three principal design stages of the methodology progress iteratively from physical analogue model, to digital animation, and finally to virtual gaming environment. The intention is to challenge traditional notions of architecture and the way architectural design concepts are conceived, and this is carried forward using a methodology that shifts experimental outcomes from the visual to the experiential-a virtual, time-based approach that deviates from conventional architectural design processes-in order to privilege the investigation of shifts in spatial conditions and experiential perceptions over time.

The first stage of the investigation was to explore the abstraction of Eastern superstitions into physical models-'allegorical artefacts'. These initial experiments were set up as a starting point to help propel the project towards a provocative and evocative pathway of discovery. By examining how these superstitions might be interpreted in a virtual gaming environment in the final stages of the investigation, the investigation challenges 
how these design interpretations can actively enable important architectural elements, such as threshold, spatial enclosure, visual axes, etc., to be redefined-placing the viewer into an experiential realm that is removed from traditional architectural referencing-and engage them as changes in spatial conditions experienced over time, rather than as primarily object-based.

The time-based design outcomes are framed, experienced and tested in relation to Jerome Bruner's theory of "The Narrative Construction of Reality". Bruner posited ten requisite steps for achieving a meaningful narrative experience for the reader of narrative fiction. Fictional narrative relies on enabling the participant to self-identify within a fictional context as a vital tool that allows the participant to navigate through the story. This design-led research investigation examines how Bruner's literary theory might be applied to an architectural experience, to help enable the experiential to become a driver for architectural design, where the participant's own self-positioning in a time-based scheme becomes a vital element in constructing a unique architectural experience. The framework synthesises the design outcomes within a narrative experience that looks to discover unique solutions to the research objectives. The investigation applies Bruner's ten constructs of narrative fiction to the architectural experience: diachronicity (relationships over time), particularity (unique cultural attributes), intentional state entailment (agency), hermeneutic composability (synecdoche), canonicity and breach (disruption of the expected), referentially (creation of new realities), genericness (changing the way a story is told), normativeness (multiplicity), contextual negotiability (cultural sensitivity and culturally negotiated meanings), and narrative accrual (collective representation).

This thesis asks:

How can experiential cultural artefacts be engaged as a conceptual framework to generate an allegorical architectural project?

How can the digital gaming interface be used to help architectural design methods better explore the experiential as a design generator? 
I am already dead,

I can say this unreservedly

It wasn't me who wrote my history

Eleven out of ten people don't know me

Historians have no idea about

My history written by historians

I have no souvenir whatsoever for sale

PhD scholars, journalists

They might have written my history

(but I don't remember it)

No stone inscription

No tomb, no bone

Not even ash

\section{I have not written my history}

They have written it for me, those academics

\section{They have written their own versions}

What they have written were mythologies sprinkled with gold dust

How many sprinkles did I amount to in there

I wasn't aware of the death pit

They have written even my own death
Amid deaths and deader deaths

I was almost death untitled

A crippled death

Even the death wasn't genuine

Even in death I was exiled

They did not fully write my supporting documents

Even in death I did not have a residence permit

They have written my death

They have written my death in air

They have... they have... they have written my death in water

I read it again... my own history

Misspellings, missed grammar

I don't even know how to pronounce the word 'vocabulary'

They have written my history

Then they have airbrushed me from history

My history has just begun

I am going to write my own history. 
My History is Not Mine ... 


\section{Preface}

Th[e] personal anecdote signifies more than a requiem for a past that cannot be recovered. It highlights the importance of spatial memories to identity.

—Christina Lee 125

M cultural identity has been a mixed blend of my birthplace, my upbringing and my constant change in geographic location. My parents are of Burmese descent. I was born in Myanmar (Burma), raised in the Cook Islands, and educated in New Zealand.

During my early childhood, my parents conveyed to me many of the superstitions that form a rich narrative belonging to my Burmese heritage. Through this thesis, I aim to delve, into the unique beliefs from my cultural roots as this blended uniqueness of cultures is important to me and the cultural identity I possess. In this day and age, our personal identities and cultural identities, which are the vital constituent parts that make us unique as individuals, can often become slowly engulfed by the ideals of the Eurocentric world. Eastern culture in particular is rapidly losing much of its rich and unique cultural identity with the influx of Western ideals and the adverse effects of globalisation.

Burmese poet Zeyar Lynn's 2012 poem "My History Is Not Mine" provides the grounding framework for this investigation. Lynn's poem is a lament, proclaiming how academics and historians write our history for us, when in fact we are in the process of writing our own history ourselves. The poem speaks of historians prescribing identity, which can lead to a loss in individualism and unique cultural identity. The last lines of the poem, "My history has just begun. I am going to write my own history," echo the voice of a defiant generation, demanding agency in choosing who they wish to be and how they wish to define themselves as unique individuals. This design-led thesis investigation invites a 
renewal of architectural agency through the use of computer gaming as an architectural design investigation medium, driven by the poem's cry for free will in the construction of one's own truth and reality. In gaming, agency allows for different experiences and outcomes for each player, and it offers a unique construct that is theirs and theirs alone. Traditional methods of designing architecture struggle to incorporate agency as an important issue needing to be addressed by the design process.

The genesis of the iterative series of design experiments in this thesis investigation embraces an approach where personal and cultural elements that are unique to my upbringing are extracted and interrogated through architectural means as generators for the thesis-a search for my personal identity that can be expressed uniquely in my architecture. The autobiographical starting points for the design research experiments are meant to simply act as generators of unique and provocative outcomes. The supernatural nature of the Burmese superstitions that are used as architectural design generators situate the project into a dreamlike realm, evoking a sense of mystery that enables each viewer to interpret them with free will, agency.
By positioning itself to be drawn from an autobiographical genesis, the thesis opens an opportunity for my own unique design signature to be explored and arise, while not becoming so personal that it can no longer be interpreted by others. The architectural vocabulary arising from the superstitions enabled important architectural elements, such as threshold, spatial enclosure, visual axes, etc., to be speculatively redefined, placing the viewer into an experiential realm, through the use of virtual gaming technology, that has often been ignored in traditional architectural design approaches. The goal of the investigation is to invite architectural design to become an experiential canvas upon which a person's unique culture can be situated and a personal anecdote applied. 
There is a need in all of us to have a place to hide or store certain memories, thoughts, impulses, hopes, and dreams. These are part of our lives that we cant resolve or best not act upon but at the same time we are afraid to jettison them. For some, this is a physical place; for others, it is a mental space, and for a few it is neither.

— Wong Kor Wii

$\mathrm{X}$ 
For me as author of this investigation, it is within the imaginative construct of this project that I can store my own memories. 

Acknowledgments

To my family, without whom this
thesis would not have come to fruition.

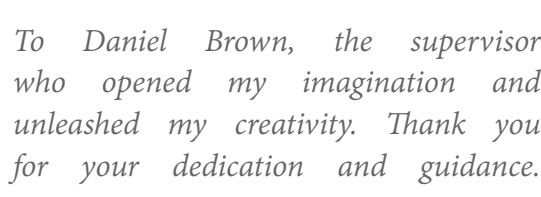


This thesis is accompanied with digital animation and game files.

Look for this symbol throughout the book.

\section{(ㄱ)}

If viewing as a book: refer to content on USB for digital files.

If viewing as a PDF: clicking on the symbols or image will direct you to a web link. 
Click to view:

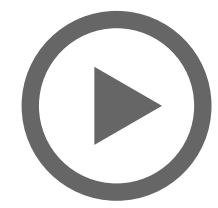

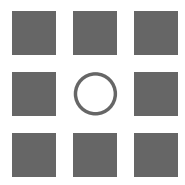




\section{Contents}

Abstract iv

Preface viii

Acknowledgments xiii

1. Introduction 1

1.1 Problem Statement

1.2 Proposition

1.3 Research Questions

1.4 Research Aim

1.5 Research Objectives

1.6 Scope

1.7 Thesis Structure

1.8 Design Research Methods and Processes

2. Literature \& Project Review

2.1 The Allegorical Architectural Project: Figurative Geometries

2.1.1 Semantic Codes

2.1.2 Autonomous neutral objects

2.1.3 Timelessness

2.1.4 Time \& Space

2.2 Narrative Architecture: Progress \& Journey

2.2.1 Narrative

2.2.2 Time-Based Event: Film And Animation

2.2.3 Chronotope

2.2.4 Removing Authorship

2.3.1 Agency 
2.3 Experiential Architecture: Invitation for Interpretation

2.3.2 The Individual's Experience as a Narrative

2.3.3 Digital Gaming Environment

2.3.4 The Chronogram

\section{Preliminary Design}

3.1 Design Stage 1

3.2 Design Stage 2

3.2.1 The Song Tower

3.2.2 The Sounding Summit

3.2.3 The Foyer

3.2.4 The Lucid Chamber

3.2.5 The Gateway

3.2.6 The Hinged Room

3.2.7 The Enclosure

3.2.8 The Frames of Isolation

3.2.9 The Dormant Crypt

\section{Developed Design}

4.1 Design Stage 3

\section{Conclusions \& Critical Reflections}

Cited Works

Bibliography

Sources of Figures 
曲 


\section{Introduction}

They have written my history

Then they have airbrushed me from history

My history has just begun

I am going to write my own history.

—Zeyar Lynn, 2012

\subsection{PROBLEM STATEMENT}

Modernist architectural principles have traditionally been dominated by Western ideals and Eurocentric constructs. Globalisation has helped spread modernist architectural principles across most cultures. When contemporary architecture seeks to respond to cultural contexts, it unfortunately often resorts to borrowing from vernacular traditions and adapting them to conform to Eurocentric modernist ideals.

This design research-led thesis investigation seeks possible speculative solutions that can help mitigate the loss of unique cultural identity in architecture and encourage opportunities beyond simply conforming to conventional methods of architectural design and traditional Eurocentric formalist architectural principles. 


\subsection{PROPOSITION}

This design-led research investigation is framed around Burmese poet Zeyar Lynn's poem "My History is Not Mine", which represents a powerful lament, decrying the loss of unique cultural identities. The investigation seeks to reinterpret some of the most 'traditional' elements of modern architecture through fictional narrative theory, allegory and experiential constructs. Eastern superstitions are used as provocateurs, starting points that help the project explicitly move away from traditional Eurocentric formalist architectural precepts. The goal is to speculatively test an architectural design method that prioritises the experiential and challenges some of the 'norms' within which Eurocentric modern architecture have been traditionally situated, e.g., to reinterpret the notion of the threshold as more than a door, and a room as more than a contained box, to name a few. This investigation is grounded in speculative architectural design that introduces the experiential (time) and spatial relationships as important factors in the reinterpretation of these elementsassessing how they can become redefined through shifts in spatial conditions as events occurring over time. It questions how architecture can find ways to defy presumptive norms-both in relation to cultural archetypes as well as methods of inquiry about architectural form, experience and space. 


\subsection{RESEARCH QUESTIONS}

This thesis asks:

How can experiential cultural artefacts be engaged as a conceptual framework to generate an allegorical architectural project?

RQ 2 How can the digital gaming interface be used to help architectural design methods better explore the experiential as a design generator? 


\subsection{RESEARCH AIM}

The principal Research Aim of the investigation is :

RA

To test an architectural design method that prioritises the experiential and challenges some of the 'norms' within which Eurocentric modern architecture have been traditionally situated.

\subsection{RESEARCH OBJECTIVES}

The principal Research Objectives of the investigation are :

RO 1

To explore how culturally scripted ideas can be expressed through allegorical methods in the conception of an evocative architectural framework that deviates from Eurocentric modernist principles and constructs.

To explore how narrative architecture can

RO 2 activate allegory within the experiential as a tool for reinterpreting notions of 'traditional' architectural elements through shifts in spatial conditions over time.

RO 3 To explore how Digital Gaming Environments can be actively engaged in the architectural design process to enable agency to actively participate in the experiential narrative outcomes. 


\subsection{SCOPE}

This design research investigation is speculative in nature. It draws from Eastern superstitions as provocative generators, starting points to drive the design experiments. The nine selected traditional Burmese superstitions were the most common ones involved in my own personal upbringing, and they provided a rich and complex foundation for the genesis of the design research experiments.

This investigation takes a speculative and allegorical approach to unveiling cultural concepts within a configuration of architectural artefacts in the context of a virtual realm. It prioritises the theoretical, and architecture for the real world of construction is outside the scope of the investigation. The project challenges how important architectural elements, such as threshold, spatial enclosure, visual axes, etc., can be redefined by placing the viewer into an experiential realm that is removed from traditional architectural referencing. As such, the bounds of an actual site context, physical limitations of a building envelope, construction costs, traditional programme and engineering technical requirements are all outside the scope of the investigation.
While this research preferences the experiential, it acknowledges that the principal use of a gaming environment places phenomenological experience beyond the scope of the investigation, except for sight and sound. The author acknowledges that it is not only vision and sound that are vital to the experience of space; the sense of touch, smell and the presence of the physical body interacting in the space (the haptic) open up interpretations that are impossible in a gaming environment. This thesis acknowledges this limitation, and these factors are outside of the scope of the investigation.

Due to constraints relating to time, resource limitations and technical capability, the digital realm was a natural choice for me in selecting how to best explore and simulate the notions of timebased events and generate infinite possibilities of outcome and interpretation, through the shifting perception and agency represented by the diverse persons who might enter the gaming environment. 
1.7 THESIS STRUCIU
Introduction

$=$

Literature and Project Review

$=$ 


\section{- THE ALLEGORICAL ARCHITECTURAL PROJECT: FIGURATIVE GEOMETRIES}

Allegory in literature is the structure of thought where metaphorical construct gives the story meaning. Allegory offers unique and creative design opportunities for conveying metaphorical meaning in works that contain encrypted ideas, where the ideas are either best left unrevealed or do not wish to be revealed. It offers a meaningful layer upon which complex thoughts and ideas can be founded, as it acts as the provocateur that drives the design motive towards a meaningful and unique direction.

RO 1: To explore how culturally scripted ideas can be explored and expressed through allegorical methods in the conception of an evocative architectural framework that deviates from Eurocentric modernist principles and constructs.

\section{Principal Theorists: Penelope Haralambidou, Angus Fletcher, Roland Barthes}

These theorists contribute to the theory of the critical role of allegory in the construction of an architectural conceptual framework that is scripted with cultural concepts. Haralambidou discusses the figurative geometries as a trait of allegory founded on the views of literary critic, Angus Fletcher. The figurative geometries are rooted in the theory of semiotics founded upon Roland Barthes's theory of the five semantic codes.

\section{Principal Case Study: Bernard Tschumi's Parc de la Villette}

Tschumi's la Villette is an example of 'figurative geometries' that are conceived through a denotation system that renounces being classified within an architectural lineage, allowing the architecture to be defined by the people and events that occupy it at that specific moment. This case study is helpful, as it represents architecture that is not based on 'traditional' elements of modern architecture-room, wall, ceiling, floor, etc. Its limitations are that it is not culturally scripted; however it does draw ideas from unconventional starting points and thus deviates from 'traditional' architectural approaches. 


\section{- NARRATIVE ARCHITECTURE: PROGRESS AND JOURNEY}

Once an allegorical framework is established for an architectural narrative, the experiential needs to be incorporated-by incorporating time and shifting spatial conditions. Penelope Haralambidou refers to this requirement as 'Progress and Journey'. Narrative theory acts as a framework in this design investigation that synthesises the formal with the experiential. Narrative theory actively participates in the construction of the architectural experience by assessing it through a framework that sets up criteria for constructing fictional narratives. It adds an additional opportunity to challenge the traditional ways of understanding and conceiving architectural preceptsby conceiving the construct of architectural space as redefined by the architectural narrative experience.

RO 2: To explore how narrative architecture can activate allegory within the experiential as a tool for reinterpreting the notions of 'traditional' architectural elements through their shift in spatial conditions over time.

\section{Principal Theorists: Jerome Bruner, Cathy Ganoe, Anthony Vidler}

The role of narrative theory in the construction of animated schemes is adopted as a vital method of challenging architectural precepts by introducing time as a factor that shifts spatial conditions and unearths new spatial outcomes. Jerome Bruner lists ten features that are requisite to constructing a fictional narrative, and Cathy Ganoe reinterprets Bruner's ten components in the context of space-to argue that the nature of narrative as a method of storytelling can be applied in the field of architecture as well. Vidler discusses the role of film in architecture to construct a narrative experience using some of the elements of Bruner's criteria, enabling the thesis investigation to draw from architectural, graphic and film conventions.

\section{Principal Case Study: John Hejduk's Chronotopes}

A selection of Hejduk's chronotopes are useful case studies in this investigation, as they are types of autonomous architectonic figures devised using concepts of time and shifting spatial conditions. The elements shift in space over time. The masques are also useful to look at as they also relate to the other two Research Objectives as well: they contain scripted ideas; they are autonomous in the way that they do not conform to any given period of time and/or culture; they convey elements of a set of game-like characters within a constructed reality; and the masques often require the participant's own perceptive experience to be able to find meaning in it. 


\section{- EXPERIENTIAL ARCHITECTURE: INVITATION FOR INTERPRETATION}

Once an allegorical architectural framework engages the experiential, agency and cultural sensitivity/negotiability must be incorporated to attain a meaningful narrative experience. The virtual gaming environment introduces agency as a personalised experience that further challenges the conventional ways that architectural design is perceived. By enabling individually selected shifts in perception, the elements of architecture in question can be further redefined through the diverse perceptions of various participants. The construct of the spatial experience is assessed against the theory of Jerome Bruner's "The Narrative Construction of Reality" to interrogate how the experience can be a meaningful encounter for diverse participants.

RO 3: To explore how Digital Gaming Environments can be actively engaged in the architectural design methods and processes to enable agency to actively participate in the experiential narrative outcomes.

\section{Principal Theorists: Jerome Bruner, Cathy Ganoe, Sophia Psarra}

Bruner's narrative theory discusses three features that contribute to the construct of agency within the Digital Gaming Environment that are vital to constructing a personalised experiential narrative. Intentional state entailment, normativeness and context sensitivity/negotiability are the three main features that are discussed to enable the element of agency. Cathy Ganoe plays a role in redefining Bruner's theory within an architectural spatial context, and she also discusses the interpretation of space through an individual's own unique experience and perception. Sophia Psarra critically reflects on the role of agency where interpretation needs to be perceived by a participant both in fictional narrative (as a reader) as well as in a spatial context (as a viewer).

\section{Principal Case Study: Nic Clear's Chronogram}

The digital gaming environment is reinterpreted as a form of a chronogram (Greek for 'time writing') - a spatial medium that illustrates and captures concepts of space and time-as developed by Nic Clear. This case study is useful as the chronogram does what the thesis's Digital Gaming Environment sets out to do: combine architectural, graphic and film conventions and synthesise the constituent elements by mapping out the formal, narrative, experiential and spatial possibilities of the project. The conclusion is that the Digital Game Environment is a form of a chronogram. 


\section{Preliminary Design}

\section{Design: Stage 1 [Physical Models]}

The first section of the Preliminary Design chapter represents Stage 1 of the design investigation. It explores means of addressing RO 1: To explore how culturally scripted ideas can be explored and expressed through allegorical methods in the conception of an evocative architectural framework that deviates from Eurocentric modernist principles and constructs. Stage 1 uses physical models to set out preliminary design experiments that explore the ideas developed in the Literature and Project Review chapter in relation to the Research Questions. This chapter develops an evocative conceptual framework using physical models derived from the abstraction of nine Eastern superstitions as allegorical artefacts. Each design experiment is then reflected upon and analysed to identify how it might further address the three principal Research Objectives by exploring them as digital animations in Stage 2 .

\section{Design: Stage 2 [Digital Animations]}

The second section of the Preliminary Design chapter represents Stage 2 of the design investigation. It explores means of addressing $\mathrm{RO} 2$ : To explore how narrative architecture can activate allegory within the experiential as a tool for reinterpreting the notions of 'traditional' architectural elements through shifts in spatial conditions over time. Stage 2 examines and further develops the preliminary design experiments from Stage 1. Stage 2 translates the Stage 1 analogue models (allegorical artefacts) into 3-dmensional digital models, and tests them as time-based animations to help reinterpret traditional elements within architecture-room, wall, floor, threshold, light, etc-as changes in spatial conditions through a time-based construct. 


\section{Developed Design}

\section{Design: Stage 3 [Digital Game Environment]}

This chapter represents Stage 3 of the design investigation. It explores means of addressing RO 3: To explore how Digital Gaming Environments can be actively engaged in architectural design methods and processes to enable agency to actively participate in experiential narrative outcomes. Stage 3 introduces the digital gaming environment as a medium within which the Stage 2 design outcomes can be framed, experienced and tested in relation to narrative theory. It introduces self-positioning of the participant as a vital tool for interpreting and navigating through one's own experience within the constructs of architectural spaces.

\section{Conclusions \& Critical Reflections}

This chapter critically reflects on the designs in relation to the scope of the research and disciplinary body of knowledge. It reflects upon why this has been an important issue to resolve through design; how effective the methodology was; how successful the architectural experiences are; what conclusions can be drawn from the investigation; the constraints and limitations of the findings; other potential applications of the findings; and how might this study advance further in future, if extended beyond the original scope. 


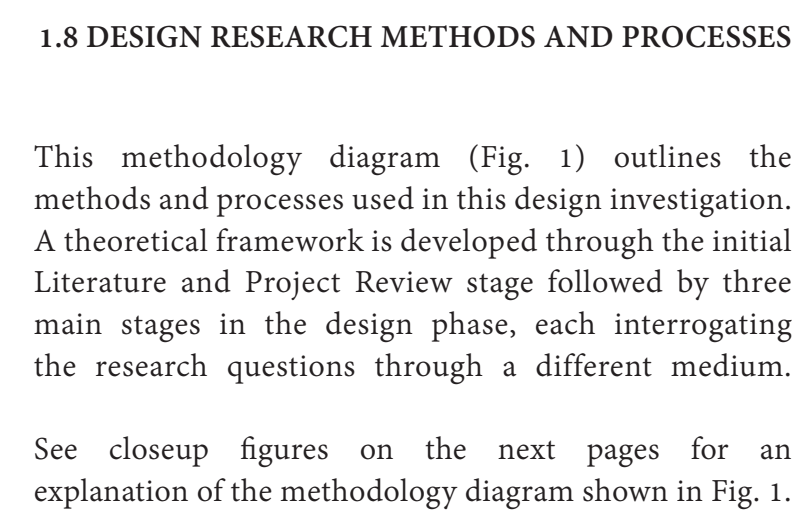




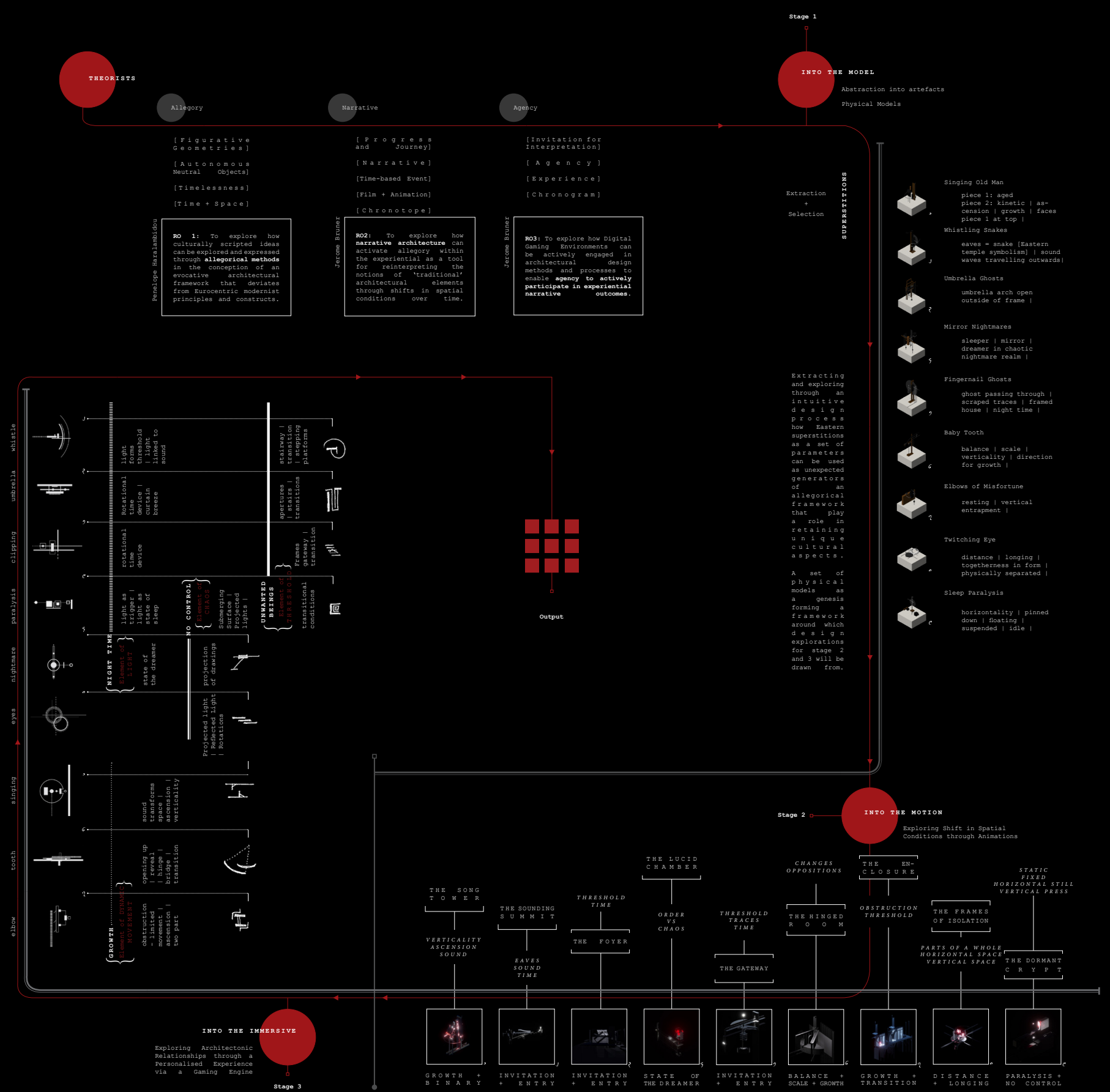

Fig. 1. Methodology Diagram 


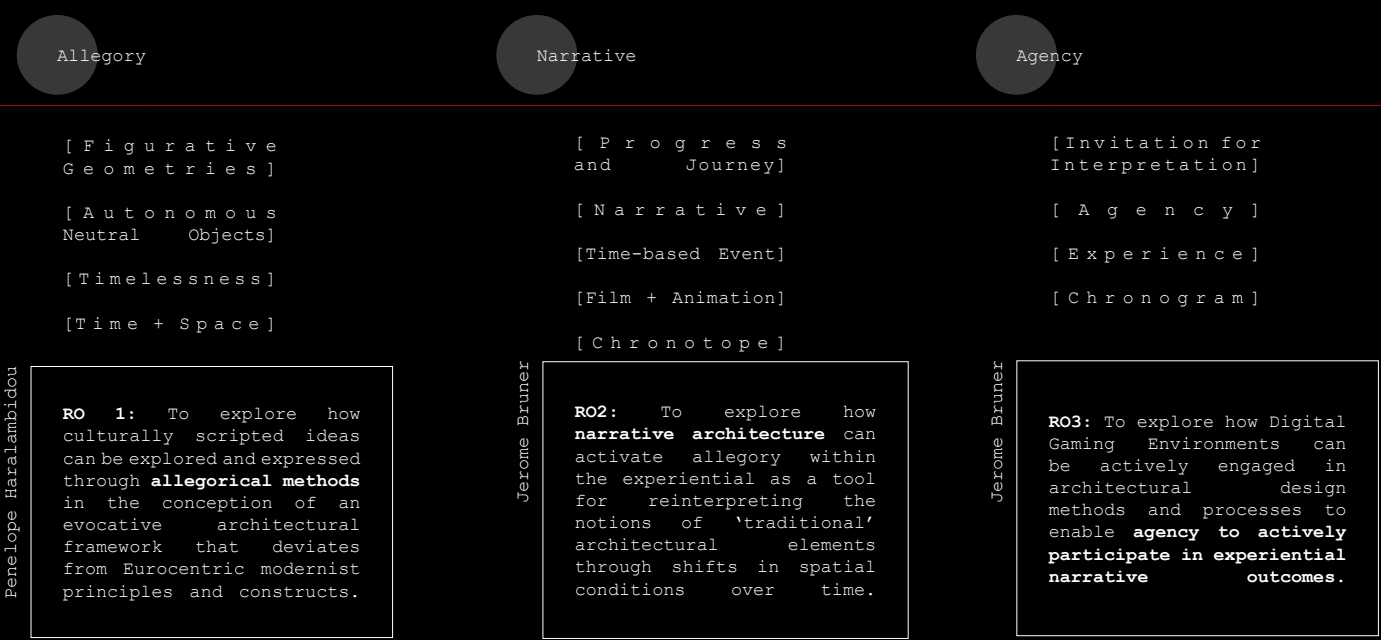

The Literature and Project Review interrogates key theorists and case studies aligned with each of the three ROs that will be interrogated through each phase of the three design stages. 
INTO THE MODEL

Abstraction into artefacts

Physical Models

The first design stage involves interpreting Eastern superstitions as design generators using physical analogue modelling through allegorical methods.

III Design Stage 1 aims to address RO1.

RO 1: To explore how culturally scripted ideas can be explored and expressed through allegorical methods in the conception of an evocative architectural framework that deviates from Eurocentric modernist principles and constructs.

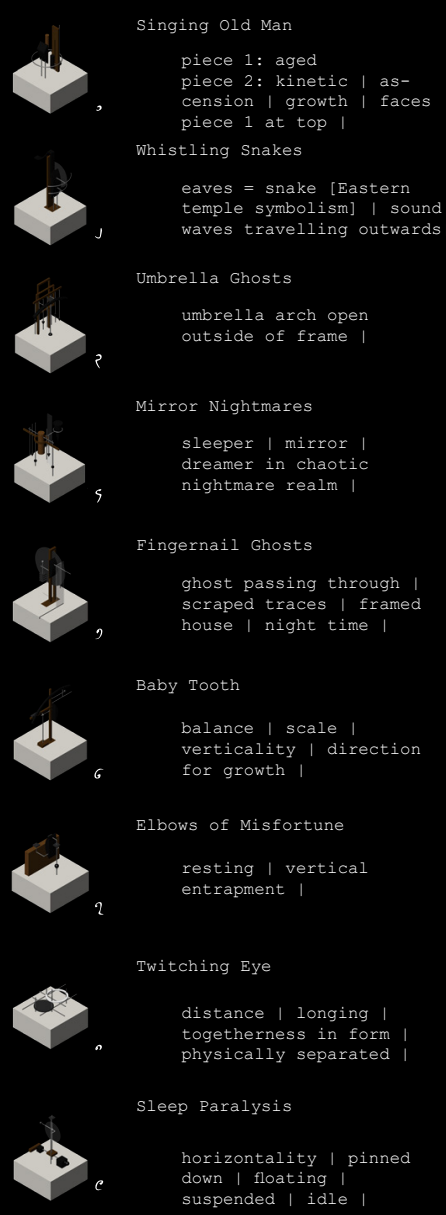

xtracting

and exploring

through an

intuitive

d e s i g $n$

p r o c e s s

Eastern

superstitions

parameters

can be used

as unexpected

of

of legorical

framework

that play

a role in

retaining

a spects.

A set of

physica 1

models as

a genesis

framework

around which

d e s i g $n$

explorations

for stage 2

and 3 will be

drawn from. 
The second stage involves testing how the physical models can be experienced relative to one another and how they can reinterpret architectural elements by using digital animations. It employs diachronicity-time-based narrative theory - to construct an architectural experience. The diagram represents the architectural elements and notions interrogated by each of the 9 animated schemes.

II Design Stage 2 aims to address RO2.
RO 2: To explore how narrative architecture can activate allegory within the experiential as a tool for reinterpreting the notions of 'traditional' architectural elements through their shift in spatial conditions over time.

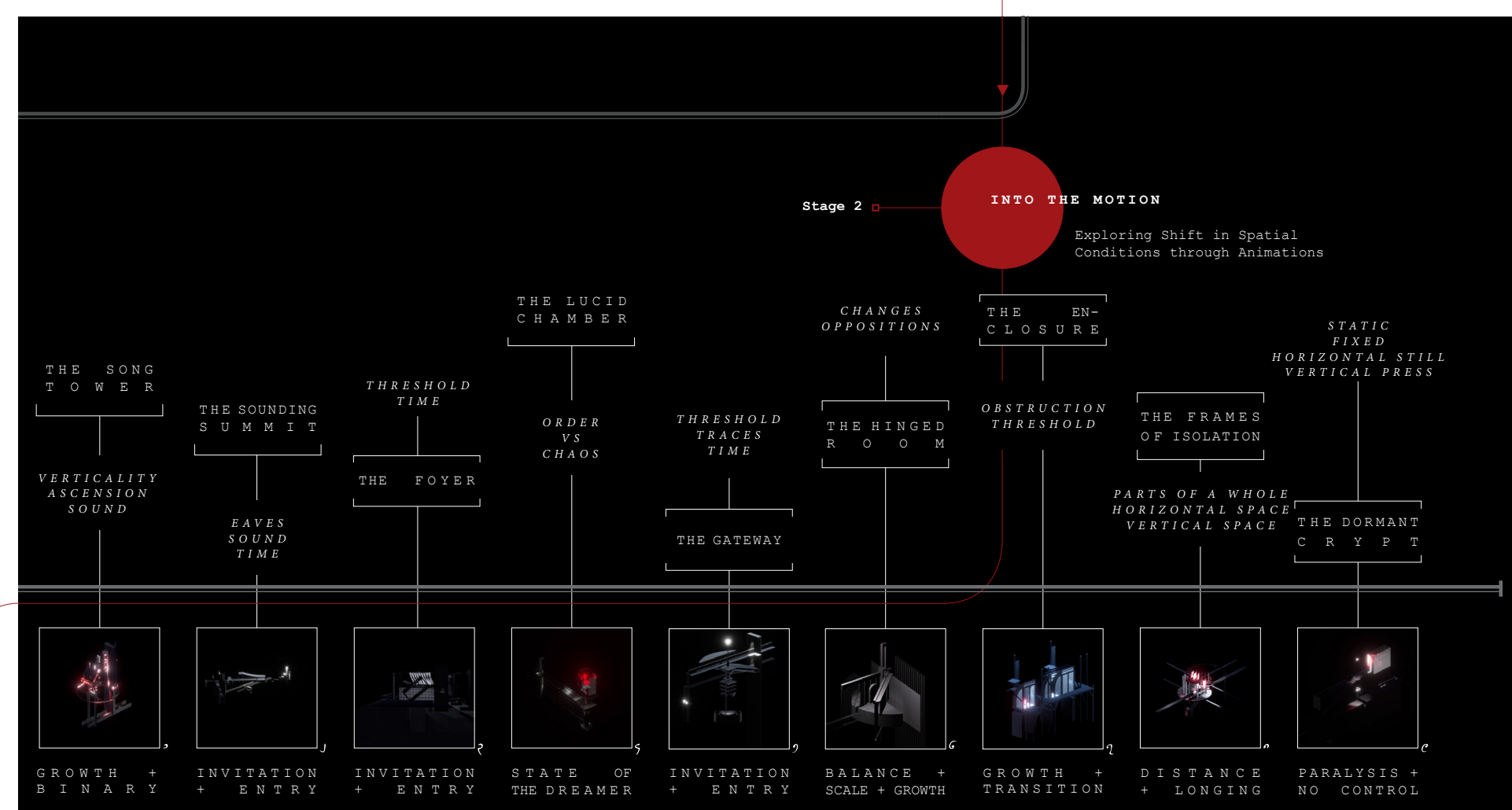




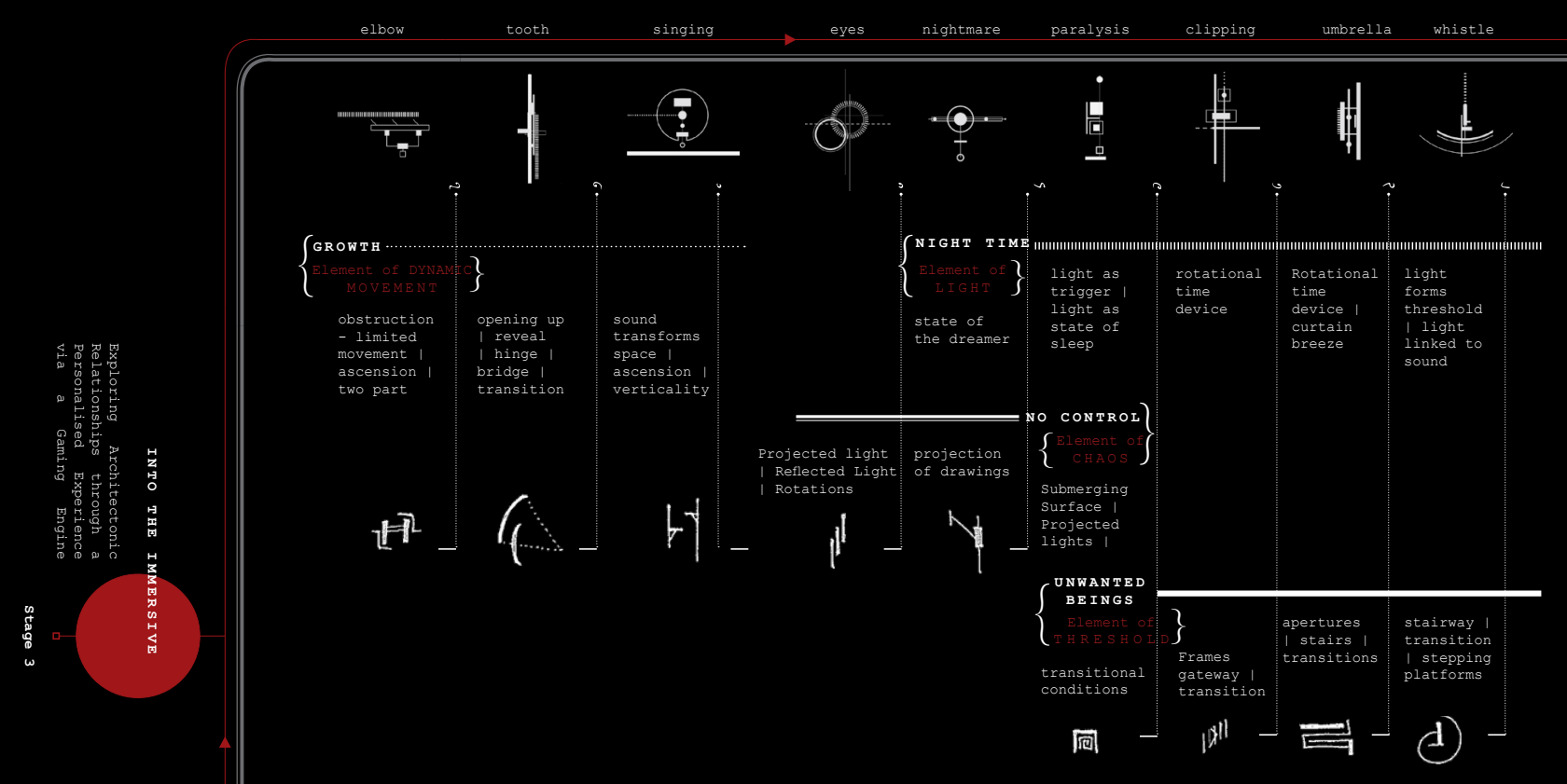

The third stage involves shifting the animated schemes into a digital game environment where the 9 interventions coexist as a collective whole. The diagram aligns each of the interventions with similar themes they share to determine how they will interact with each other and the collective whole. This stage introduces the element of agency to produce a unique personalised experience for each of the individual participants of the game.

III Design Stage 3 aims to address RO3.

RO 3: To explore how Digital Gaming Environments can be actively engaged in the architectural design methods and processes to enable agency to actively participate in the experiential narrative outcomes. 


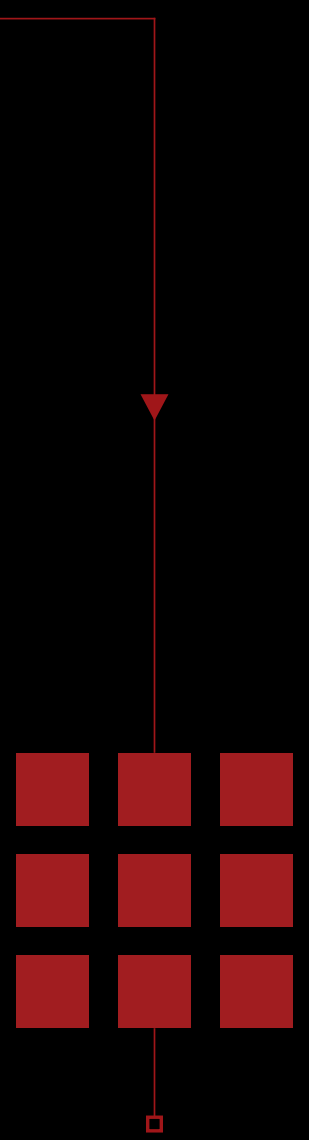

Output 
The final design stage simulates the experience of the constant shift in spatial conditions of the 9 interventions as a collective whole within a virtual experience. The outcome is framed and tested in relation to Jerome Bruner's theory of "The Narrative Construction of Reality", by situating the preliminary design experiments into the digital game environment. The three principal design stages of the methodology progress iteratively from physical analogue model, to digital animation, and finally to virtual gaming environment. The intention is to challenge traditional notions of architecture and the way architectural concepts are conceived. The investigation utilises a methodology that is situated within the experiential-a virtual time-based approach that aims to challenge conventional architectural methods and processes.

The experiments transition iteratively from Stage 1 through Stage 3,

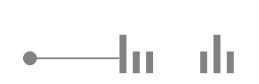

and they transform based on the learnings throughout each stage, based on the influence and capabilities of the three media. This three-stage iterative methodology reflects back to the concern over self-identity in the poem-that is, that there is no one answer, no one method, and no one factor that defines one's identity. What makes an individual unique is an array of layered traits that is theirs and theirs alone. And so the use of multiple mediums is ultimately my way of searching for answers, not just to the research questions, but also in the search for my personal artistic and unique architectural identity as author of this investigation. 
曲 


\section{Literature \& Project Review}

This chapter discusses principal theorists in relation to the three Research Objectives, and it interrogates case studies that can be interpreted as successful applications of the theories in relation to the Research Questions. The investigation explores the Allegorical Architectural Project as a framework within which architectural and spatial/experiential concepts can be reinterpreted and redefined.

The allegorical architectural project synthesises design and theory through visually creative, critical articulation of concepts. In her article "The Fall: The Allegorical Architectural Project as a Critical Method", Dr. Penelope Haralambidou, Associate Professor and Director of Communications at the Bartlett School of Architecture, writes that "the allegorical architectural project [is] an experimental practice, pointing to ideas impossible to grasp through the profession or in purely discourse-based theoretical investigations" (234). She discusses how allegory offers a critical potential in architectural design and theory. This thesis seeks to redefine conventional notions of architectural elements through speculative means and situates itself as an allegorical project that explores these challenges.
Haralambidou critically reflects upon three principal traits of allegory in architecture, and these will be discussed as contributors to the theoretical framework on allegory. The selected traits are:

\section{- Figurative Geometries \\ - progress and journey \\ - INVITATION FOR INTERPRETATION}

These three traits will be discussed in the three main sections of this chapter, to help establish a multilayered theoretical background for the development of this design-led research investigation. Figurative Geometries enable architectural narratives to engage meaning through allegory; Progress and Journey understood as a sequence of events - introduce the importance of time, experience and shifting spatial conditions in an allegorical narrative; and Invitation for Interpretation introduces the importance of agency and cultural sensitivity/negotiability within a meaningful allegorical narrative experience. 
22 


\section{Figurative Geometries}

RO 1: To explore how culturally scripted ideas can be explored and expressed through allegorical methods in the conception of an evocative architectural framework that deviates from Eurocentric modernist principles and constructs.

\section{CC}

Allegory is a structure of thought where meaning is not grasped directly but through metaphor, that often takes the guise of narrative and story-telling" (Haralambidou 226).

The first principal trait of allegory extracted from Haralambidou's writing is the notion of Figurative Geometries. This section considers Figurative Geometries in relation to Semantic Codes, Autonomous Neutral Objects, Timelessness, and Time and Space. It critically reflects on Figurative Geometries in the allegorical architectural project in relation to theorists Penelope Haralambidou, Angus Fletcher and Roland Barthes.
American literary critic Angus Fletcher describes allegory as 'figurative geometry' - the abstraction of personages and everyday objects to signify meaning and relationships (231). This notion of the figurative geometry is employed as a tool in the thesis's first design stage to generate architectural artefacts that are abstractions of Eastern superstitions; these will become provocateurs that set up a conceptual framework from which design ideas are conceived, developed and transformed. Penelope Haralambidou incorporates Fletcher's theory of figurative geometry in her article "The Fall: The Allegorical Architectural Project as a Critical Method". 


\subsubsection{SEMANTIC CODES}

To interpret a text is not to give it a (more or less justified, more or less free) meaning but on the contrary to appreciate what plural constitutes it.

—Roland Barthes 4
The theoretical foundation behind the generation of the thesis's architectural artefacts draws from French writer and critic Roland Barthes's five codes of semiotics as perspectives of viewing and interpreting text. The theory of signified meaning comes down to the study of semiotics - the study of signs and symbols and means of interpreting them. Barthes's codes imply that there is more than one way to read a text; more than one meaning can be unearthed below the surface meaning (Barthes 18). This theoretical framework is adopted to focus on the generation of the figurative geometries through Barthes's five codes: the Hermeneutic Code, the Enigma/Proairetic Code, the Symbolic Code, the Cultural Code, and the Semantic Code.

Table 1 discusses the role of each code and how they are intended to be applied in this design investigation: 


\section{The Hermeneutic Code}

The way a story drops clues to create a sense of mystery by not revealing all the truths and facts.
The metaphor and allegorical containment of meaning in the morphological development of a story.

\section{The Enigma/Proairetic Code}

The build up of tension to keep the audience guessing what might happen next.
The architectural vocabulary used: deviating away from traditional/cultural lineage and being about 'other', i.e., giving a sense of mystery and enigma.

\section{The Symbolic Code}

The way new meaning arises out of opposing and conflicting ideas, which organises semantic meanings into broader and deeper sets of meaning.
The uniting of autonomous objects that are of opposing and conflicting ideas and organising them within a system to form dialogues with one another.

\section{The Cultural Code}

The importance and influence of the audience's wider cultural knowledge, morality and ideology.
The removal of cultural implications in order for the wider audience to be able to apply their own individual ideologies into the meaning and interpretation of the space.

\section{The Semantic Code}

The way any element in a text suggests a particular meaning, by way of connotationie, the cultural/underlying meaning that it symbolises - which the story suggests.
The denotation and abstraction of superstitions to construct formal outcomes. 


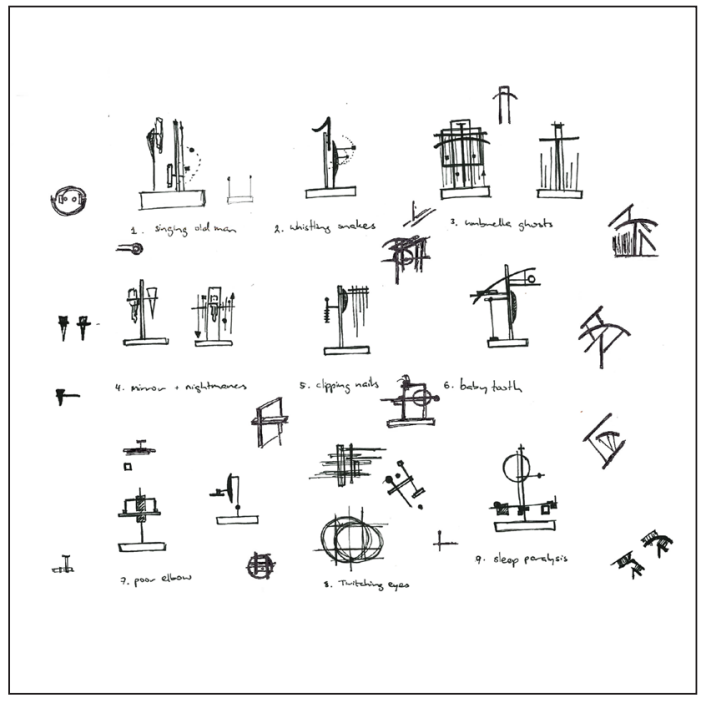

Fig. 2. Early conceptual sketch compilation of 'architectural' artefacts

Image by Author

The figurative geometry is applied in this thesis to generate a set of architectural artefacts to establish an allegorical framework through which architectural spatial concepts can be advanced (Fig. 2).

In this thesis investigation, an 'architectural artefact' implies that it is only a remnant of a whole, and it suggests belonging to cultural and/ or historic origins. The 'architectural artefact' is relevant to this thesis since the superstitions originate from a strong Eastern (Burmese) lineage, and the abstraction process has fragmented the concept to generate figurative geometriesreducing the signifier into remnants of a whole.
The Preliminary Design phase of the thesis investigation drew from all three sections of the Literature and Project Review as they were progressively developed. During the interrogation of figurative geometries, architectural artefacts were imagined and developed. The artefacts denote cultural superstitions and the abstraction process pulls apart each superstition and reconstructs formal outcomes. Barthes's five semantic codes are employed as a tool to assess the design explorations carried out in stage one of the preliminary design investigation in developing an allegorical architectural project.

Haralambidou discusses the role of allegory in an architectural project as an alternative to articulate something that is of 'other'. The works focus on a language that is autonomous-not bound to the cultural symbolism from which each one takes its roots. The works instead reflect a unique set of semantic codes that represent 'other'. The works become artefacts-nine pieces upon which the design thesis is founded and developed. The nine figurative artefacts - the abstraction of personages to signify meaning and relationships-act as the framework through which architectural spatial concepts are further explored, founded upon a set of parameters that is not of the ordinary and belonging to something that is indeed 'of other' to arrive at unexpected outcomes.

However particular its figurative elements are, the allegory remains abstract as if allegorical themes are emptied of their content by the structure that governs them. 


\subsubsection{AUTONOMOUS NEUTRAL OBJECTS}

\section{Case Study}

Bernard Tschumi's Parc de la Villette

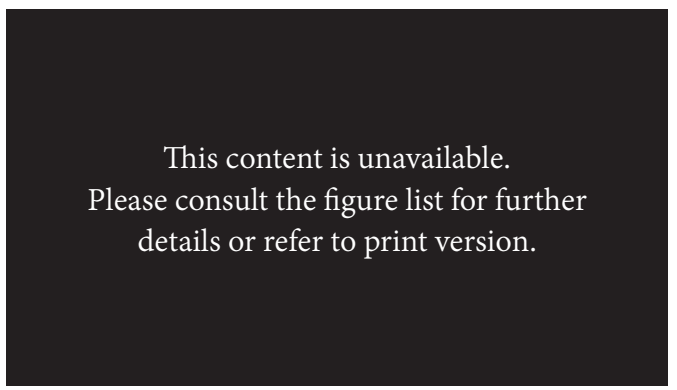

Fig. 3. Tschumi's Conceptual Notation

Diagram models of Parc de la Villette

Bernard Tschumi's Parc de la Villette can be discussed as built forms that are types of figurative geometries-denotations within which complex abstractions of movement are spatialised as a concept. Tschumi refers to them as 'folies'.
Tschumi writes in Cinegram folie: le Parc de la Villette:

The new meaning of folie [replaces] the
extravagant display of eclectic styles with the
regulated juxtaposition of unprecedented
programmes. The purpose of this operation
is to remove la folie from immersion
in the historical object and to relocate
it on the broader level of abstraction
as an autonomous neutral object (5).

The autonomous neutral objects here deviate from traditional rules of architectural composition and agenda (Fig. 3). They are formed through the process of abstract mediation and complex transformational relations. The follies exist on their own as playful artefacts, independent of each other.

Architectural historian and critic Anthony Vidler writes about Tschumi's la Villette 'folies':

On examination, the folly is, on one level, genuinely a meaningless object, a reassemblage of once-meaningful terms to make a nonsense out of them. With no hidden political agenda, no revolutionary aesthetic or social aim, and no historicist nostalgia, the allusion to constructivism becomes a mad shot in the dark that at once cherishes avant-gardism but comprehends its madness (The Architectural Uncanny 110). 


\subsubsection{TIMELESSNESS}

Tschumi's employment of abstract mediation as a methodology in la Villette produced an outcome that denies being classified within a traditional architectural lineage, allowing the architecture to be defined by the people and events that occupy it at a specific moment. La Villette is a construct of complex architectural organisations that does not resort to traditional rules of composition, hierarchy and order. The thesis finds its first particular interest in this as an underlying factor addressing the problematic nature of architecture's inability to engage with issues of its era in a timely manner that is appropriate to a unique cultural context. Belonging to no particular language of cultural origin or era, la Villette is able to be understood and interpreted as a space whether it is visited by people from the 1980 s, when it was first built, or from the $21 \mathrm{st}$ century and Europeans or non-Europeans. The space itself does not transform but the occupation and the meaning of the space transforms over time.

According to Tschumi:

Each observer will project his own
interpretation, resulting in an account that
will again be interpreted ... and so on. In
consequence, there is no absolute "truth"
to the architectural project, for whatever
"meaning" it may have is a function of the
interpretation: it is not resident in the object, or
in the object's materials (Cinegram folie viii).

This thesis searches for examples of timeless architecture that-even when based on concepts from a specific time and culture-can offer a multitude of meanings and ever-changing interpretations relating to the unique time and unique occupants experiencing the space.

The second contribution la Villette makes to the design investigation is that la Villette suggests that the experience needs to be in a constant state of flux-ever-changing through time-to achieve the notion of timeless experience. This notion is important in this design investigation where the second and third design stages interrogate time as a tool to explore how shifts in spatial conditions over time can create a unique architectural experience. 


\subsubsection{TIME \& SPACE}

Bernard Tschumi's design manifesto is built on the notion that "Architecture is not simply about space and form, but also about event, action, and what happens in space" (The Manhattan Transcripts).

The importance of Tschumi's transcripts to this thesis lies in the definition of 'transcripts', ie. a written or printed version of material originally presented in another medium. Tschumi's Manhattan Transcripts are "neither real projects nor mere fantasies. They propose to transcribe an architectural interpretation of reality" (The Manhattan Transcripts 7).

Tschumi introduced notations in his transcripts as a device to relate events of human physicality with architectural space and form; the drawings and notations of movement in space (Fig. 4 and Fig. 5) become built as pieces of architecture. It is literally taking a diagram and transcribing it into an inhabitation form-a literal meaning to transcript in architecture and a manifesto for the assimilation of architecture into life.

While Tschumi's work also deals with other social, political and cultural issues, the discussion made in this section only covers what is relevant to the framework of this design investigation.

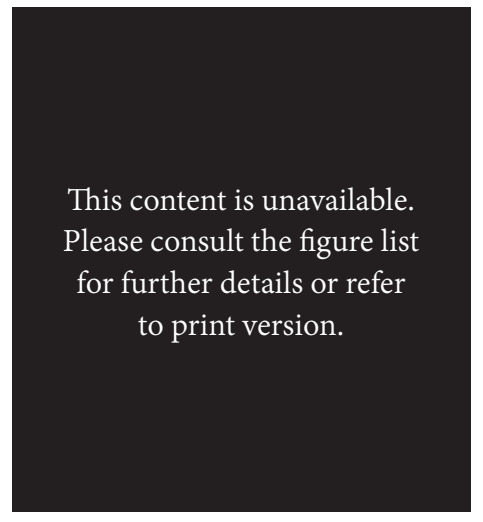

Fig. 4. Tschumi's Notation Diagram "The Fall"

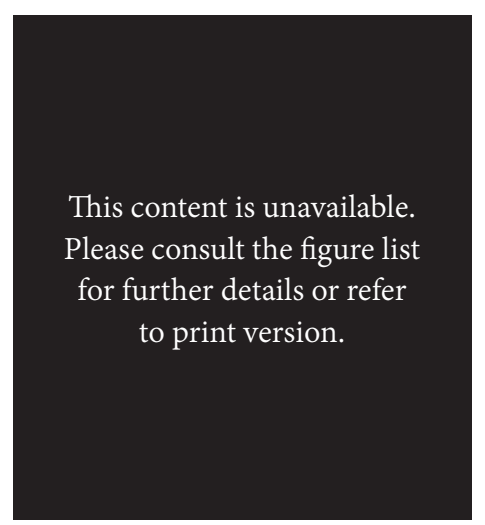

Fig. 5. Tschumi's Conceptual Notation Diagram of Parc de la Villette 
Tschumi's method of diagramming movement is explored as both an abstraction device in thisthesis to conceive formal outcomes of the artefacts as well as an analysis tool throughout the design stages. Tschumi's la Villette is an organised system of forms conceived through his diagramming method of events occurring through time. The design investigation series undertaken in this research employ this method in a similar manner where the forms of the artefacts are conveying the actions and implying the dynamic movement reflective of a selected Eastern superstition. The implied movement is then advanced further in preliminary design stage 2, where the element of time plays a part in the transformation of spatial conditions.

Fig. 6 is a thesis conceptual sketch diagram applying Tschumi's notion style within a grid layout to draw dynamic implications and relationships of the 9 design interventions to explore how the spaces may transform over time.

In the thesis investigation, there is a constant transcribing of this movement bound with event and time through the multiple stages of design.

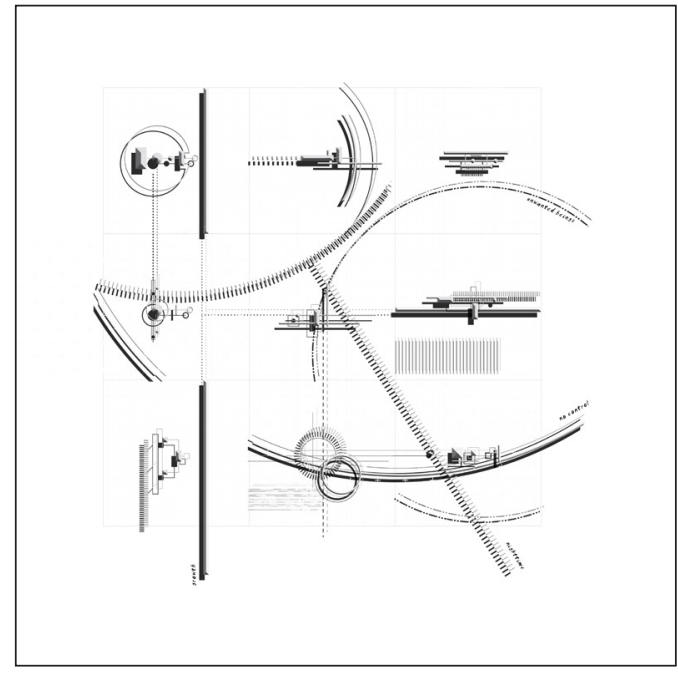

Fig. 6. Conceptual notation diagram drawing relationships and links

Image by Author 


\section{Progress \& Journey}

RO 2: To explore how narrative architecture can activate allegory within the experiential as a tool for reinterpreting the notions of 'traditional' architectural elements through their shift in spatial conditions over time.

The second principal trait of allegory extracted from Haralambidou's writing is the notion of progress-a sequence of events understood as a journey, as proposed and developed by Angus Fletcher in Allegory: The Theory of a Symbolic Mode. This section considers Progress and Journey in relation to Narrative, Timebased Event: Film and Animation, Chronotope, and Removing Authorship. It critically reflects on Progress and Journey in the allegorical architectural project in relation to theorists Jerome Bruner, Cathy Ganoe and Anthony Vidler.

Haralambidou discusses Walter Benjamin's analysis of allegory and how he casts it as primarily an experience-an experience of the world not as permanent, but temporary, fragmentary and enigmatic (226). This section focuses on the formation of temporal spaces that are experienced through the notion of the journey where time is a fundamental element in the construction of an architectural narrative.

The second stage in this preliminary design investigation series introduces time as a factor that shifts the spatial conditions of the formal outcomes conceived through preliminary design stage one. Time is an important aspect of how we experience and understand architecture, but time is rarely used to explore and question spatial and experiential qualities and how they may change over the course of time as new events unfold.

Progress and journey are explored in this thesis through animated schemes where events unfold over the course of time, causing changes in the formal outcomes and shifting the spatial conditions. The notion of progress, journey and time are explored in the Literature and Project Review chapter primarily as experience using fictional narrative theory as a framework that informs and assesses how the experiences are framed.

The discussion will be based on a narrative theory established by Jerome Bruner, winner of the 1987 Balzan Prize and senior research fellow at New York University, who examined how the structure of narrative method organises human perception of understanding in fictional literature. Cathy Ganoe, theorist, professor and researcher in the field of Interior Design at Central Michigan University, analyses and applies Bruner's narrative methods through spatial and experiential senses in interior architecture. 


\subsubsection{NARRATIVE}

Narrative theory acts as a framework in this design investigation that constructs and synthesises the symbolic artefacts to begin to transition into a time-based scheme. Narrative theory can actively participate in the construction of events unfolding over time-as the notion of animated schemes unfolding are very much similar to the construction of fictional narratives. According to Bruner:

The central concern is not how narrative as text is constructed, but rather how it operates as an instrument of mind in the construction of reality (5-6).
Jerome Bruner, in his paper "The Narrative Construction of Reality", extracts ten fundamental features that are the foundation of the construction of a fictional narrative in literature. These ten features will be used in the thesis investigation as the interrogation tools and the theoretical structure to dissect as well as inform the decision-making process of the design exploration series, to add a depth to understanding how space is psychologically inhabited by the individual. Although Bruner's article focuses on narrative as seen in literary fiction, this thesis draws upon his ideas through theories proposed by Ganoe-who reinterprets Bruner's ten components in the context of interior designto argue that narrative as a method of storytelling can be applied in the field of architecture as well. 
Design Criteria and Correlating Components of Narrative

\begin{tabular}{ll}
\hline Design Criteria (Ganoe) & Narrative Content (Bruner) \\
\hline Order & Diachronicity \\
Experience & Particularity \\
State of Mind & Intentional State Entailment \\
Meaning & Hermeneutic Composability \\
Innovation & Canonicity and Breach \\
Transformation & Referentiality \\
Universality & Genericness \\
Multiplicity & Normativeness \\
Subjectivity & Context Sensitivity/Negotiability \\
Convergence & Narrative Accrual \\
\hline
\end{tabular}

Table 2. Narrative Criteria (taken from Ganoe, 3.)
Ganoe writes:

The characteristics of narrative that help to organize the complex world of people, entities, and events through the language of stories provide a flexible framework for understanding and expanding the meanings of design (3).

Ganoe discusses opportunities for personal architectural narrative in the construct of space and how it can be used to shape human experience of space. She examines the role of semiotics and phenomenology in the inhabitation of space and uses Jerome Bruner's components of narrative as a structure to tie the concepts together and develop criteria for constructing architectural design narrative theory.

This section briefly discusses and extracts ideas from Bruner's ten components as applied in the thesis's preliminary design explorations. The main focus will then progress to how some of the components feed into the notion of time and agency, as two main elements that are vital to the construction of narrative in relation to the Research Questions posed in this thesis. 
Bruner posits ten features that he argues are the foundation of the construction of fictional narrative in literature. They are as listed:

\section{Narrative Diachronicity}

Narrative Diachronicity is the way events in a narrative are understood by their relation over time (Bruner 6). Time is a fundamental element in the construction of a narrative. It determines how a story unfolds and how the narrative is understood through the unfolding of events over time. According to Bruner:

The time involved, moreover, as Paul Ricoeur has noted, is 'human time' rather than abstract or 'clock' time. It is time whose significance is given by the meaning assigned to events within its compass (6).

Ganoe applies this concept within the context of an architectural environment as the order of dynamic experience of space over time and through movement, i.e. how understanding and perception of space changes over time (5). Diachronicity is explored in this thesis through the use of time-based events conceived as animations within a digital field.

\section{Particularity}

Particularity refers to the unique and particular attributes that bring interest to the narrative (Bruner 6-7). According to Bruner:

Narratives take as their ostensive reference particular happenings. But this is, as it were, their vehicle rather than their destination (6).

Ganoe's reinterpretation of particularity comes down to the unique experience of the participant and how space can be uniquely interpreted by various individuals to create a meaningful encounter (6). Particularity is explored in this thesis firstly through the unique attributes of Burmese superstitions as provocateurs to achieve unique unconventional morphologies, in Bruner's words, as a "vehicle rather than [the] destination" (Bruner 6). Particularly is next explored through the unique experience of the individual, achieved through the ability of the virtual gaming environment, to offer a new and personalised experience in every simulation. 
3.

Intentional state entailment is the way a narrative is constructed to give readers the freedom of choice in interpretation of the text, and the author does not dictate how it should be interpreted (Bruner 7). In the context of space, Ganoe establishes that the intentional state entailment can be considered as a state of mind - the consideration of creating space that can be experienced by individuals of different backgrounds, intentions and psychological traits (6). This could include the element of agency introduced to invite flexibility in relation to how the individual intends to use and inhabit the space using their own free will. This thesis explores intentional state entailment through the element of agency introduced in the computer gaming stage where the navigation within space is up to the player to decide, and this determines their own experience of the space.

\section{Hermeneutic Composability}

As previously explored in Barthes's semantic codes, the concept of hermeneutic composability is the interpretation of meaning not necessarily being the same as that of the intended meaning. This leaves interpretation to be constructed by the participant to see it as they wish; there is simply no one answer or truth (Bruner 7-11).
According to Bruner:

there is no unique solution to the task of determining the meaning for this expression (7).

In relation to Bruner's proposition about hermeneutic composability, Ganoe interrogates how forms can be assembled to construct complex yet understandable meanings and how different people associate the same forms with similar meanings (6-7). This concept is explored in this thesis in the construct of meaning in symbolic forms-the signifier and the signified.

\section{Canonicity and Breach}

Telling the story in innovative and nonconventional ways is how Bruner establishes the breach of canonicity (11-12). According to Bruner:

For to be worth telling, a tale must be about how an implicit canonical script has been breached, violated, or deviated (11).

Ganoe relates this concept to the context of the environment as the innovation in using form and space unconventionally to convey original meaning (7). This thesis draws from Burmese superstitions as the provocateurs for the genesis of unique forms that evoke a sense of supernatural mystery. 
6.

\section{Referentiality}

Referentiality refers to when the narrative creates a reality by referring to a collection of verisimilitudes (the appearance of truth) (Bruner 13). According to Bruner:

Narrative 'truth' is judged by its verisimilitude rather than its verifiability (13).

Alternate realities are capable of heightening our experience, and a space of transformation that can act as "a place where inner and outer realities may merge" is how Ganoe describes the notion of referentiality (8). When this alternate reality is capable of directing and altering the participant's heightened sense of self through verisimilitudes, this can impact how the individual makes references to reflect upon the space. This thesis explores spatial concepts through the construct of a virtual dream-like autonomous world that is undefined by real-world laws and logic, but its concepts draw from real-world truths and ideas.

\section{Genericness}

The invention of a 'way of telling' the story in a language that can be understood by all cultures is how Bruner establishes the notion of genericness (14-15). He makes the claim that a narrative, with the ability to connect with people of all cultures, can have a powerful influence over the way it is able to shape our modes of thought (15). Ganoe refers to this as the notion of universality - space as a common genre that can be understood by all, which thus makes it powerful in shaping our broader sense of human experiences of inhabiting space where reflection of the world and self can be awakened (9). The thesis aims to find possible solutions for architecture that may be rooted in cultural meaning, but are not defined by cultural ideals and rules, so they can be interpreted and understood by unique individuals. This attempts to look at the overarching issues in today's advancements of globalisation and the loss of unique cultural values that have been affected by it. Architecture, as a discipline that invites interpretation from all cultures, becomes a powerful tool in conveying and unveiling these concepts.

\section{Normativeness}

Normativeness refers to the establishment of norms within the construct of a narrative that is in a constant state of flux (Bruner 16). The stories are always changing by the reconfiguration of personal interactions with the world. Ganoe interprets this as the multiplicity of the experience of space and how it can never be truly experienced or interpreted the same way by any two people (9). The established norms that are the construct of space can never truly be fixed. 
According to Bruner:

These norms are located within the changing narrative of the individual in relationship with theirphysical, socialandpsychologicalworld(9).

This notion is explored in this thesis through the element of agency provided by the computer gaming environment that introduces endless outcomes of multiple experiences that can differ from one player to another. In a richly complex and creative virtual world, no two players will experience the same space in the same way due to each individual's own unique relationship with their physical, social and psychological world.

\section{Context Sensitivity / Negotiability}

Context sensitivity and negotiability is the understanding that narrative relating to a certain culture holds its own truths while inviting other cultures to share these ideas. It is a platform for negotiating meaning where "'meanings' are sought for, not meanings imposed" (Bruner 17). According to Bruner:

It is this very context sensitivity that makes narrative discourse in everyday life such a viable instrument for cultural negotiation. You tell your version, I tell mine, and we rarely need legal confrontation to settle the difference (17).
Ganoe translates this as the notion of subjectivity in the spatial realm where experience is purely subjective and one's experience will differ from another, but there is no need to settle the difference (10). This relates again to the element of agency, allowing the participants within the gaming platform to construct their own pathways and meanings to create their own subjective experiences. This also addresses the cultural factor where meanings extracted are sought and not imposedthe idea that there are no culturally imposed ideals but rather interpretation is invited instead.

\section{Narrative Accrual}

Narrative accrual is the notion of a collective representation-how the story is understood as a collection of elements becoming a whole (Bruner 19). Ganoe refers to this as convergence-the embracing of diverse methods in the goals and outcomes (12). This collective representation is explored in two ways in this thesis: firstly, in the diversity of interrogation methods-drawings, physical models, animations and the gaming environment-and secondly, in the uniting of 9 interventions based on Burmese superstitions to coexist as a collective narrative. 
Narrative Constructs Bruner's Definition for Literary Fiction
Ganoe's Interpretation for Architectural Spatial Context

ORDER - how the same space can appear or be experienced differently over a period of time.
Author's Application for the Thesis Investigation

Introducing time-based animated events to construct an architectural narrative where the architecture is experienced over time.

$\begin{array}{ll}\text { 2. Particularity } & \text { Unique and particular } \\ \text { attributes to bring interest } \\ \text { to the narrative. }\end{array}$

\section{EXPERIENCE-how the} experience of space can be unique to the individual.
The particularity of the artefacts and the uniqueness of its constituent parts (kinetic + stationary).

\section{Intentional State Entailment}

Allowing for agency, the presumption of choice. To give participants the freedom of choice in interpreting the text, the author does not dictate how it should be interpreted.

\author{
STATE OF MIND—how \\ various individuals of diverse \\ backgrounds respond differently \\ to the same space.
}

The transitioning into a virtual gaming environment where the user becomes a player free to navigate within the constructed spatial realm.

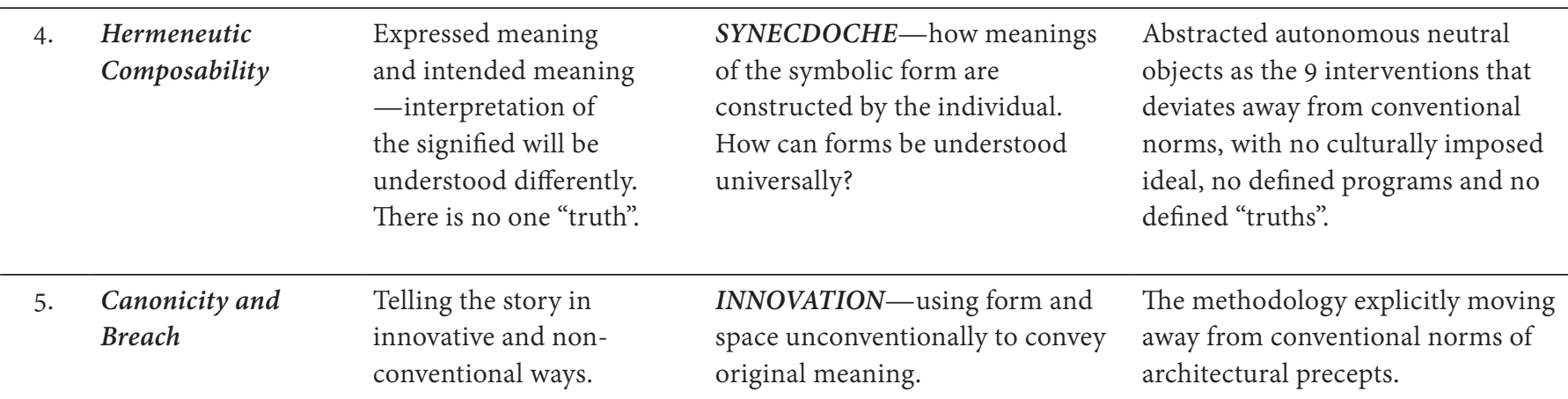

Table 3. Narrative Constructs Criteria Comparison 


\begin{tabular}{|c|c|c|c|c|}
\hline 6. & Referentiality & $\begin{array}{l}\text { Narrative 'creates' a } \\
\text { reality, rather than } \\
\text { simply referring to a } \\
\text { reality by collecting } \\
\text { verisimilitudes (the } \\
\text { appearance of truth). }\end{array}$ & $\begin{array}{l}\text { TRANSFORMATION_to enable } \\
\text { space to direct the participant's } \\
\text { sense of self into an alternate } \\
\text { reality to heighten experience } \\
\text { while creating references to truths. }\end{array}$ & $\begin{array}{l}\text { The construct of a virtual dream-like } \\
\text { autonomous world defined by its own } \\
\text { laws and logic, undetermined and } \\
\text { unbound by real-world constraints } \\
\text { while still being based on concepts } \\
\text { of real-world truths and ideas. }\end{array}$ \\
\hline 7. & Genericness & $\begin{array}{l}\text { The invention of 'a way } \\
\text { of telling' the story in } \\
\text { a language that can be } \\
\text { understood by all cultures. } \\
\text { As such, it can have a } \\
\text { powerful influence in } \\
\text { shaping our modes of } \\
\text { thought. }\end{array}$ & $\begin{array}{l}\text { UNIVERSALITY - the broader } \\
\text { human phenomenon of inhabiting } \\
\text { space and allowing for a space } \\
\text { where self-reflection can be } \\
\text { awakened. }\end{array}$ & $\begin{array}{l}\text { The removal of culturally imposed } \\
\text { ideals and the denotations of } \\
\text { concepts to arrive at abstracted } \\
\text { architecture that can be understood } \\
\text { by all. }\end{array}$ \\
\hline 8. & Normativeness & $\begin{array}{l}\text { The establishment of a } \\
\text { norm, so that it can later } \\
\text { be "breached". }\end{array}$ & $\begin{array}{l}\text { MULTIPLICITY - space is never } \\
\text { experienced the same way by any } \\
\text { two people and cannot be entirely } \\
\text { predicted or controlled. }\end{array}$ & $\begin{array}{l}\text { Agency - introducing endless } \\
\text { outcomes and possibilities of spatial } \\
\text { experience that differ from one } \\
\text { player to another. }\end{array}$ \\
\hline 9. & $\begin{array}{l}\text { Context Sensitivity / } \\
\text { Negotiability }\end{array}$ & $\begin{array}{l}\text { Ideals and meanings } \\
\text { are not imposed but } \\
\text { are rather negotiated } \\
\text { through one's own } \\
\text { cultural processes. }\end{array}$ & $\begin{array}{l}\text { SUBJECTIVITY - space is a } \\
\text { strictly subjective experience. }\end{array}$ & $\begin{array}{l}\text { The element of agency to allow } \\
\text { users of all ages and cultures to } \\
\text { experience the space through their } \\
\text { own constructed pathways, to } \\
\text { construct their own meaning in } \\
\text { interpretation of the space. }\end{array}$ \\
\hline 10. & Narrative Accrual & $\begin{array}{l}\text { Making the parts become } \\
\text { a whole: collective } \\
\text { representation. }\end{array}$ & $\begin{array}{l}\text { CONVERGENCE-embracing } \\
\text { diverse methods in the goals and } \\
\text { outcomes. }\end{array}$ & $\begin{array}{l}\text { The uniting of } 9 \text { interventions } \\
\text { to co-exist within one realm to } \\
\text { introduce new configurations, } \\
\text { relationships and dialogues. The } \\
\text { merging of mediums to shift the } \\
\text { concept from a physical realm and } \\
\text { into the digital. }\end{array}$ \\
\hline
\end{tabular}




\subsubsection{TIME-BASED EVENT: FILM AND ANIMATION}

"In the same way that architecture articulates space, it also manipulates time."

-Juhani Pallasmaa 20

The concept of time is explored through Bruner's notion of diachronicity, and this section focuses on how diachronicity can be explored through the ability of film, as an animation medium, to convey time-based events. Bruner's concept of diachronicity explored in this chapter involves the understanding of events as they unfold over time. Time is a fundamental aspect of how we experience architecture, but time is rarely ever explored in architecture in relation to spatial and experiential qualities and how they may change over a course of time as new events unfold or as the viewers themselves changes their course of action. This thesis aims to examine the unfolding of events over time through digital animation as a design medium, arguing that it offers an avenue to producing unexpected spatial outcomes through the simulation and visualisation of changes in spatial conditions over time. It allows us to question architectural spatial conditions through a mode that static methods of inquiry do not readily allow.

The thesis's preliminary design exploration series challenge the very ideals of architecture and attempt to remove real-world constraints of being static and fixed, to instead explore how concepts being conveyed can only be achieved through the dynamic shift in the morphologies evidenced by the animation medium. It aims to interrogate architecture and space within the construct of a virtual world where the preconceived notion of a frozen and idealised conception of architecture is immediately removed, and instead space becomes an experience that has its own duration actualised by events unfolding over time. 
Anthony Vidler, Professor of Architecture at the Copper Union, discusses in his article "The Explosion of Space: Architecture and the Filmic Imaginary" that the fascination with film in architecture is the ability to construct its own architecture, its own reality (46). He discusses art historian Jacques Élie Faure's concept of 'cineplastic': the plastic nature of cinema as it represents architecture in movement when it is traditionally understood as being in constant accord. Faure claims therefore that time is an instrument of space. The ability of cinema, to be able to express forms at rest and in movement, gives rise to "unknown plastic pleasures" to be discovered within the creation of new architectural and imaginary space "within the walls of the brain" (Vidler, The Explosion of Space, 46). According to Faure:

"the cinema incorporates time to space. Better, time, through this, really becomes a dimension of space."

-Faure 268 


\subsubsection{CHRONOTOPE}

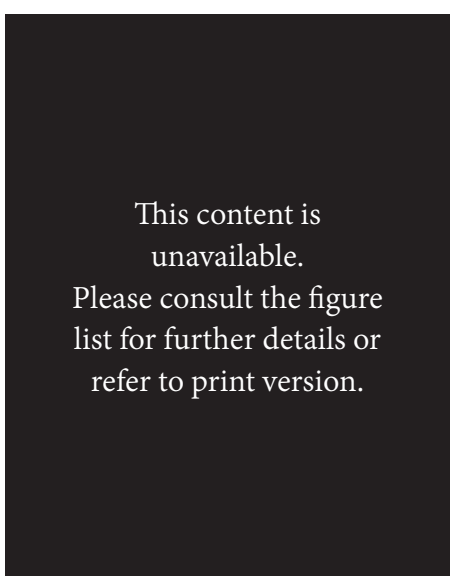

Fig. 7. Hejduk's Masques: PainterStudio A

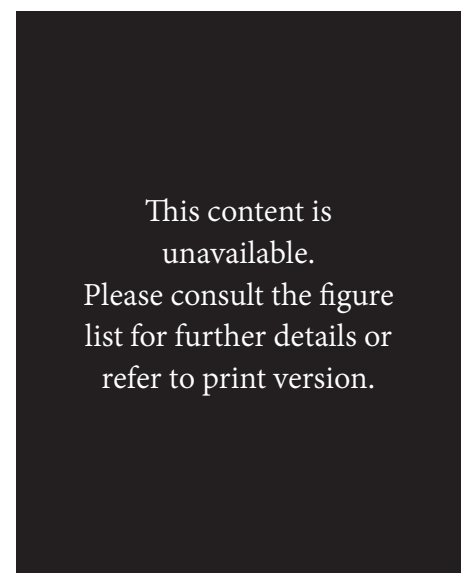

Fig. 8. Hejduk's Masques: Musician-Studio B

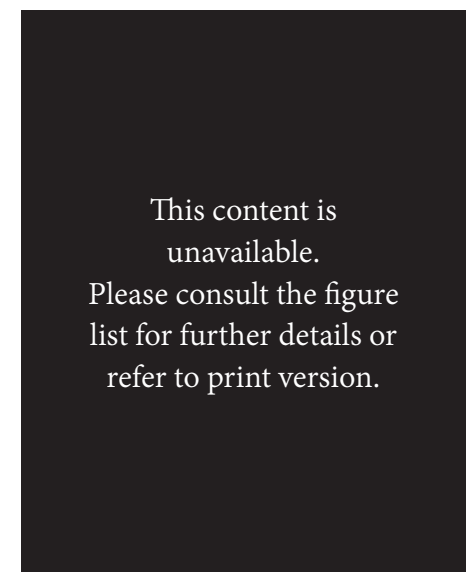

Fig. 9. Hejduk's Masques: Security-Structure

\section{Case Study: John Hejduk's Masques}

American architect and educator John Hejduk developed "masques" that explore the notion of artefacts that exist within their own reality, in a dreamlike theatrical/architectural game bound by its own structure of time and space. Architectural historian K. Michael Hays writes about Hejduk's work as a type of 'chronotope': "the coordination of a system of time and space, a form-giving ideology" (10). The concept of chronotope derives from language and literature proposed by Mikhail Bakhtin, a Russian literary scholar.
Hejduk's masques are designed as autonomous architectural elements that are able to move from place to place in a ritualised geographic performance, and their morphological properties are often derived from concepts of time. Although the masques depict ideas of time and space, the forms are very much out of time and place. "Like a poem, they have a logic of their own," writes Wim van den Bergh in Hedjuk's The Lancaster/Hanover Masque; "they ... display a 'mysterious sense' which lends them an intense evocative power. They are operational scenarios within an architecture that merely simulates - and does not in itself containan 'actual sense"' (Bergh 83-84). Bergh writes that 
Hejduk's masques are structured like free scenario matrices: "synthesis-machines" comprised of an organised system of geometries and symbolism, and they are without identity. To truly serve the many cities that the masques visit, to be able to identify with each unique city, the masque empties itself of any architectural and cultural traditions.

Among Hedjuk's numerous architectonic figures relating to concepts of time, the Clock/Collapse of Time (Fig. 10), as seen in his work Vladivostok, is an exceptional example of the chronotope that takes the form of time as well as moves through spatial time; the clock tower is mounted on wheels so that it can be moved from place to place by the townspeople, and it also is comprised of a wooden element that moves through the angles of a clock (Hejduk, Vladivostok 72).

Hedjuk's moving masques synthesise the notion of: timeless autonomous neutral objects; time through theatrical movement shifting the spatial conditions through form and configuration; as well as situating themselves within a game-like reality. The masques move from one geographic location to another, forming new relationships and dialogues with the cities that they encounter. This concept is challenged and explored within the preliminary design series of this project through the use of animation tools, unravelling new conceptual shifts in the morphologies of the artefacts.

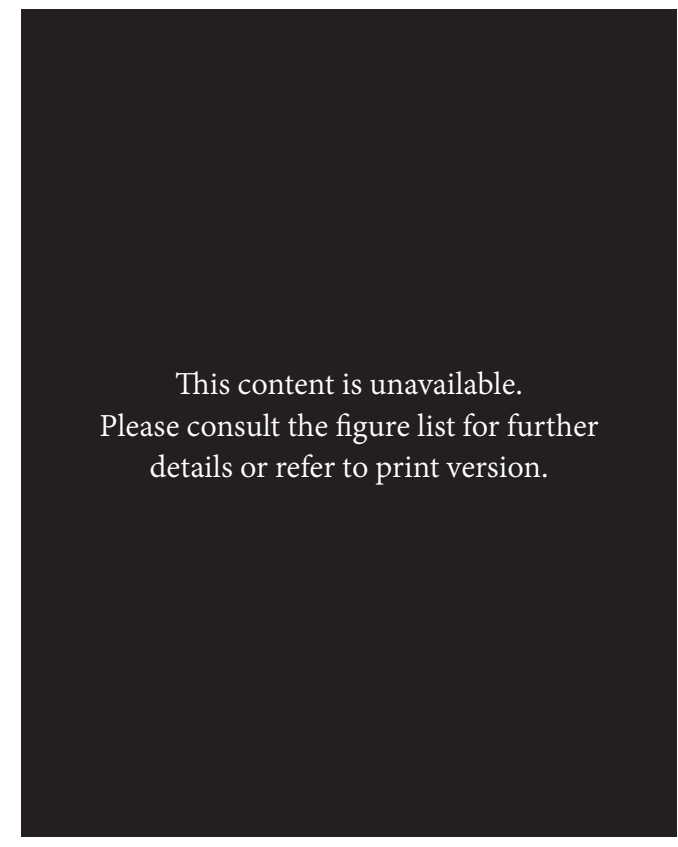

Fig. 10. Hejduk's Masques: Clock/Collapse of Time 
The masques exist as a collective whole in the works of Hejduk (Fig. 12). The experience of navigating through the masques is said to be game-like, where the participant becomes absorbed in the architectural imaginative reality constructed by the objects. According to Bergh:

We have to become part of this labyrinthine choreography whose only goal is the feast of the game itself. We can proceed without worrying about what direction to take, there is no need to arrive anywhere: we are bound up in the Masques and drift along with them. This is a game of imagistic awakening, characterised by a simultaneous engagement and distance... we have to join the game... but we do not know in advance what the encounter will entail: the poetic appears only after the encounter, after the playing of the game.
Hejduk's masques reflect an architectural game being inhabited by players to construct their own realism and truths, no longer bound by the authorship of the creator: "no longer depend[ing] on the violation of the architect, the artificer" (Bergh 85).

The "inhabitants" (players) of the Lancaster/ Hanover Masque "live/dwell" in an architectural matrix which they themselves have to animate in order to evoke the idea of enclosure and centrality in the simulation of their "dwelling". In otherwords, "dwelling" is no longer represented by the architecture, but has to be produced by the "inhabitants" (players).

-Bergh 84

-Bergh 87

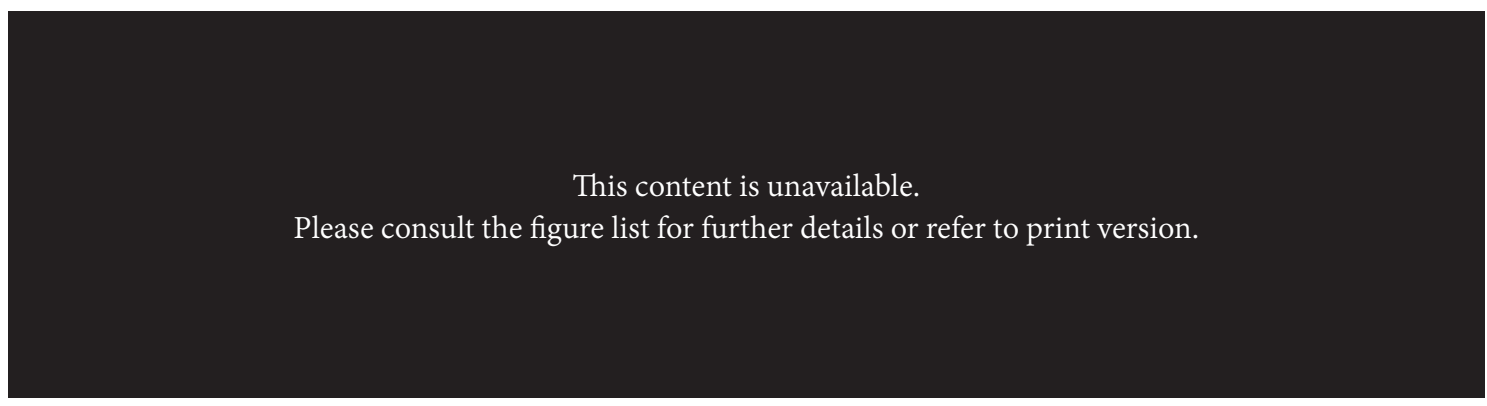

Fig. 11. Hejduk's Masques 
This content is unavailable.

Please consult the figure list for further details or refer to print version.

This content is unavailable.

Please consult the figure list for further details or refer to print version.

Fig. 12. Hejduk's Victims Drawing Compilations of Masques coexisting as a collective whole 


\subsubsection{REMOVING AUTHORSHIP}

In Vidler's essay, "The Explosion of Space: Architecture and the Filmic Imaginary", he discusses the two categories of the spatial eye in film, as established by a Soviet filmmaker trained as an architect, Sergei Eisenstein: the cinematicwhich is the eye of the viewer being directed by the camera-and the architectural-which is more about the movement of the viewer within the space to direct their own perception (56). Both concepts concern the perception of sequence and order in space to form an experience, but the latter introduces the element of agency, allowing the viewer to construct their own spatial experience. Film and animation offer the ability to narrate events unfolding over time; but when the dictatorship over selected frames is removed, the architectural spatial eye can be achieved, unlocking the element of agency, which will be explored further in this thesis through the medium of the game environment. 


\title{
2.3 EXPERIENTIAL ARCHITECTURE:
}

\section{Invitation for Interpretation}

\begin{abstract}
RO 3: To explore how Digital Gaming Environments can be actively engaged in the architectural design methods and processes to enable agency to actively participate in the experiential narrative outcomes.
\end{abstract}

\subsubsection{AGENCY}

The third principal trait of allegory as mentioned in Haralambidou's paper is the ability of the work to invite interpretation. This section considers Invitation for Interpretation in relation to Agency, Individual's Experience as a Narrative, Digital Gaming Environment, and Chronogram. It critically reflects on Invitation for Interpretation in the allegorical architectural project in relation to theorists Jerome Bruner, Cathy Ganoe and Sophia Psarra.

Allegorical architectural works are structured so as to be understood in similar ways as a work of art, where meaning is not able to be grasped directly but through metaphors, and thus these works prompt an open-ended interpretation from the receiver.
According to Haralambidou:

We find an intentional single secondary meaning ... when a work invites an openended interpretation from the receiver, and a concealed meaning to be deciphered through a series of logical steps in an enigma or riddle. Finally, critical interpretation unearths meanings often not intended by the author...

...The work succeeds if it triggers many interpretations, because it is not the artist alone who performs the creative act: the viewers make their own contribution. It is clear, however ... that he [the artist] sees the work presented as a riddle or an enigma not only to the viewer but also to the author. Therefore, the work of art is an allegory in receiving, interpreting, but also in making.

- Haralambidou 230-231 
The importance of the Invitation for Interpretation is in the nature of the ambiguity of the work, the multiplicity of outcomes and answers it offers, and the contribution of both the artist and the receiver to perform the creative act. This concept is introduced into the thesis project as an element of agency within a computer gaming medium. Within the constructed journey of the game, the player is free to navigate through the environment to discover new spatial player relationships from a point of view that would differ from other players' experiences. In the preliminary design phase of the thesis investigation, the merging of nine very distinct architectural artefacts also reveals and unearths new dialogues between each other through their respective animated parts. Even as the architectural designer of the animated sequences, the final outcomes of the unified nine sequences are unexpected by me and create a work of art that is "an allegory in receiving, interpreting, butalso in making" (Haralambidou 231).

The previous section discusses time as a fundamental aspect of how we experience architecture and how the time-based animated scheme attempts to challenge the norms of architectural precepts. This section considers how time is also rarely explored in architecture in relation to how spatial and experiential qualities may change as participants of the spatial experience change their course of action. The notion of Fletcher's progress and journey as primarily an experience is further redefined by introducing agency as an invitation for interpretation through the individual's unique experience within a space.
The third stage of design development in the thesis employs the digital gaming environment to introduce a personalised experience that further challenges the conventional ways that architecture is perceived, with the intention that through this shift in perception, the elements of architecture in question could be further redefined through the diverse perceptions of various participants. 


\subsubsection{THE INDIVIDUAL'S EXPERIENCE AS A NARRATIVE}

An individual's own experience and perception determine their unique interpretation of space. Ganoe argues that our understanding of space is not exclusive; it is fluid and undetermined (4). An individual's interaction with the world, their personal experiences, memory and thought are all contributing factors that place the definition of spatial understanding in a constant state of flux due to the very nature of these personal experiences being variant from one individual to another.

This section discusses the use of narrative theory as an additional framework to introduce a personalised depth to the construction of the individual's unique experience. The characteristic of personal narrative over a course of time is discussed as a method that structures and organises a complex world of architectural artefacts, events and their relationships to the users within the construct of a virtual computer gaming environment.

A narrative framework unravels personal, original and imaginative responses to the individual's experience, which is important to the understanding of human perception of space as it "hold normal referentiality at bay allowing for possibility within impossibility [and] encourage serious appreciation of powerful emotions" (Murray 209).
Ganoe argues that:

By utilizing a structure based on shared language and semantic order, and a content that emphasizes personal perceptions and feelings, the narrative method joins together and augments the semiotics and phenomenological realm of experience as a means of communicating a more complete experience of the individual (4).

Sophia Psarra, author of the book Architecture and Narrative: The Formation of Space and Cultural Meaning, makes a point that narrative requires a narrator to construct and structure a narrative, and a reader to interpret and perceive the narrative, and thus both the narrator and the reader contribute to the construction of the narrative meaning. In a similar way, architecture requires both an architect and a viewer to contribute to the construction of the architectural narrative (2). 
Referring back to Bruner's ten elements of narrative, three features particularly contribute to the construct of agency: the intentional state entailment, normativeness and context sensitivity/ negotiability. Intentional state entailment refers to the state of mind-the freedom of the participant to interpret and extract their own meaning within their own unique experience. Normativeness addresses multiplicity - the nature of outcomes and experiences never being the same for any two people. And context sensitivity/negotiability refers to the subjective experience of space, allowing the differences to be negotiated by participants as a way of telling the story of their own unique journey: "You tell your version, I tell mine" (Bruner 17).

Agency as defined by Bruner is limited to the interpretation of the reader, as the narrative is predetermined by the author. This is the nature and the limitation of a written narrative in literature. By further exploring the reinterpretation of Bruner's text through Ganoe's eyes in the context of the built environment, this thesis aims to challenge and push beyond this limitation to allow for a userconstructed interpretation through the removal of authority, where the journey is undefined and yet to be experienced. The animation medium establishes the notion of the journey as undefined, yet to be experienced, unfolding over time.
Transitioning these concepts into a simulated game environment-a space with its own laws and logic, an autonomous time-space structure-then allows the removal of authority where new narratives and new interpretations can be unravelled, giving the user greater control and a truer sense of agency in the construction of their own truths. According to Ganoe:

\section{... design is capable not only of containing existing} meaning (expressing old ideas) but of creating meaning (expressing new ideas) as well (3).

The notions of subjectivity, multiplicity and state of mind that concern agency can be seen in Italo Calvino's Invisible Cities, a narrative within which the narrator describes the same city in fifty-five different ways. Calvino explains that there are as many versions of a tale as there are listeners; "it is not the voice that commands the story: it is the ear" (135). Invisible Cities evidences concepts of time and place in the description of the city or rather the timelessness of it. The city's identity in present time is moulded by potential futures that at the same time construct multiple possible pasts. This results in a timeless city with which almost any city can identify. The narrative invites means of understanding architecture and reality beyond what it presents at face value - to look beyond the blunt technicalities and instead to imagine potential futures and timeless pasts in the face of our present reality. 


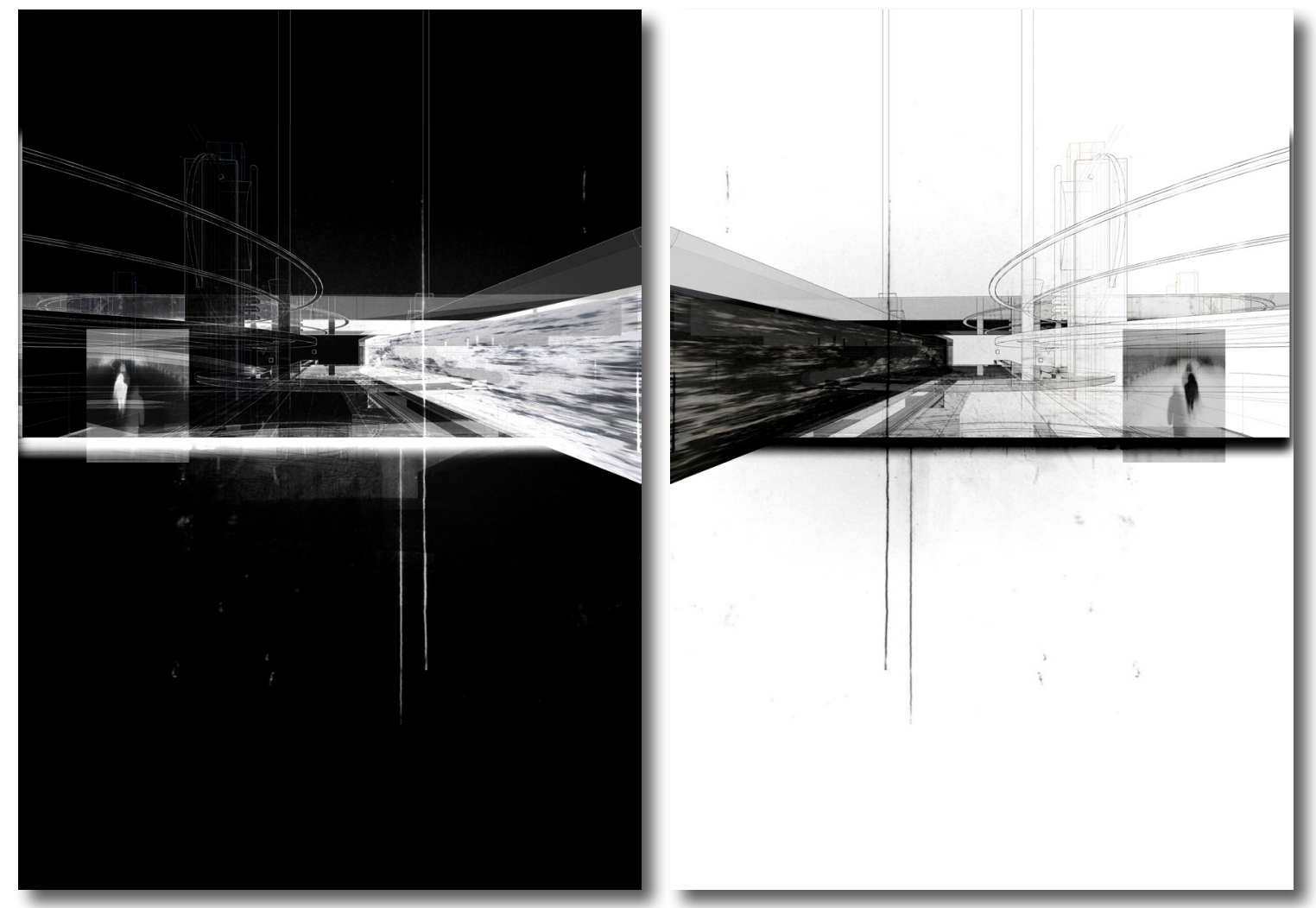

Fig. 13. Conceptual drawings of the multiplicity and subjectivity of the player's perceptive experience Image by Author 


\subsubsection{DIGITAL GAMING ENVIRONMENT}

Narrative is a particular form of representation implementing signs ... bound up with sequence, space and time.

-Paul Cobley (3)

Psarra, in Architecture and Narrative: The Formation of Space and Cultural Meaning, discusses the role of narrative in architecture as not just a form of representation but also as a method and a process. She analyses the definition of these terms as used in David Bordwell's Narration in the Fiction Film where method refers to "a particular way of combining parts to make a whole" and a process as "the activity of selecting, arranging and rendering story material in order to achieve specific time-bound effects on a perceiver" (2). This thesis utilises an experiential method and process of inquiry to challenge the traditional way of perceiving architecture.

\subsubsection{THE CHRONOGRAM}

Nic Clear, professor at the University of Greenwich and renowned for his role in endorsing the techniques of film and animation in architectural education, challenges the modes of architectural representation by introducing into it the element of time. He developed a type of hybrid composite drawing that "combines architectural, graphic and film conventions and attempts to synthesise the various constituent elements by mapping out the formal, narrative, experiential and spatial possibilities of the project alongside the processes of production," which he names the 'chronogram' (78). The term derives from the Greek: 'chrono-' meaning 'time' and '-gram' meaning 'drawing'.

The chronogram is a type of storyboarding technique with an additional depth that maps timebased sequences within a spatial dimension. It is a drawing that attempts to illustrate and capture concepts of space and time in a two-dimensional form. It is a type of storyboarding technique, yet it is so far from the nature of storyboards; "The chronogram is not shot based; it is a spatial drawing. Its role is to determine the spaces of the project" (Clear 78). The chronogram aids the development of an architectural project using moving images and or transcribing the time-based events within a spatial construct by capturing elements of time. 
This content is unavailable.

Please consult the figure list for further details or refer to print version.

Fig. 14. Clear's student's Chronogram: Danai Surasa, Panic Room space-time analysis chronogram.

The chronogram aims to 'map' the space of the film Panic Room. In the drawing, Surasa reconstructs the trajectories of the camera and characters with the spaces of the Panic Room set from the footage, and then maps into that the space of the volumetric footprint of the virtual camera. 
The chronogram is not intended to be as prescriptive as a storyboard; it is a much more fluid drawing type, and should be considered a strategic conceptual aid. Its role is to facilitate the creation of spatial ideas in conjunction with the development of the time-based work.

The chronogram is reinterpreted in this design investigation where the drawing is not a static image but rather the digital game environment itself. The thesis's game environment is similar to how Clear describes the chronogram:

- It is a fluid, strategic conceptual aid that facilitates the creation of spatial ideas through perceptive variability in conjunction with the development of a time-based scheme.

- It combines architectural, graphic and film conventions and attempts to synthesise the various constituent elements by mapping out the formal, narrative, experiential and spatial possibilities of the project alongside the processes of production.

- It attempts to illustrate and capture concepts of space and time except not in a twodimensional form but through an ever-shifting world composed through moving elements.

- It is a spatial drawing.
The interpretation of the digital gaming environment in this thesis as a chronogram provides a similar method and process as established by Psarra and Bordwell: "a particular way of combining parts to make a whole" and "the activity of selecting, arranging and rendering story material in order to achieve specific time-bound effects on a perceiver" (Psarra 2). 
In this chapter, principal theorists and case studies were discussed in relation to the three Research Objectives. The chapter explored Penelope Haralambidou's concept of the "Allegorical Architectural Project" as a framework within which architectural and spatial/experiential concepts can be reinterpreted and redefined.

Haralambidou critically reflects upon three principal traits of allegory--Figurative Geometries, Progress and Journey, and Invitation for Interpretation-in architecture, and these were interrogated as contributors to the theoretical framework on allegory and its application in this thesis investigation. Figurative Geometries enable architectural narratives to engage meaning through allegory; Progress and Journey - understood as a sequence of events - introduce the importance of time, experience and shifting spatial conditions in an allegorical narrative; and Invitation for Interpretation introduces the importance of agency and cultural sensitivity/negotiability within a meaningful allegorical narrative experience.

In the next chapter of the thesis, Preliminary Design, two series of preliminary design experiments will be presented and discussed in relation to the integrated learnings taken from the Literature and Project Review chapter. 
曲 
3. Preliminary Design

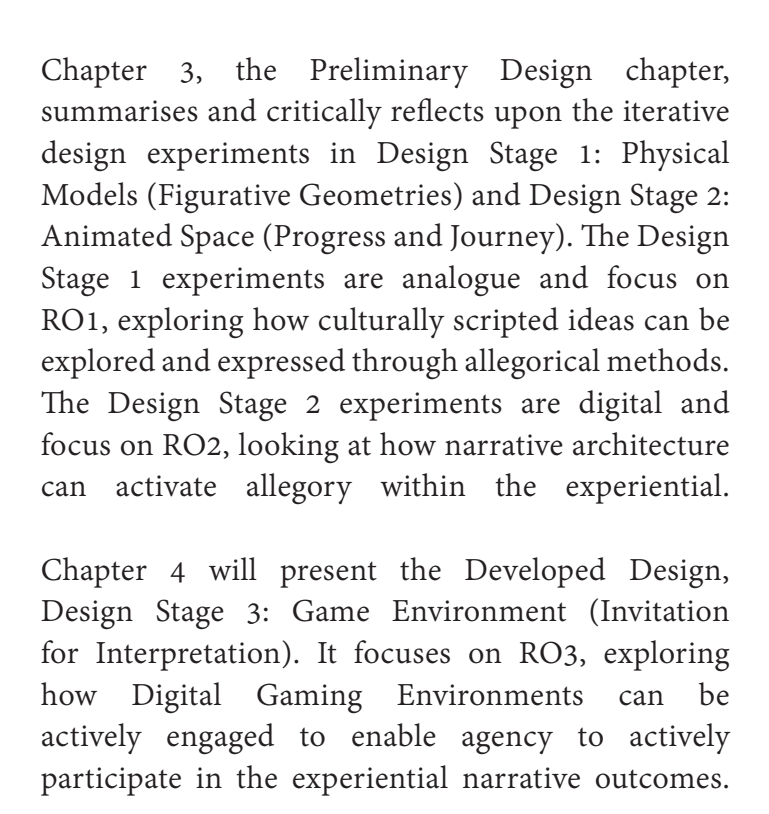


曲 
Abstraction into artefacts

Physical Models

$$
\begin{aligned}
& \text { Singing Old Man } \\
& \text { piece } 1 \text { : aged } \\
& \text { piece } 2 \text { : kinetic | as- } \\
& \text { cension | growth | faces } \\
& \text { piece } 1 \text { at top | }
\end{aligned}
$$$$
\text { 3.1 DESIGN STAGE }
$$

\subsection{DESIGN STAGE}




\section{Physical Models}

\section{FIGURATIVE GEOMETRIES}

RO 1: To explore how culturally scripted ideas can be explored and expressed through allegorical methods in the conception of an evocative architectural framework that deviates from Eurocentric modernist principles and constructs.

If semiotics, beyond being the science of recognized systems of signs, is really to be a science studying all cultural phenomena as if they were systems of signs-on the hypothesis that all cultural phenomena are, in reality, systems of signs, or that culture can be understood as communicationthen one of the fields in which it will undoubtedly find itself most challenged is that of architecture.

—Umberto Eco 173-174 

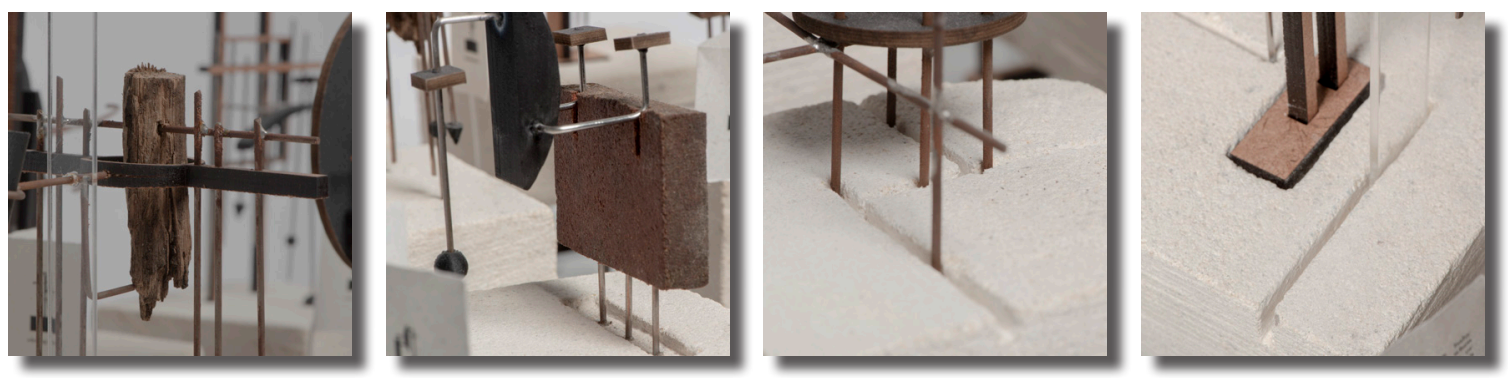

Fig. 15. Close-up photographs of material palette used in the preliminary design models

Design Stage 1 is a set of preliminary design explorations that focuses on developing morphologies of sculptural artefacts through the abstraction of nine selected superstitions drawn from Eastern culture.

The supernatural and mystical nature of superstitions, also having derived from cultural roots, provides an evocative allegorical (as well as narrative) conceptual framework for generating early preliminary design concepts for this design research.

The abstraction process involved using design to extract the fundamental essence of each of nine superstitions. Each physical model attempts to formally express the concepts of each superstition into the morphological properties of an 'architectural artefact'.
The goal was to script the 'artefacts' with symbolic meaning, both in the formal properties as well as materiality of the selected palette. Some of the early physical models convey kinetic concepts, based on action and event, as most of the superstitions involve an act or trigger.

Roland Barthes's theory of the five Semantic Codes are interrogated and interpreted in this formal exploration series to conceive nine artefacts that can be understood as an allegorical 'figurative geometry'.

This design exploration series is a generative exploration using unconventional provocateurs to arrive at evocative outcomes-outcomes that actively avoids adhering to traditional architectural precepts. Their unique attributes offer opportunities rich with symbolic allegorical meaning and potential to develop the series further into innovative outcomes through the next two design stages that follow. 


\section{Artefacts}

The generative nature of the figurative geometry is applied in this thesis to help conceive a set of architectural artefacts to establish an allegorical framework through which architectural spatial concepts can be advanced. In this thesis investigation, an 'architectural artefact' implies that it is only a remnant of a whole, and it suggests belonging to cultural and/or historic origins. The concept of an 'architectural artefact' is applicable to this thesis since the superstitions originate from a strong Eastern lineage, and the abstraction process has fragmented the concept to generate figurative geometriesreducing the signifier into remnants of a whole.

Design Stage 1 begins with 9 selected superstitions that are taken through an allegorical process of abstract mediation to produce 9 artefacts as sculptural physical models. 


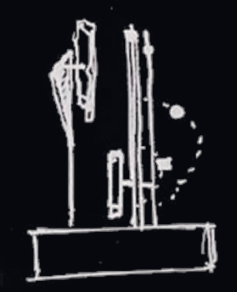

1. singing old man

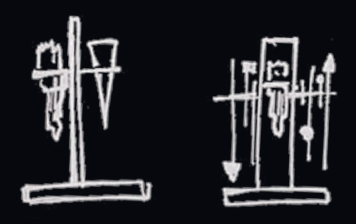

4. Mirror + nightmanes
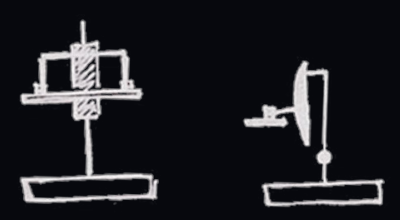

7. elbow

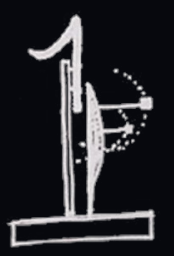

2. Whisting snakes

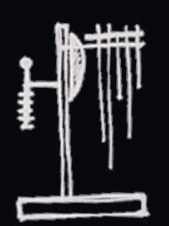

5. clipping nails

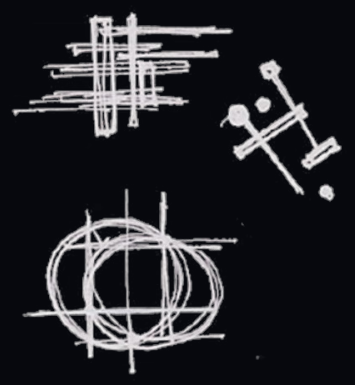

8. Twitching eyes
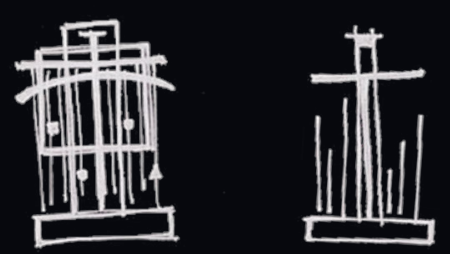

3. umbuelle ghosts

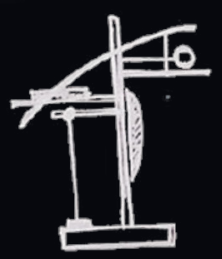

6. baby tooth

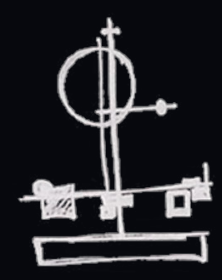

9. Sleep parabsis 

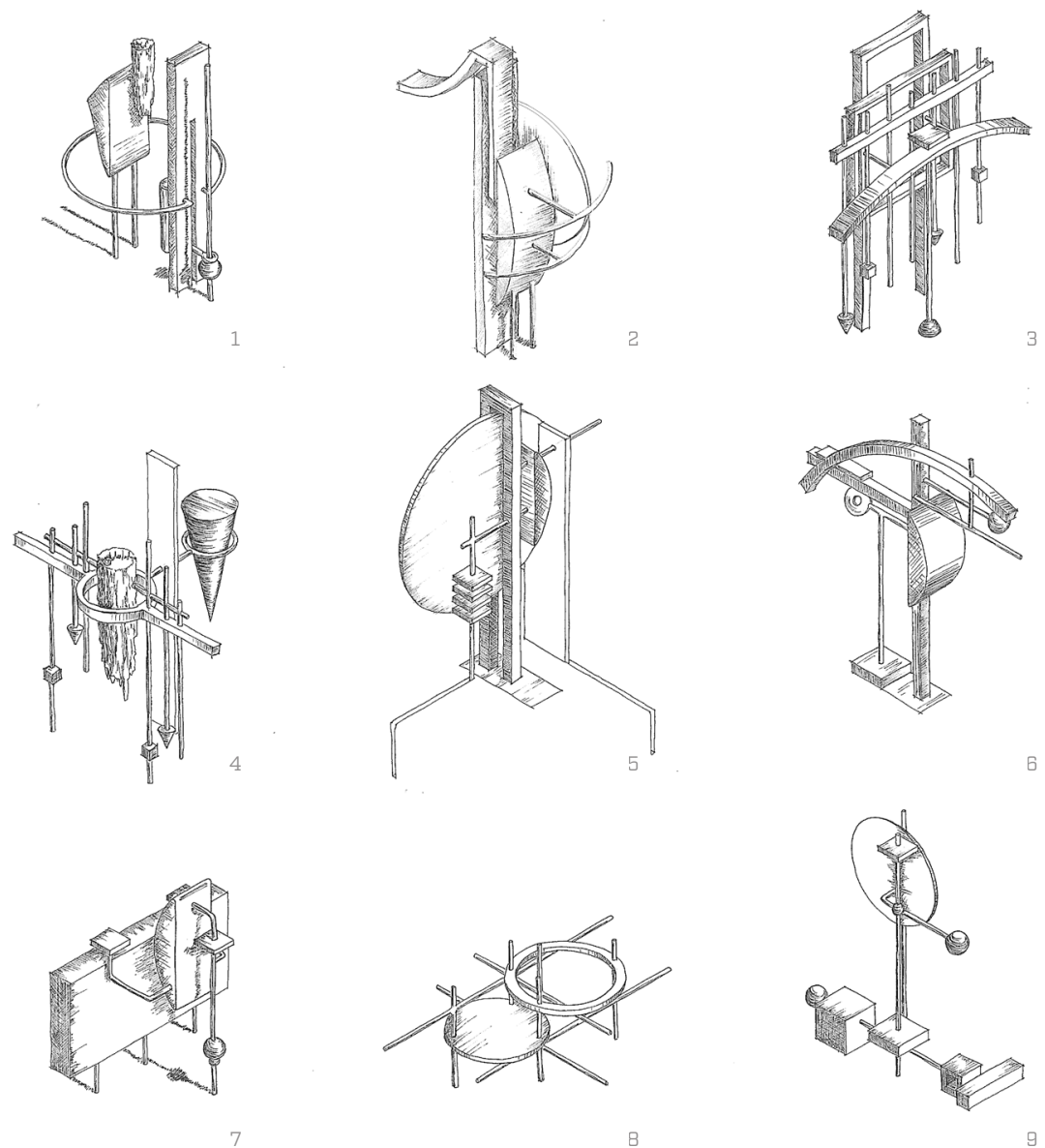

Fig. 17. Sketch of 9 Artefacts 


\begin{tabular}{|c|c|c|c|}
\hline 1. & Singing Old Man & $\begin{array}{l}\text { When singing while eating, one may grow up to } \\
\text { marry an old man. }\end{array}$ & $\begin{array}{l}\text { Growing up to face an } \\
\text { undesired fate. }\end{array}$ \\
\hline 2. & Whistling Snakes & $\begin{array}{l}\text { Whistling at night will invite snakes into one's } \\
\text { home. }\end{array}$ & Invitation of the unpleasant. \\
\hline 3. & Umbrella Ghosts & $\begin{array}{l}\text { Opening umbrellas indoors is an invitation for } \\
\text { ghosts. }\end{array}$ & $\begin{array}{l}\text { Invitation of the unpleasant: } \\
\text { supernatural realm. }\end{array}$ \\
\hline 4. & Mirror Nightmares & $\begin{array}{l}\text { Sleeping with feet facing a mirror will incite bad } \\
\text { dreams. }\end{array}$ & State of the dreamer. \\
\hline 5. & Fingernail Ghosts & Clipping nails at night will provoke ghosts. & $\begin{array}{l}\text { Provoking and invitation of } \\
\text { ghosts. }\end{array}$ \\
\hline 6. & Baby Tooth & $\begin{array}{l}\text { Throw broken baby tooth upwards when it's a } \\
\text { bottom tooth or downwards when it's the top so } \\
\text { that the new teeth will know which way to grow. }\end{array}$ & Scale, balance \& growth. \\
\hline 7. & Elbows of Misfortune & $\begin{array}{l}\text { Don't rest elbows on the dining table, or else one } \\
\text { may end up poor. }\end{array}$ & Consequences \& growth. \\
\hline 8. & Twitching Eye & A twitching eye means someone is missing them. & Separation \& longing. \\
\hline 9. & Sleep Paralysis & $\begin{array}{l}\text { When one experiences sleep paralysis, it is be- } \\
\text { cause a ghost is sitting on them. }\end{array}$ & Paralysis, lack of control. \\
\hline
\end{tabular}


The 9 artefacts were conceived as physical models -9 figurative geometries that can be understood as an 'architectural artefact'. They are scripted with cultural allegorical symbolic meanings. Each piece represents the interpretation of a unique allegorical narrative driven by a selected superstition.

Each of the these early conceptual sketch models will be discussed in further detail. 


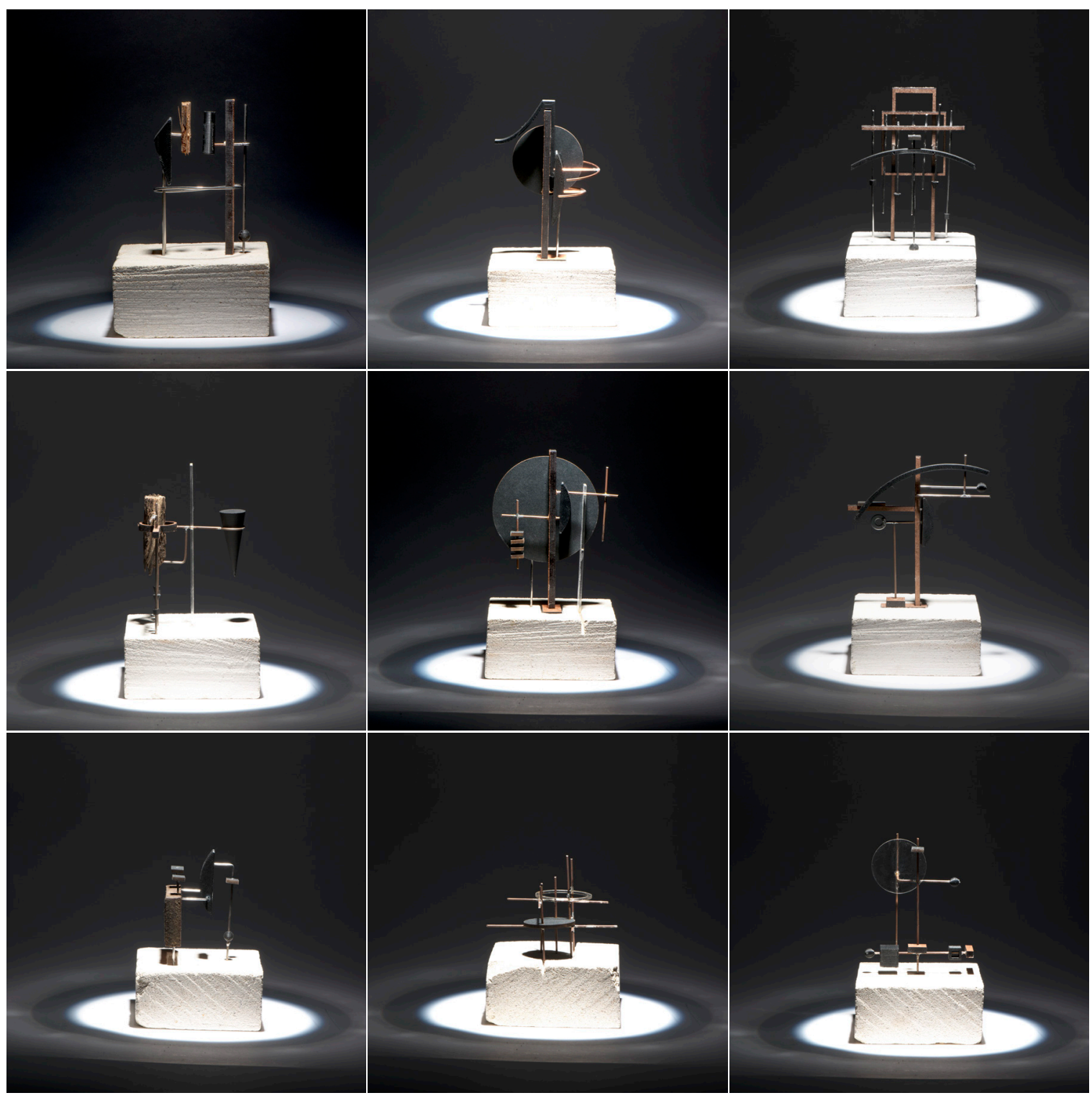

Fig. 18. Photographs of the 9 Artefact Models [Elevational Perspective] 


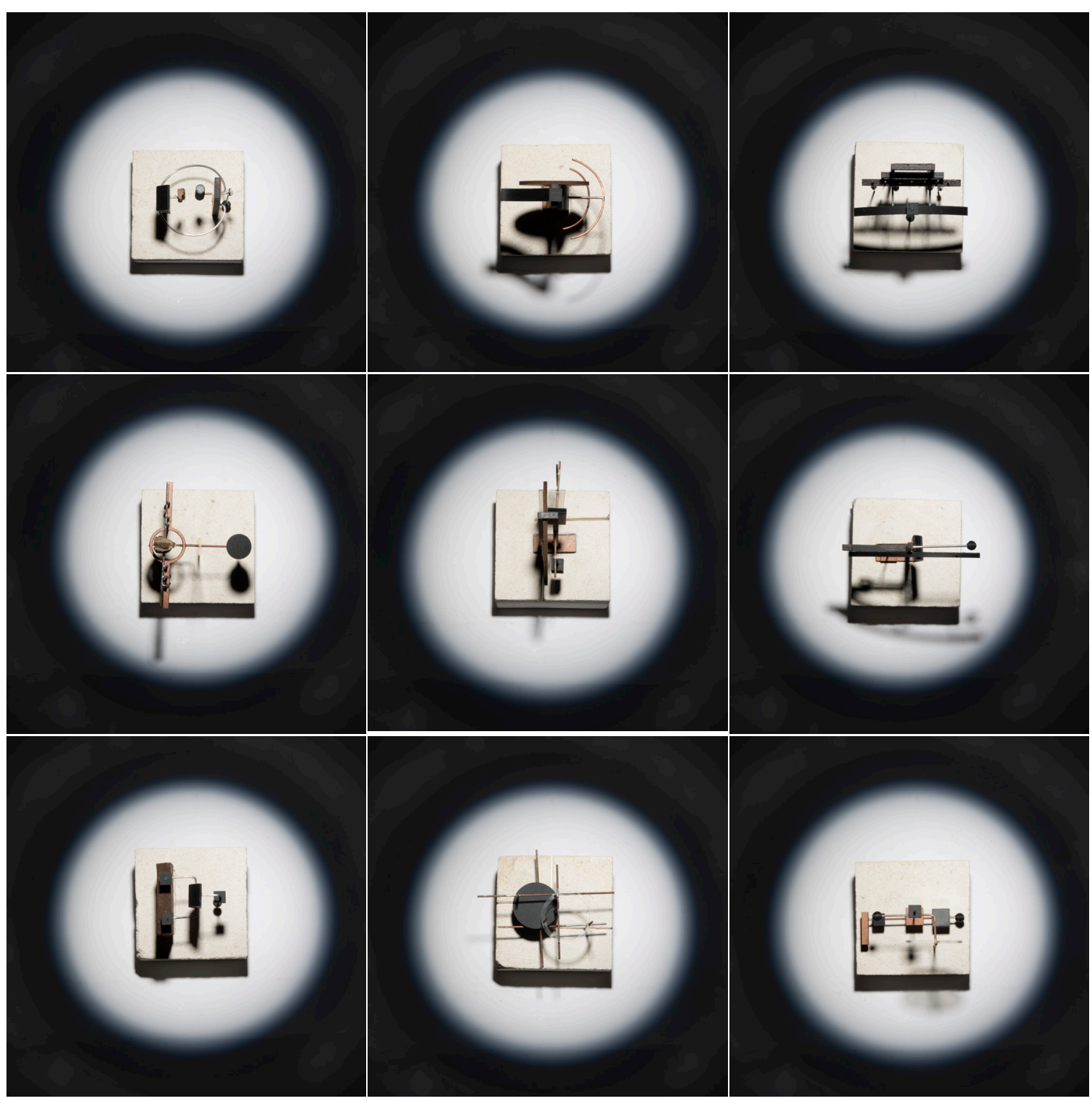

Fig. 19. Photographs of the 9 Artefact Models [Top Perspective] 
The following section of the chapter describes each of the nine selected superstitions and how they were reinterpreted as 'architectural artefacts' in the form of hand sketches and physical concept models. 


\section{ARTEFACT 1}

\section{Singing Old Man}

When singing while eating, one may grow up to marry an old man.

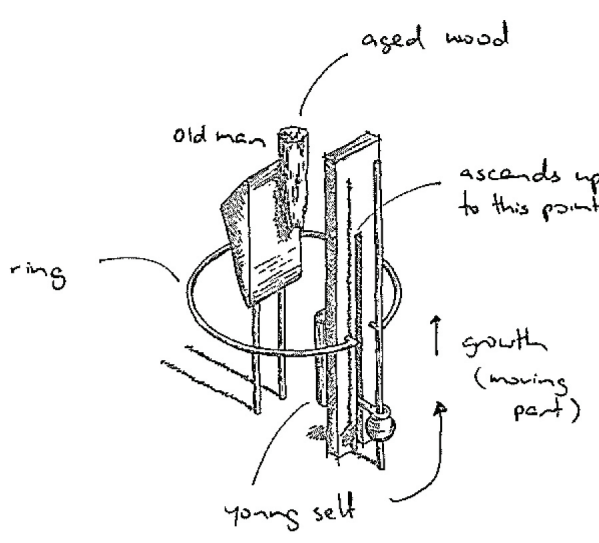

Fig. 20. Initial hand sketch design drawing of superstition 1

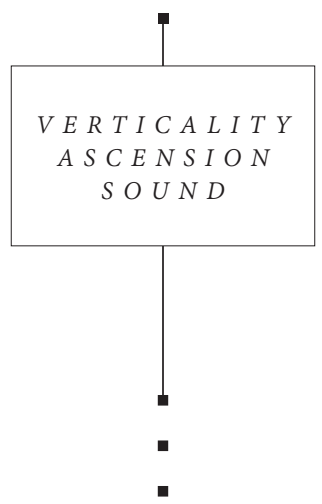

The first conceptual sketch design of the set explores the superstition told to young girls at the dining table that they should not sing while eating as they may grow up to marry an old man.

The abstraction piece consists of two anthropomorphic entities-one depicting the old man as a hunched figure with aged wood as its head and one depicting the young self with its head piece as a moveable kinetic part that sits at the bottom but is able to ascend upwards to come face to face, levelled with that of the old man's. The kinetic part is reflective of the old man's head through a similar cylindrical form but materiality differs through the use of modern plastic as a material reflecting youth whereas decayed wood reflects the old and aged.

The two figures are enveloped and bound within a metal ring reflecting two things: firstly, the notion of a binding marriage and secondly the notion of singing. The ends of the ring also begin from the young entity's side-setting the young self's song as the trigger for the event. 

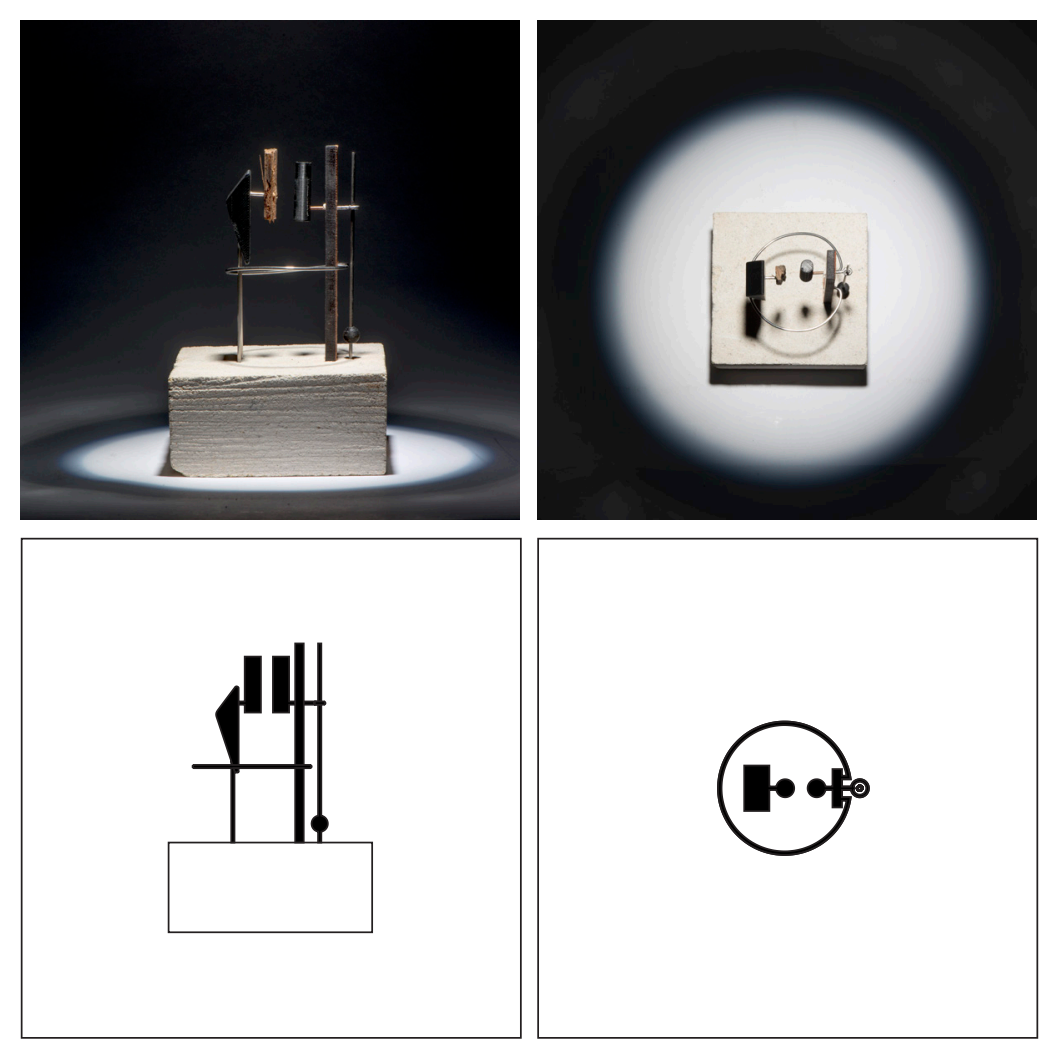

Fig. 21. Singing Old Man: Measured drawings derived from the hand sketches, and converted into a physical model 


\section{ARTEFACT 2}

\section{J. Whistling Snakes}

Whistling at night will invite snakes into one's home.

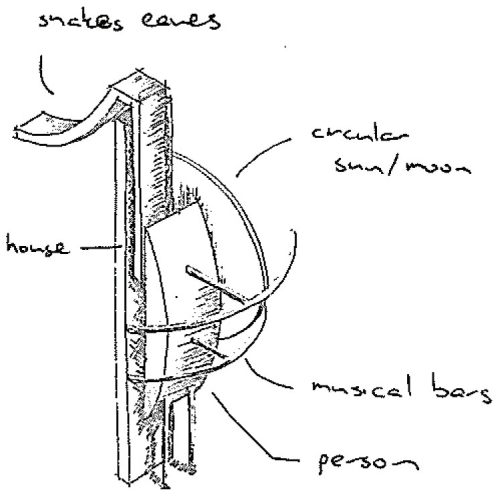

Fig. 22. Initial hand sketch design drawing of superstition 2

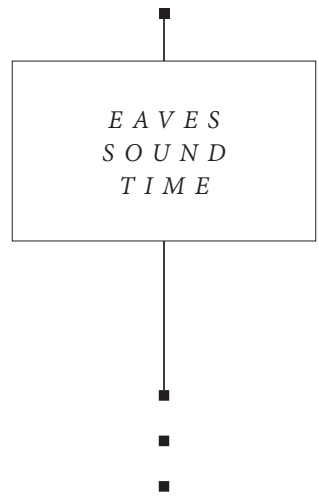

The second conceptual sketch design exploration is of the Whistling Snakes superstition reinterpreting the symbolism of the primordial serpent that appears on the eaves of Eastern temples (Fig. 23). The snakes (Naga) hold sacred meaning and symbolism in Eastern rituals.

The notion of the curved eaves as portrayed in Eastern temples is adopted in this artefact. Although it does not depict an architectural roof, its vertical placement is representative of the placement of an eave relative to the apex of a building.

The musical bars that branch out from the central axis convey the notion of music and whistling emitted from the source that is within the enclosure of the home.

The circular entity depicts a time device-conveying the notion of the event taking place at night time.

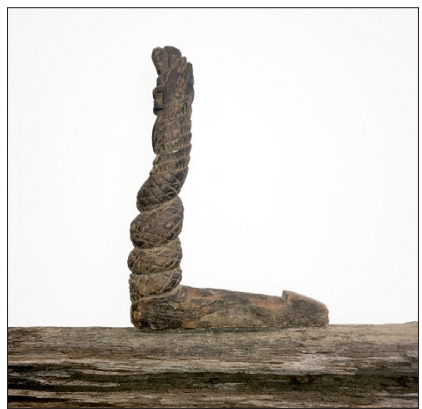

Fig. 23. Photograph of a Wooden Carving of the Naga Serpent-a remnant from the eaves of a Burmese Buddhist Temple. 

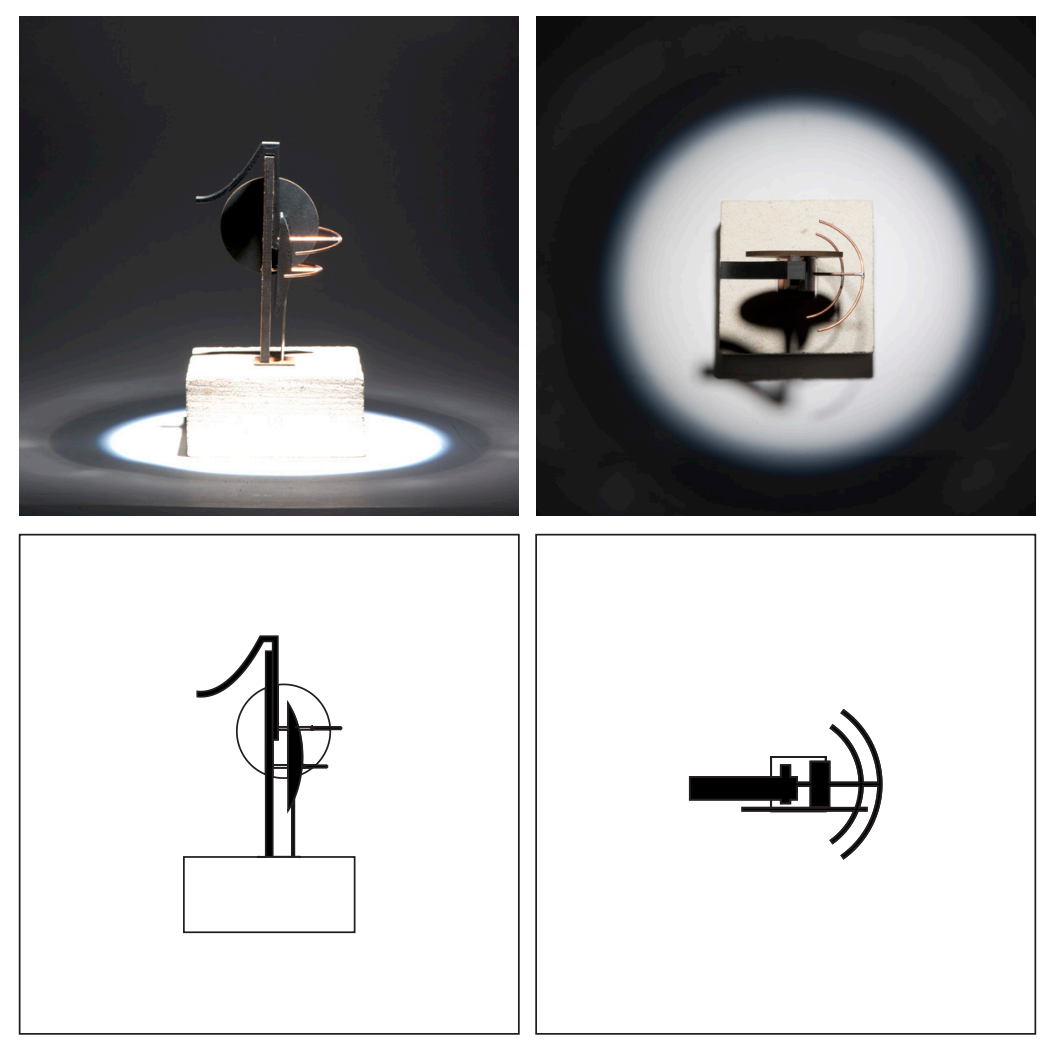

Fig. 24. Whistling Snakes: Measured drawings derived from the hand sketches, and converted into a physical model 


\section{ARTEFACT 3}

\section{Umbrella Ghosts}

Opening umbrellas indoors is an invitation for ghosts.

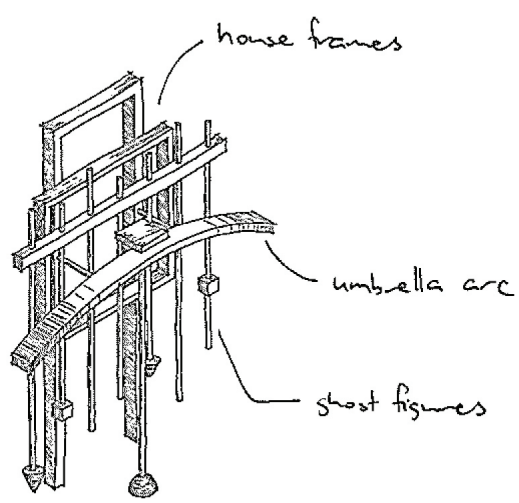

Fig. 25. Initial hand sketch design drawing of superstition 3

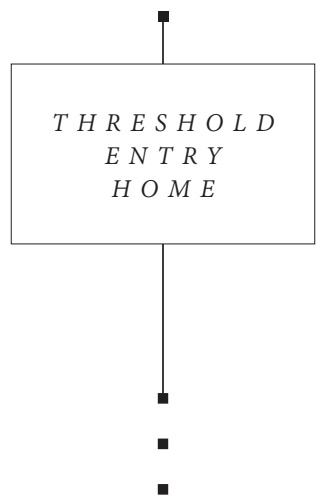

The third conceptual sketch design is based on the superstition that opening umbrellas indoors invites ghosts into the home.

The notion of the home as an envelopment of walls, floor and a roof is abstracted as a 4-sided frame, out of which an arc extends the boundaries of the frame. The arc conveys the umbrella being opened from an internal point. Its ends extending past the frame not being able to fit suggests that it is an act that should not be carried out.

It also depicts the notion of threshold, entry and invitation: the extension of the internal piece towards the external reflects the entry of ghosts into the home through the act of an opened umbrella.

The ghost figures entering the home are conveyed through the arrangement of metal rods passing through the frames. The harsh and cold metal depicts the unpleasantness of the ghosts against a contrast of the warm timber frame of the home. 

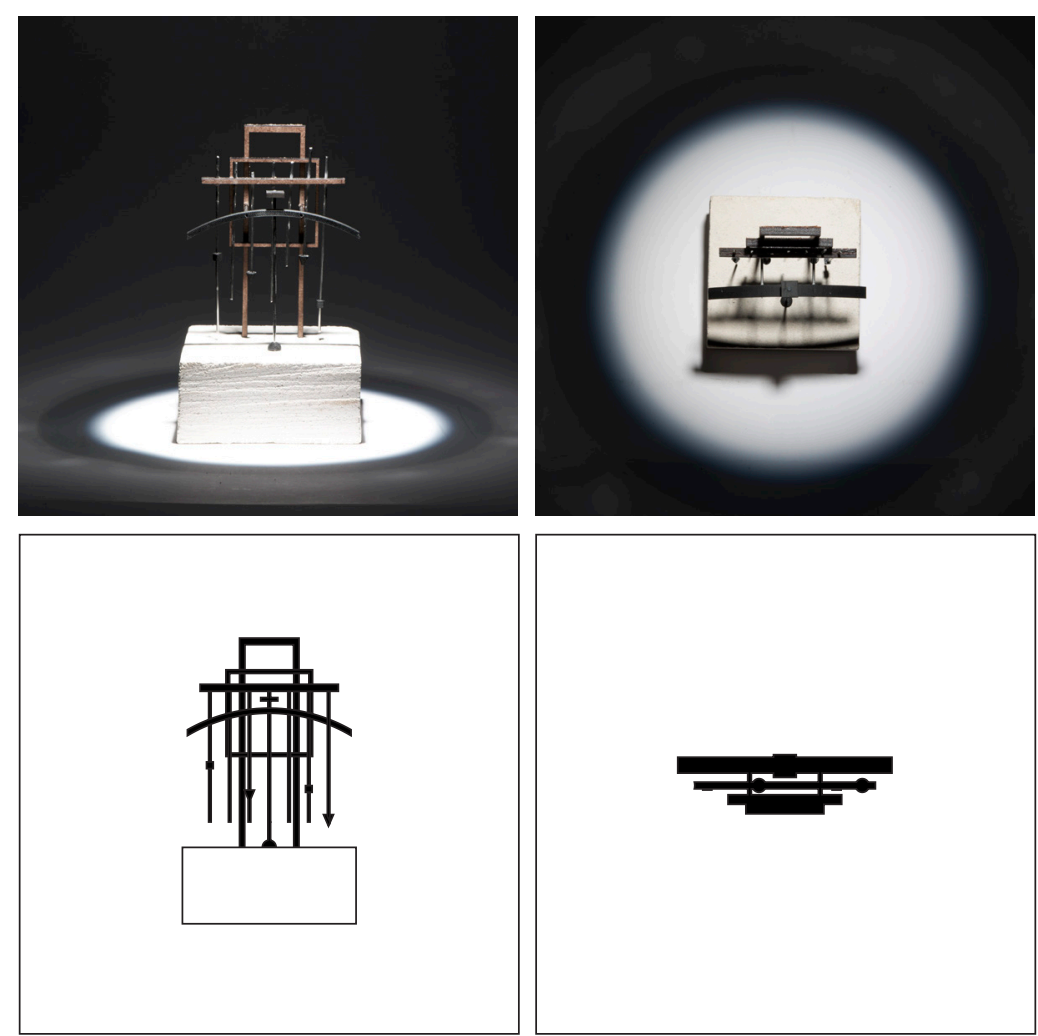

Fig. 26. Umbrella Ghosts: Measured drawings derived from the hand sketches, and converted into a physical model 


\section{ARTEFACT 4}

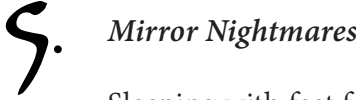

Sleeping with feet facing a mirror will incite bad dreams.

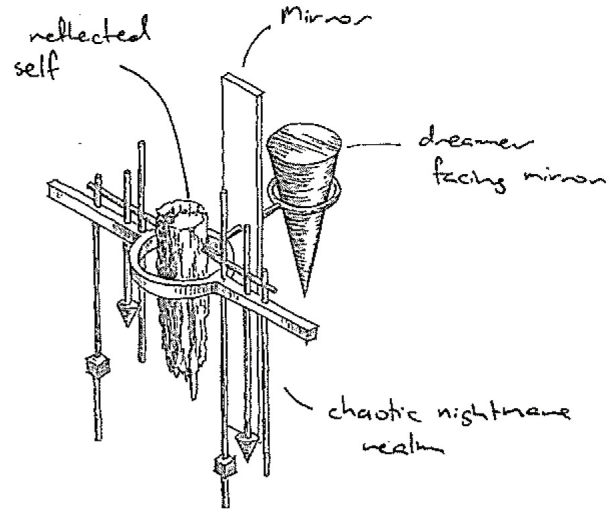

The fourth conceptual sketch design is based on the superstition that sleeping with feet oriented towards a mirror will incite bad dreams.

The abstracted piece conveys two entities on separate sides of a clear sheet representing a mirror: one side is the dreamer depicted by a clean cone-like form, calm and tranquil; the other side is the reflected side of the dreamer in a chaotic nightmare realm. The contrast in choice of materiality of the two sides clearly distinguishes the calm and tranquil side from the nightmarish chaotic side.

Fig. 27. Initial hand sketch design drawing of superstition 4

The two doubles are clear representations of each other in different states.

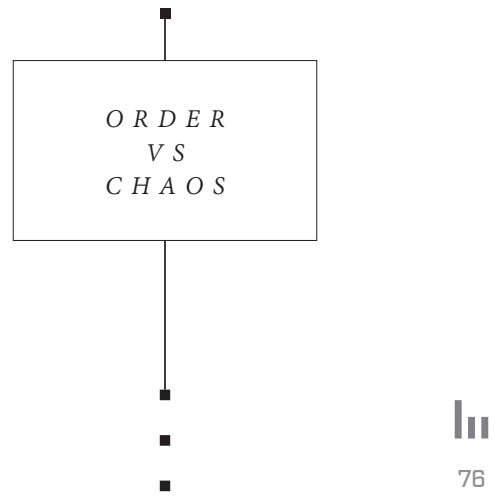



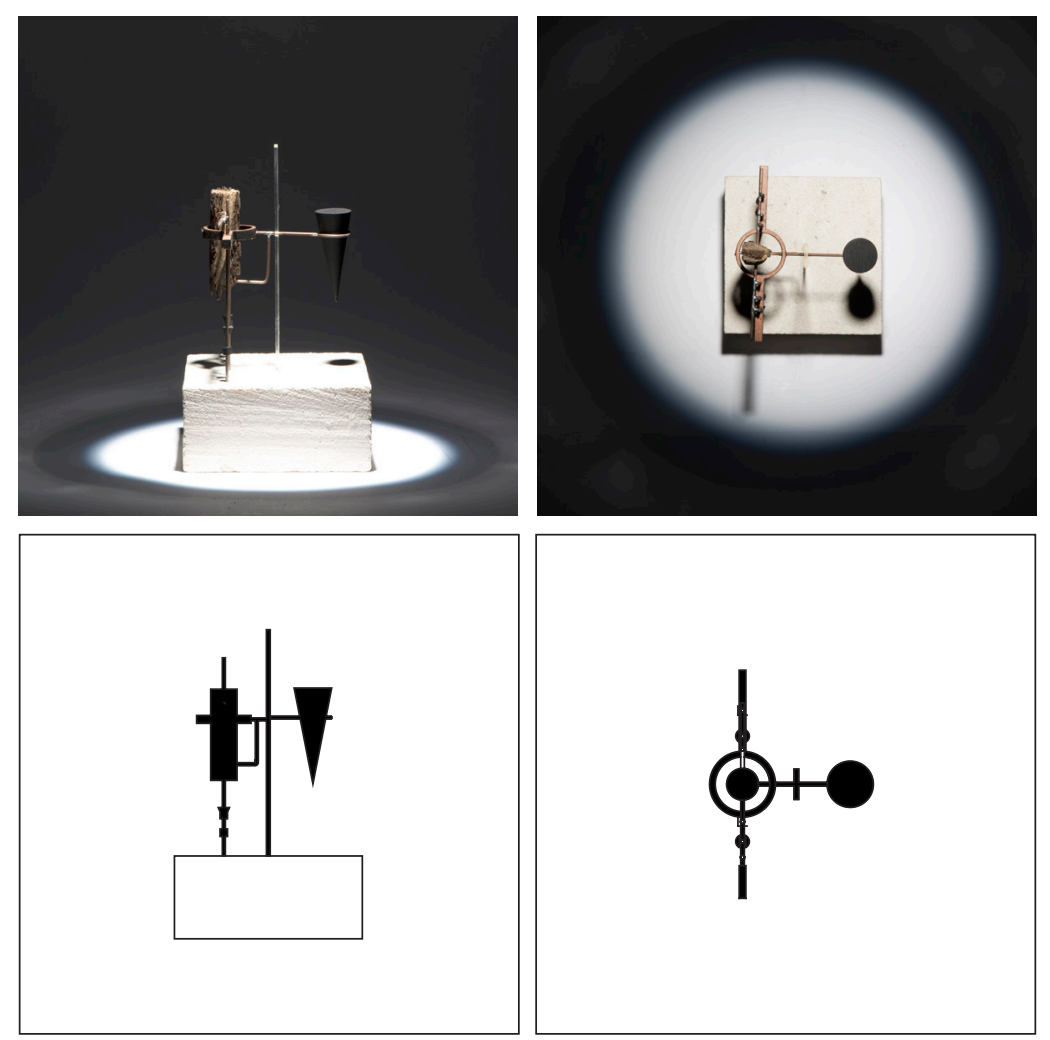

Fig. 28. Mirror Nightmares: Measured drawings derived from the hand sketches, and converted into a physical model 


\section{ARTEFACT 5}

\section{Fingernail Ghosts}

Clipping nails at night will provoke ghosts.

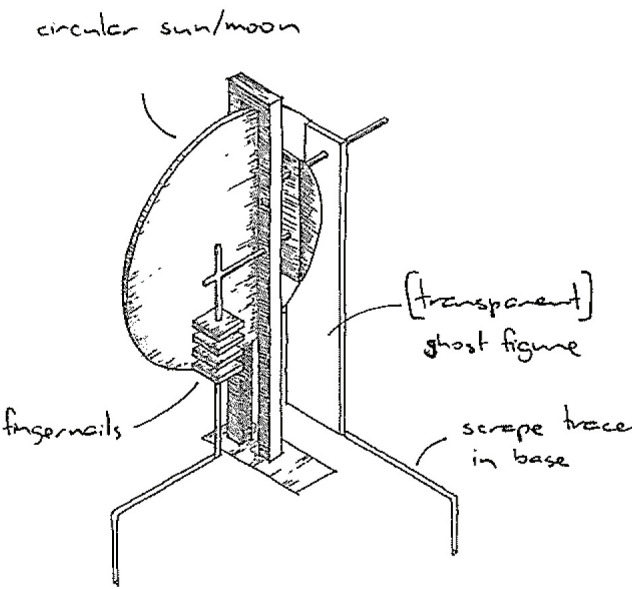

Fig. 29. Initial hand sketch design drawing of superstition 5

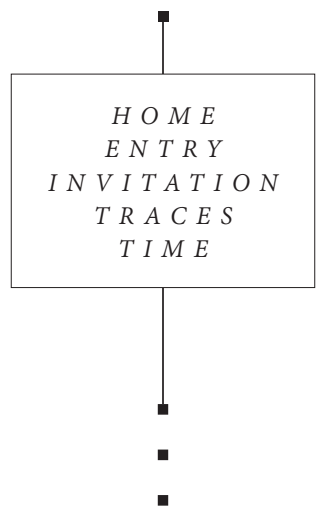

The fifth conceptual sketch design is based on the superstition told to children to discourage them from clipping nails at night as it may provoke ghosts and invite them into the home.

There are 4 pieces hanging in mid-air implying that they may drop at any moment. This is a suggestion towards the 5 fingernails of a hand and that one fingernail has already dropped-clipped thus triggering the notions of entry, invitation and threshold carried out by the ghost figure.

The ghost figure is depicted through a transparent acrylic sheet and it demonstrates implied movement through the carved out traces it leaves behind in the limestone base. This notion implies the dynamic movement of the ghost figure where it entered from outside the boundary of the piece and ends up in the centre of activity.

The circular entity also depicts a time device-conveying the notion of the event taking place at night time. 

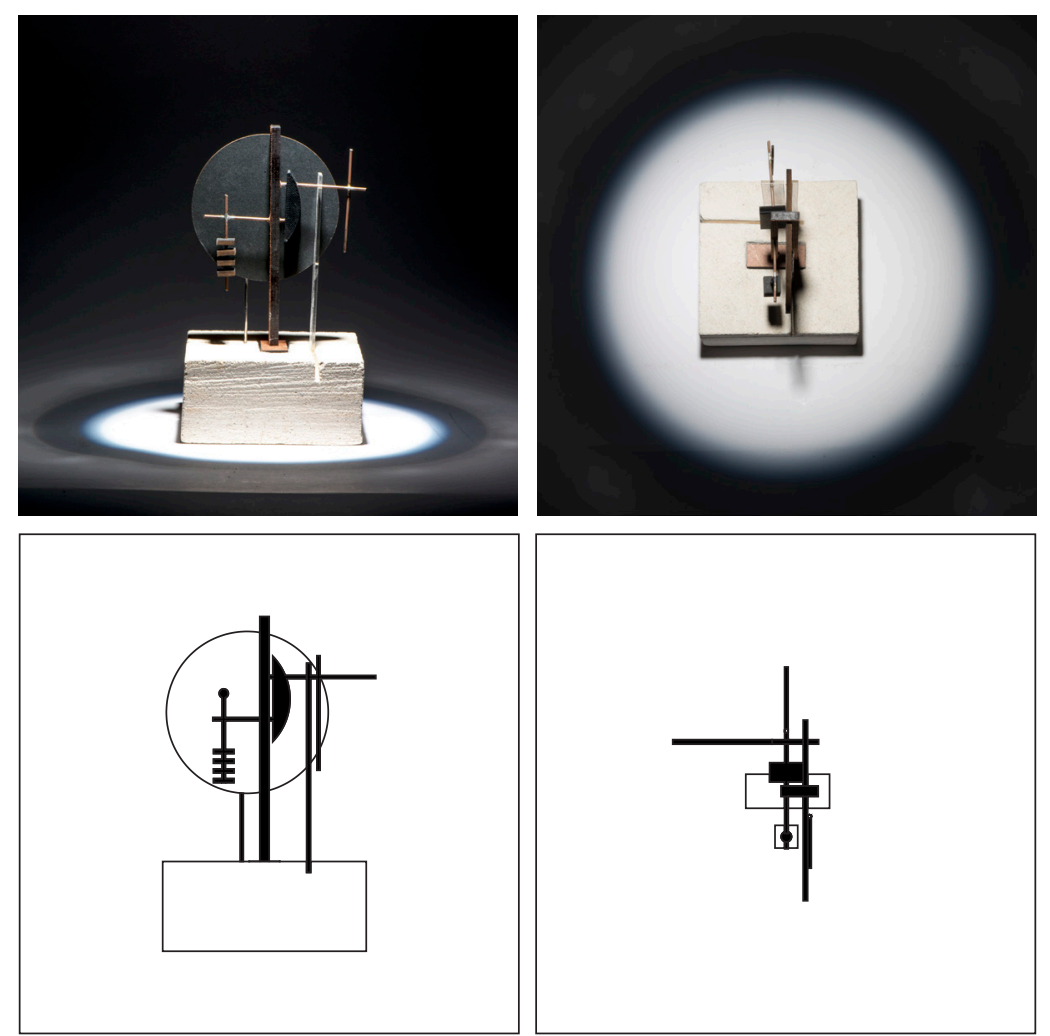

Fig. 30. Fingernail Ghosts: Measured drawings derived from the hand sketches, and converted into a physical model 


\section{ARTEFACT 6}

\section{Baby Tooth}

Throw broken baby tooth upwards when it's a bottom tooth or downwards when it's the top so that the new teeth will know which way to grow.

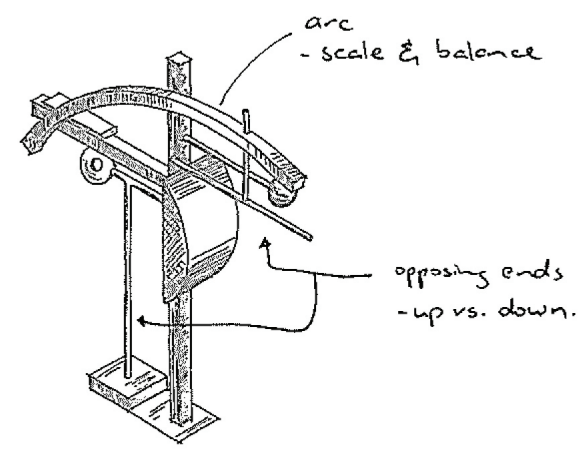

Fig. 31. Initial hand sketch design drawing of superstition 6

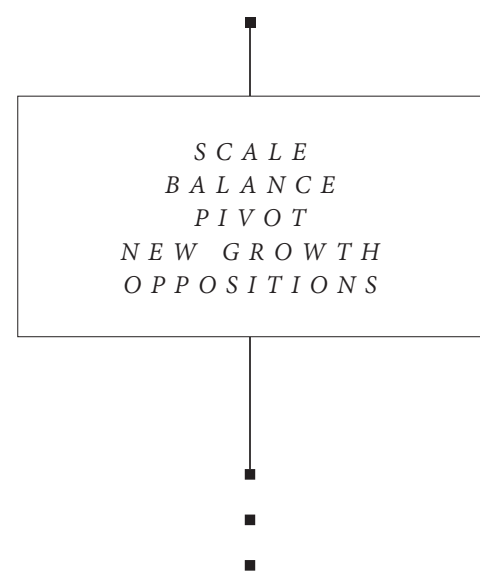

The sixth conceptual sketch design translates the concept of the growth of baby teeth indicated by the direction the broken tooth is thrown into. The notion that the bottom tooth needs to be thrown up and the top tooth needs to be thrown down is abstracted into the idea of scale and balance.

The abstracted piece conveys this idea through the arc centered on the central axis as a rotational pivot, and the constituent elements on opposing ends convey opposing vertical implications: one side directed towards ascension and one side directed towards descension. 

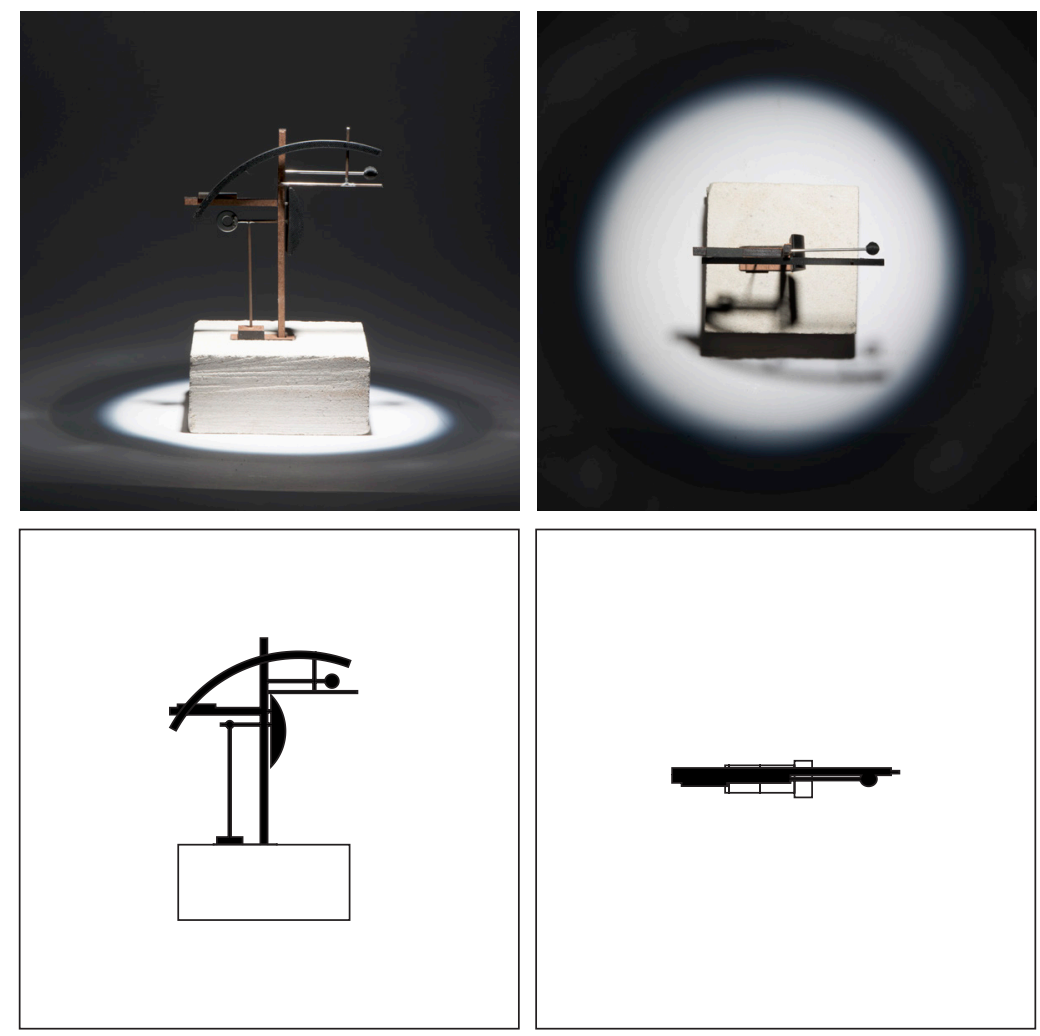

Fig. 32. Baby Tooth: Measured drawings derived from the hand sketches, and converted into a physical model 


\section{ARTEFACT 7}

\section{- Elbows of Misfortune}

Don't rest elbows on the dining table, or else one may end up poor.

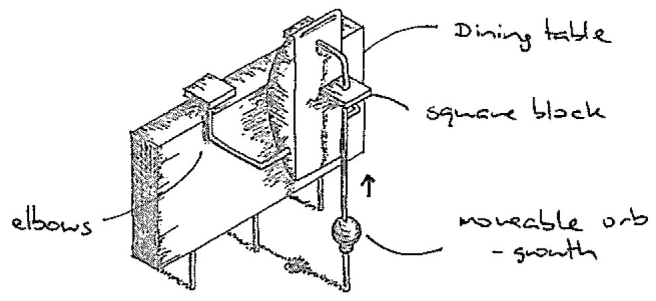

Fig. 33. Initial hand sketch design drawing of superstition 7

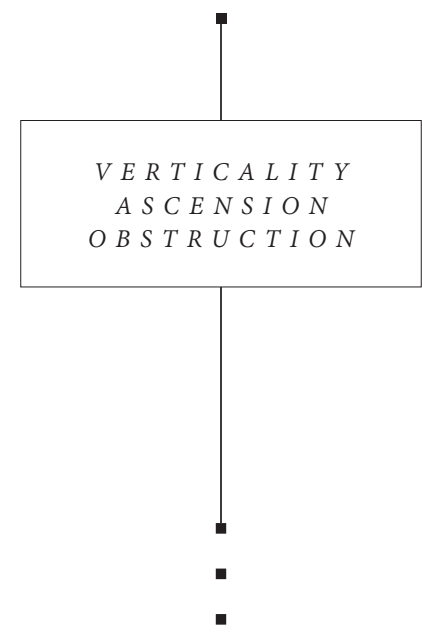

The seventh conceptual sketch design is based on the superstition told to children to not rest their elbows on the dining table as this can lead to one living a life of misfortune.

The idea taken from this is the notion of a stunted growth in one's financial life. The abstraction piece translates the concept of the stunted growth through a moving kinetic piece that is able to ascend only up to a certain point where its ascension is hindered by an obstacle.

The formal construct of the piece takes on the abstracted appearance of an anthropomorphic entity with its elbows resting upon a table. The kinetic piece is housed on a vertical rod that supports the anthropomorphic entity to suggest that the stunted growth and the entity's resting of elbows are interrelated events. 

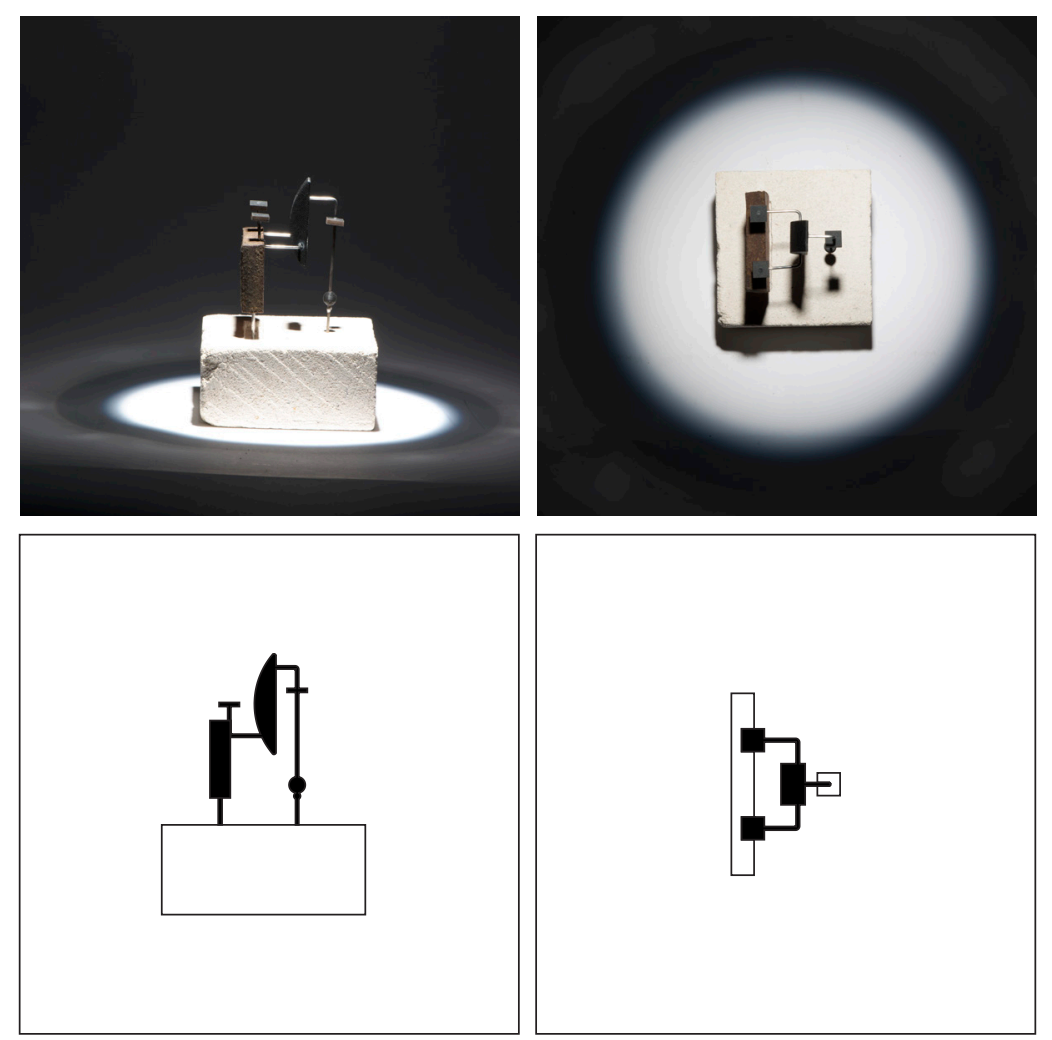

Fig. 34. Elbows of Misfortune: Measured drawings derived from the hand sketches, and converted into a physical model 


\section{ARTEFACT 8}

\section{ภ. Twitching Eye}

A twitching eye means someone is missing them.

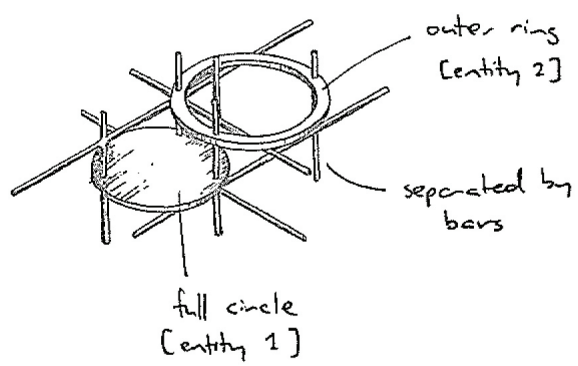

Fig. 35. Initial hand sketch design drawing of superstition 8

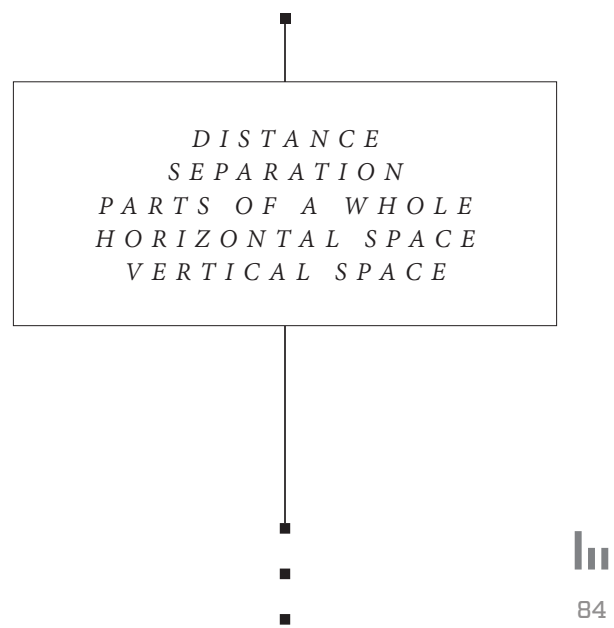

The eighth conceptual sketch design is based on the superstition that the twitching of an eye is an indication that someone is missing you in that moment.

The formal construct of the abstracted piece consists of two circular entities representing the eyes as well as two beings that are missing each other. Entity one is a full circle whereas entity two appears as an outer ring from which the inner circle that is entity one has been removed-indicating that the two were once complete together and now they have been shifted apart by vertical and horizontal bars. 

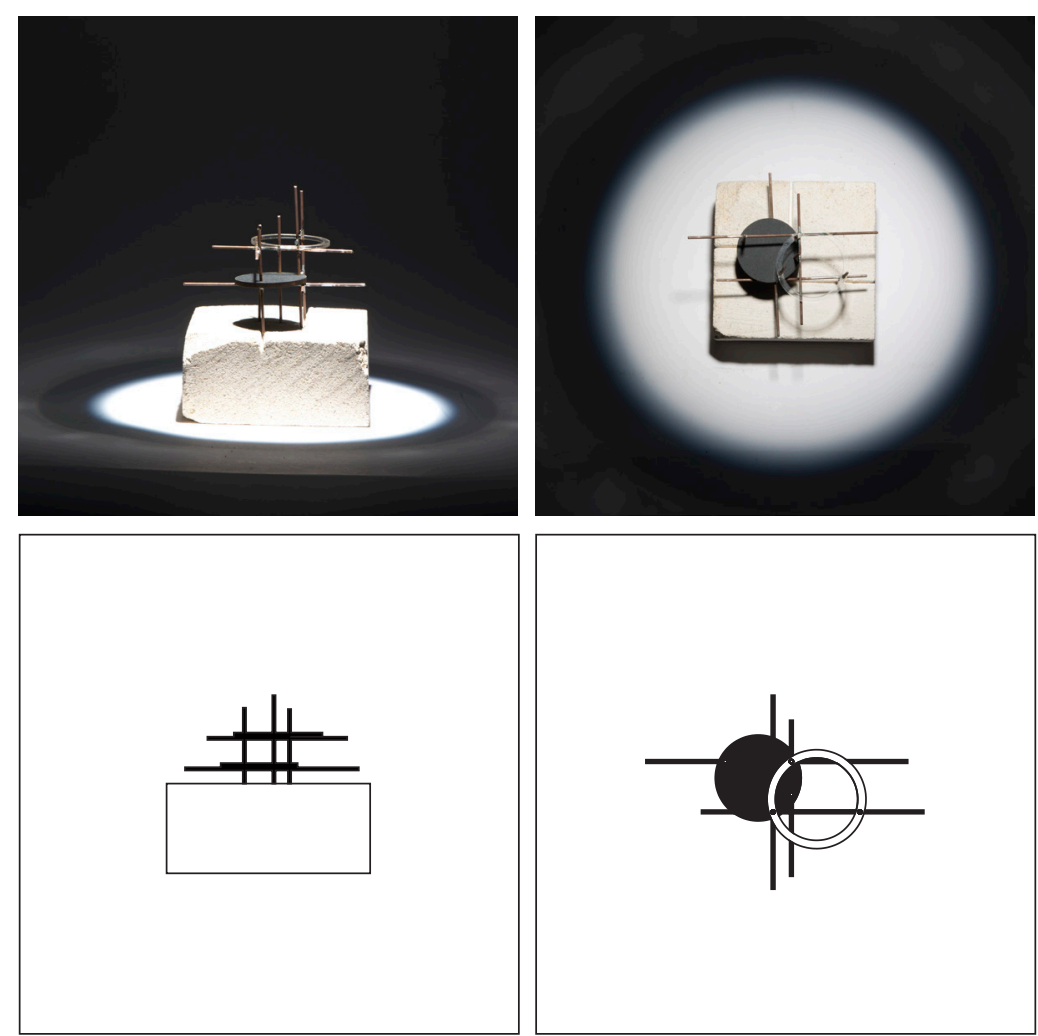

Fig. 36. Twitching Eye: Measured drawings derived from the hand sketches, and converted into a physical model 


\section{ARTEFACT 9}

c. Sleep Paralysis

When one experiences sleep paralysis, it is because a ghost is sitting on them.

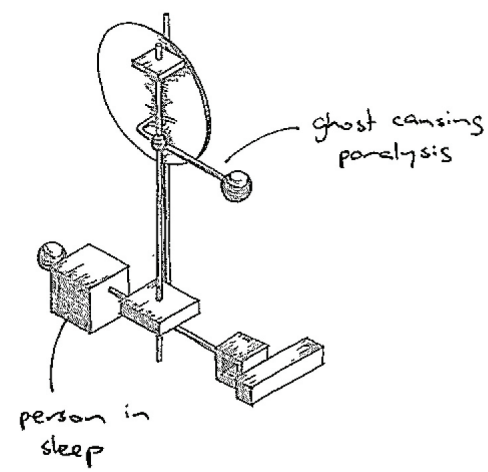

Fig. 37. Initial hand sketch design drawing of superstition 9

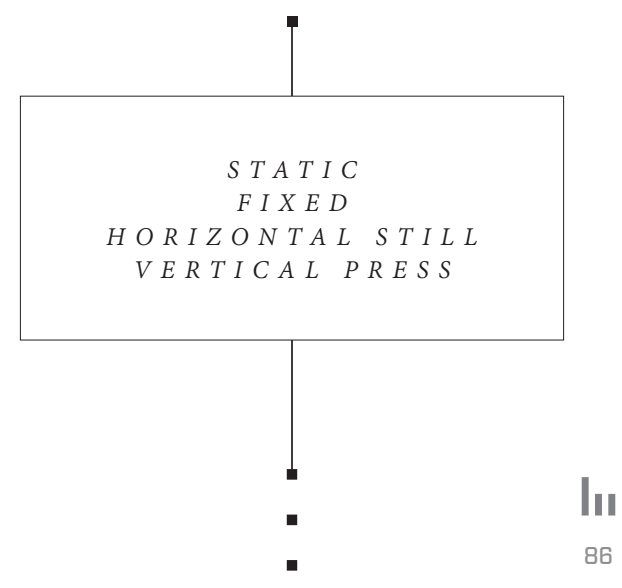

The ninth conceptual sketch design is based on the superstition that the pressing feeling one experiences during sleep paralysis is caused by a ghost figure pressing down upon them.

The abstraction piece is conceived as two anthropomorphic entities: one horizontal entity that is the sleeper; and one vertical entity that is the ghost figure pressing upon the sleeper rendering them unable to move. 

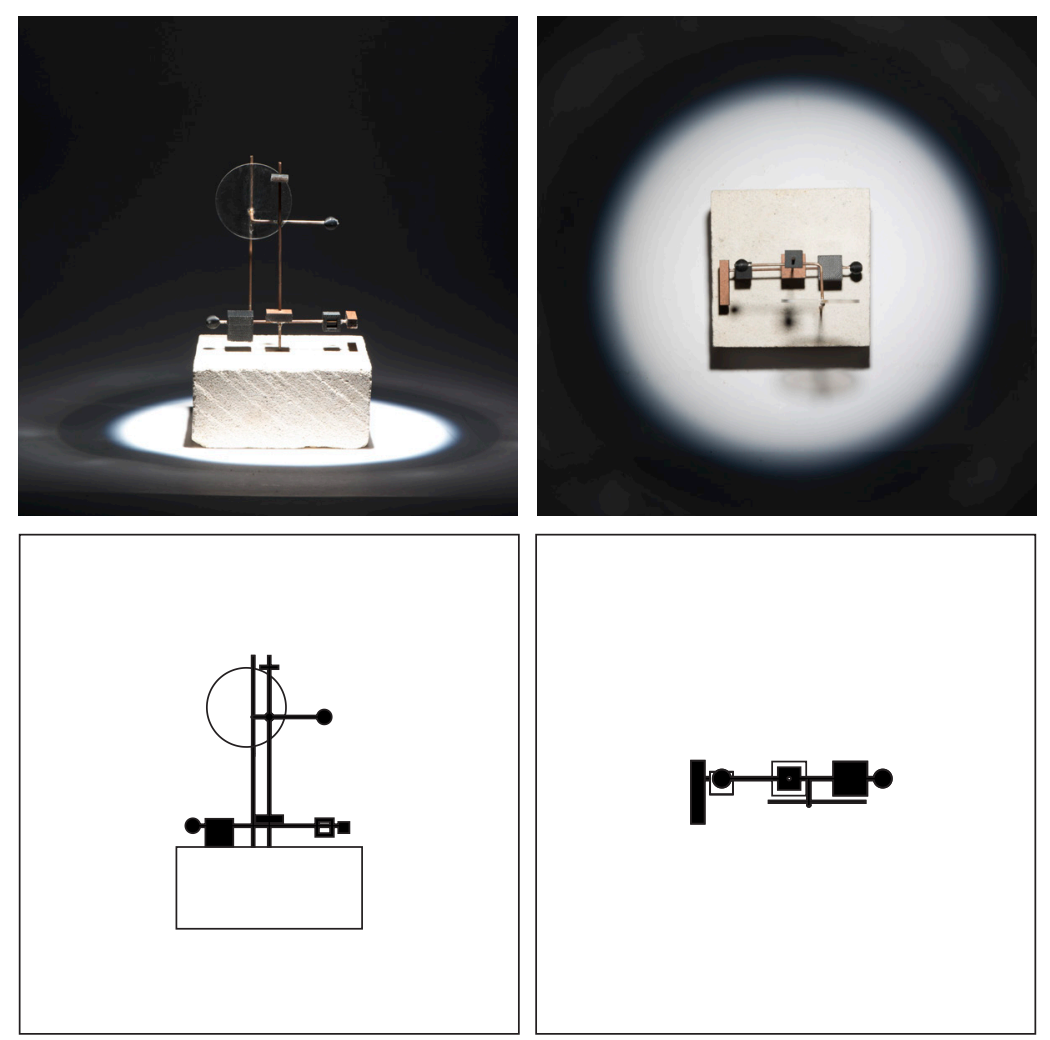

Fig. 38. Sleep Paralysis: Measured drawings derived from the hand sketches, and converted into a physical model 



\section{Further Explorations}

The following additional drawing explorations were undertaken to interrogate how the formal outcomes of the artefacts can lead towards unravelling spatial outcomes, architectural elements and conceptual frameworks for moving the project further in the next stage.

The drawings interrogate how the formal outcomes can be redefined and reinterpreted as architectural elements-wall, floor, threshold, etc. Through the use of an evocative starting point of superstitions, the results showcase a range of reinterpretations that begin to deviate away from Eurocentric modernist principles and constructs as set out per RO 1:

To explore how culturally scripted ideas can be explored and expressed through allegorical methods in the conception of an evocative architectural framework that deviates from Eurocentric modernist principles and constructs. 
it

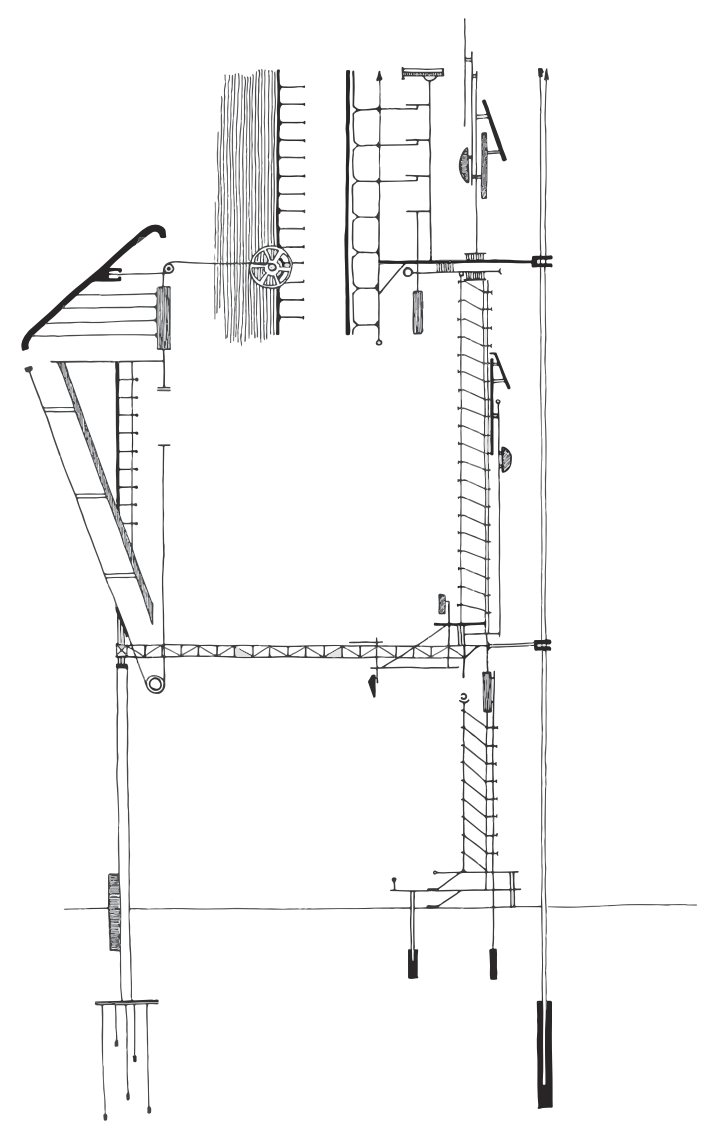




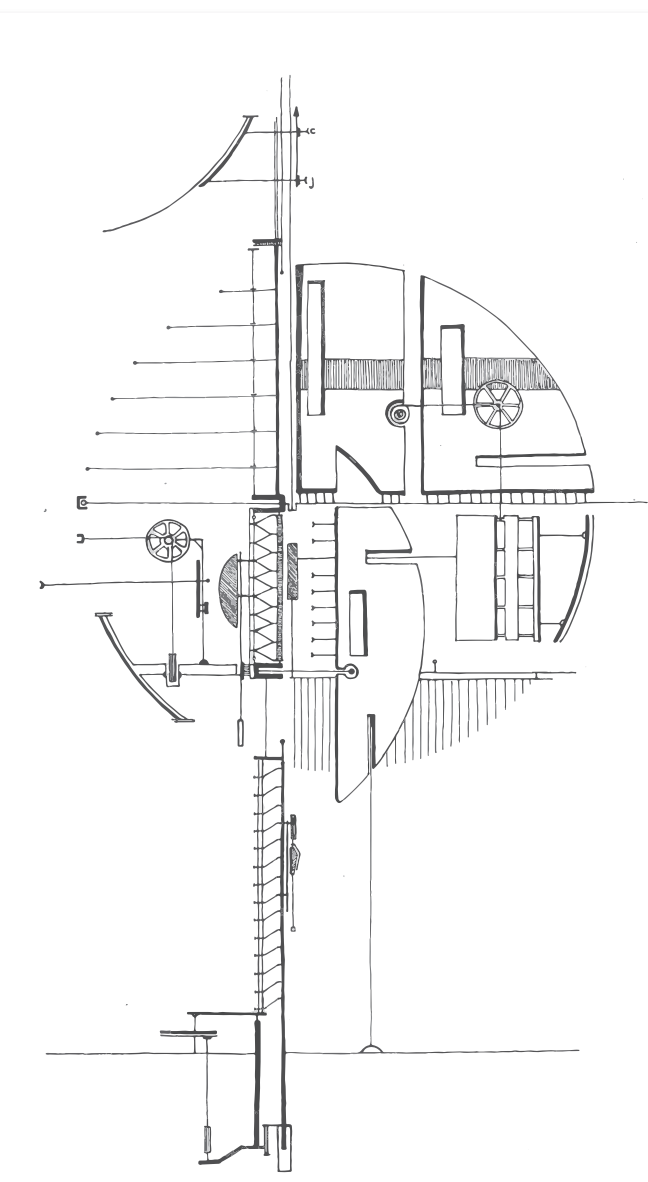


舤

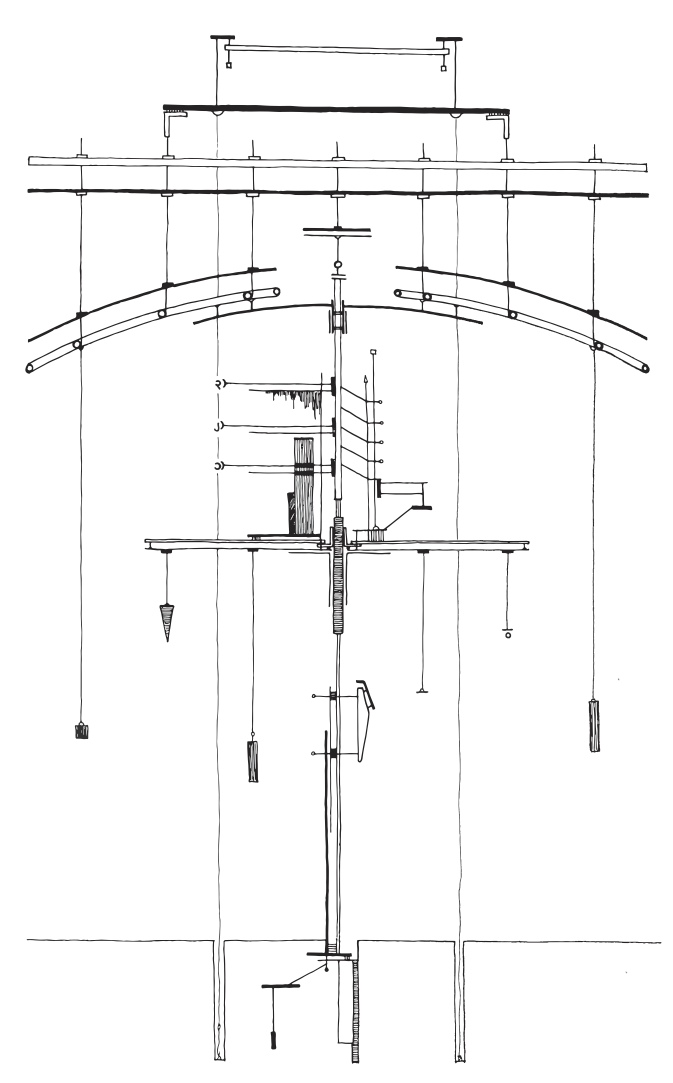


tr

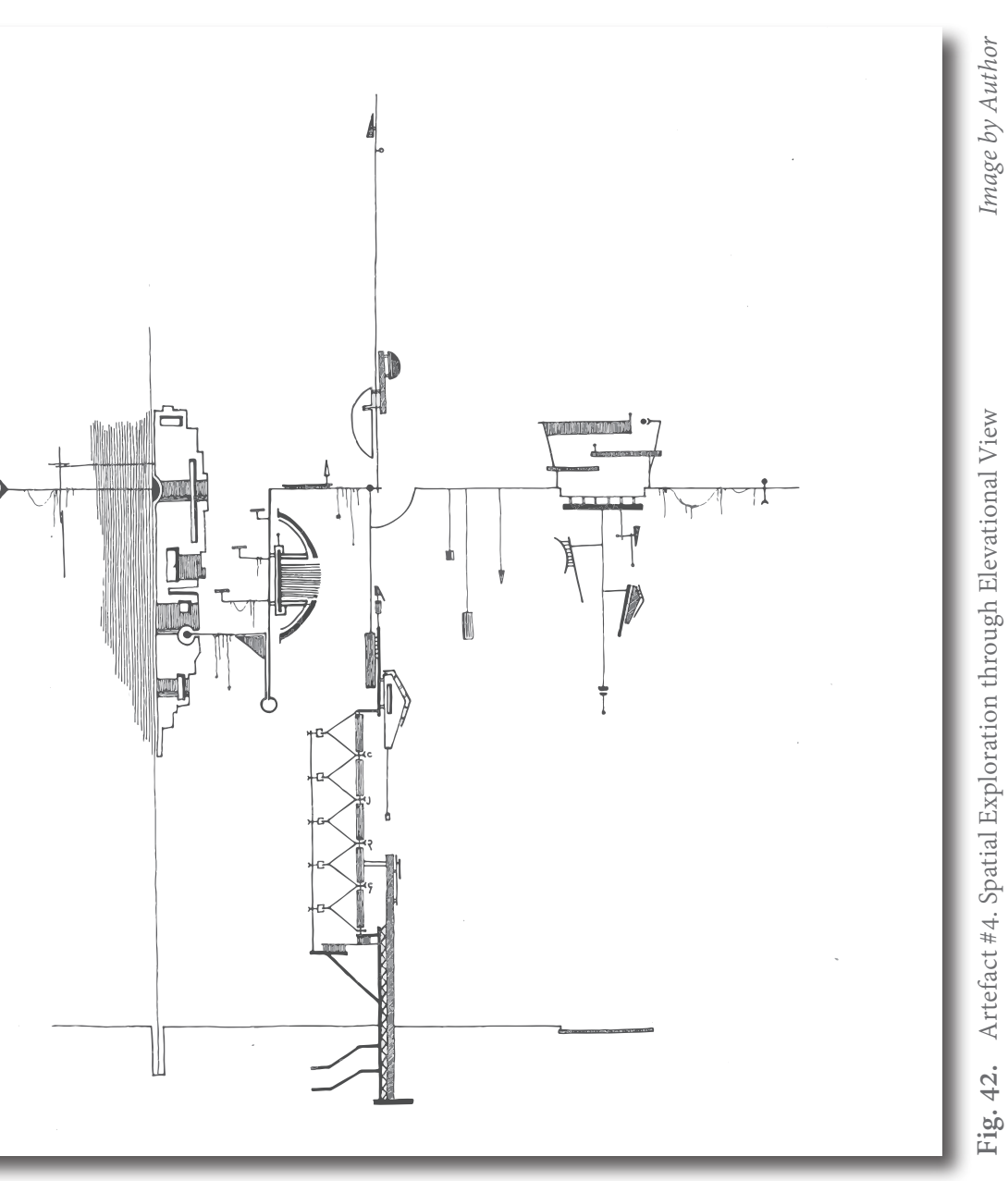


Aft

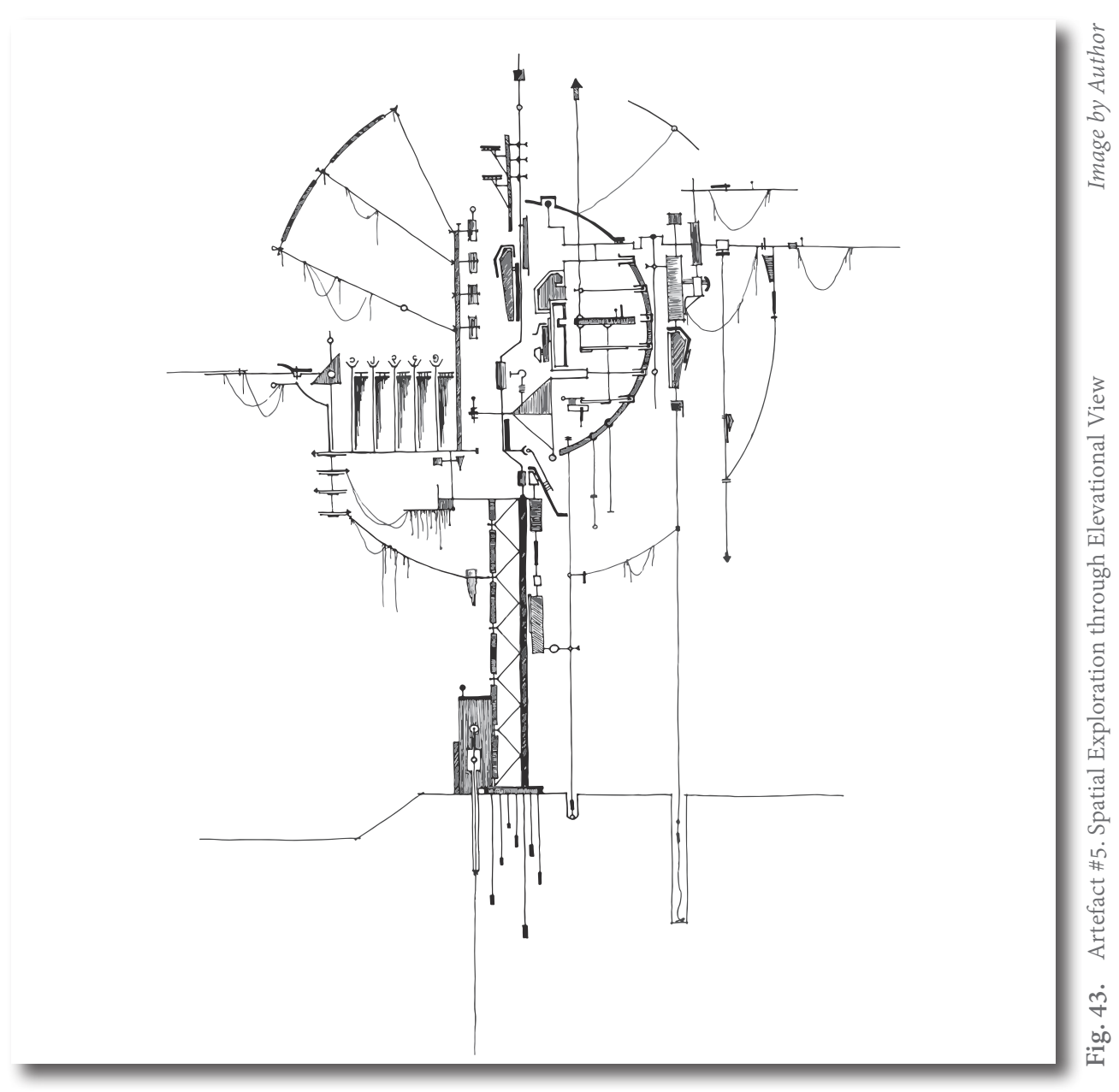


9it

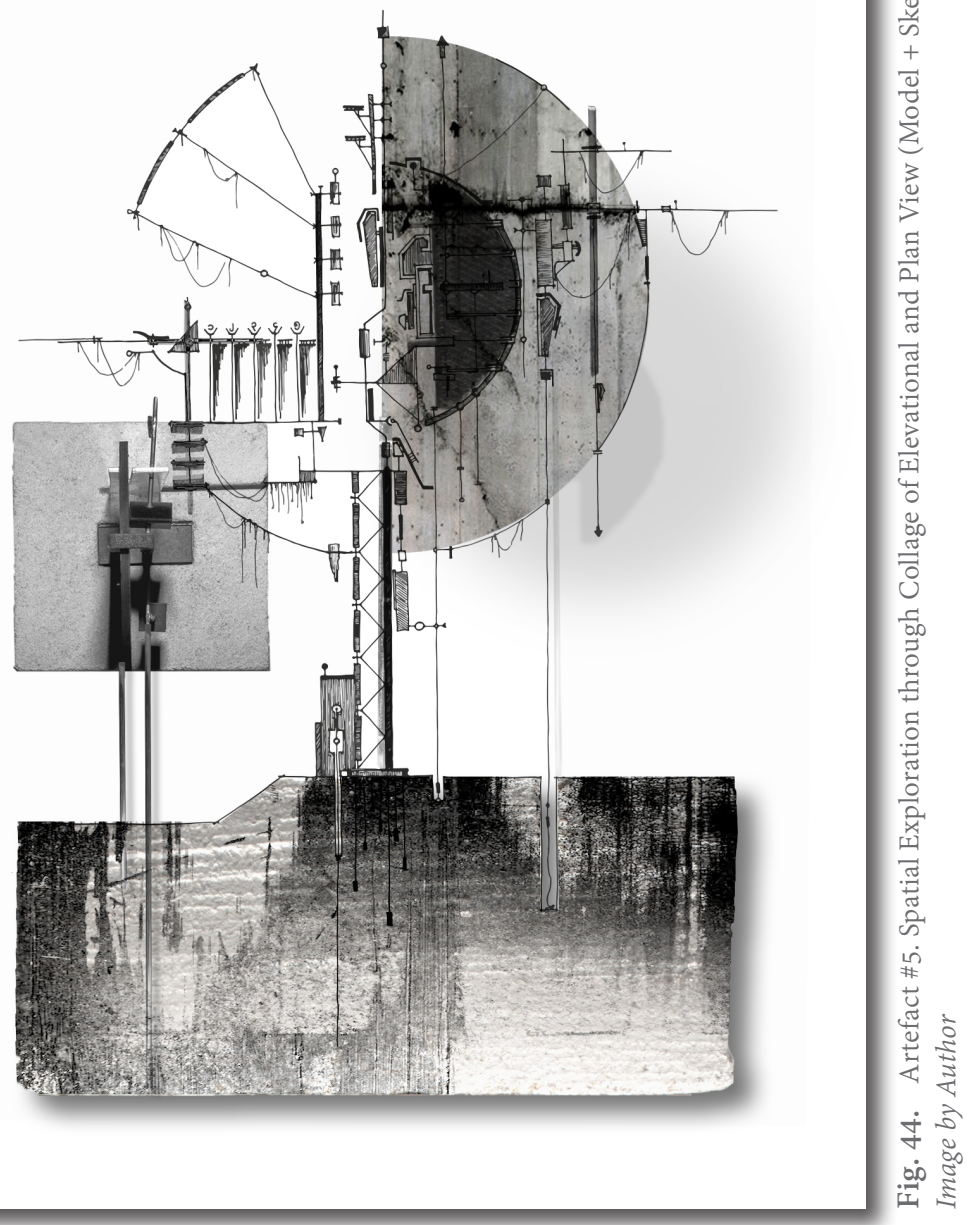


ANIMATED SKETCH CONCEPT

This animated sketch concept explored dynamic relationships of its constituent parts. The rotational parts cross over at certain points in time but some never come to meet.

This concept led the project to head towards a digital game environment where the players in the navigation of the virtual space encounter a different experience each time for every participant.

96 


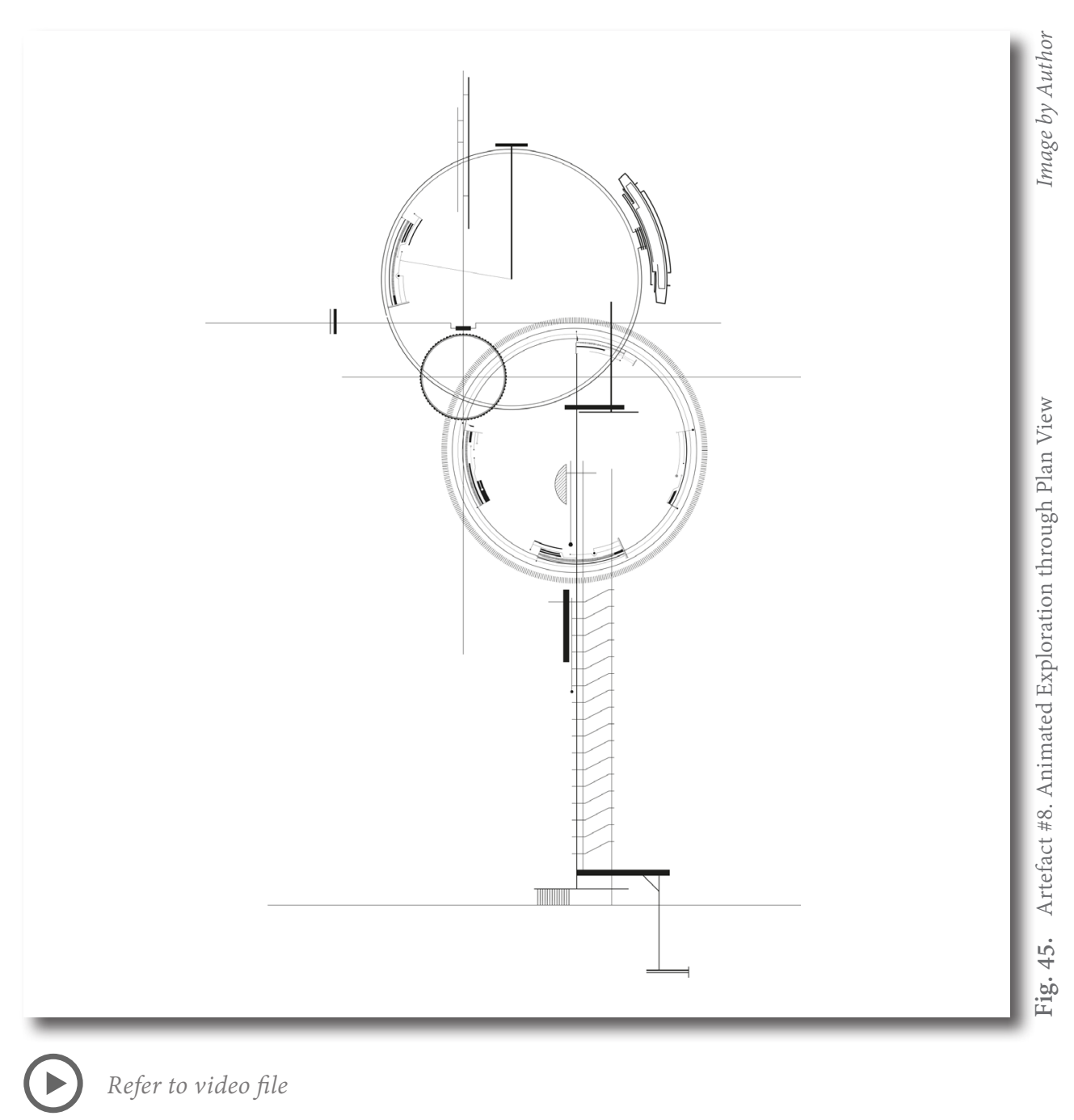


9 Artefact Models

The nine architectural artefact conceptual sketch models are arranged to coexist together as a conceptual framework to help enable the concept sketch designs to transition from the physical realm towards the digital environment in Design Stage 2.

The 9-square grid arrangement was selected to organise the set, and the grid continues through to Design Stage 3 as a collective whole within a game environment.

The 9 artefacts are arranged in a $3 \times 3$ grid by their numerical order-a layout that is consistent throughout the exploration series.

III

98 


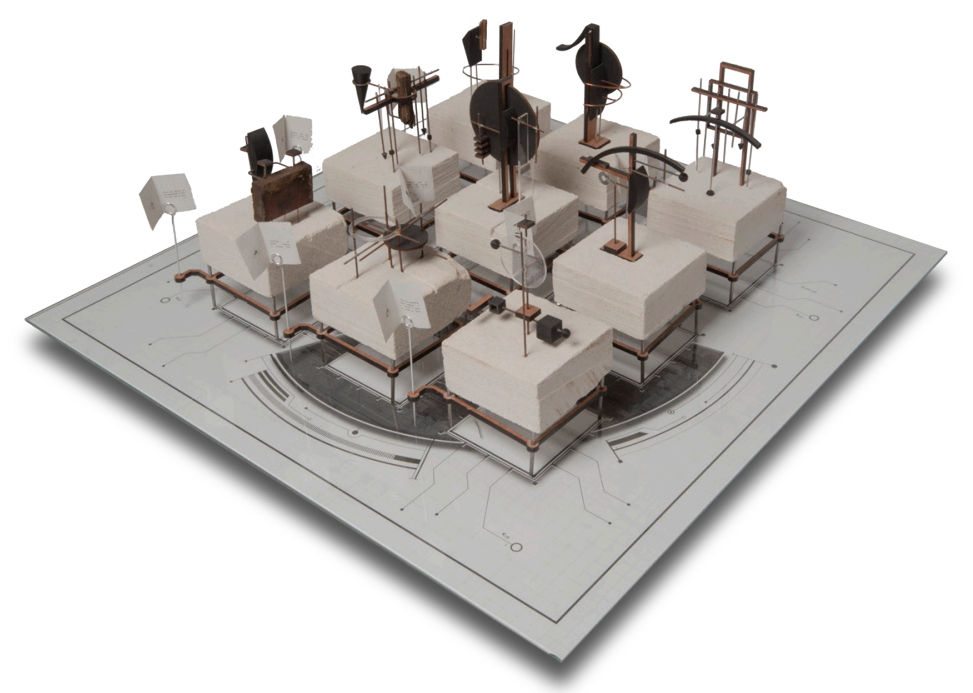

Fig. 46. 9 Model Artefacts on Model Plinth Base 



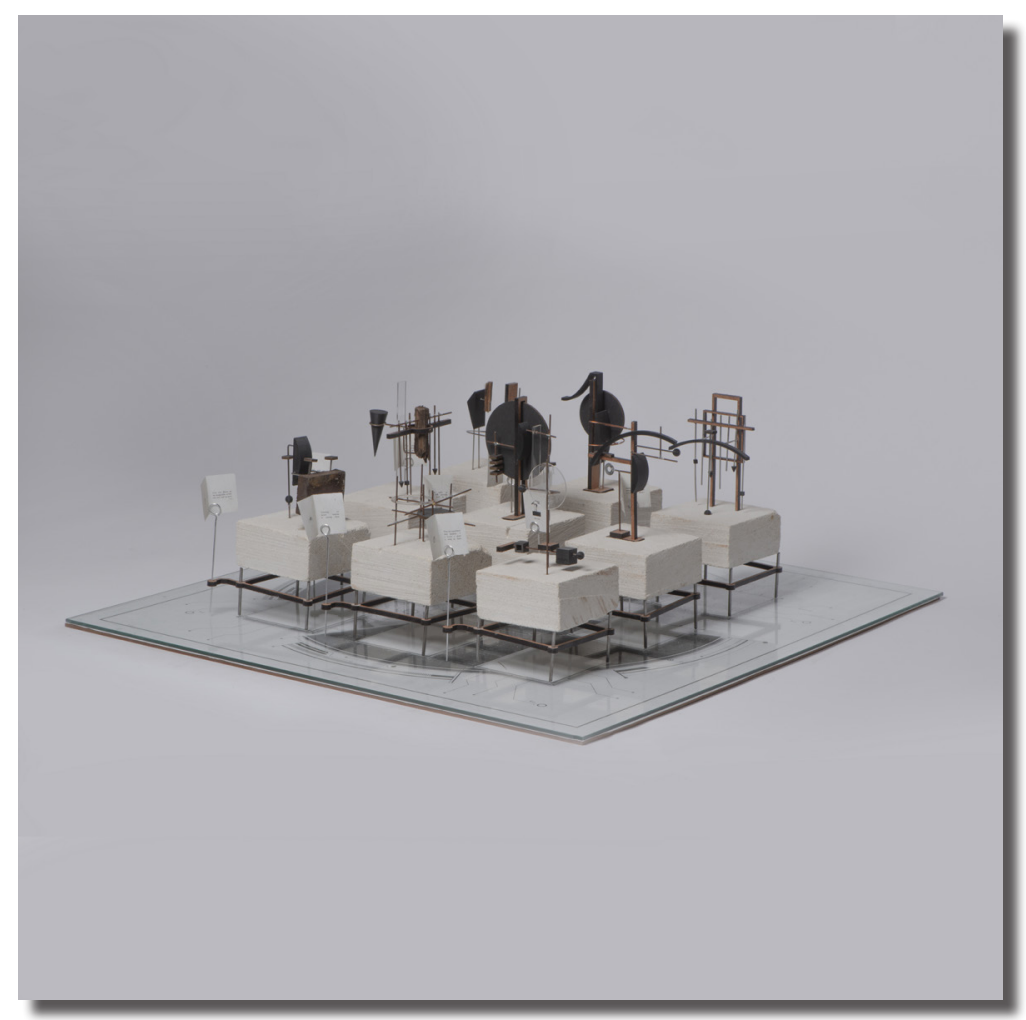

Fig. 47. 9 Model Artefacts arranged in a Grid 
This early conceptual masterplan (Fig. 48) was drafted to interrogate how each of the 9 pieces could become intervention pieces/inhabitants of a digital game environment in the final Design Stage 3. The drawings search for methods for how each piece could create relationships with another and how a player could transition spatially from one to the other.

The dynamic implications of the base drawing searches for ways that the game environment could shift, altering spatial conditions and creating new spatial-player relationships. 


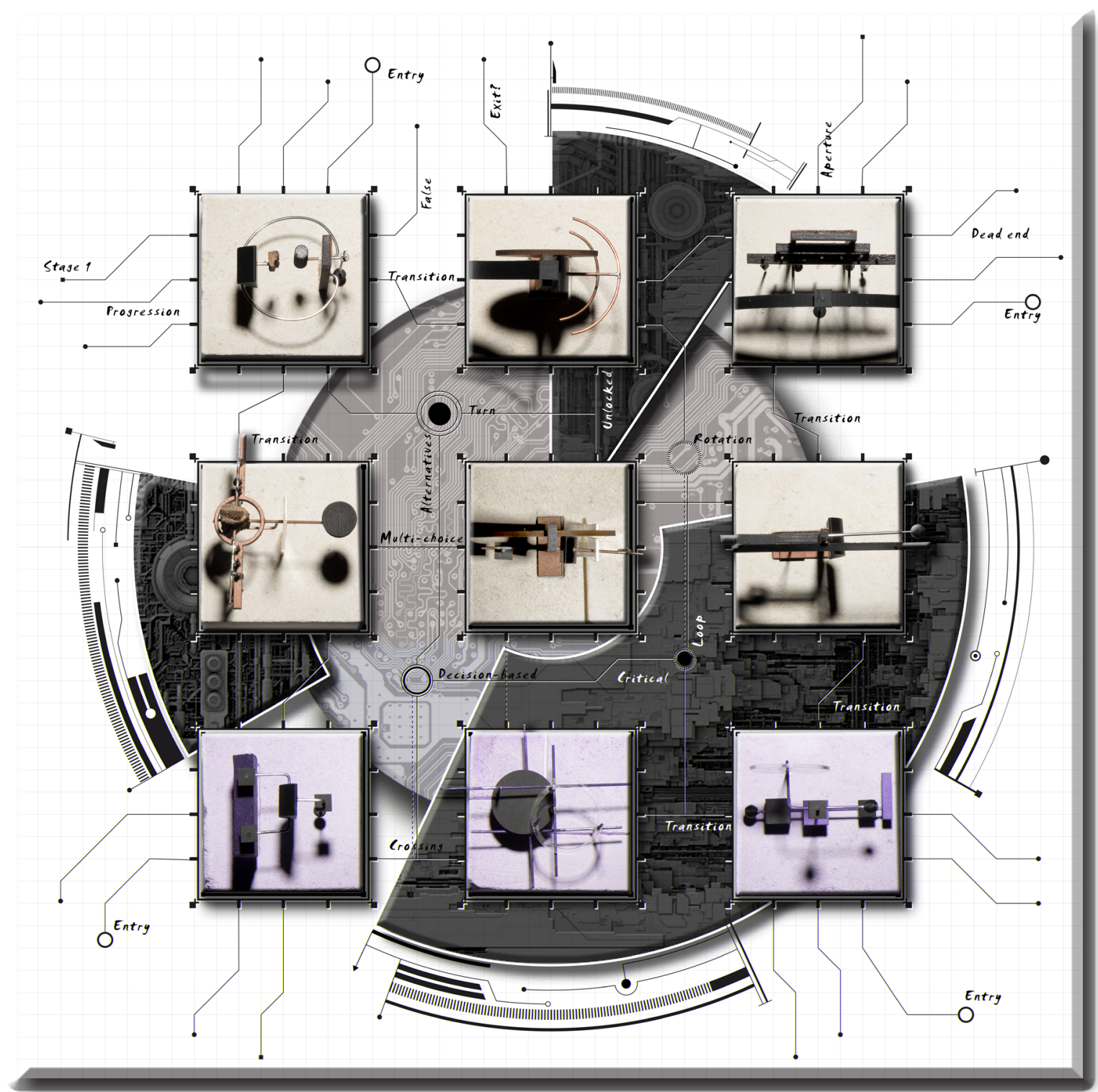

Fig. 48. Early Conceptual Game Masterplan using the 9 Model Artefacts 
This notational diagram (Fig. 49) extracts the formal outcomes of the 9 artefact models from an elevational perspective and abstracts them into line drawings. The 9 grids organise the drawings using a system of arcs and lines to draw links and connections using the similar themes they each convey. This system of links implies dynamic shifts of how the spaces can be altered over time.

The grids suggest a fabricated context-a virtual digital site upon which the 9 interventions can be situated where they can coexist in a system organised

by their links and connections. 


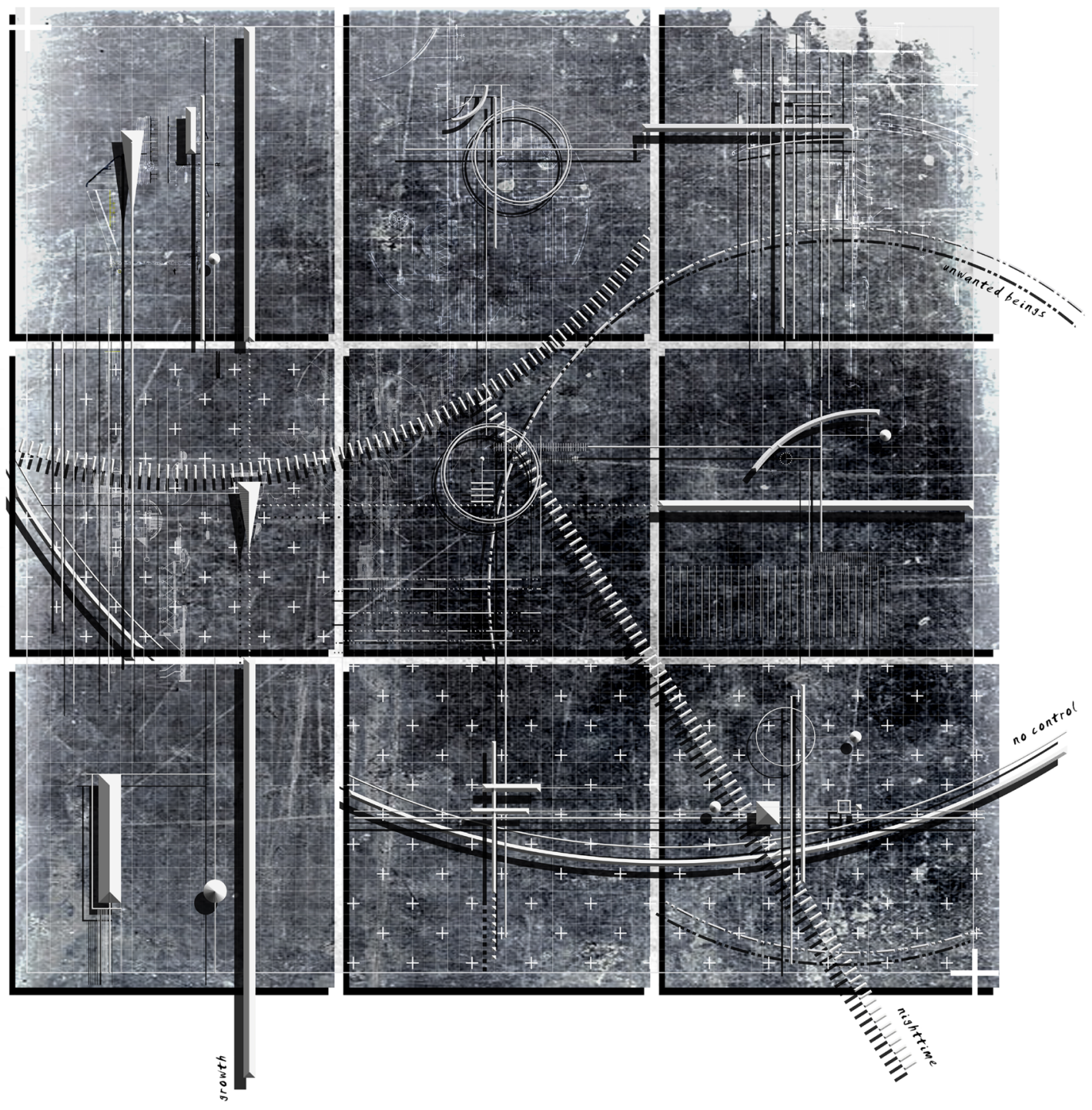

Fig. 49. Conceptual Notation Diagram Drawing Links between 9 Artefacts [\#1. Abstraction through Elevational view] Image by Author 
This notational diagram is a replica of the previous diagram (Fig. 49) - the difference is that the abstracted drawings of the 9 artefacts are extracted from their plan views.

This exploration exhibits new outcomes and possibilities arising through the altering of perspective. This gives more opportunities for how the spaces are constructed in the next stage based on how the artefacts are viewed as well as contribute to the notion of the constant shift of the individual's perception as a construct of a personalised architectural narrative experience. 

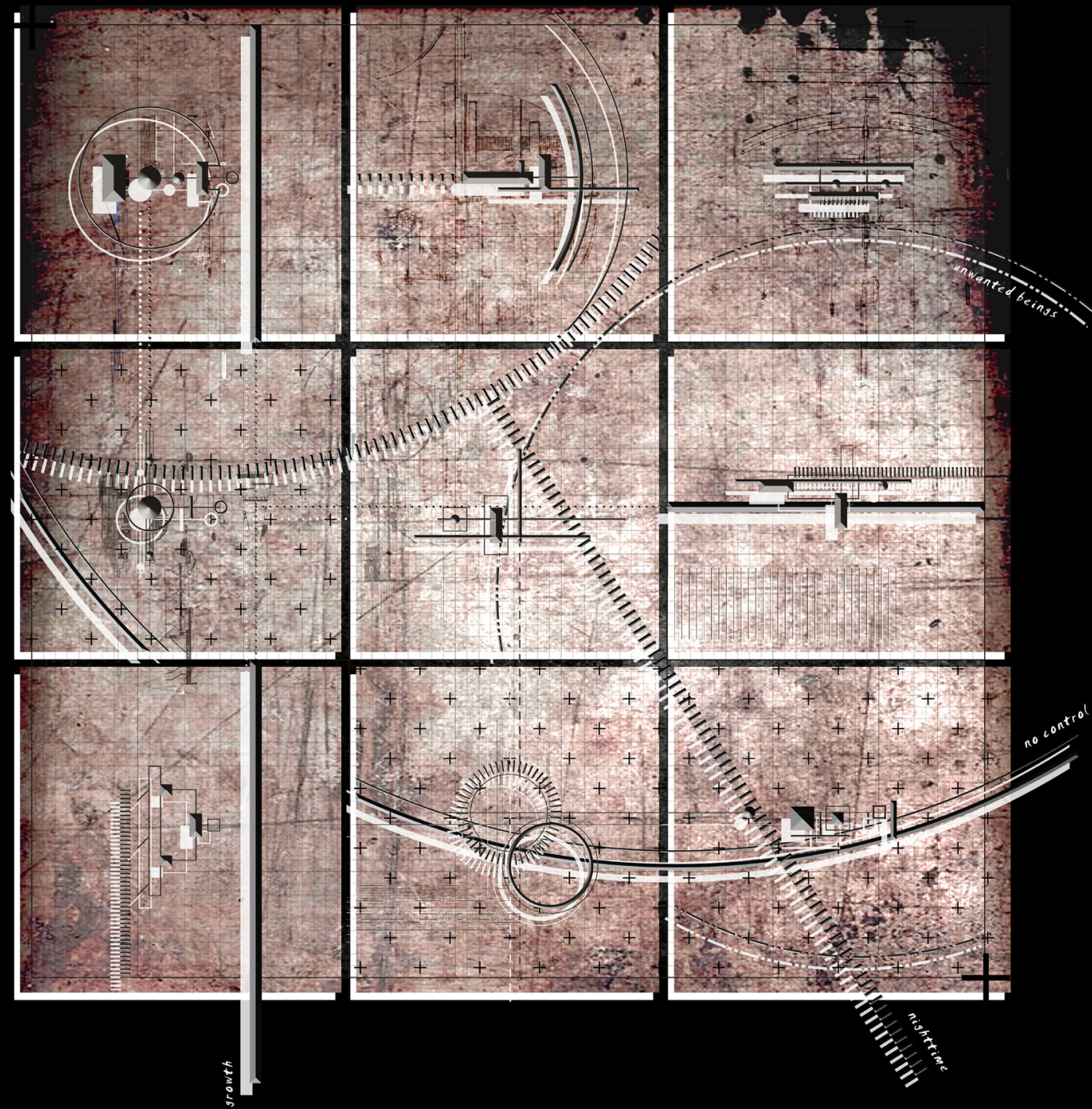

Fig. 50. Conceptual Notation Diagram Drawing Links between 9 Artefacts [\#2. Abstraction through Plan view] 
These two conceptual explorations (Fig. 51 and Fig. 52) tests through shadow-play how perspective and perception play a role in altering the concept of space and form. The overlapping relationships become altered through the shift of the camera/ eye. This introduces the notion of the individual's perception as requisite for constructing a personalised narrative architectural experience. These conceptual exploration begin to illustrate how the resulting forms from Design Stage 1 can be developed to achieve the Research Objectives set out in RO1-3.

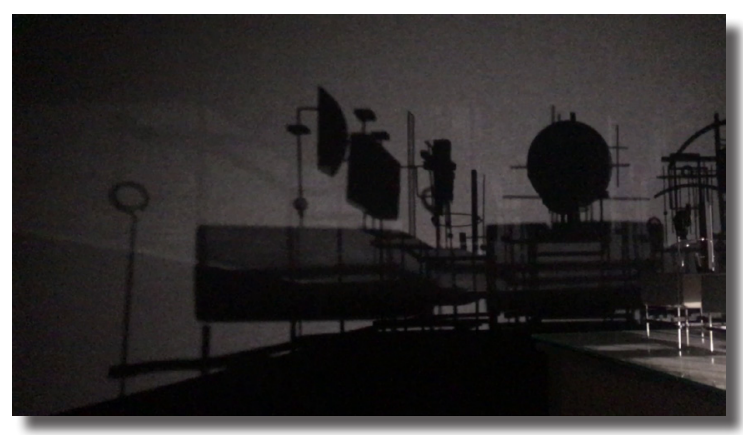

(D) Refer to video file

Fig. 51. Conceptual Exploration of Shadow-play

This video interrogation represents an exploration of a transition from the physical models of Design Stage 1 to the virtual game environment of Design Stage 3.
RO 1: To explore how culturally scripted ideas can be explored and expressed through allegorical methods in the conception of an evocative architectural framework that deviates from Eurocentric modernist principles and constructs.

RO 2: To explore how narrative architecture can activate allegory within the experiential as a tool for reinterpreting the notions of 'traditional' architectural elements through their shift in spatial conditions over time.
RO 3: To explore
how Digital Gaming Environments can be actively engaged in the architectural design methods and processes to enable agency to actively participate in the experiential narrative outcomes. 


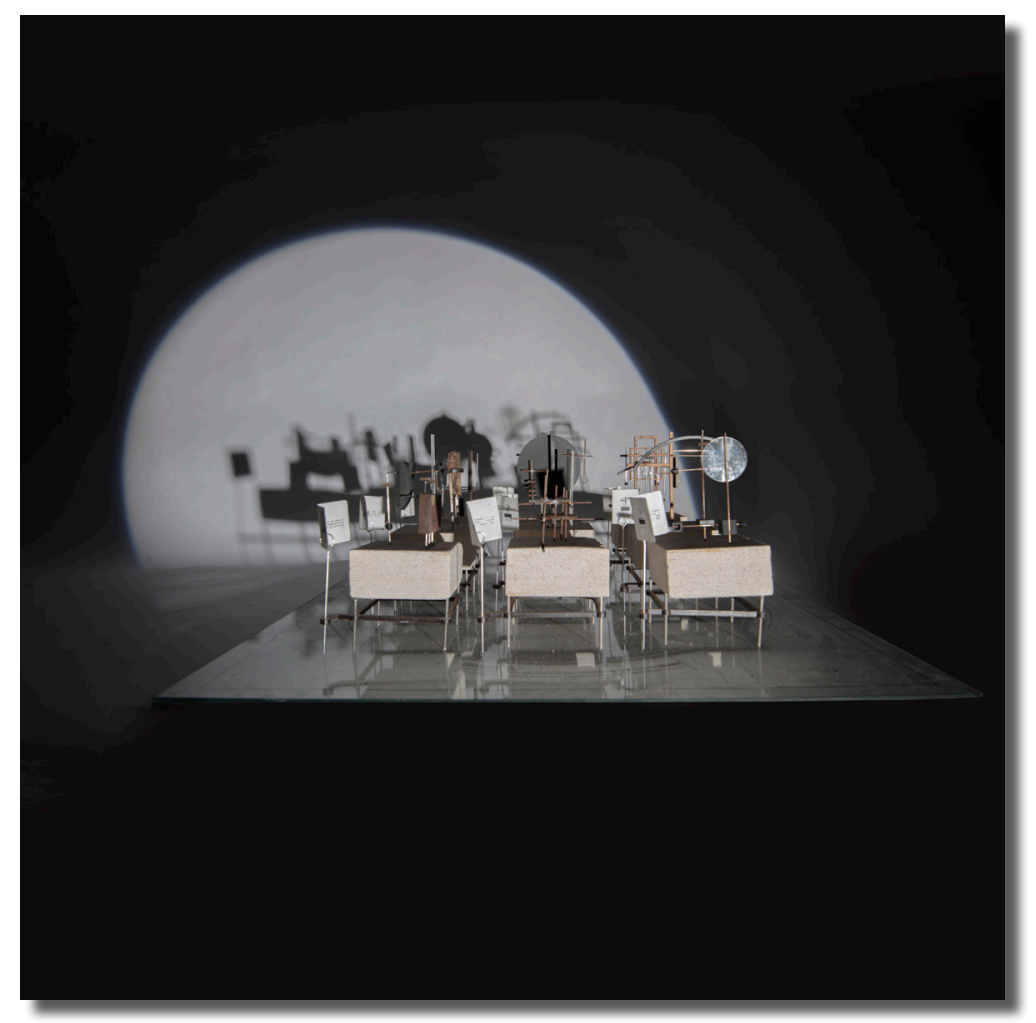

Fig. 52. Relationships of the 9 Model Artefacts with each other interrogated through overlaying and shadow play 

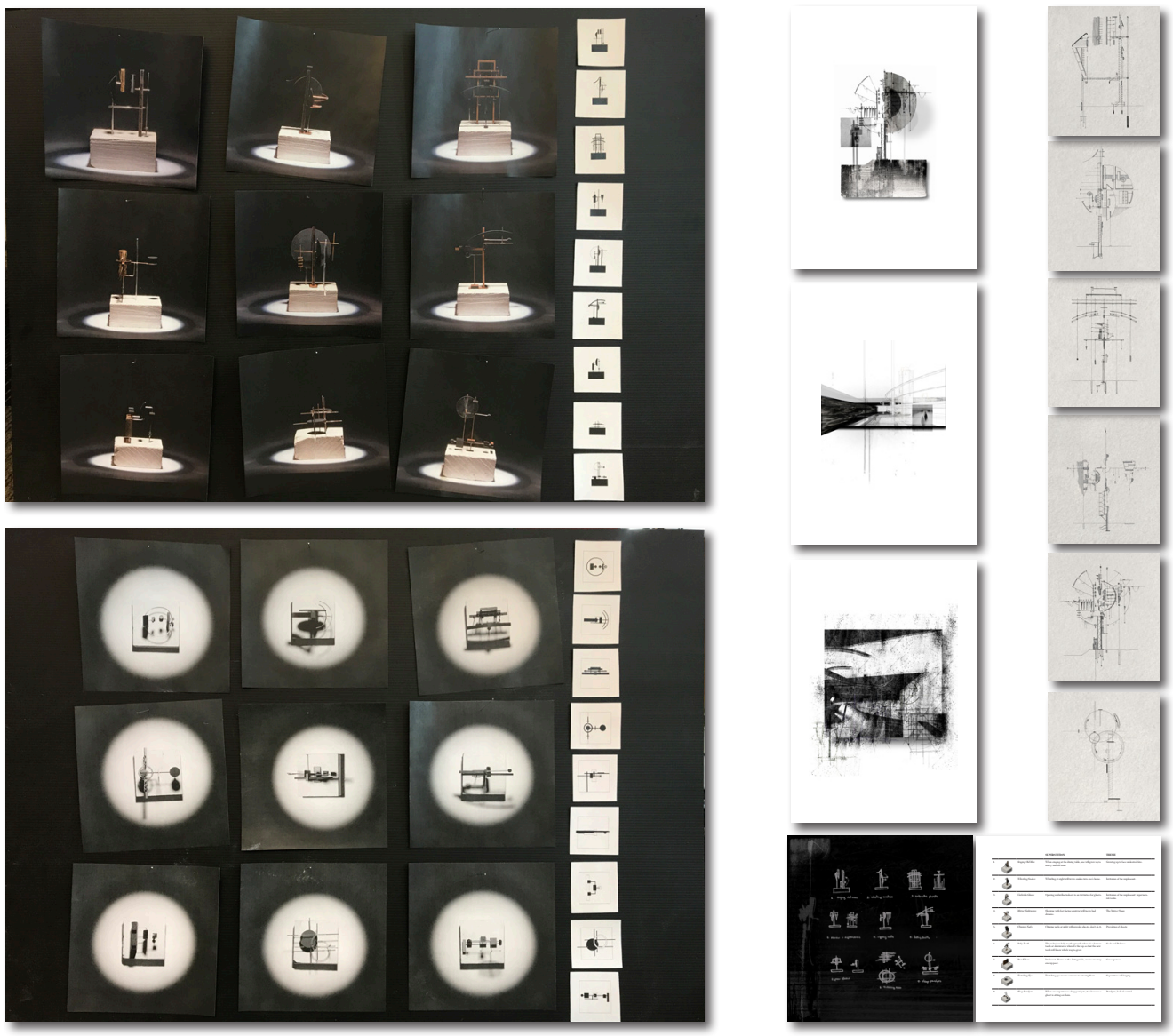

Fig. 53. Design Stage 1 Set of Drawings 


\section{Critical Reflection}

\section{REFLECTION ON DESIGN REVIEW 1: FEEDBACK AND DISCUSSIONS}

A formal Design Review for Design Stage 1 was held on 23 May 2019. The conversation centred around the degree of architecture being achieved and the privileging of spatial experience over form,

\section{- How is it architectural?}

Even though the formal outcomes from Design Stage 1 are not 'architecture', there are elements that represent architectural notions such as threshold conditions, verticality, gateways, circulation and moments of habitable spatial conditions.

These elements that imply architectural notions are developed further in the next two design stages, as the investigation advances from Figurative Geometries (Design Stage 1) to Progress and Journey (Design Stage 2), and ultimately to Invitation for Interpretation (Design Stage 3).

\section{- Spatial Experience over Form?}

The formal explorations achieved in this stage were treated as the basis for exploring spatial and temporal conditions as an architectural experience. 


\section{REFLECTION ON PRELIMINARY DESIGN: DESIGN STAGE 1}

The design explorations undertaken in Design Stage 1 set out to explore how culturally scripted ideas can be explored and expressed through allegorical methods in the conception of an evocative architectural framework that deviates from Eurocentric modernist principles and constructs [ $\left.\begin{array}{ll}\mathrm{RO} & \mathbf{1}\end{array}\right]$.

The formal outcomes of the artefacts conceived in Design Stage 1 are assessed against the theoretical foundation behind the generation of the artefacts as figurative geometries, drawn from French writer and critic Roland Barthes's five codes of semiotics:

1. The Hermeneutic Code

2. The Enigma/Proairetic Code

3. The Symbolic Code

4. The Cultural Code

5. The Semantic Code.
The formal outcomes of the artefacts represent unconventional notions of formalising and expressing culturally scripted ideas. By employing a method of allegorical abstraction and sculptural installation to achieve formal properties, the results help move beyond expected 'traditional' architectural elements-in response to the Hermeneutic code, the Enigma/Proairetic Code and the Semantic code. Examples of where this has been achieved are: threshold conditions and framing devices, to name a few.

Although it introduces the concepts, Design Stage 1 needs to be developed further in Stage 2 in order to meet the criteria of the Symbolic Code and the Cultural Code as they require the next two stages to fully comply with all five codes. 
The Symbolic Code calls for the organising of the set through the uniting of the 9 artefacts to form dialogues with one another. Design Stage 1 introduces the notion that they are organised within a grid scheme and their formal overlays have been explored briefly; however, the uniting of the set of 9 in the game environment will be the final point required to meet the $3 \mathrm{rd}$ code.

The Cultural Code calls for the removal of any overt cultural implications in order for the individual to apply their own personal perspective into the meaning and interpretation of space. The artefacts in Design Stage 1 use formal vocabulary that deviates from cultural/traditional lineage; but the application of the self within space is a concept that also requires the game environment of Design Stage 3 to have completely represented the Cultural Code.
Design Stage 1 is a design generating exploration exercise for the conception of an evocative architectural framework. The formal outcomes of the artefacts in Design Stage 1 are fixed notions as an object-based exploration; some of the weaknesses are that they lack the ability to convey dynamic movement due to this and they can only convey implications of the movement.

The next preliminary design stage (animated space) will help enable the actualisation of these concepts through their ability to convey notions of movement through time-based events as a tool to reinterpret architectural elementswall, floor, threshold, etc., setting out to achieve RO 2: To explore how narrative architecture can activate allegory within the experiential as a tool for reinterpreting the notions of 'traditional' architectural elements through their shift in spatial conditions over time.

Through the opportunities and abilities of the process in the next stages, the design series will be able to more fully represent all five semantic codes. 
曲 
3.2 DESIGN STAGE

Stage 2

INTO THE MOTION

Fxploring Shift in Spatial

Conditions through Animations

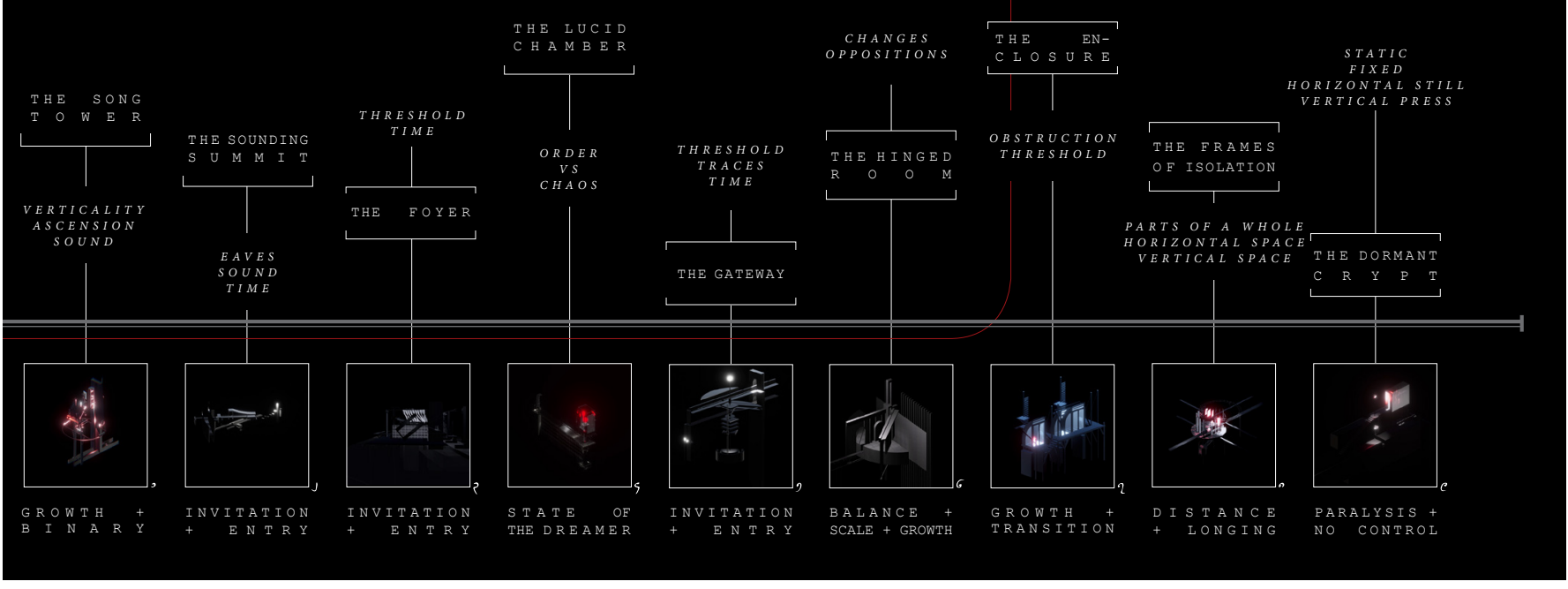




\section{Animated Space PROGRESS AND JOURNEY}

RO 2: To explore how narrative architecture can activate allegory within the experiential as a tool for reinterpreting the notions of 'traditional' architectural elements through their shift in spatial conditions over time.

the cinema incorporates time to space. Better, time,

through this, really becomes a dimension of space

- Faure 268 
$\mathrm{RO} 2$ of this investigation seeks ways to incorporate shifts in spatial conditions over time into the design of architecture.

Time is an important aspect of how we experience and understand architecture, but time is rarely invoked to explore and question spatial and experiential qualities and how they may change over a course of time as new events unfold.

Design Stage 2 employs the digital animation medium as a tool to interrogate how the conventional norms in architecture can be reinterpreted and challenged by observing the effects of changes in spatial conditions unfolding over time.

Time-based animation schemes are employed in this stage as a way to demonstrate Bruner's narrative component, diachronicitythe narrative construct of the understanding of events as they unfold over time.
The architectural notions are challenged against the notion of time and redefined to be understood as a temporal spaces bound by a sequence of events as Fletcher's notion of an experience of Progress and Journey.

The design investigation series in this stage introduces time as a factor that shifts the spatial conditions of the formal outcomes conceived through Design Stage 1.

9 design explorations are carried out through the animated schemes in Design Stage 2-each a development of the 9 artefacts from Design Stage 1. The design explorations interrogate how the formal outcomes of the 9 artefacts can be framed using their implications of architectural notions to redefine and reinterpret 'traditional' architectural elements-room, floor, wall, threshold, etc. within a time-bound medium. 


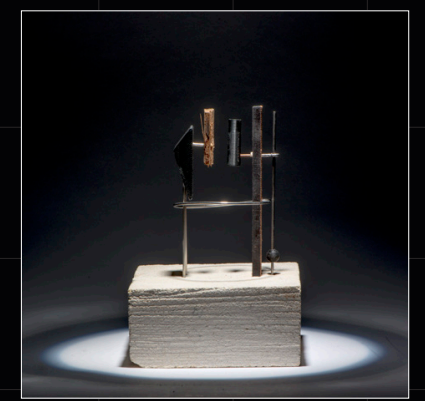

based on artefact 1 . Singing Old Man

- When singing while eating, one will grow up to marry an old man.

[Growth \& Binary]
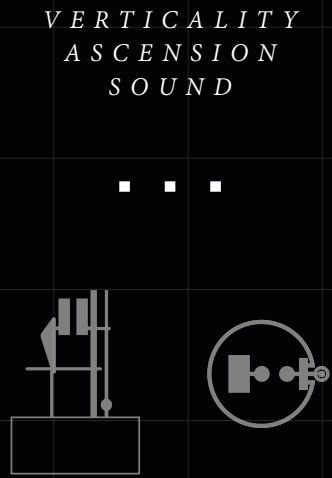

The Song Tower features two entities facing each otherone that is the 'young self' that shifts in vertical placement to come to face the second tower that is the 'old man'.
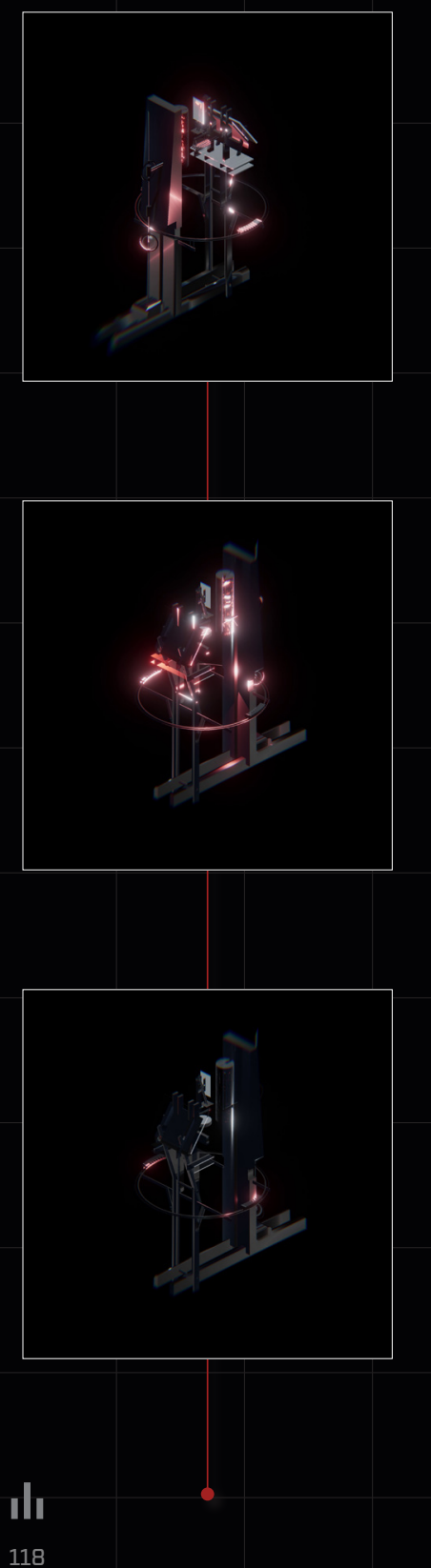

The formal construct of this exploration piece extracts directly from the model from Design Stage 1.

The tower of the 'young self' is depicted to be of simple form whereas the second 'old man' tower has a complex formal construct with many constituent elements.

This animated scheme presents itself similarly to a child's music box. The musical chime triggers the movement of elements. The first tower begins with its elements in vertical ascension and when the scene gets lit up, the piece begins to rotate, revealing the first tower from the perspective of the second.

The circular enveloping ring from the model that binds the two entities together is depicted as a railing system with tracks that house two elements running along the tracks.

As the musical chime slowly fades, the light that is now seen as emitted from the first tower begins to dim as well until the scene is no longer lit and sitting in silence. 


\subsubsection{The Song Tower}

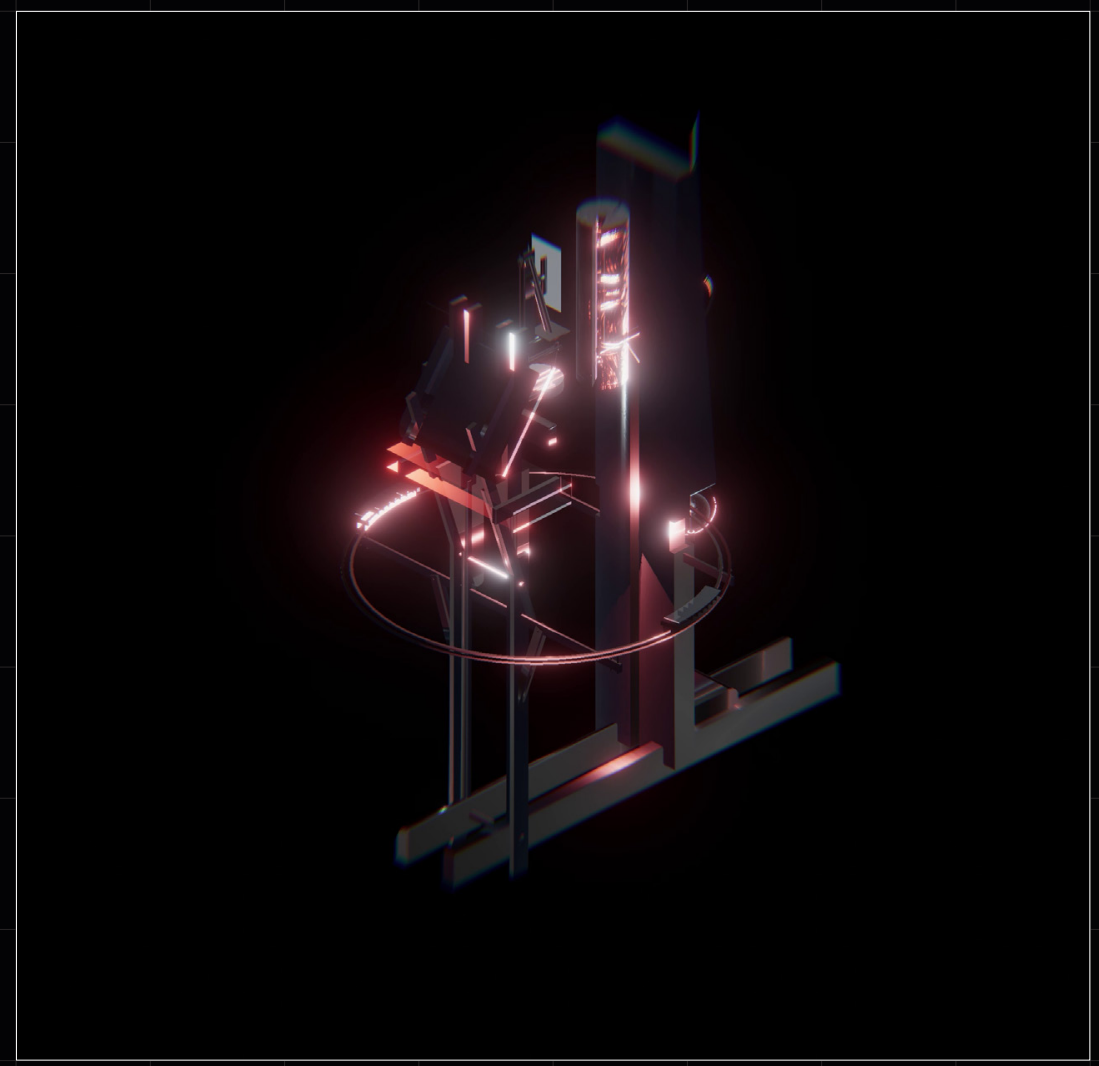

(D) Refer to video file 


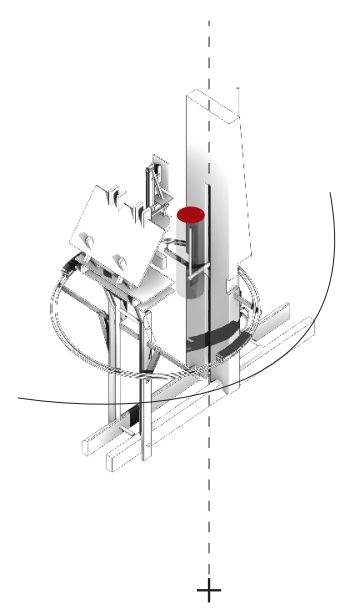

Fig. 54. Notation Diagram of Shift in Spatial Condition_\#1 The Song Tower Image by Author

The spatial notation diagrams illustrate movement of the events that unfold over time.

THE SONG TOWER interrogates the notion of duality through vertical ascension and orientation accompanied by light and sound. The two towers address duality through notions of similarity and difference. The differences compose their formal constructs while the similarities orient them to face each other. 


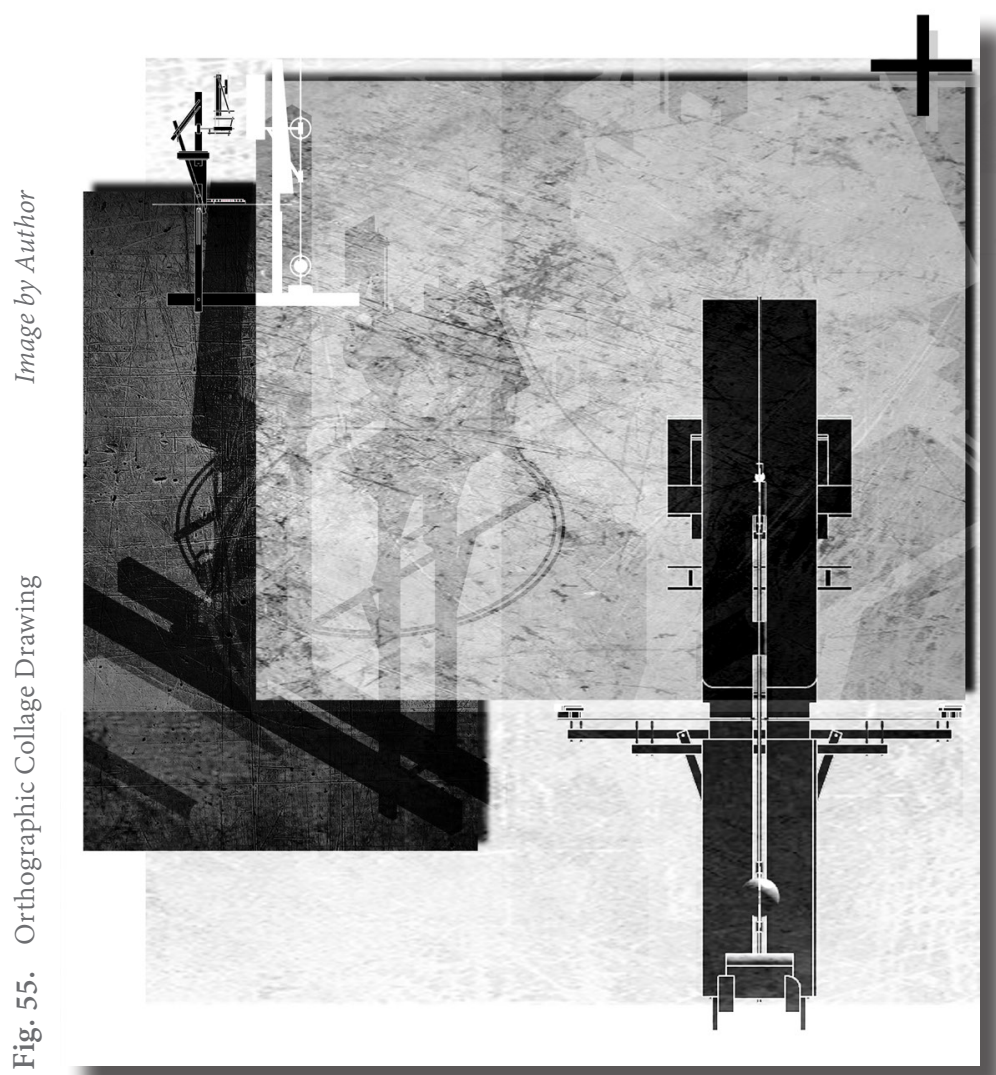

II 


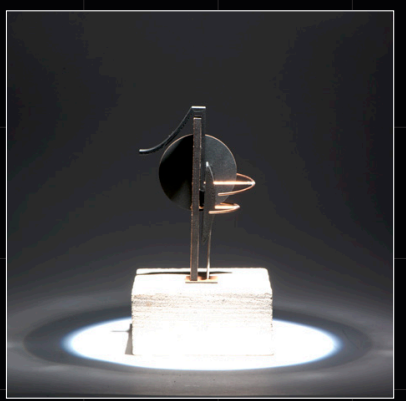

based on artefact 2 .

Whistling Snakes

- Whistling at night will invite snakes into one's home.

[Invitation \& Entry]

E A VES

$S O U N D$

TI ME
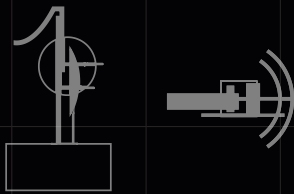

The conceived formal outcome of the whistling snakes artefact is developed in this animated stage to interrogate entry and invitation

[suggested

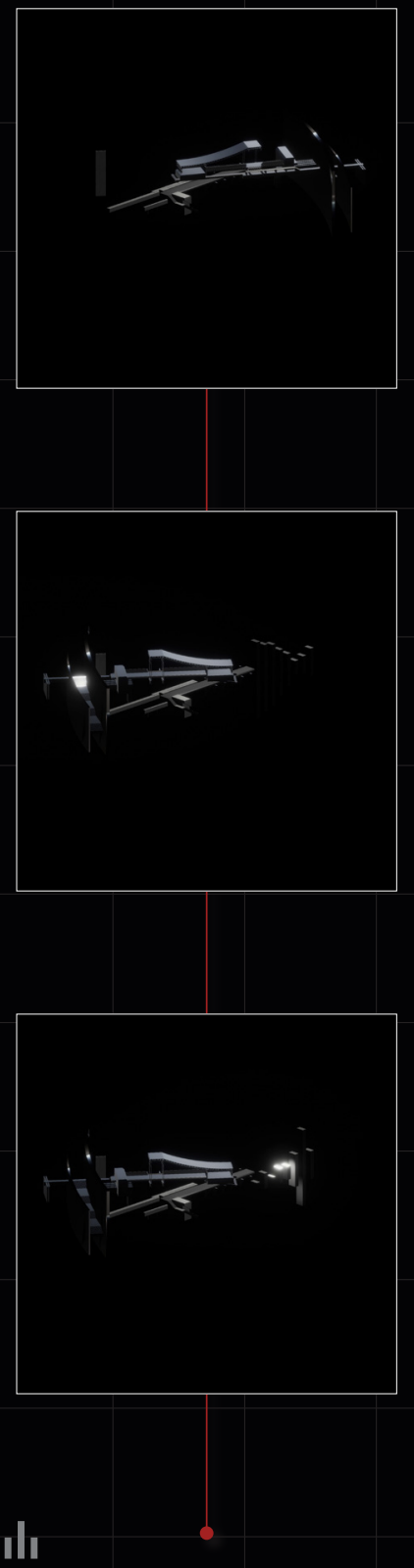

through the superstition's entry of snakes], through the notions of sound [the act of whistling] and time [the whistling at night].

The notion of the curved eaves are carried through to the formal developments in this stage where the main central roof form depicting the 'home' takes on the same form. The central piece pivots across the field depicting the notion of time.

The formal configuration of the branched out arc housing a staircase originates from the musical bars seen in the model depicting the notion of the whistling. The notion of sound is further interrogated through the shifting boxes that resemble music equalizer bars. Through their incline and decline, the staircase begins to reveal itself. The superstition that the whistling invites snakes is abstracted as sound being an invitation for entry that reveals the transitional element of a staircase. From the staircase, a light figure then enters through the central 'home' and exits the scene over the declining bars. 


\subsection{The Sounding Summit}

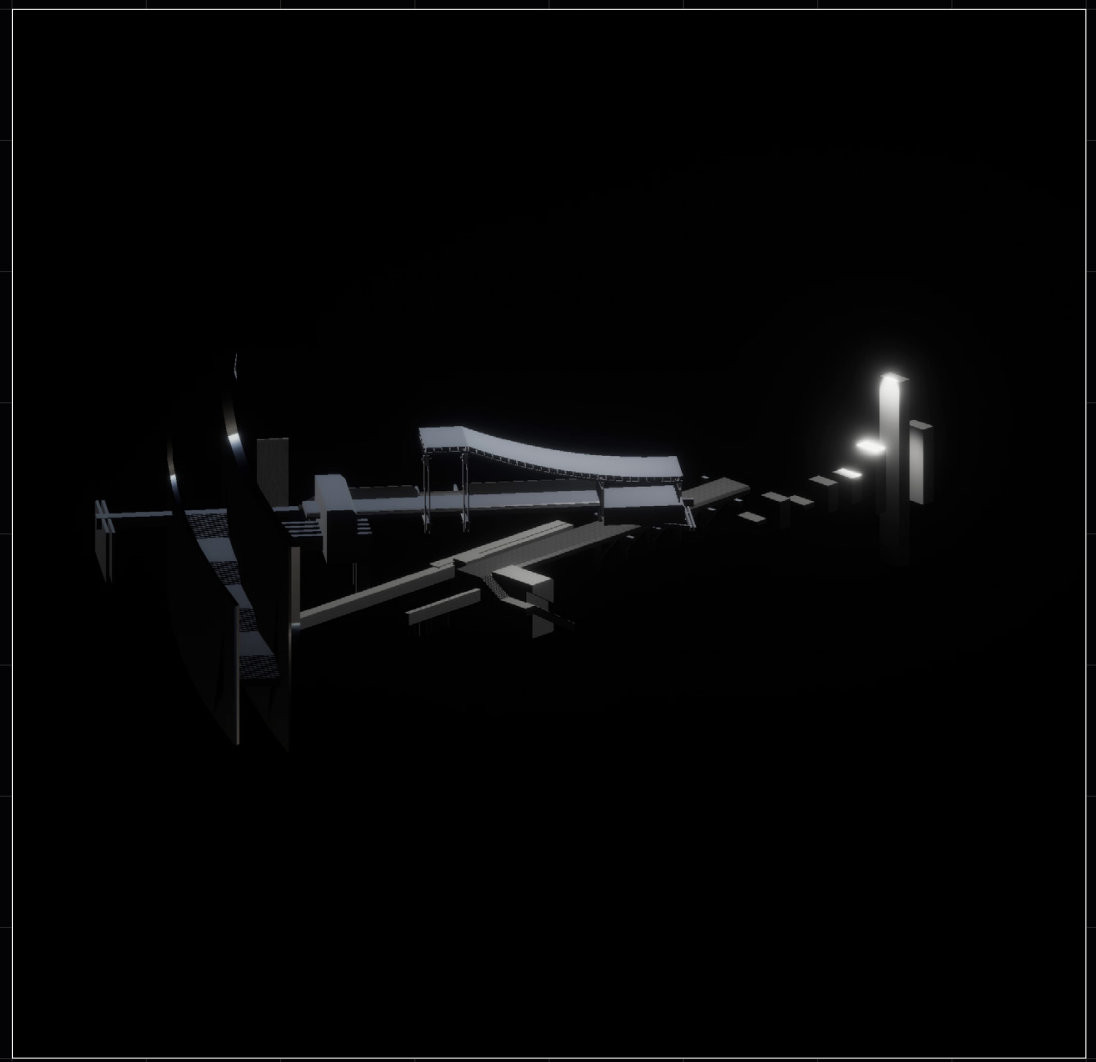

(D) Refer to video file 

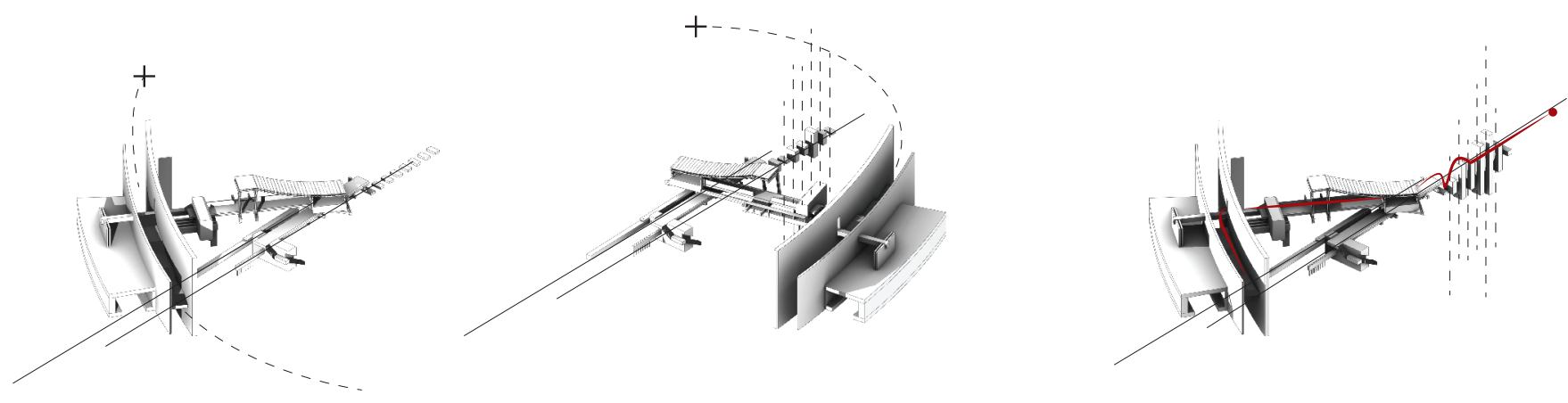

Fig. 56. Notation Diagram of Shift in Spatial Condition_\#2 The Sounding Summit

Image by Author

THE SOUNDING SUMMIT interrogates the notion of entry and threshold as a series of events occurring over time.

The roof element depicts a shelter-the setup of a threshold condition for transitioning into. The staircase conveys transitional notions anditdoes notexistatcertain pointsintime, until the 'musical bars' at the end start to shift in vertical scale. 


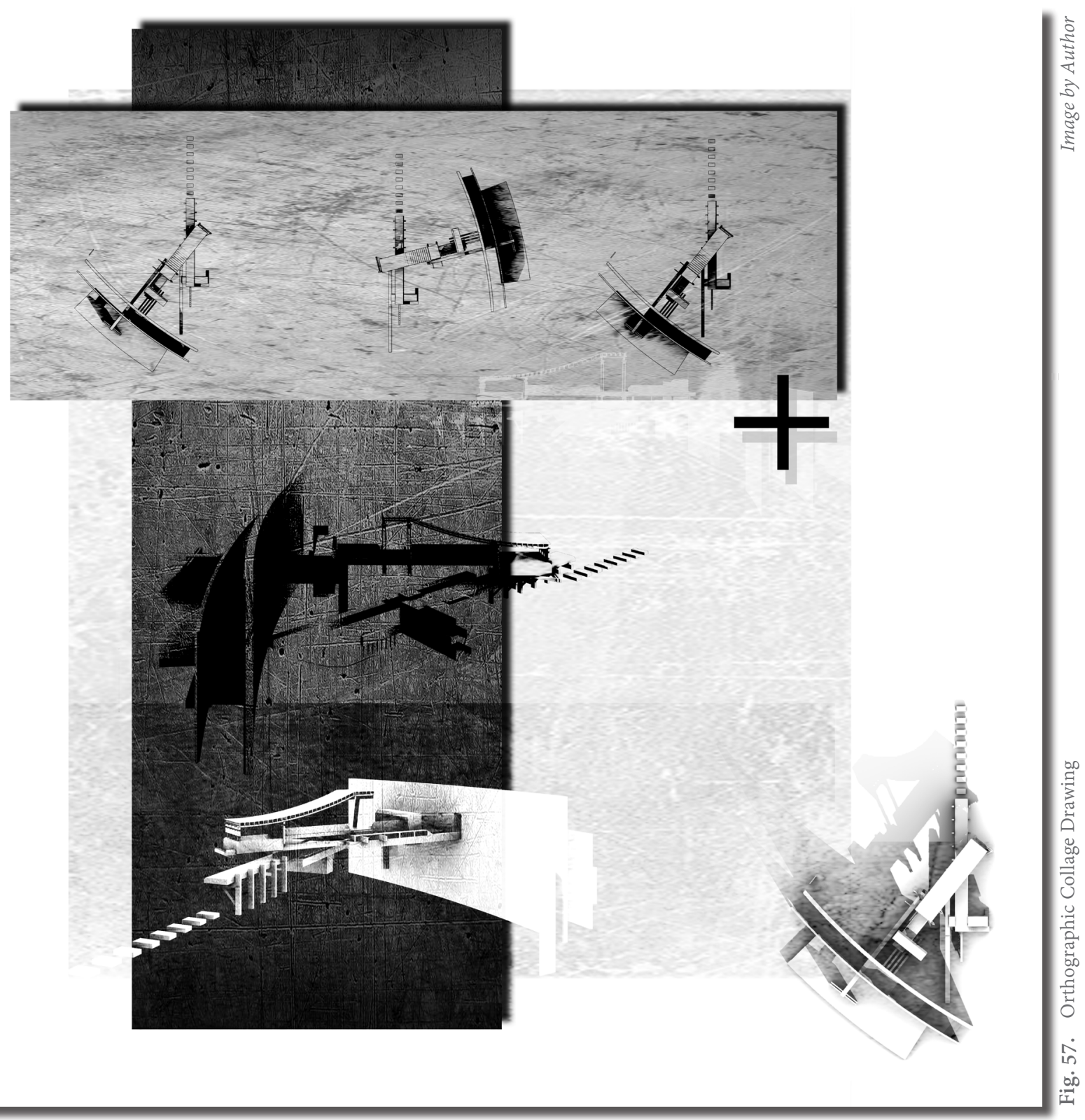




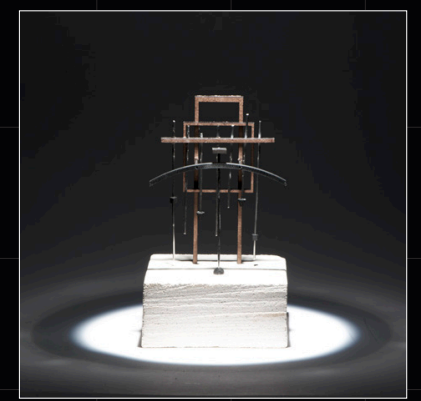

based on artefact 3.

Umbrella Ghosts

- Opening umbrellas indoors is an invitation for ghosts

[Invitation \& Entry]

THRESHOLD

T I ME
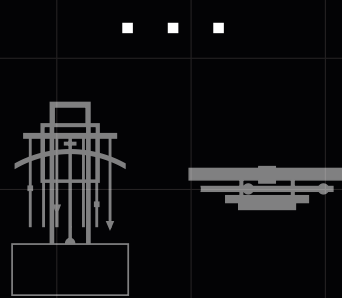
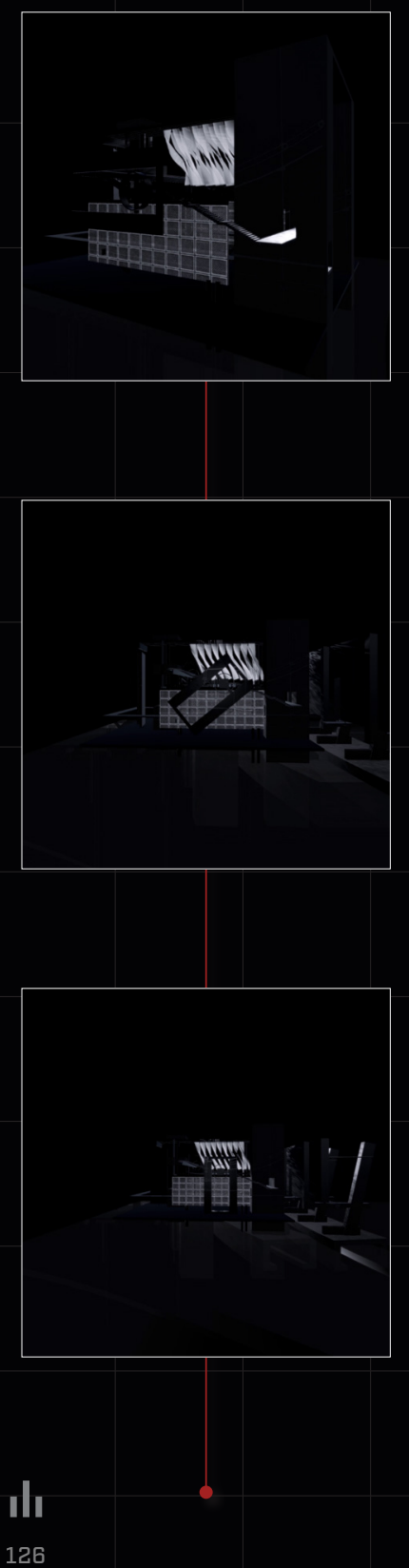

The notion of the umbrella opening within enclosed walls is developed further through this animation stage.

The external arc figure, taken from the physical model, moves out of the frame, representing the opening of the umbrella.

Two rectangular frames rotate around their circular pivoting axis, depicting the concept of time-moving through night and day.

The camera moves through the apertures and the rotating frame mimicking the entry of ghosts through the apertures.

The internal space consists of a dense layer of curtainlike translucent fabric, swaying gently in the wind.

The curtain configuration takes the formal properties of the physical model as seen in elevation as well as mimics properties of wind, and its translucency conveys a ghostly appearance. 


\section{${ }_{32.3}$ The Foyer}

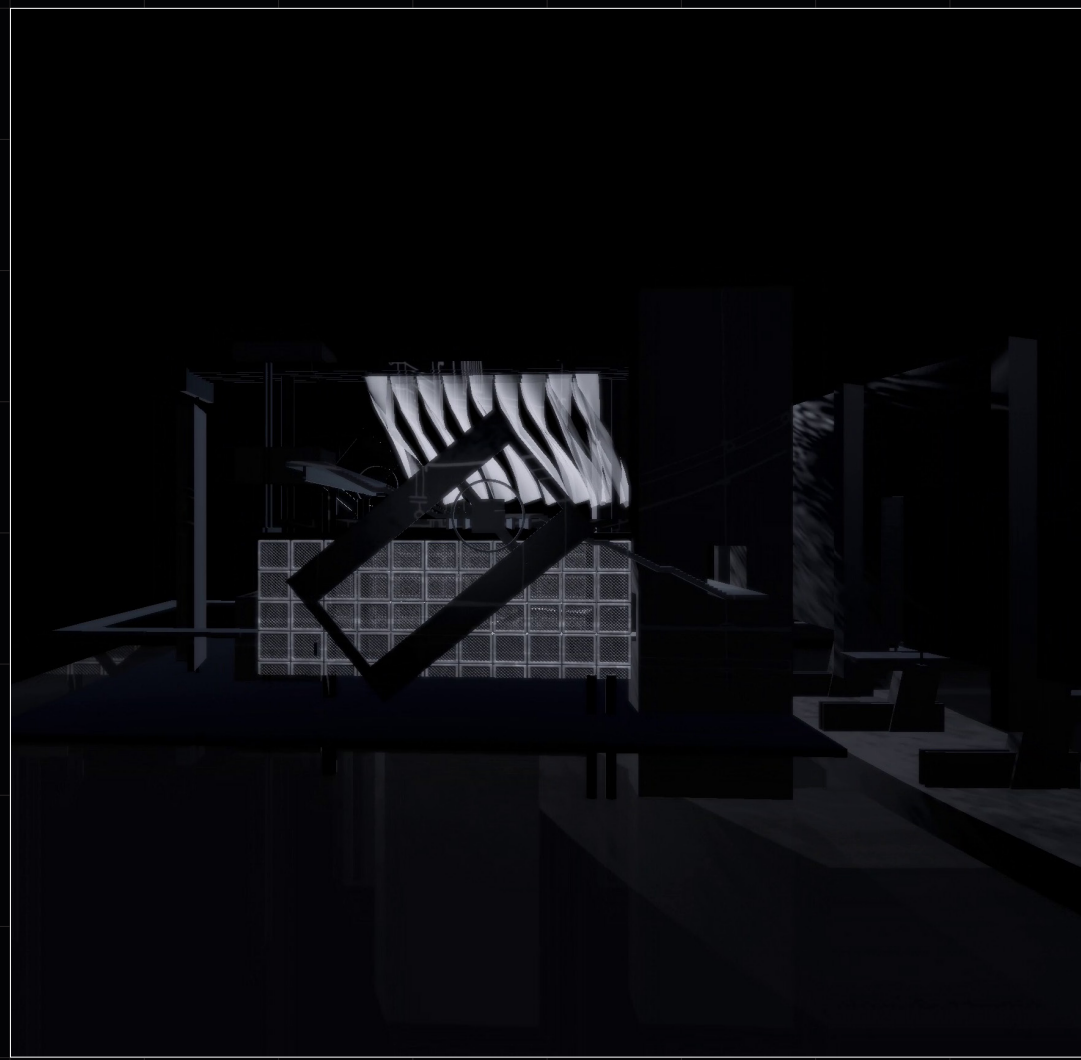

(1) Refer to video file 


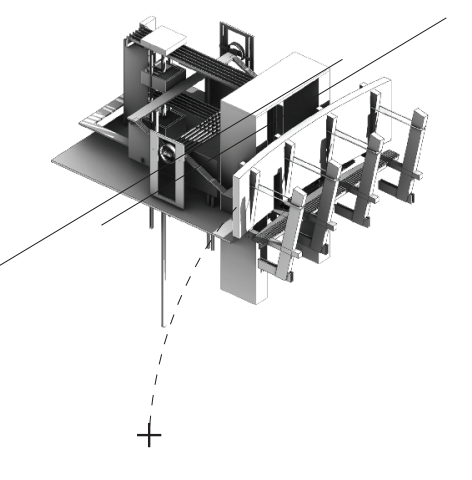

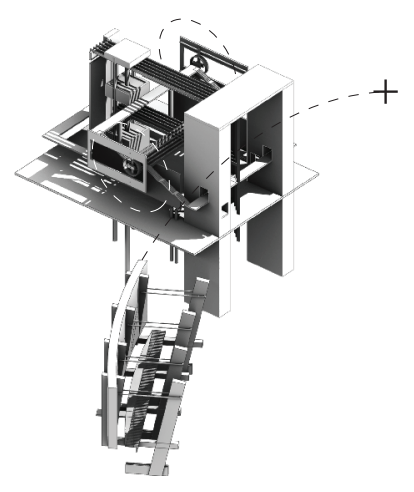

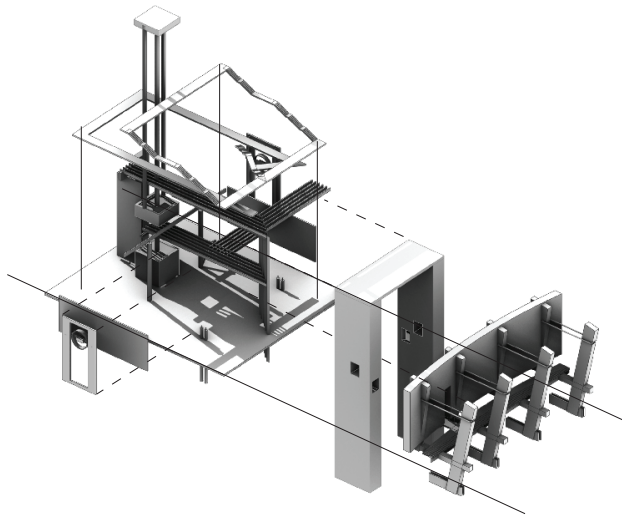

Fig. 58. Notation Diagram of Shift in Spatial Condition_\#3 The Foyer

Image by Author

This animated scheme, THE FOYER, also interrogates the notion of entry and threshold, and it has been associated with the spatial concept of a foyer-a transitional zone.

The notion of a foyer is the architectural interpretation of a space for passing through-a threshold condition composed through transitional elements such as apertures, stairs, gateway and framing devices. 


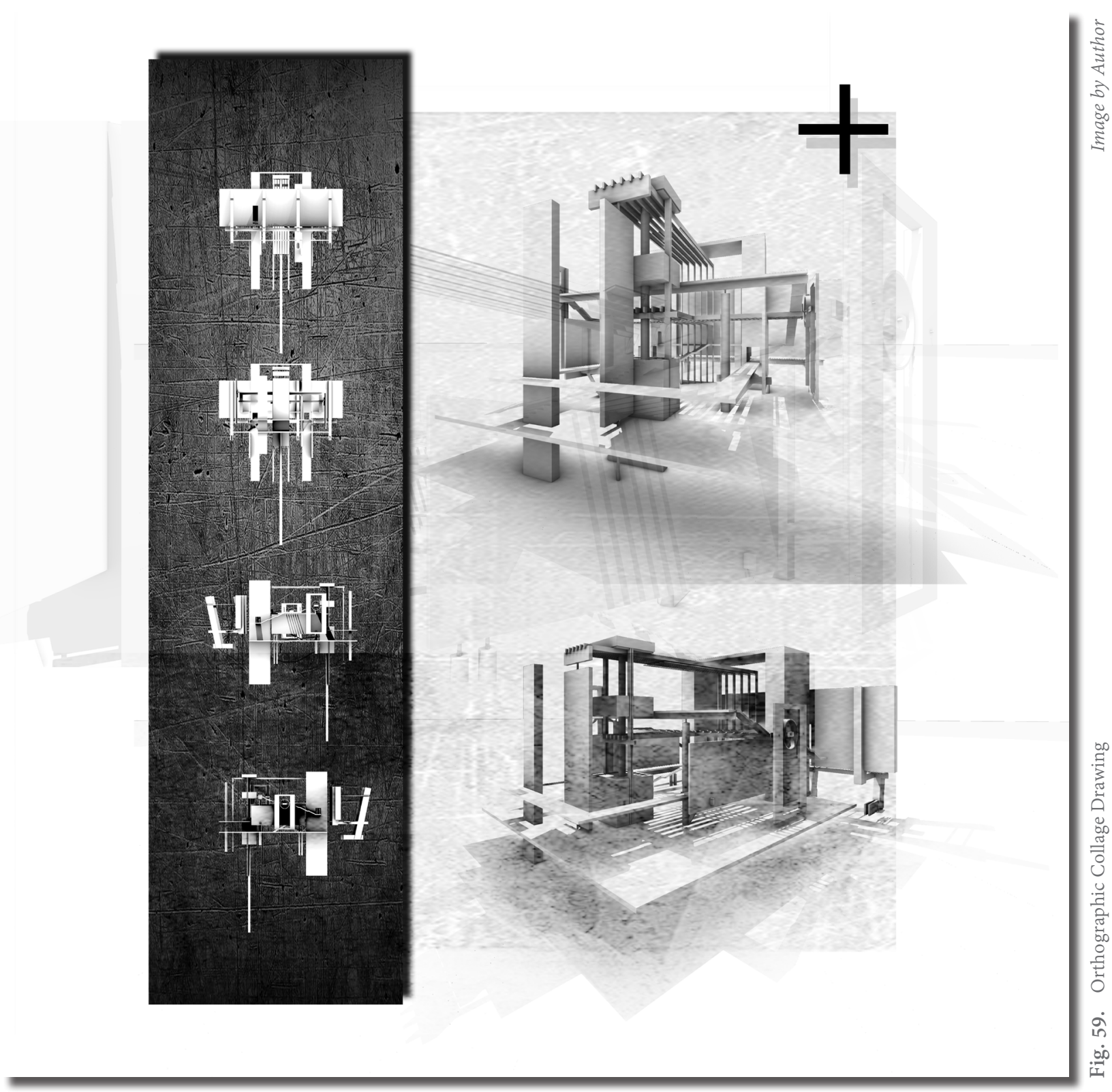




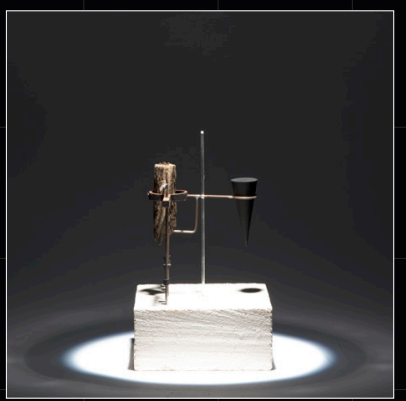

based on artefact 4.

Mirror Nightmares

- Sleeping with feet facing a mirror will incite bad dreams.

[State of the dreamer]

$$
\begin{gathered}
\text { ORDER } \\
V S \\
C H A O S
\end{gathered}
$$
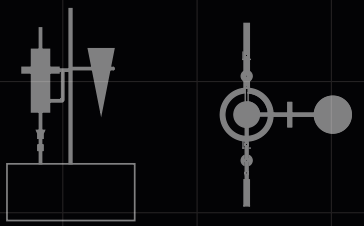
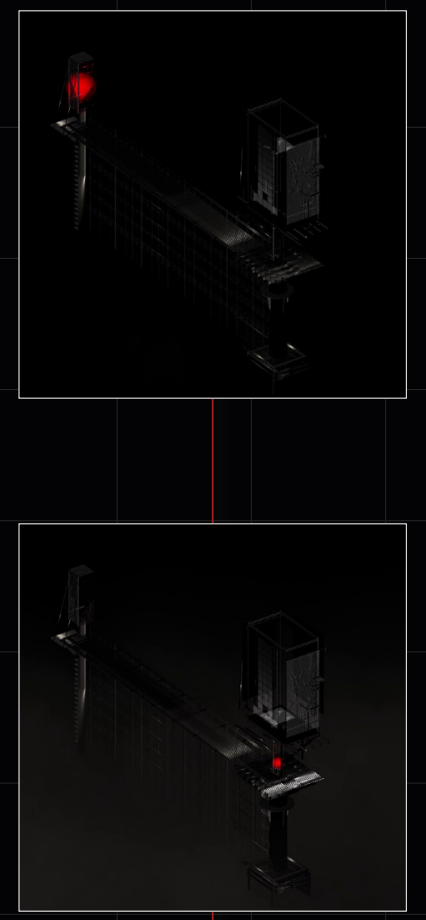

This piece uses the movement of a red light to depict the stages of sleep of the dreamer.

From the first tower, the dreamer falls into slumber, then shifts towards the second tower where the dreamer enters the REM state.

The red light transforms from a simple orb into a complex and chaotic light projecting onto the encased walls. The projected red light comprises previous drawing investigations from Design Stage 1. The red light becomes a projection of the nightmare realm.

From here, the light returns to its orb state, exits the tower through the central core and out of the frame-exiting the dream. 


\section{${ }_{324}$ The Lucid Chomber}

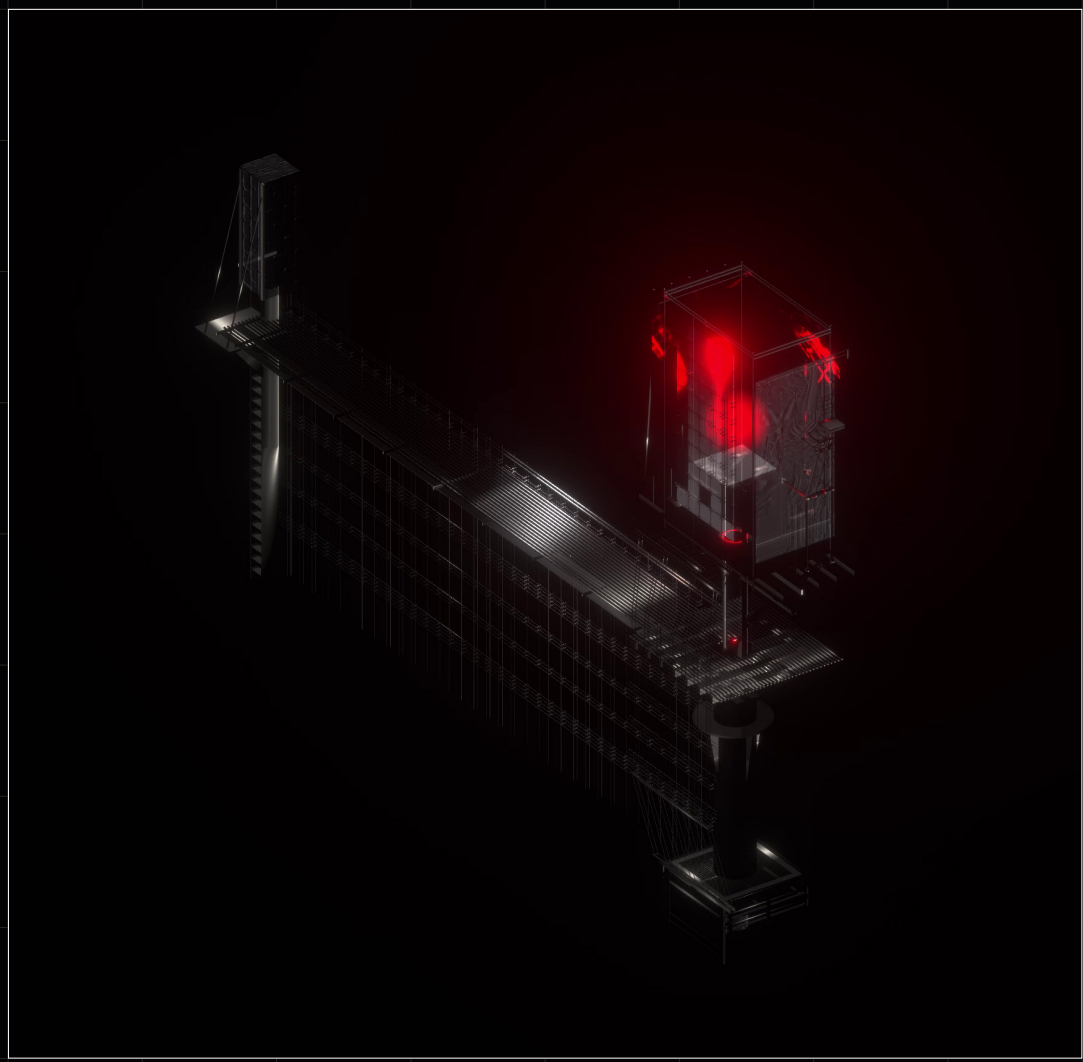

( $)$ Refer to video file 

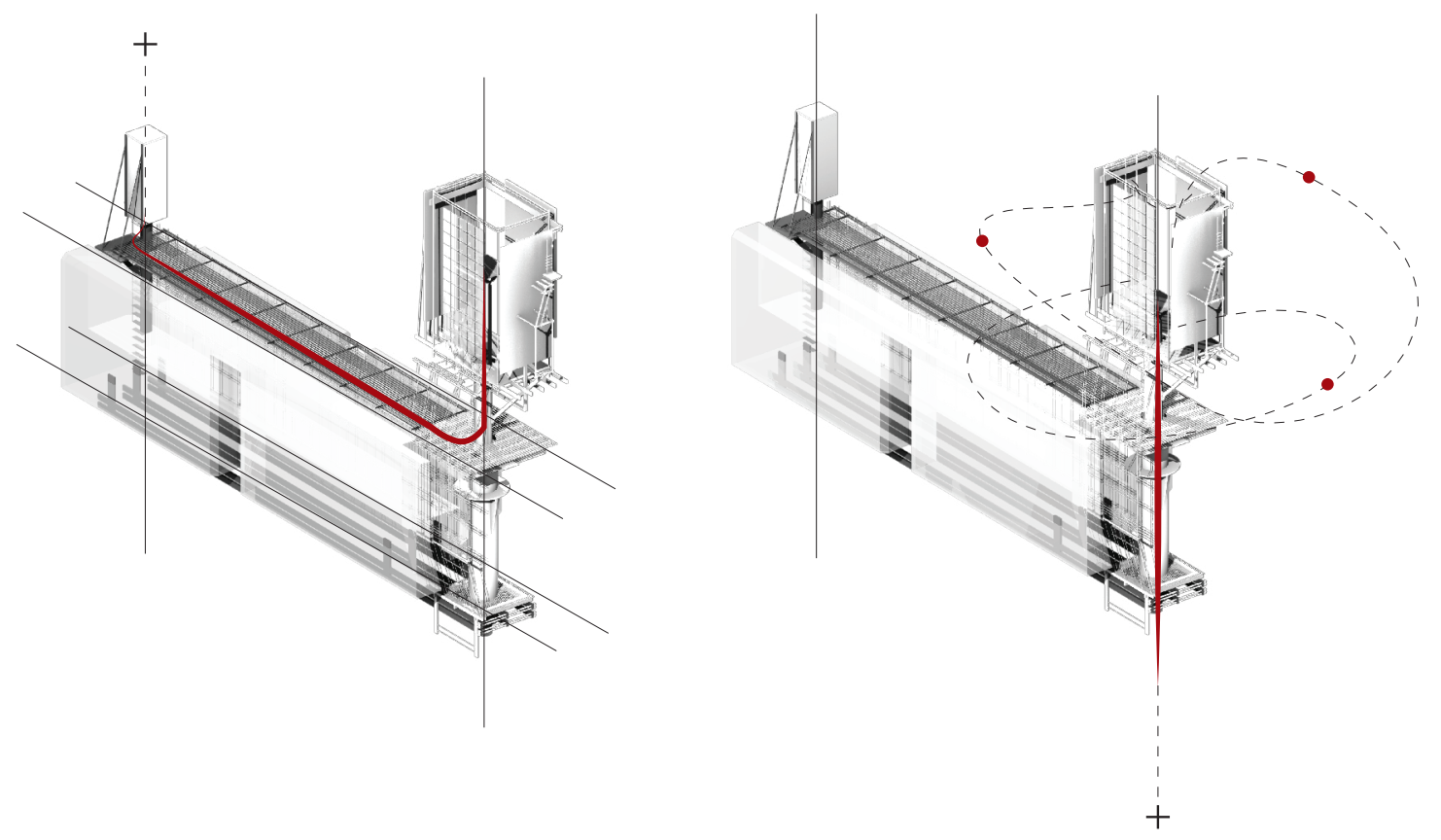

Fig. 60. Notation Diagram of Shift in Spatial Condition_\#4 The Lucid Chamber

THE LUCID CHAMBER interrogates the duality of order versus chaos through the behaviour of light and the formal construct of two architectural tower entities. The light's controlled behaviour within the smaller and simpler space suggests a calm and tranquil ambience while the light's turbulent behaviour as projections within a larger and more complex space suggests a more vivid and lively atmosphere. 

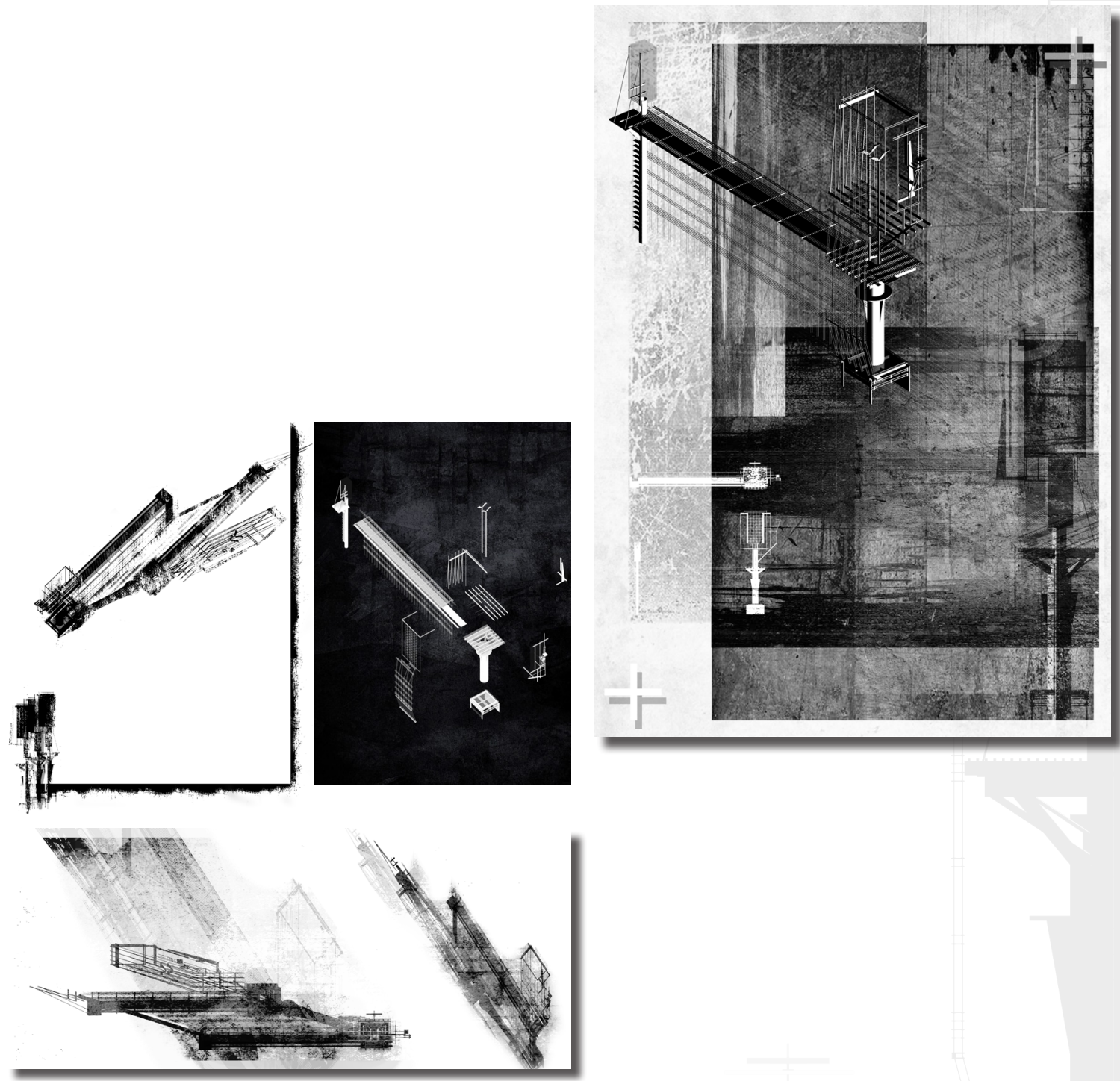

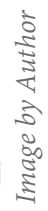




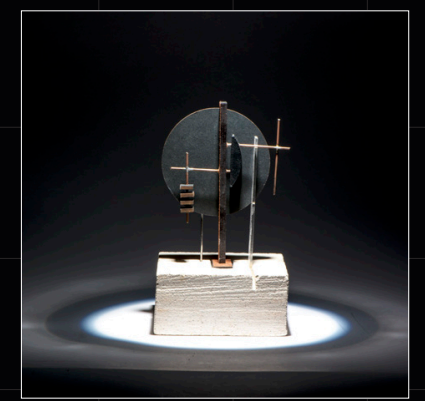

based on artefact 5.

Fingernail Ghosts

- Clipping nails at night will provoke ghosts.

\section{[Invitation and Entry]}
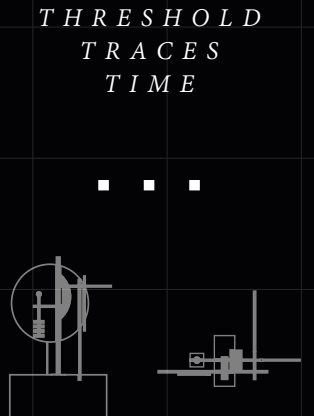

The notion of time [the clipping of nails at night], threshold [entry of ghosts into the home] and traces [carved out base] are explored in this animated scheme.
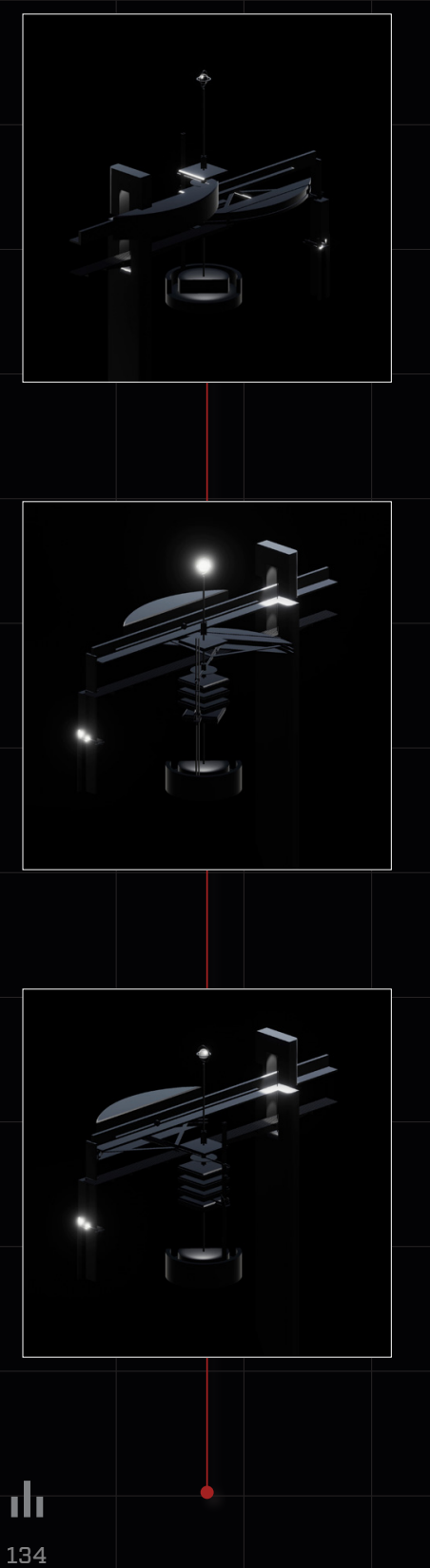

The notion of time is depicted through a rotational arc piece that pivots from the central pole. As the first oscillation comes to an end, a sliding entity begins to pass through to the end frame where it slips through an opening that fits its profile perfectly. The end frame with a spotlight indicates the notion of a threshold-a gateway. This scheme suggests transitional threshold conditions through reinforcing the concept of a carved out remain from an entity passing through a gateway.

The base of the central piece also conveys a carved out trace caused by the rotation of the time arc piece.

In the next frame the entire piece rotates around the central pole revealing the constituent parts of the central axis. The parts consist of 5 square sheets portraying the concept of 5 fingernails. One rotates with the rotational time arc piececonveying the notion that the action of clipping nails and nighttime are linked. 


\subsubsection{The Gateway}

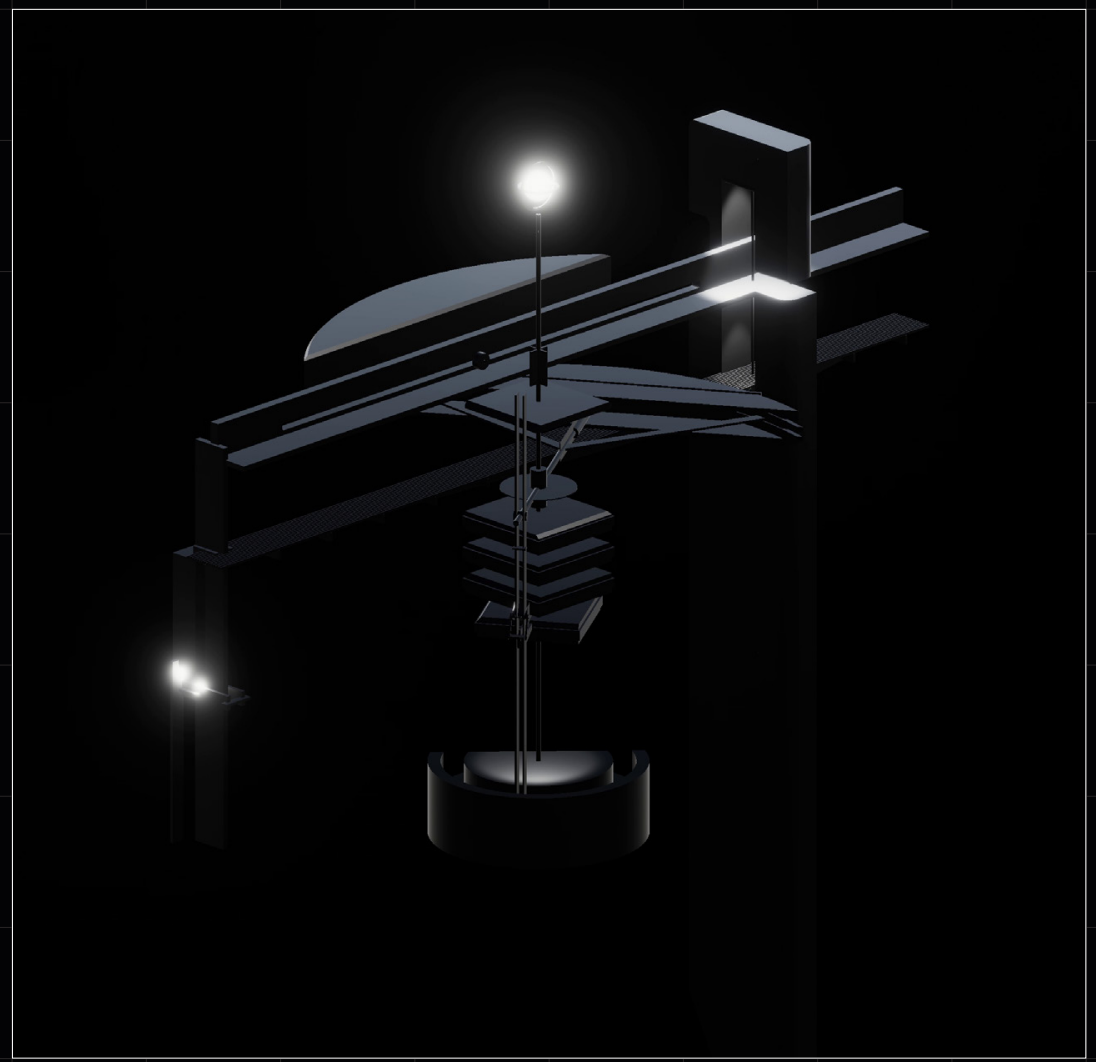

(D) Refer to video file 

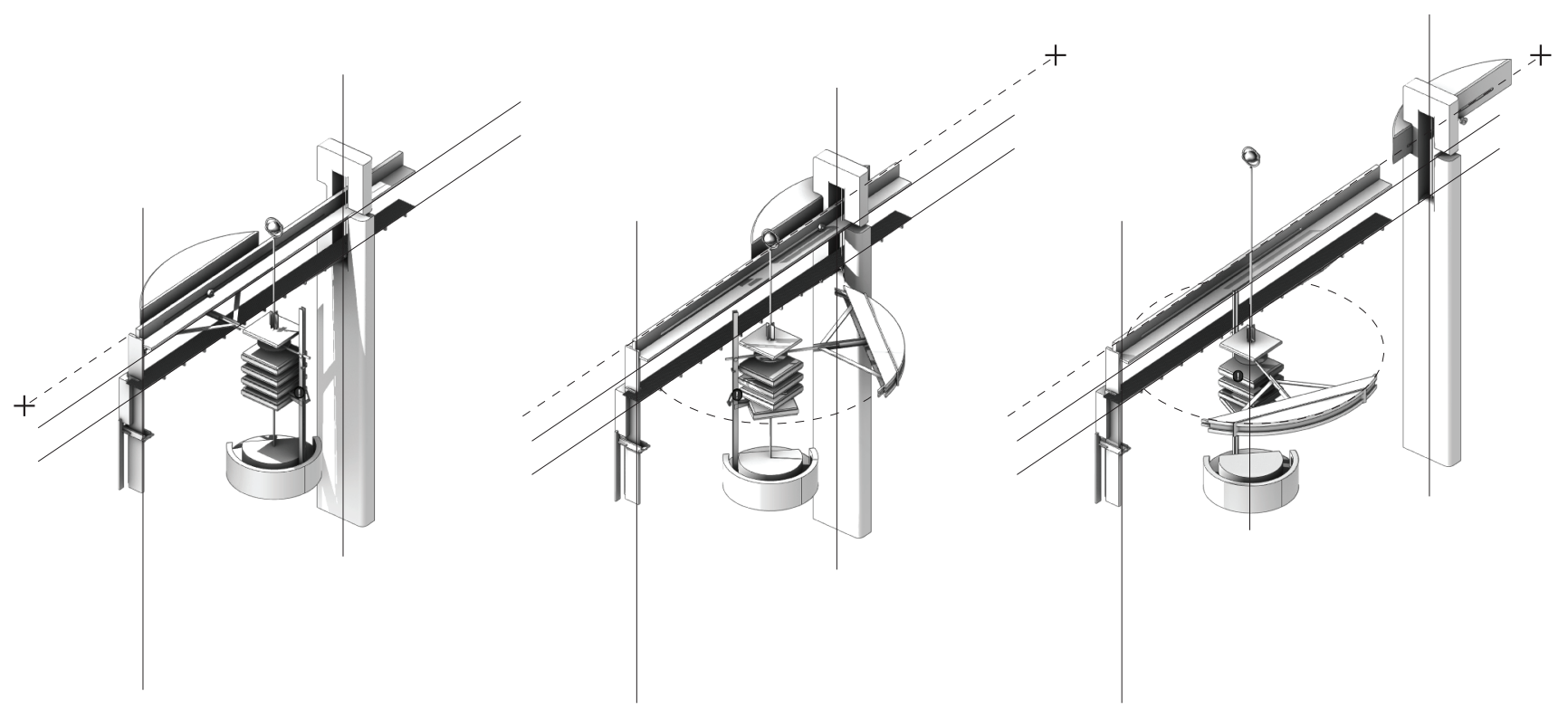

Fig. 62. Notation Diagram of Shift in Spatial Condition_\#5 The Gateway

Image by Author

This animated scheme of THE GATEWAY explores the notion of threshold. The end frame acts as a point of transition through which an arc figure slices into an opening slot, perfectly fitting through it. This act is triggered by the rotational pivot of the central piece-the time bound act of clipping at night triggers the event of entering.

This scheme gives opportunities of trigger events that could be implemented in the next stage within a game environment . 


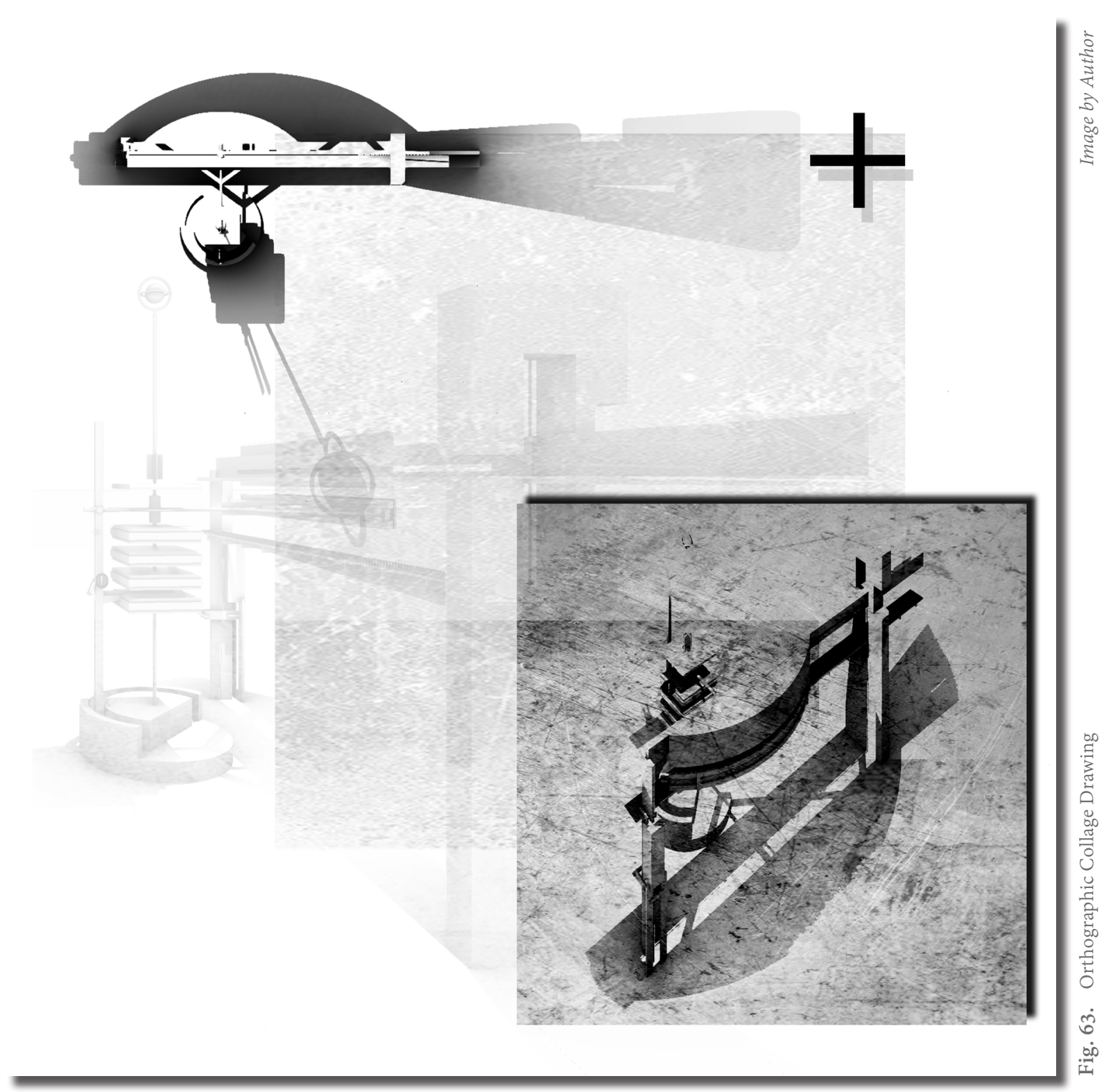

Ilı 


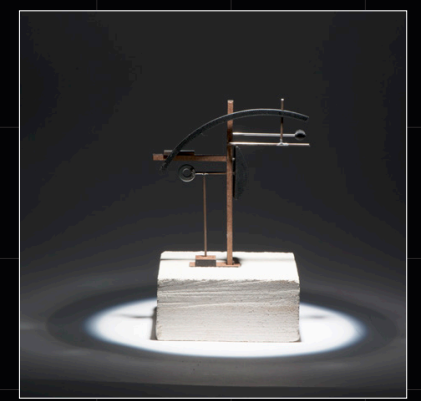

based on artefact 6 . Baby Tooth

- Throw broken baby tooth upwards when it's a bottom tooth or downwards when it's the top so that the new teeth will know which way to grow.

[Scale, Balance \& Growth]

$C H A N G E S$

OP POSITIONS

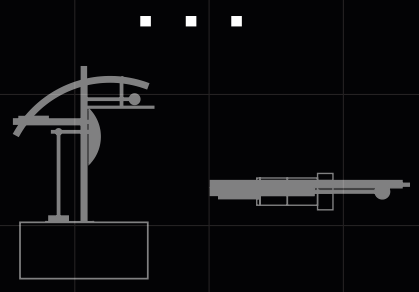

The concept of the hinge-a pivoting entity within architecture is used to develop the notion of scale and balance to challenge
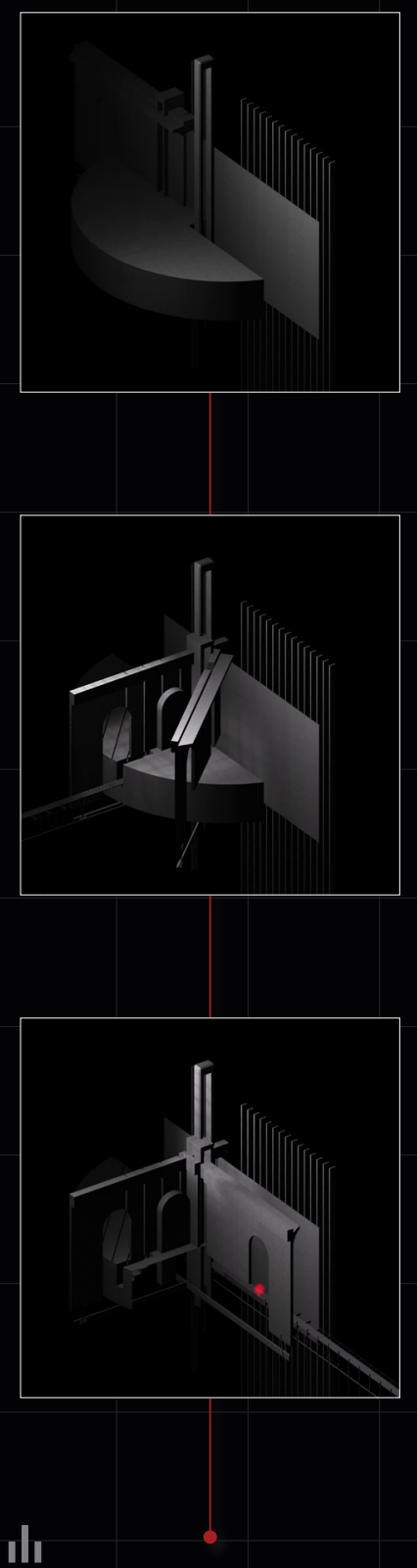

how spatial configuration can 'grow' and change over time to reveal a newly constructed space.

The changes and emerging of new spatial configurations refer back to the growth of a new baby tooth.

The concept here explores multiple elements pivoting along a central axis. The rotating elements open up to reveal a more complex spatial configurationwhere the internal elements are also exposed. This symbolises the complexities of life that inevitably come with growth into adulthood and the discovery of the internal self along the way.

The door opening symbolises new beginnings that lead to the extension of a platform, where the red light orb appears once again and follows the path exiting the frame.

The extended platform represents the new opportunities that arise through personal growth and the orb is the self seizing the new opportunities of life and embarking on a new journey. 


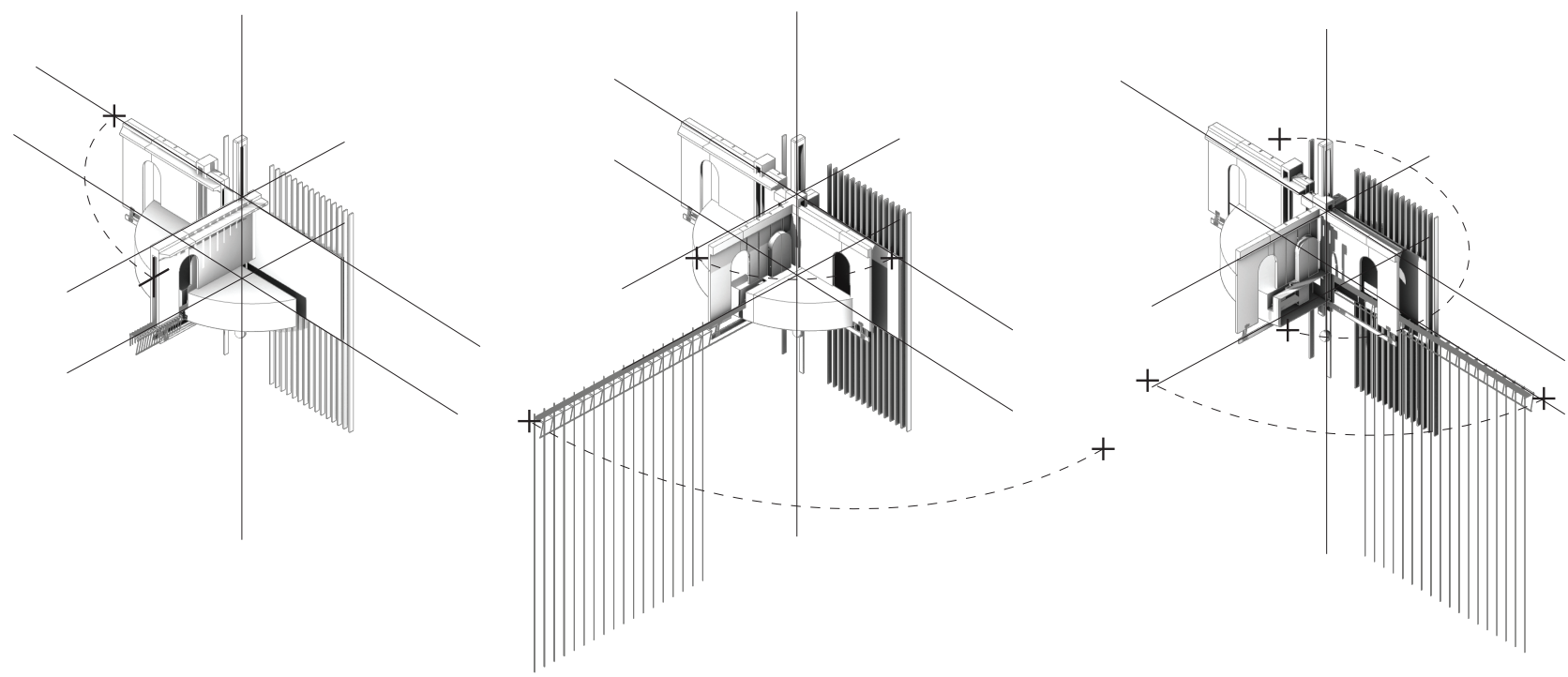

Fig. 64. Notation Diagram of Shift in Spatial Condition_\#6 The Hinged Room

Image by Author

THE HINGED ROOM explores the notion of the shift in spatial conditions through the revelation and opening of surfaces and "walls". The entire piece shifts the walls and floors through a central pivot point, where through their rotations, the condition of the space itself becomes transformed.

Notions of threshold and transitional elements are also present where it can aid the navigation of the player through the interventions in Design Stage 3. 


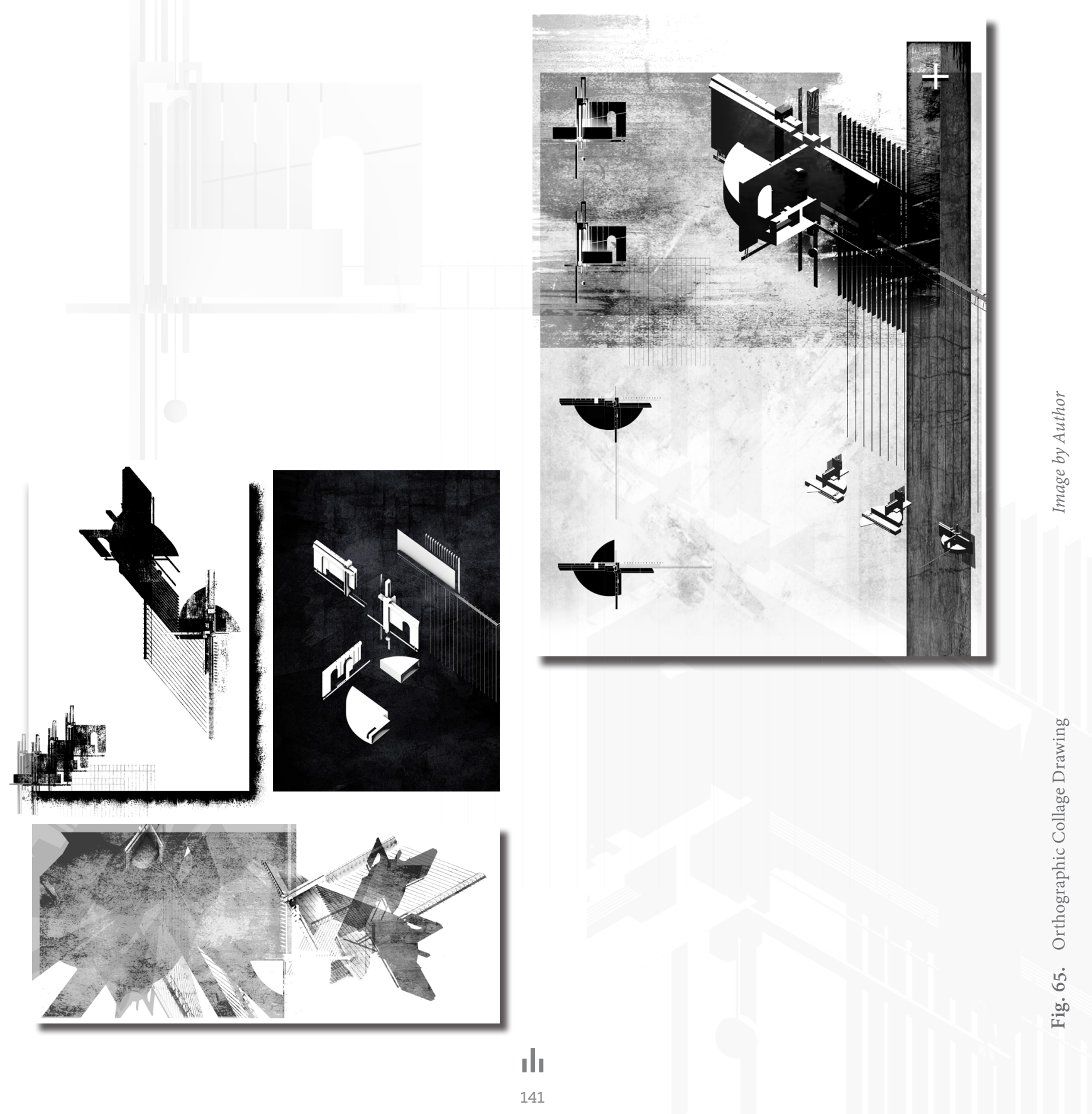




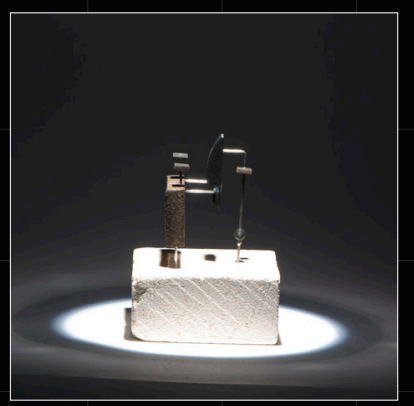

based on artefact 7.

Elbows of Misfortune

- One will grow up to face misfortune when placing elbows on the dining table.

[Transition \& Growth]

OBSTRUCTION

THRESHOLD

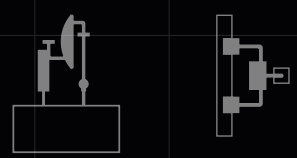

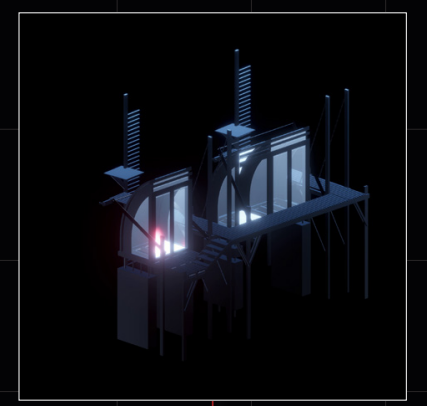

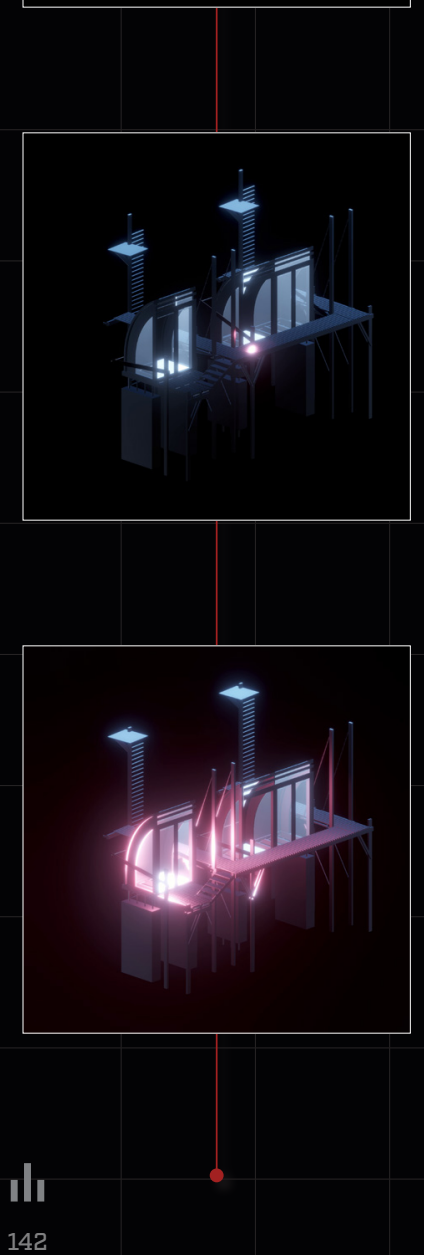

This animated exploration piece questions the notion of moving through spaces.

The concepts are based on the superstition that situating elbows on a dining table will lead to a life of misfortune. This is reinterpreted in spatial terms as not being able to transition to a desired point due to obstructions.

The red light acts as the occupant in this scene that interrogates this question. It attempts to navigate through the intervention piece; however the doors are always enclosing the light as it makes its way around into the two vessels. It depicts the obstacles that the light faces and is unable to freely transition to a desired point.

The light overcomes this obstacle by expanding its range so that it encompasses the entire surface of the intervention piece. Regardless of the containment of the enclosure, the internal conditions are able to permeate to the external. 


\section{${ }_{3.27}$ The Enclosure}

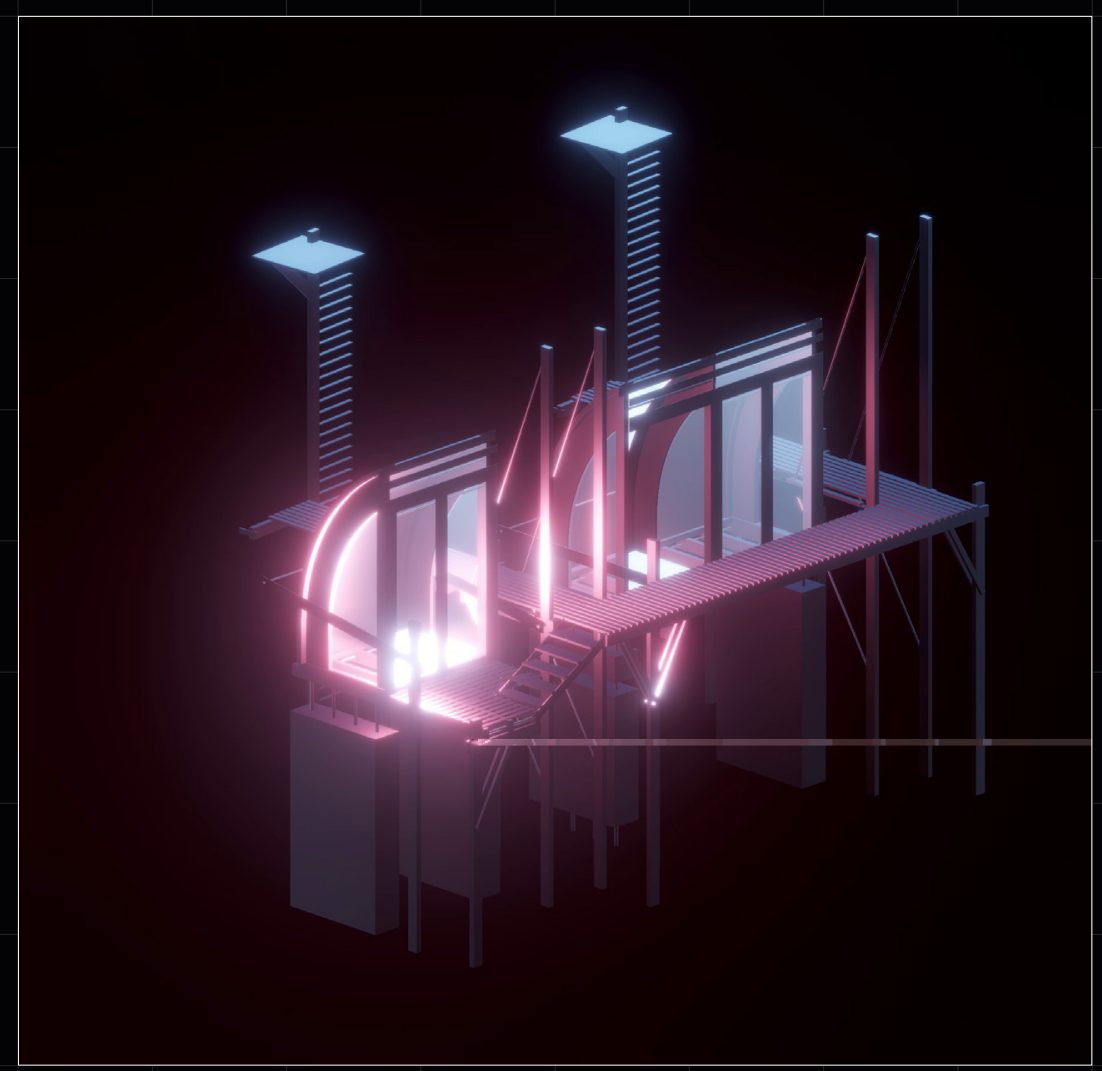

(D) Refer to video file 

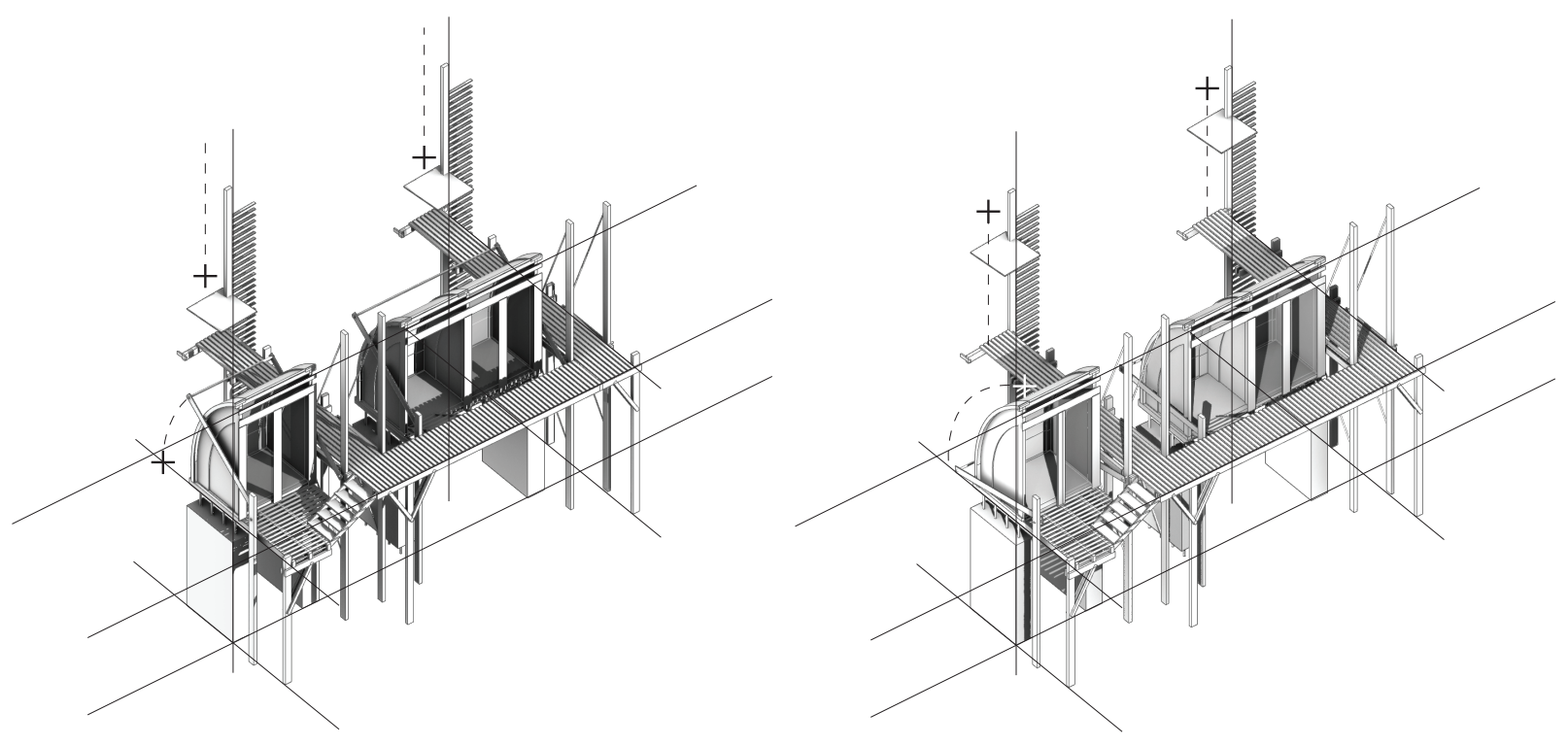

Fig. 66. Notation Diagram of Shift in Spatial Condition_\#7 The Enclosure

Image by Author

THE ENCLOSURE explores the notion of transitioning not as a physical entity but as properties of light to permeate through physical barriers and extend its range, lighting up surfaces from a distance.

The formal construct of this exploration piece extracts elements from the artefact model. The elevated binary forms reflect the two elbows with the propped stilts reflective of the abstraction of a table in the artefact model. The constant hinging of the arm frames convey architectonic translation of the lowering of elbows as a hinged entity. 


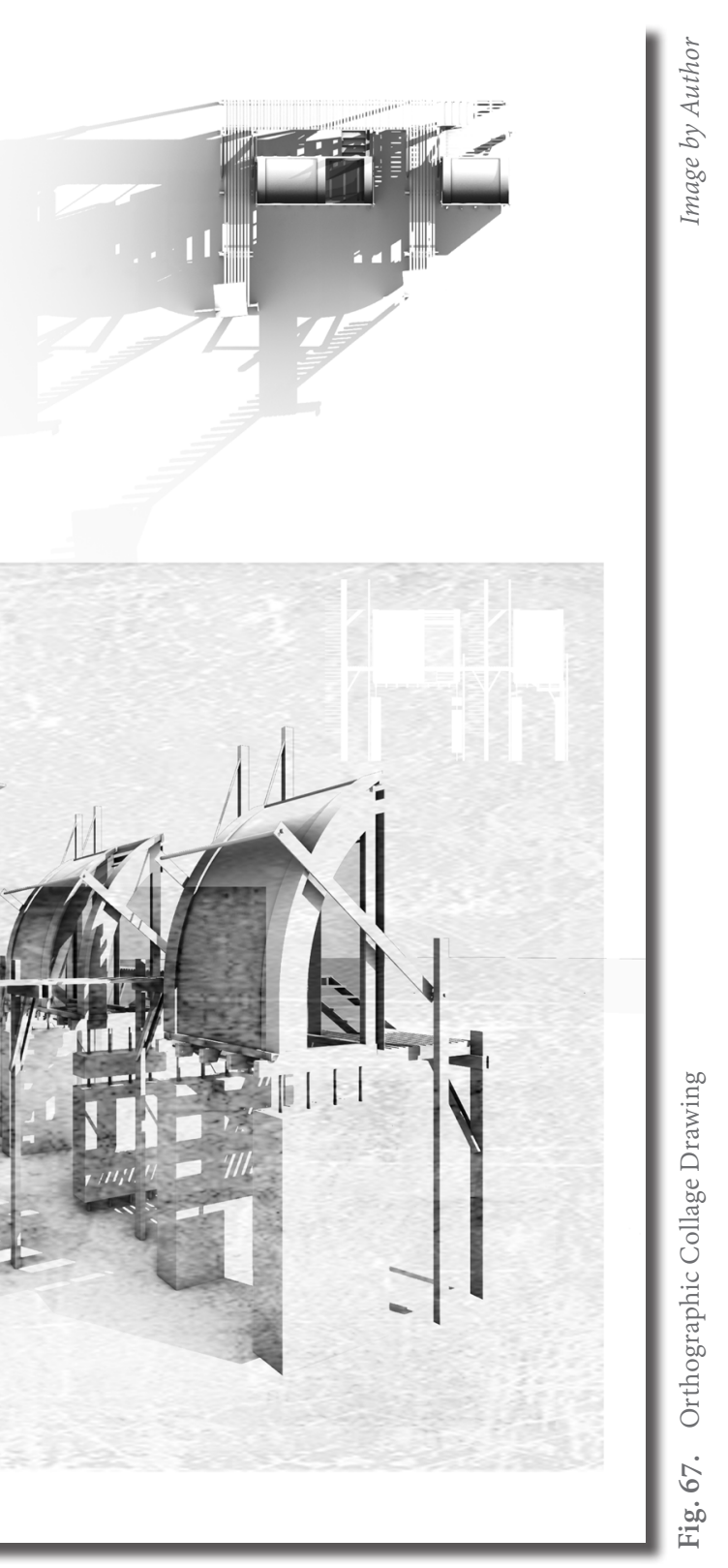




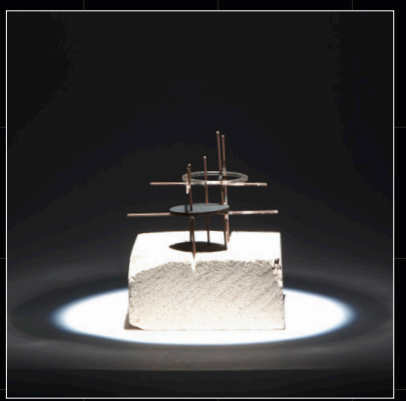

based on artefact 8. Twitching Eye

- A twitching eye means someone is missing them.

[Separation \& Longing]

PARTS OF A WHOLE HORIZONTAL SPACE VERTICAL SPACE
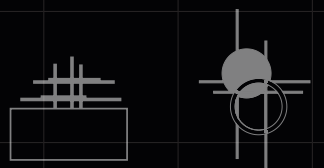

The notion that twitching eyes are believed to be caused by someone missing them from afar is reinterpreted as two beings that are isolated and kept apart, being incomplete without the other.
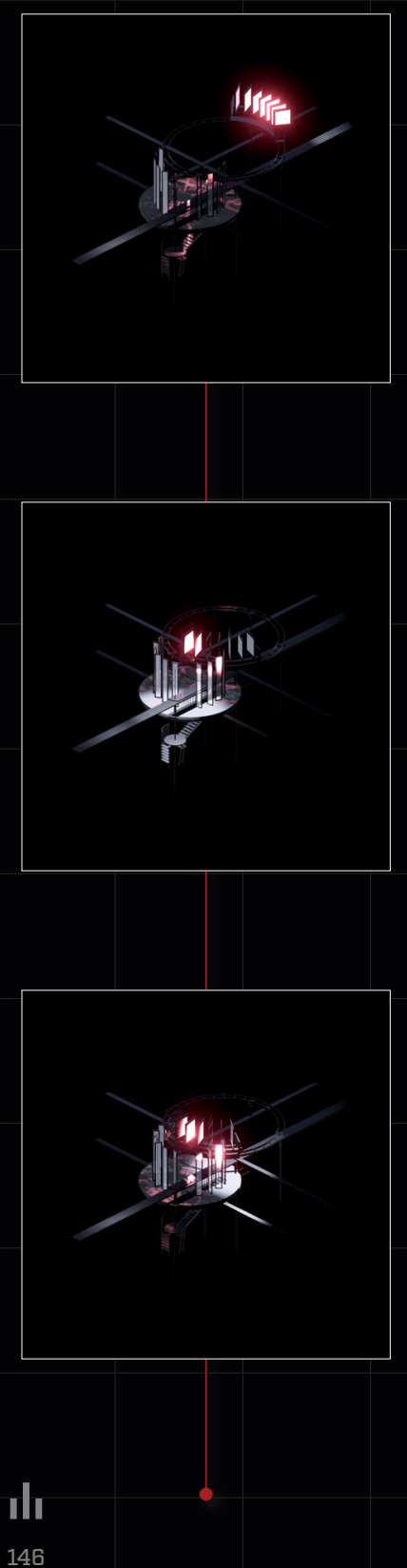

An array of glass frames on the lower tier rotate along their individual vertical axes while an array of glass frames on the upper tier slide along the railing track rotating along the central axis of the upper tier. The glass frames on the upper tier are reflective of the missing fragment of the lower tier that is required to form a complete circular arrangement.

This animated scheme employs this two-part system to symbolise the two beings that are shifted apart through vertical and horizontal spaceincomplete without the other.

A spotlight pans backs and forth across the scene depicting the notion of searching and longing.

A source of red light is projected onto the surfaces of the lower tier; however the top tier reflects this red light in some scenes to convey the notion that although the two are isolated, there is still a metaphorical element [light] that keeps them bounded together. 


\subsection{The Fromes of Isolation}

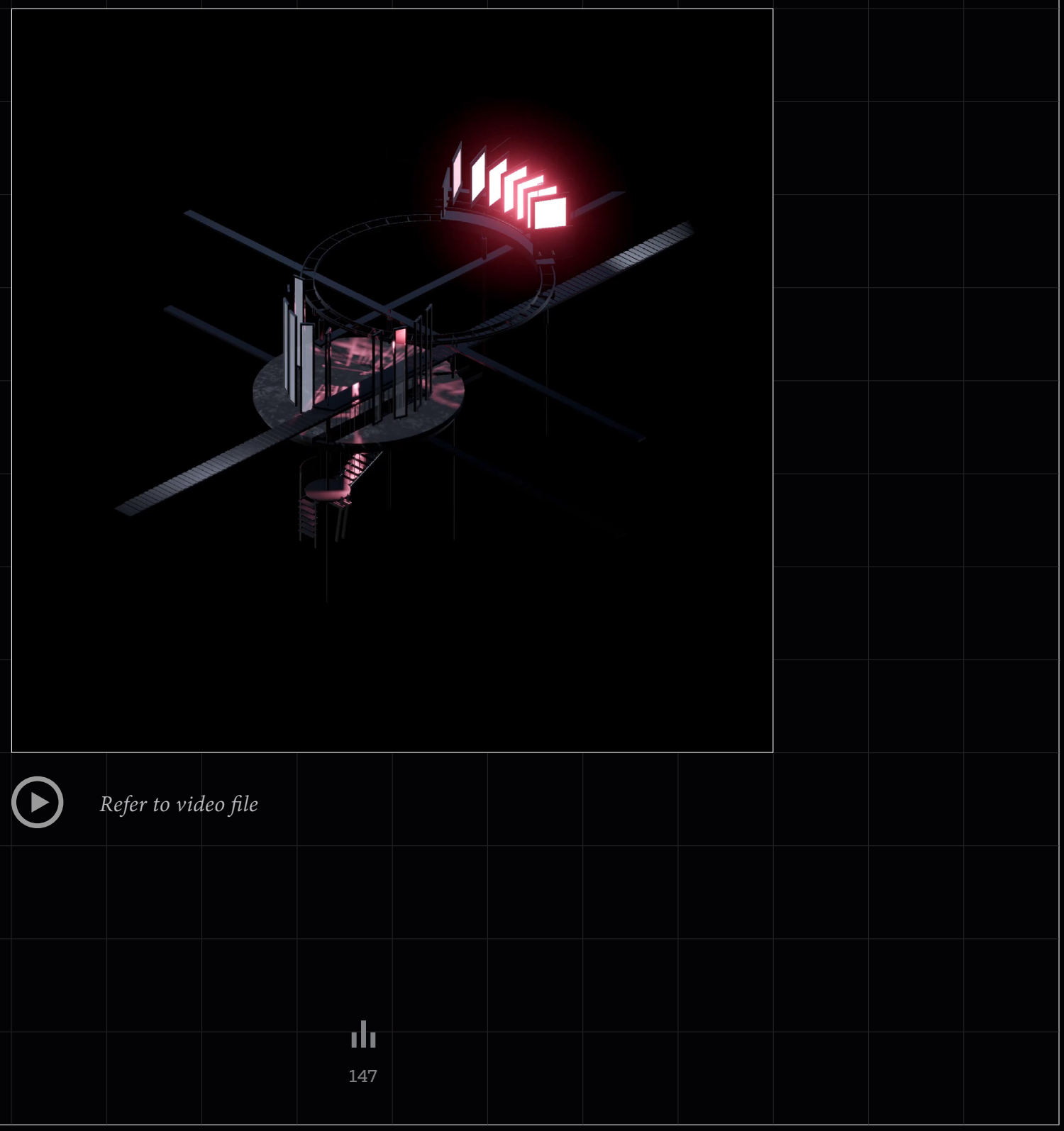



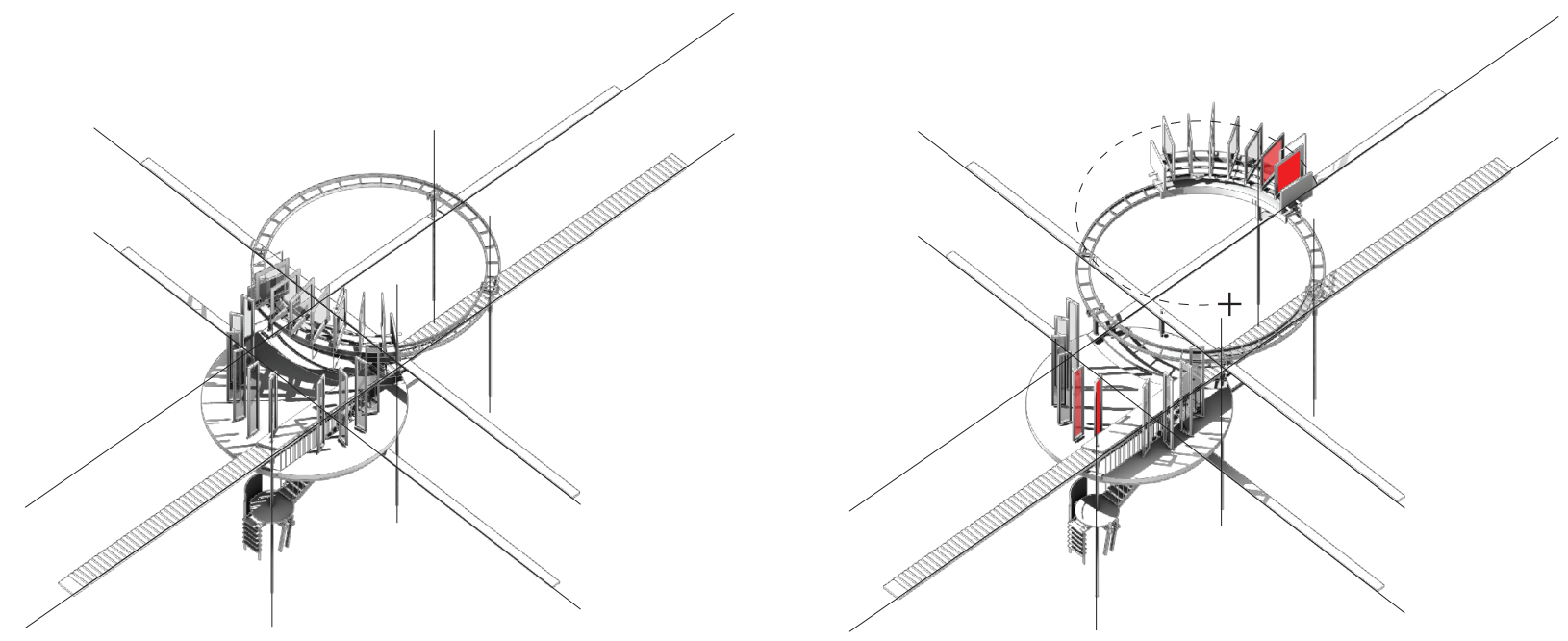

Fig. 68. Notation Diagram of Shift in Spatial Condition_\#8 The Frames of Isolation

THE FRAMES OF ISOLATION explores the notion of longing and distance through vertical and horizontal spacing, fragments of a whole and the referencing of duality.

The formal construct of this piece extracts directly from its artefact model, where the two circular entities are adopted to be the two tiers each housing frames that would reflect and reference each other as the two beings that are of one but never meet. 


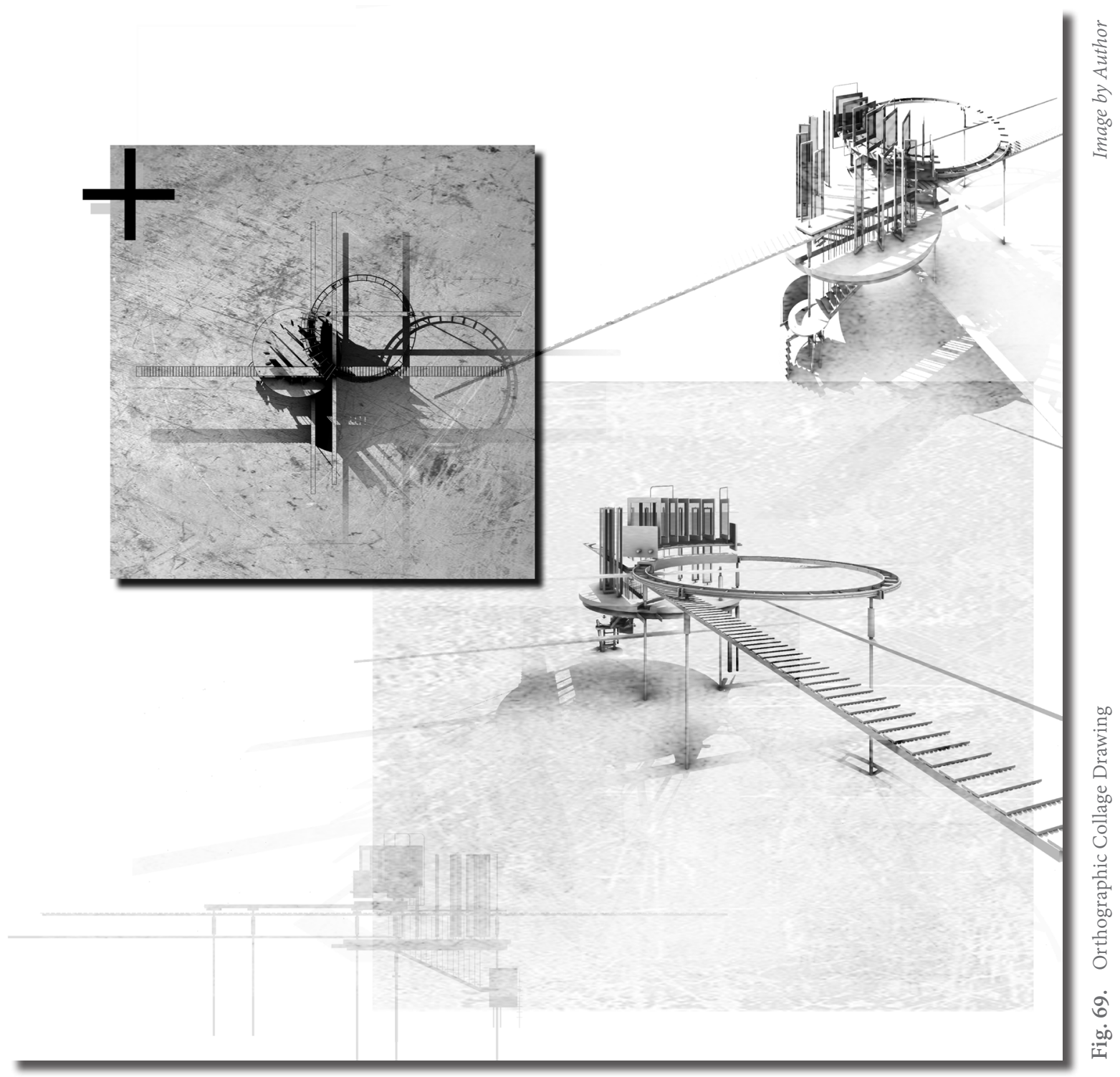




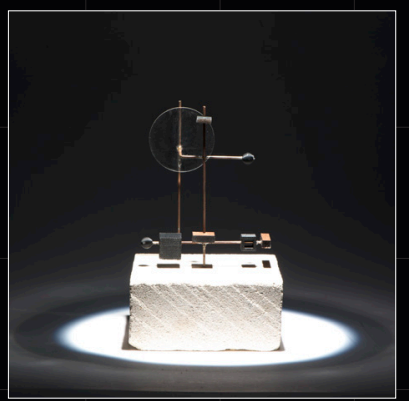

based on artefact 9 . Sleep Porralysis

- When one experiences sleep paralysis, there is a ghost sitting on them.

[Paralysis \& Lack of Control]

\section{$S T A T I C$}

$F$ I $X E E$

HORIZONTAL STILL

VERTICAL PRESS
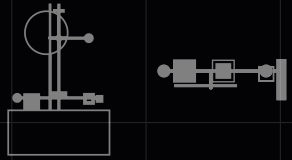

This animation scheme starts off with a boxed entity entering the scene from above and resting down on the horizontal subject below.

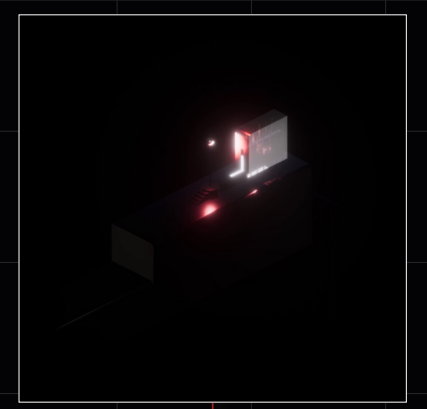

This action depicts the pressing down of a ghost figure during sleep paralysis as believed in the superstition this scheme is based on.

A rectangular light entity passes across the scene where it slots into an opening, setting off a trigger where the ground partially submerges, revealing an underground spatial layer.

The notion of the above and below is representative of the consciousness and subconsciousness. The light setting off the trigger reflects the state of sleep at the moment when one experiences sleep paralysis. A cluster of projected red lights is also present at this moment to enforce the notion of chaos and nightmare.

As the box figure returns upwards, no longer pressing down on the subject, the light entity makes its way out through a final gateway. The light begins to fade out and the entire scene dims away-conveying the notion that the paralysis and nightmare have now passed. 


\section{${ }_{3.29}$ The Dormant Crypt}

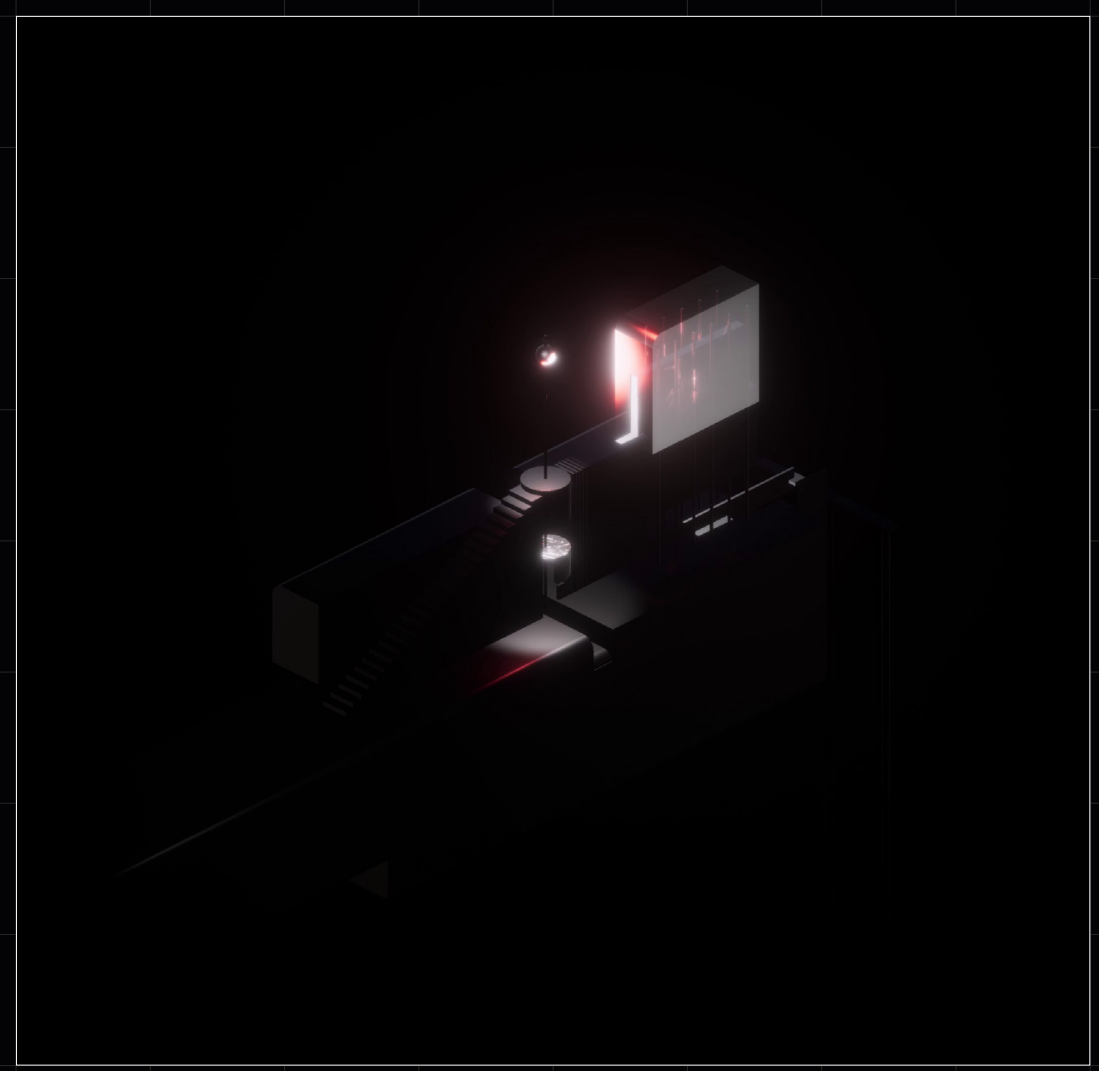

(D) Refer to video file 


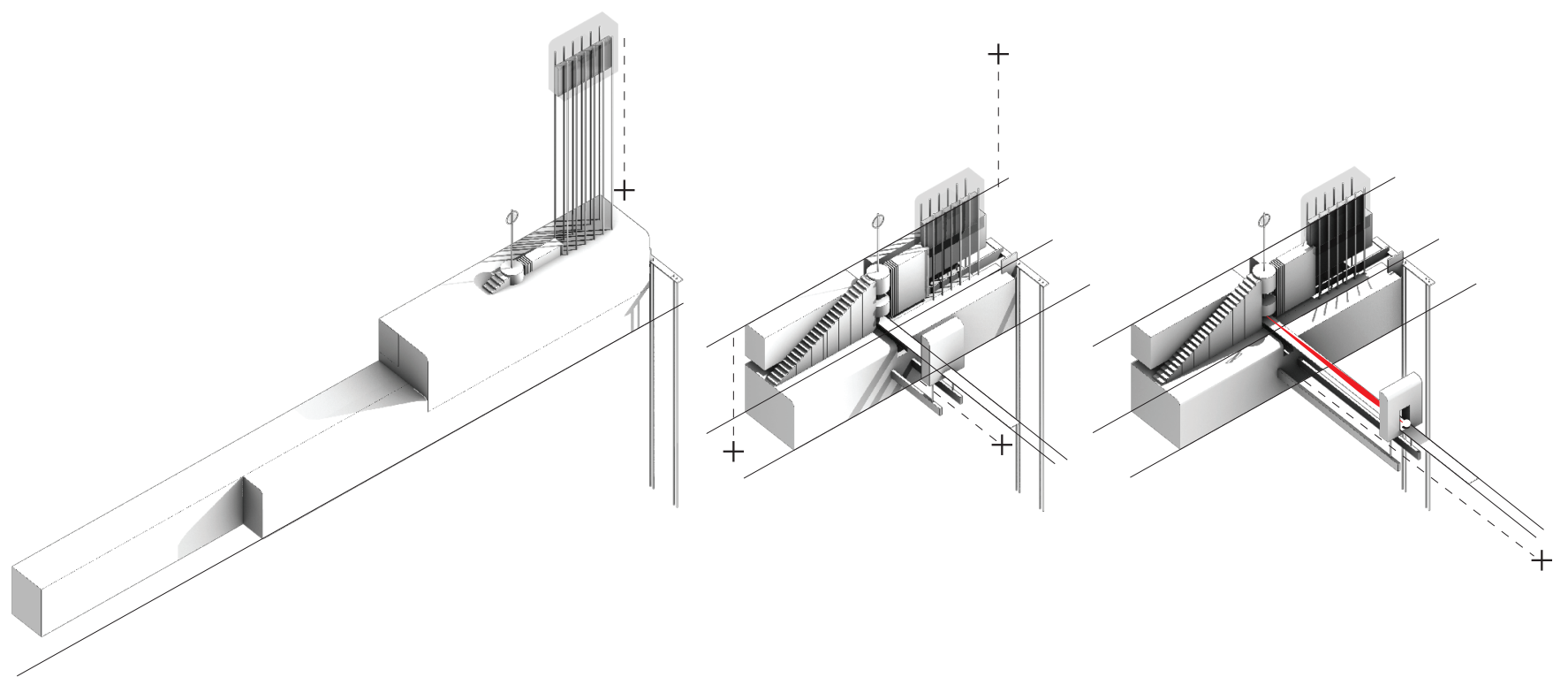

Fig. 70. Notation Diagram of Shift in Spatial Condition_\#9 The Dormant Crypt

Image by Author

THE DORMANT CRYPT explores the notion of sleep paralysis and the unconsciousness through the formal and spatial shift of its constituent parts. It employs notions of being still and fixed as the interpretation of sleep paralysis, triggered through the event of a pressing down figure. It also employs light as an indicator of the unconsciousness entering and exiting the stages of sleep. The shifting light and the revelation of transitional elements offer opportunities as navigational guides for the next stage. 
$+1$

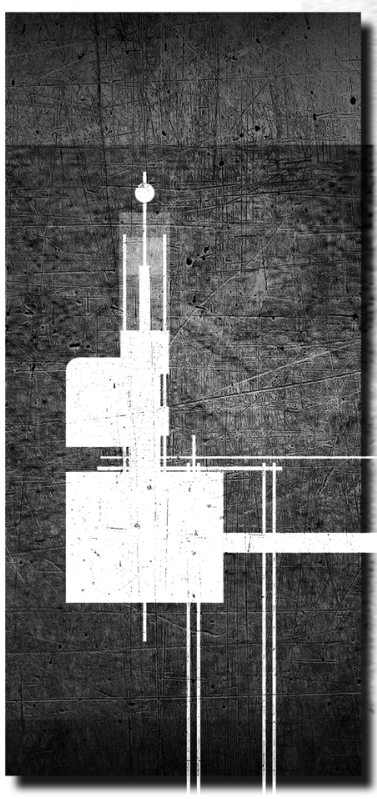

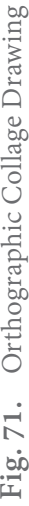


Further Design Exploration: Hologram

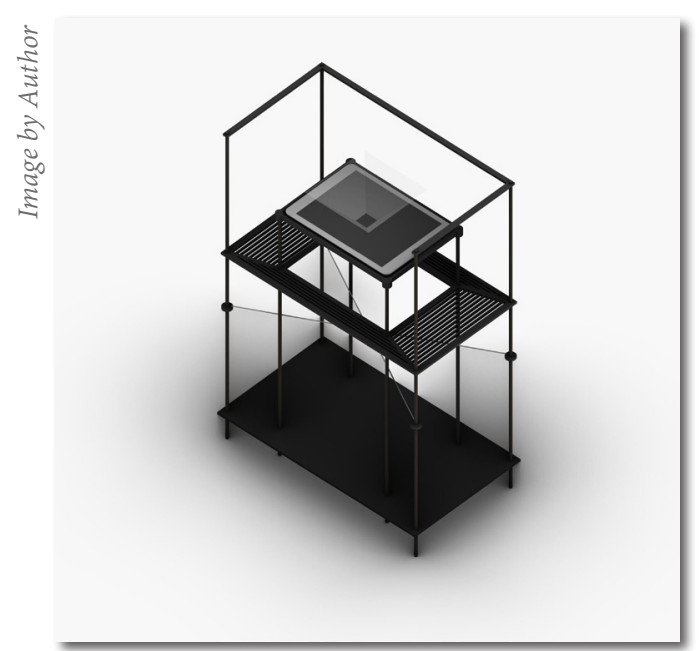

Fig. 72. 3-Dimensional Overview of the Holographic Plinth

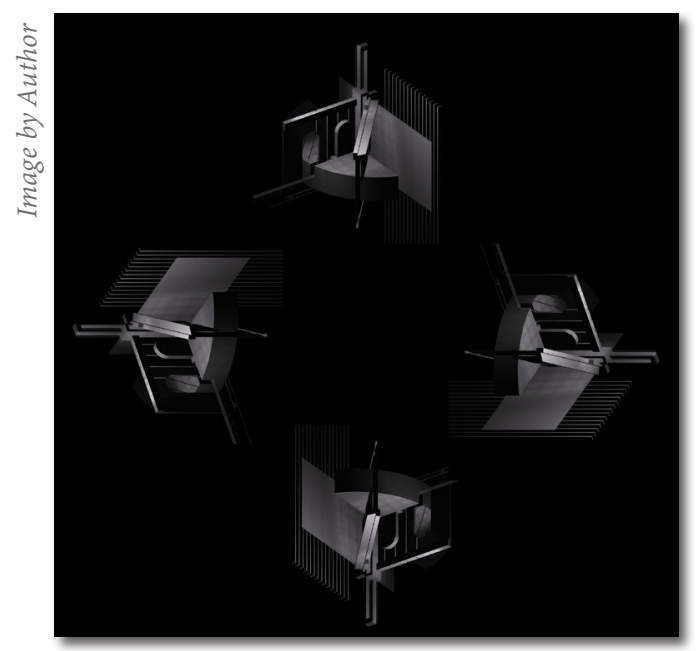

Fig. 73. Digital Holographic Screen
The hologram is a design exploration that I undertook in this series as an attempt to continue to transcribe the drawings from one medium to another. The analogue models developed into drawings and into digital animationsthe physical moved into the realm of the virtual. This exploration attempts to move the digital animations back into reality, taking on a pseudo-physical form. The animations become somewhat alive.

This is achieved through the assembly of the following elements:

- Holographic Pyramid constructed using perspex sheets

- Digital Screen: iPad

- iPad stand: mounts the screen flat allowing the pyramid to sit horizontally on top, encased within a black backdrop to control the lighting

The animations are collaged into 4 sections and reflected onto the 45 degree angle of the perspex pyramid, making the animation appear as one "floating" holographic projection inside the pyramid.

This exploration piece did not contribute much to the next stage of the design-it was however a useful tool for displaying spatial concepts bound in time by actuating the concepts as "floating" in space. 


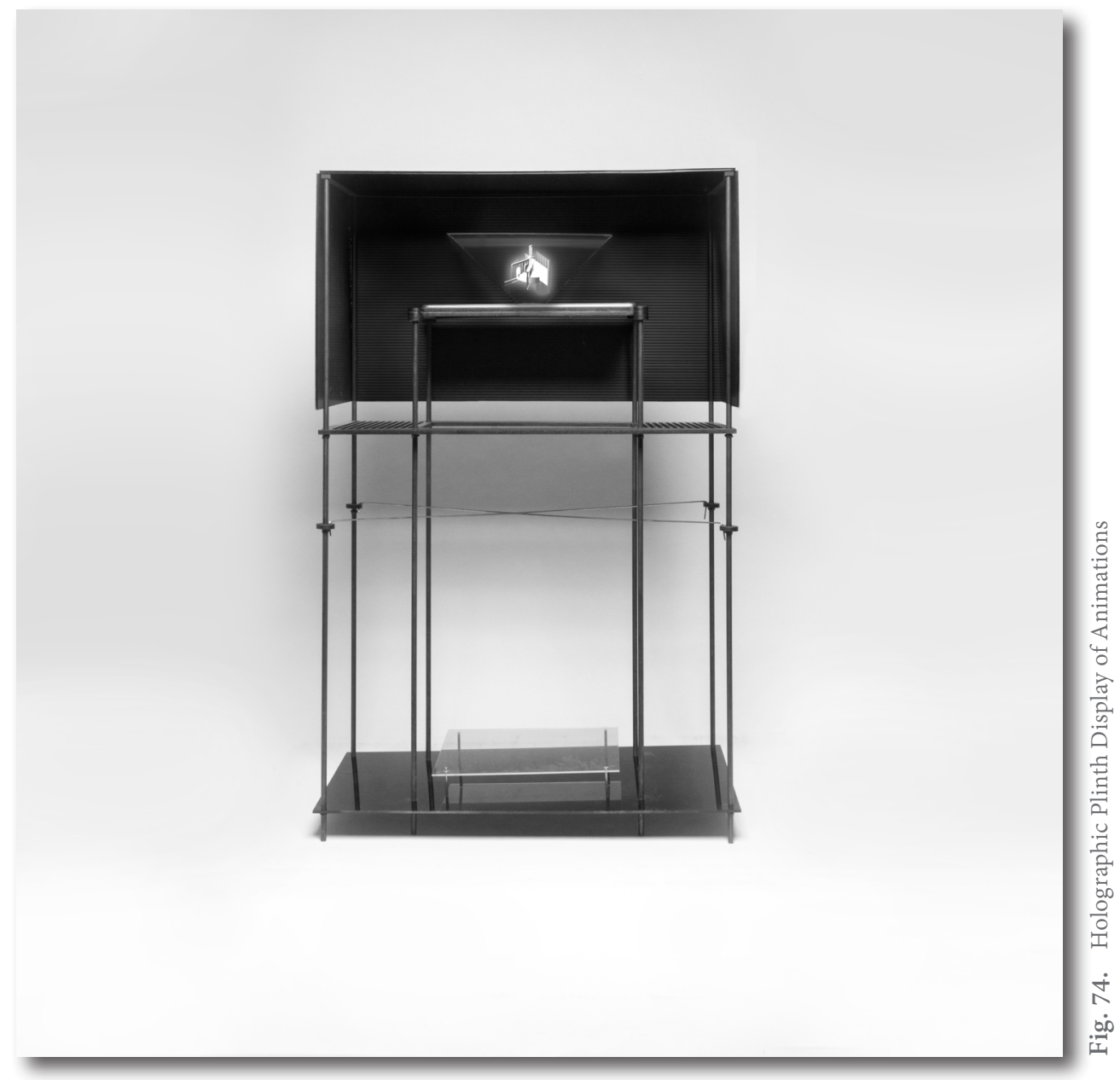

(D) Refer to video file 



\section{Critical Reflection}

\section{REFLECTION ON DESIGN REVIEW 2: FEEDBACK AND DISCUSSIONS}

A formal Design Review for Design Stage 2 was held on 9 August 2019. The conversation centred around the interrogation of an architectural programme and the pushing of boundaries.

\section{- Architectural Programme}

Programmatically unclear about how the interventions are intended to be inhabited-is it important that they do and do they ever intend to?

The project looks at ways of interrogating architecture through the digital medium where we are no longer bounded by real world constraints such as gravitational forces and the fundamental notion of architecture being a fixed entity. Because the fundamental characteristics of architecture shift in this digital environment, there is no need to define a real world programme to the interventions. It becomes of 'other'.

The project initially struggled to fit programmatic function into the conceived interventions but through determining their purpose as purely speculative explorations to interrogate spatial concepts through the shift in time, it was justifiable that they do not house a traditional programme.

\section{- Pushing the Boundaries: Digital Medium}

Utilise the possibilities available through the digital medium to push the boundaries of architecture to the extreme and challenge every notion possible so that this is not just a standard digital environment with regular behaving architecture. Instead, do everything that architecture cannot do in the real world bounded by physical constraints to challenge and distort our experiential understanding of space.

This comment gave the project opportunities to really push the next series into testing irregular behaviours, where there is no concept of gravity or physical constraints in the digital environment. There is no need to replicate real-world physics but rather, the investigation utilises the lack of it as more of a feature to test spatial conceptual notions rather than seeing it as a flaw towards achieving realistic outcomes. 


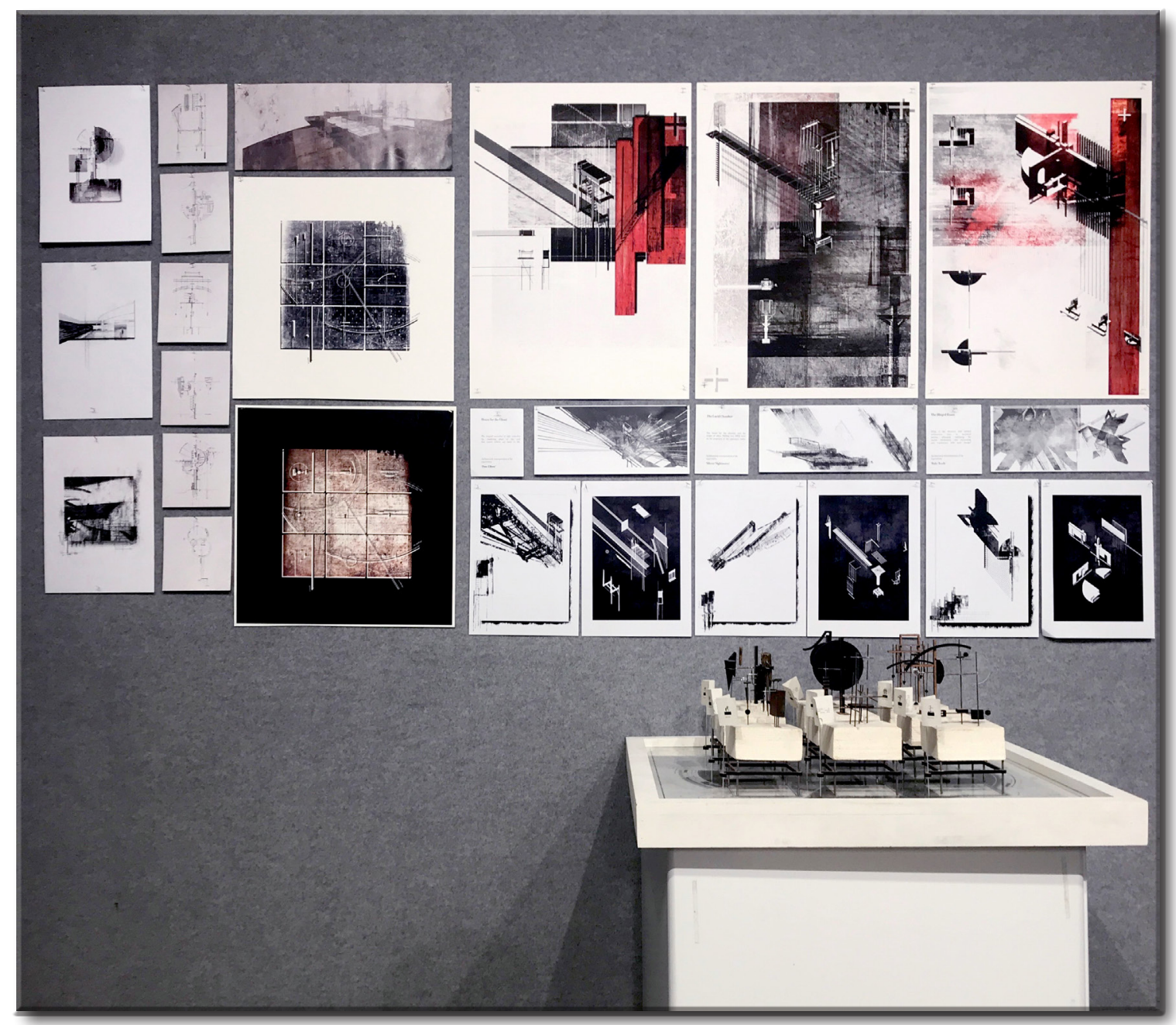

Fig. 75. Design Stage 2 Set of Drawings 


\section{REFLECTION ON PRELIMINARY DESIGN: DESIGN STAGE 2}

Design Stage 2 set out to explore how narrative architecture can activate allegory within the experiential as a tool for reinterpreting the notions of 'traditional' architectural elements through their shift in spatial conditions over time [RO 2].

Bruner's narrative diachronicity, as events unfolding over time, offered an interesting development of the artefacts-progressing them to express allegory [the metaphor of the superstitions they represent] through questioning and $r e$-interpreting architectural elements within a time-bound animated medium.

Some of the explorations were more successful than others in the sense that some offered up more opportunities for the next stage. These more successful ones exhibit implications of events that trigger the unfolding of other events. This gives opportunities for moving into the final design stage where the participant can be the player in the game that sets off these triggers, thus asserting agency into the shift in spatial conditions around them-each individual player constructing their own personalised experience.
The ones that evidence fewer implications of agency and the self being required to participate to trigger an event are still successful in the sense that they produced interesting formal and experiential outcomes through the shift in spatial conditions, as was set out to be achieved in the research objective of this design stage.

The design exploration series of Design Stage 2 set up 9 dynamic interventions to co-inhabit the game environment in the next stage, where the interventions can be experienced through a personalised narrative experience, by incorporating them within a digital gaming environment. 
曲 


\title{
4. Developed Design
}

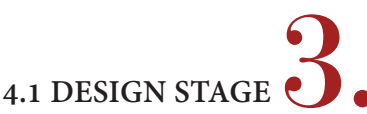

The Preliminary Design chapter summarised and critically reflected upon the iterative design experiments in Design Stage 1: Physical Models (Figurative Geometries) and Design Stage 2: Animated Space (Progress and Journey). The Design Stage 1 experiments were analogue and focused on RO1, exploring how culturally scripted ideas can be explored and expressed through allegorical methods. The Design Stage 2 experiments were digital and focused on $\mathrm{RO} 2$, looking at how narrative architecture can activate allegory within the experiential.

Design Stage 3 summarises and critically reflects upon the Developed Design: Game Environment (Invitation for Interpretation). It focuses on $\mathrm{RO}$, exploring how Digital Gaming Environments can be actively engaged to enable agency to actively participate in the experiential narrative outcomes. 


\section{Game Environment}

\section{INVITATION FOR INTERPRETATION}

RO 3: To explore how Digital Gaming Environments can be actively engaged in the architectural design methods and processes to enable agency to actively participate in the experiential narrative outcomes.

...The work succeeds if it triggers many interpretations, because it is not the artist alone who performs the creative act: the viewers make their own contribution. It is clear, however ... that he [the artist] sees the work presented as a riddle or an enigma not only to the viewer but also to the author. Therefore, the work of art

is an allegory in receiving, interpreting, but also in making.

- Haralambidou 230-231 
Design Stage 3 introduces the digital gaming environment as a medium within which the Design Stage 2 outcomes can be framed, experienced and tested in relation to narrative theory. It introduces self-positioning of the participant as a vital tool for interpreting and navigating through one's own experience within the constructs of architectural spaces. It aims to introduce a personalised experience that further challenges the conventional ways that architecture is perceived, with the intention that through this shift in perception, the elements of architecture in question could be further redefined throughthediverseperceptions ofvariousparticipants.

As Ganoe argues, our understanding of space is not exclusive; it is fluid and undetermined. Our interpretation of space is in a constant state of flux, as no two individuals share the same experience and perception of space.
The construct of the architectural experience in Design Stage 3 is founded upon Bruner's literary narrative theory and implemented through Ganoe's propositions interpreted within a spatial context.

The 9 interventions conceived through Design Stage 2 are organised within a fabricated context of a 9-square grid, where they each contribute their individual allegorical narratives conceived through the animated schemes in Design Stage 2 to the construct of the narrative experience of the game environment in this stage.

Their coexistence allows for opportunities for relationships and dialogues to be formed as a method of overlaying their individual narratives into one spatial experience. 

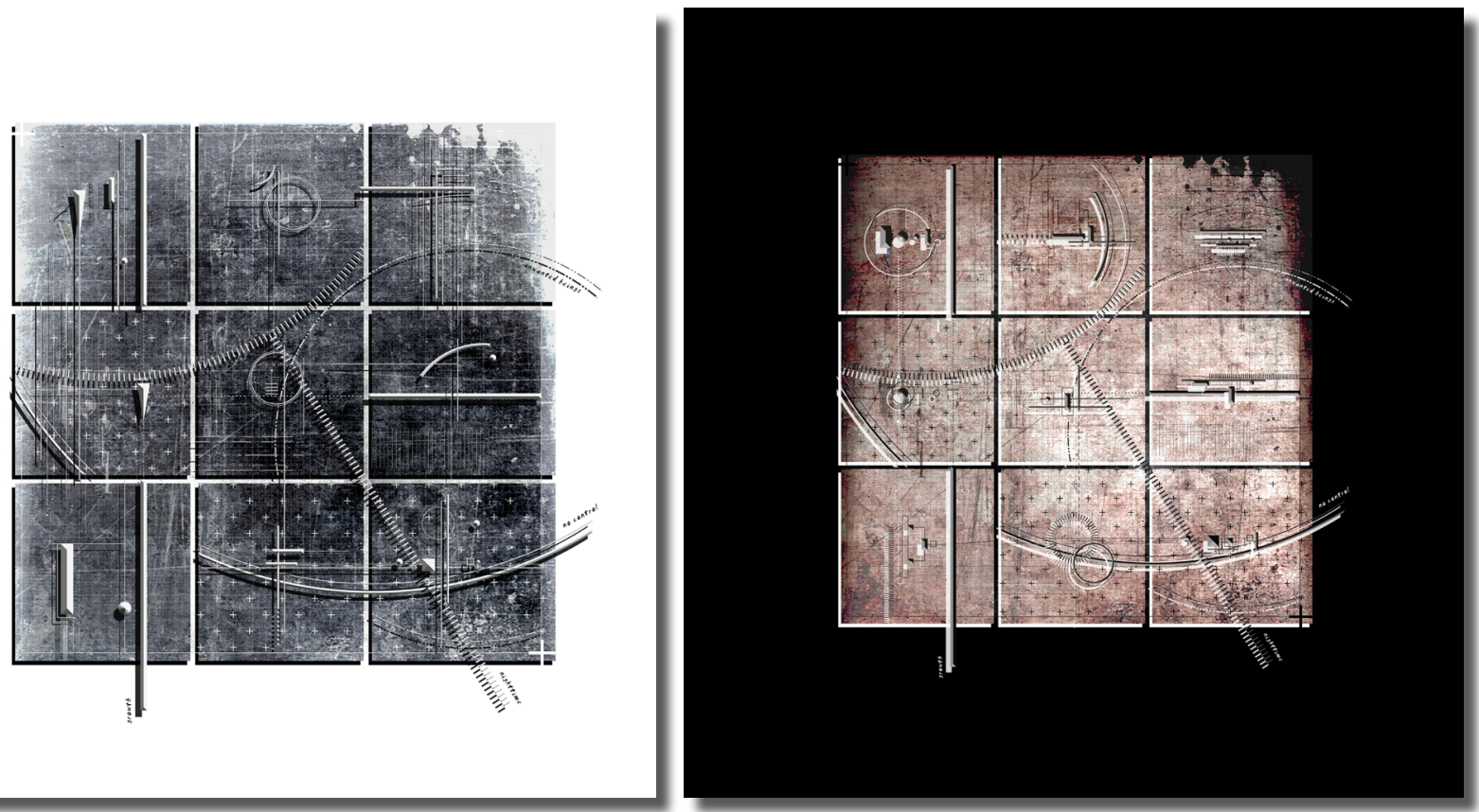

Fig. 49. Conceptual Notation Diagram Drawing Links between 9 Artefacts [\#1. Abstraction through Elevational view]

Fig. 50. Conceptual Notation Diagram Drawing Links between 9 Artefacts [\#2. Abstraction through Plan view]

Image by Author

Image by Author 


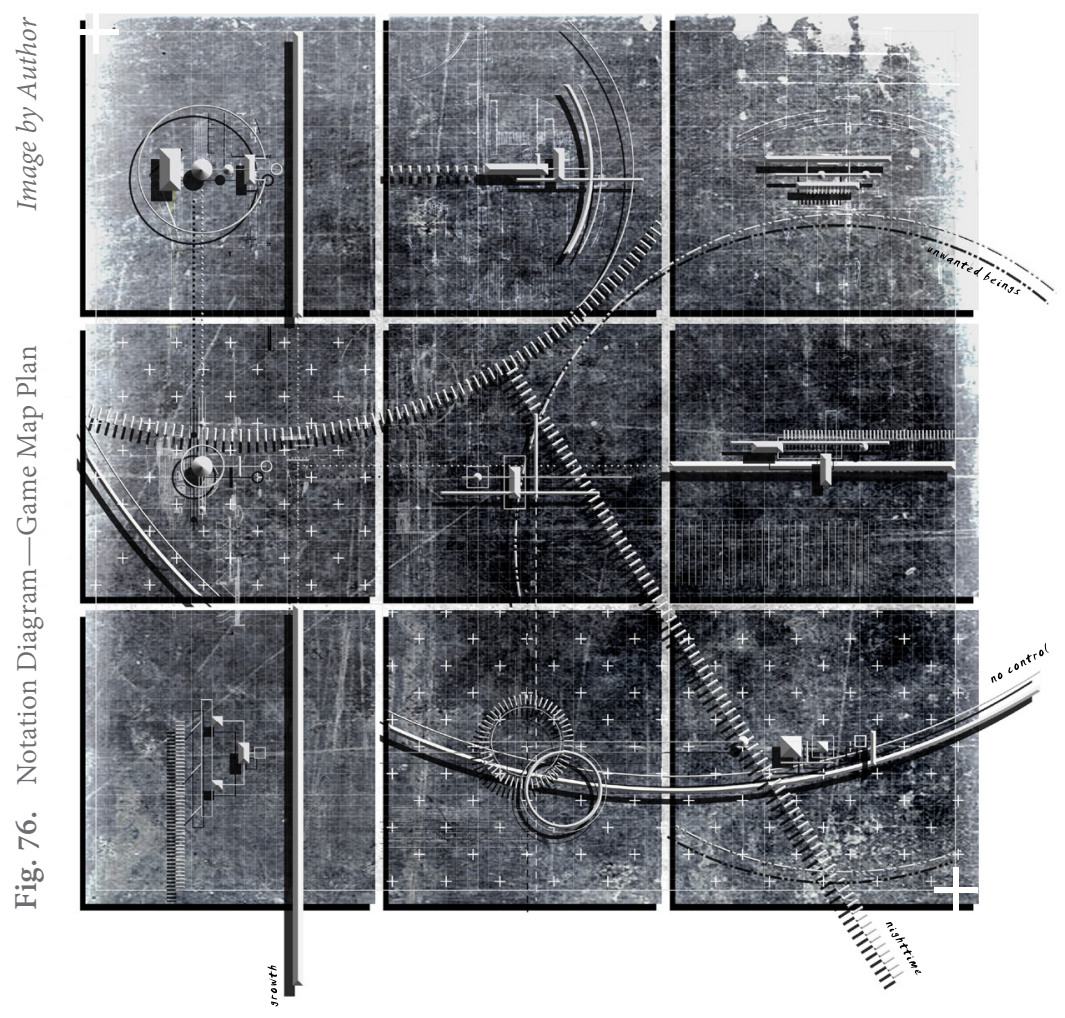

The diagram above organises the 9 interventions through 4 similar traits shared by their corresponding superstitions: night-time, growth, lack of control and entry. The diagram outlines how each intervention expresses these traits as well as aligns the interventions that share these similar traits.

Their common traits are mapped out through the lines and arcs in the conceptual notation diagram drawings (Fig. 49 and Fig. 50). These 2 drawings from Preliminary Design Stage 1 are combined into one drawing (Fig. 76), and this drawing is employed in this stage as the contextual site upon which the 9 interventions are situated.

The grid organises the system of the 9 interventions in their numerical order and their mapped out commonalities present opportunities for the conception of transitional elements as the formal manifestation of the links between the interventions. 

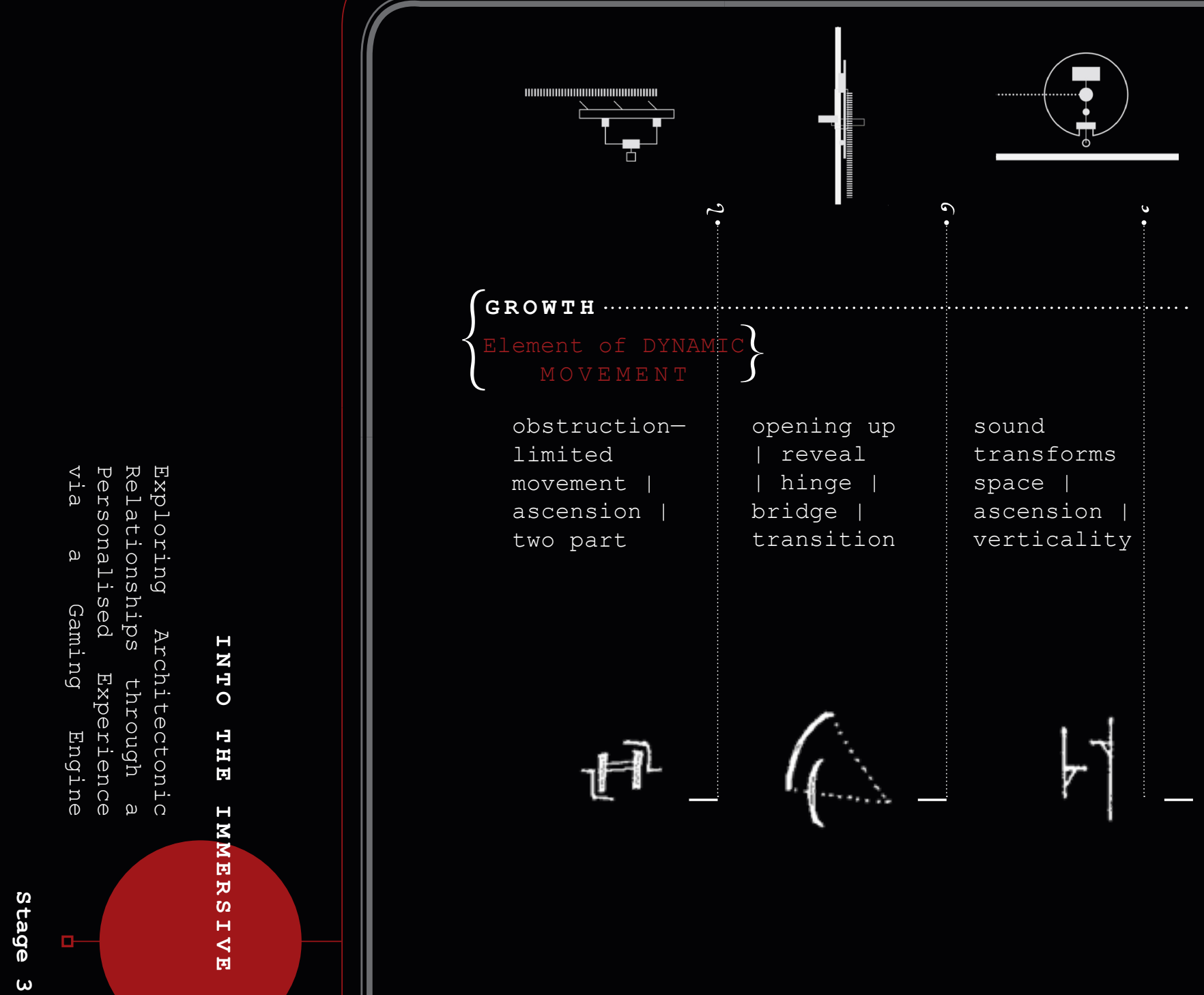


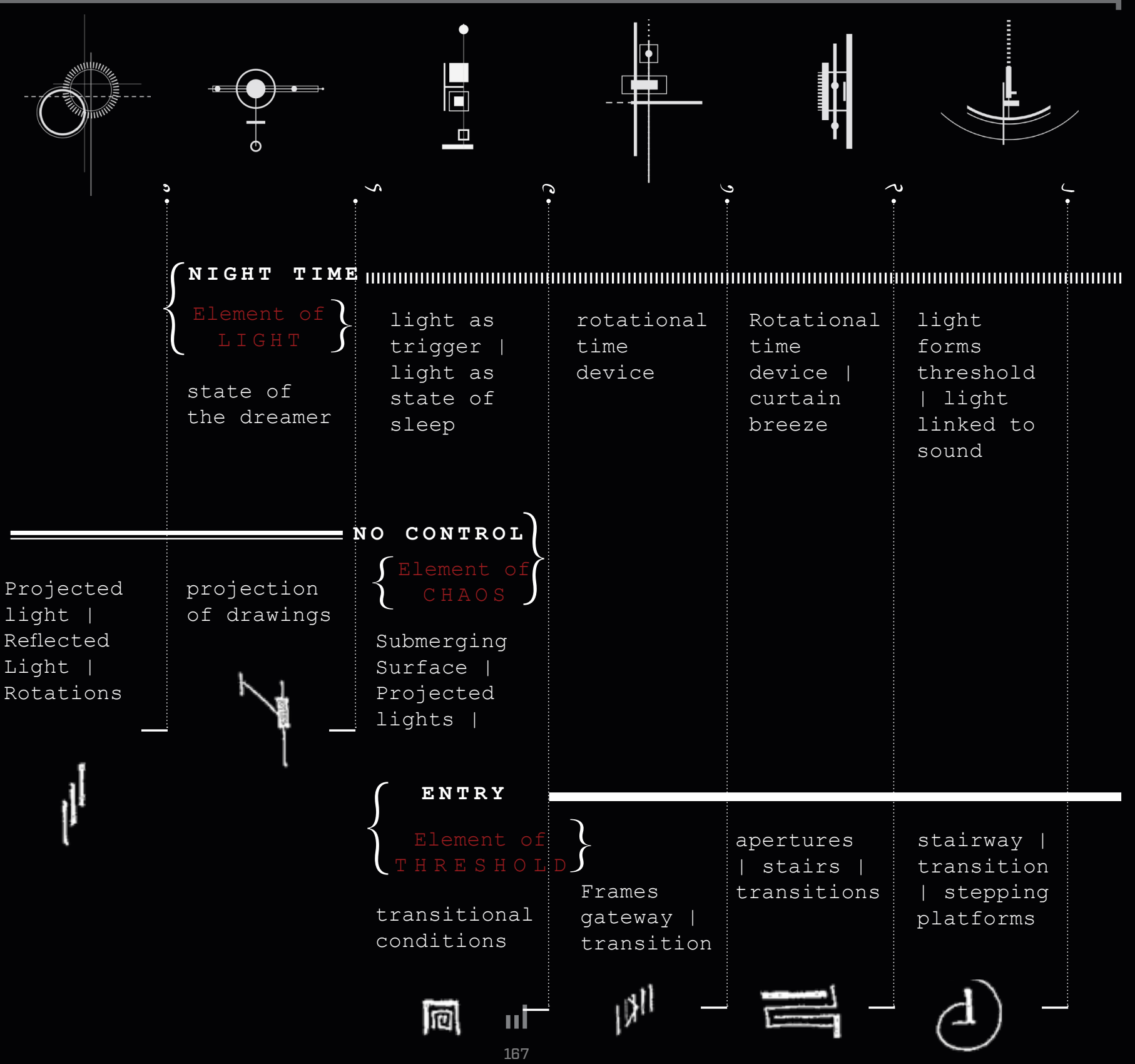




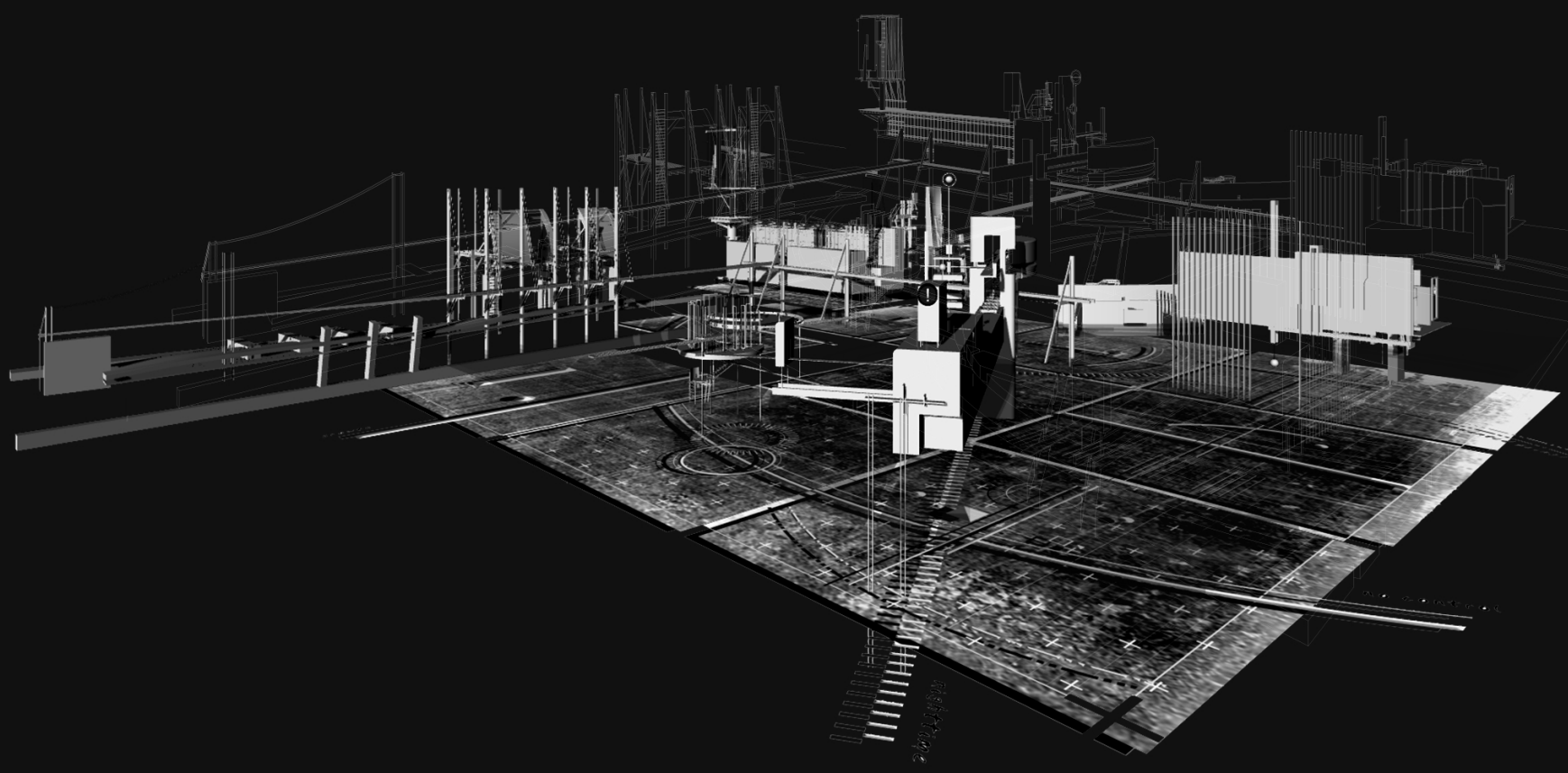

Fig. 77. Assemblage of the 9 Interventions within a Game Environment 


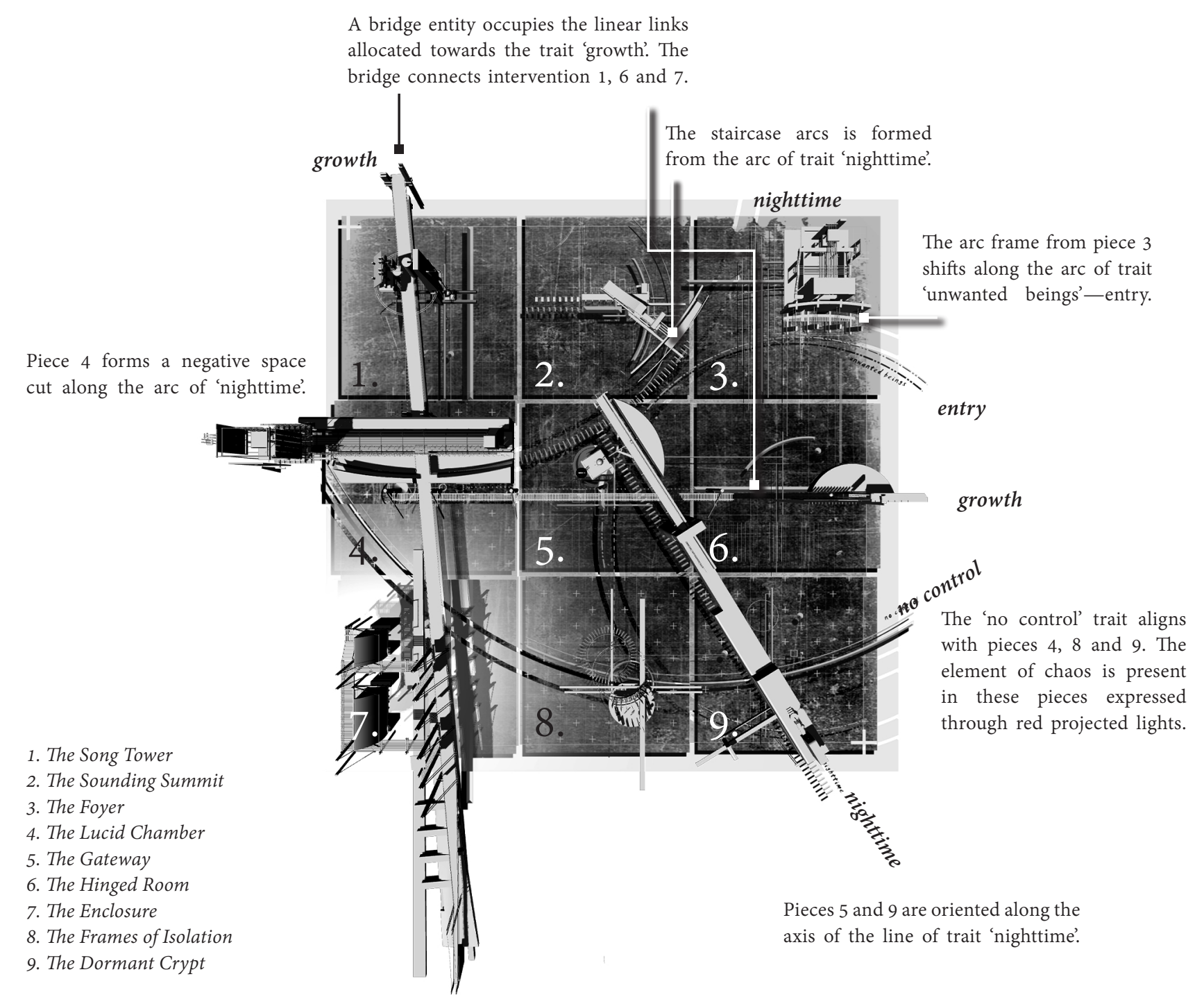

Fig. 78. Game Map Plan

This mapping device forms the gaming equivalent of an architectural 'masterplan'. 


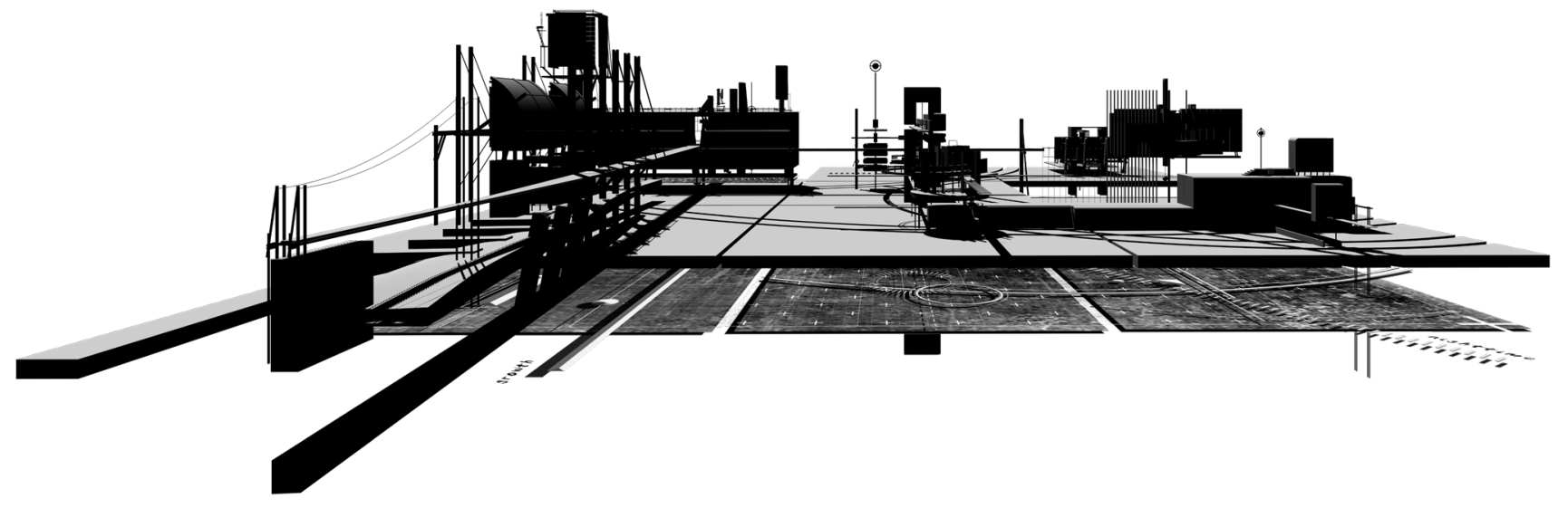

Fig. 79. Game Environment Layout Overview Image by Author 


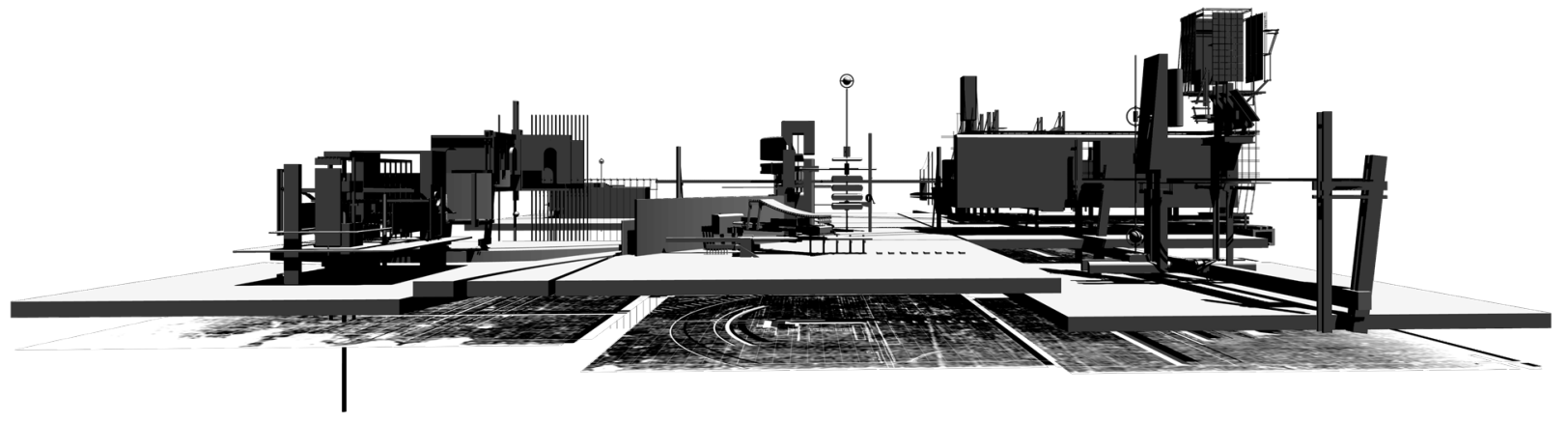

Fig. 80. Game Environment Layout Overview

Image by Author 
This conceptual Game Masterplan exploration was carried out in the early build of the game to test the spatial shifts and the organising of the 9 interventions to co-exist within the field of the digital game environment.

This animated exploration tests out how the relationships of the 9 interventions would transform to construct a narrative architectural experience.

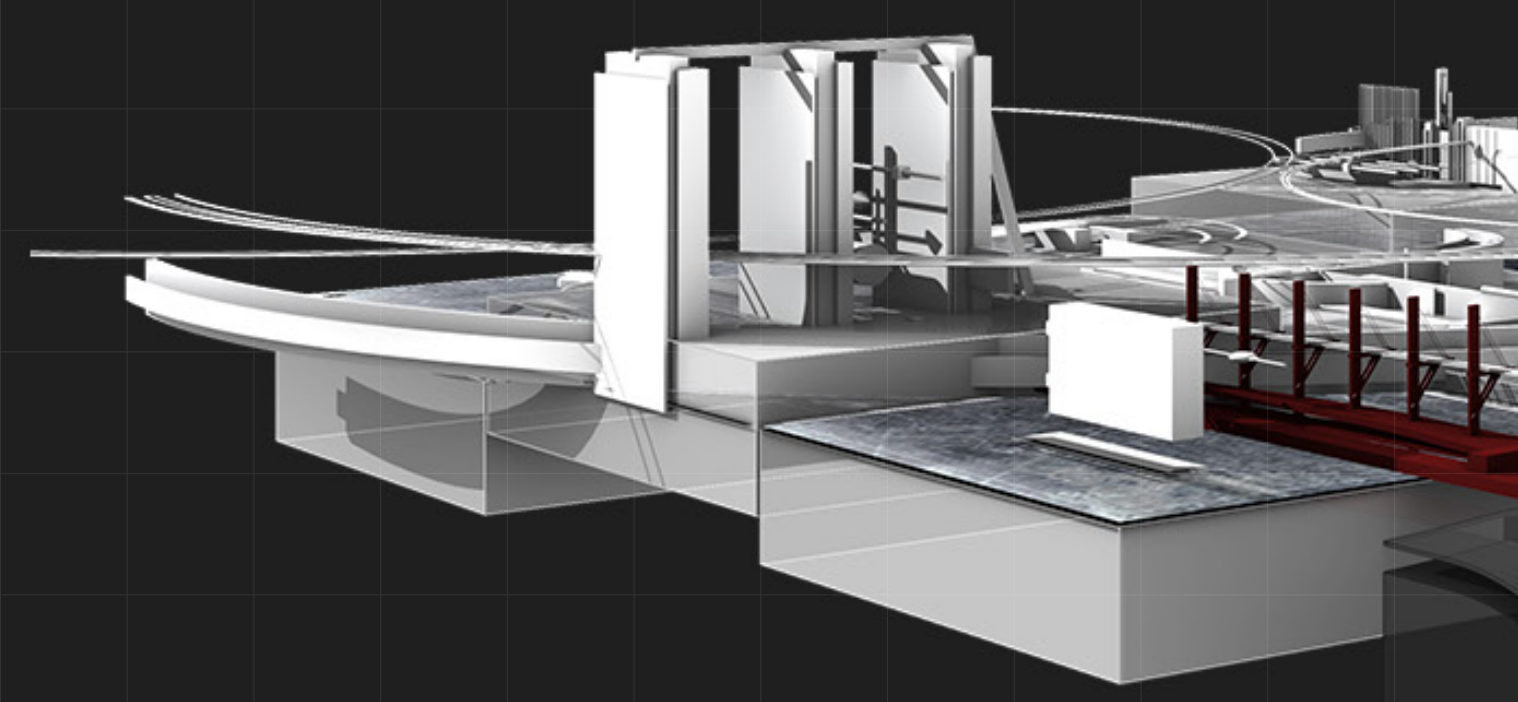

(D) Refer to video file

Fig. 81. Conceptual Game Masterplan with Player Route Mapping 


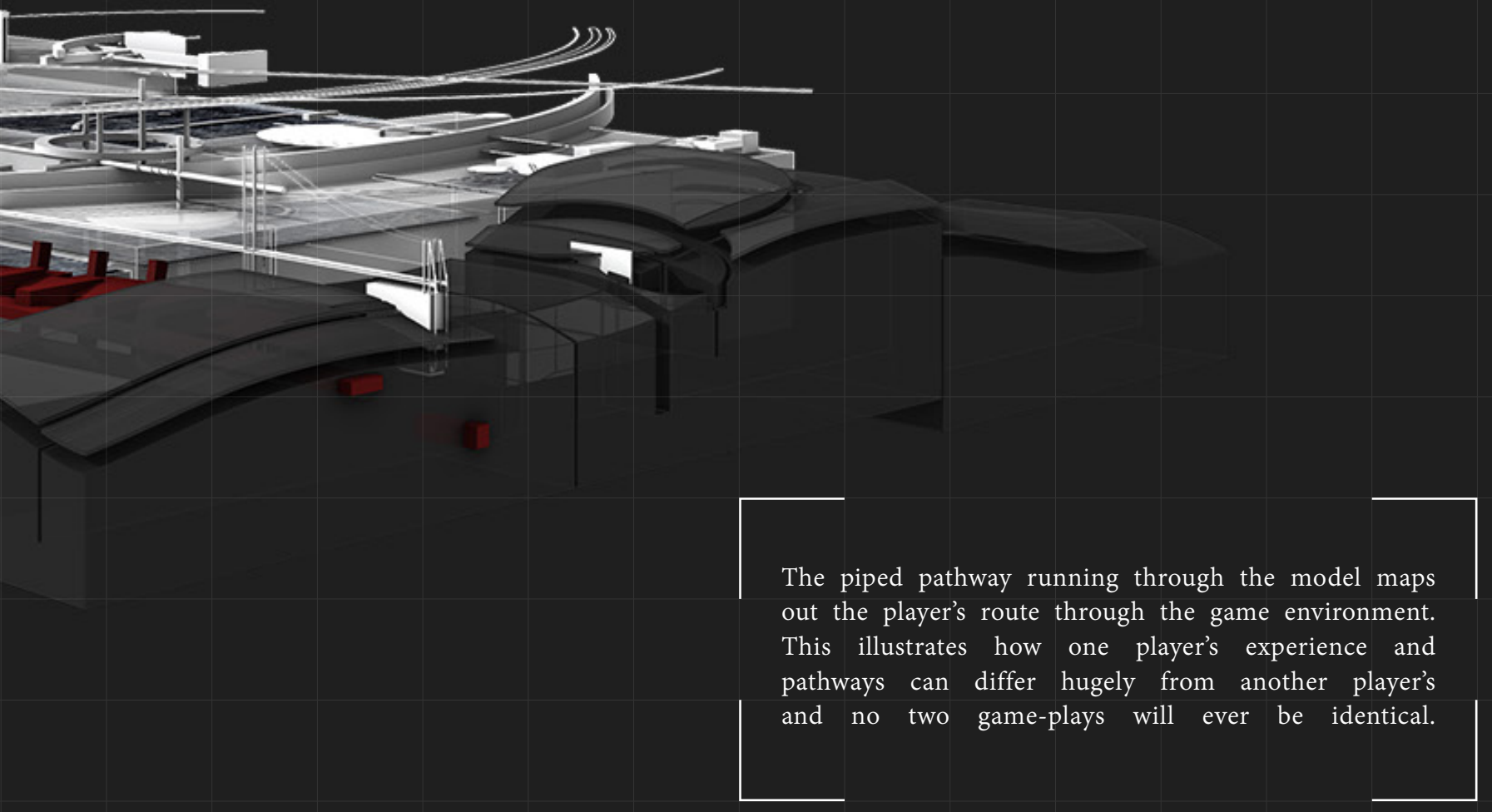


GAME-PLAY

117 
Through the element of agency, players are granted the tool and ability to navigate the game environment, constructing their own personalised experience. This shifts the explorations from the cinematic spatial eye [the experience of the viewer being directed by the camera as established by Eisenstein] and more towards the architectural spatial eye [the experience of space directed through own perception].

Two simulated game-plays are recorded in this stage to illustrate the two different journeys taken by two different players resulting in two completely different experiences of the spatial environment.

The player's route taken throughout the journey is mapped [blue light trail] and screenshots of the frames within the game-play are exhibited. 


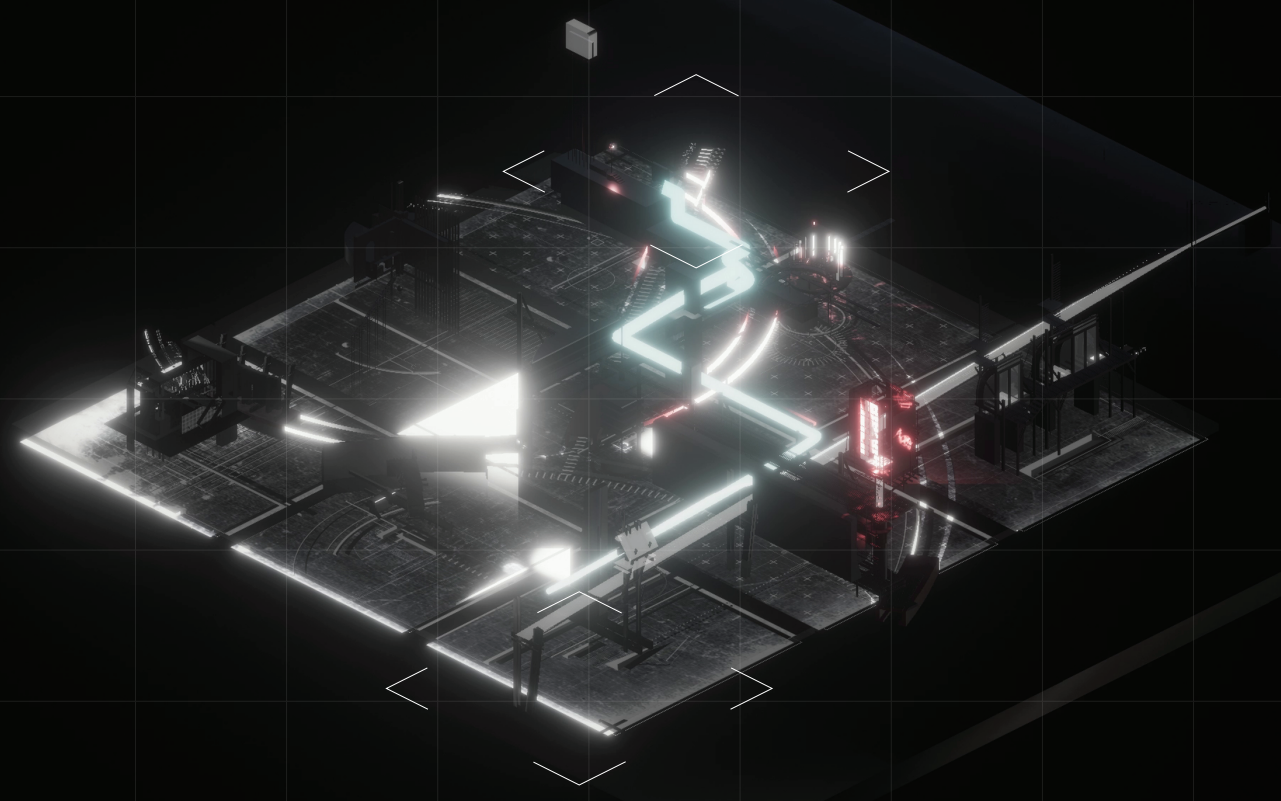

(D) Refer to video file

Fig. 82. Game-play Mapped Route 01 


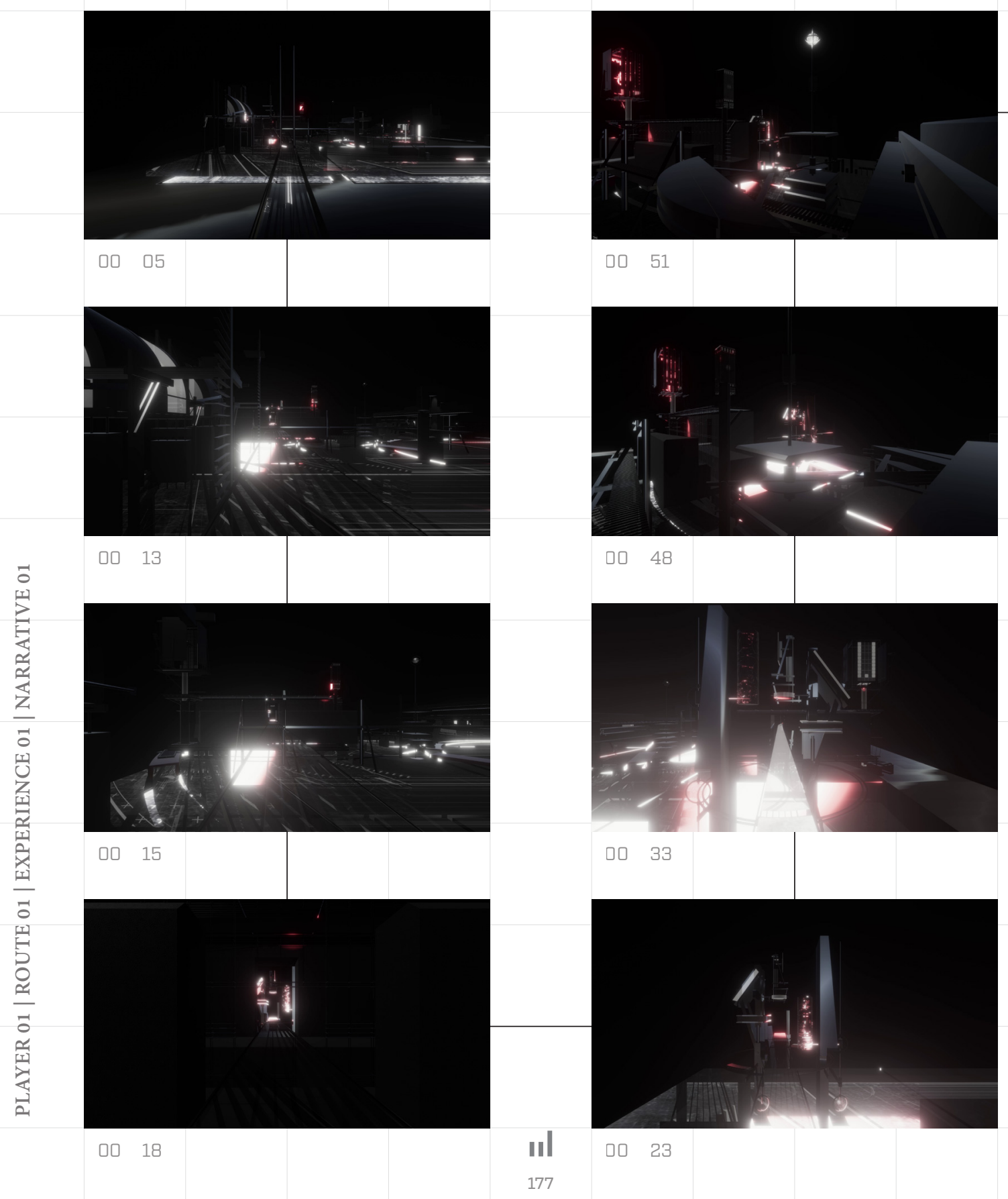



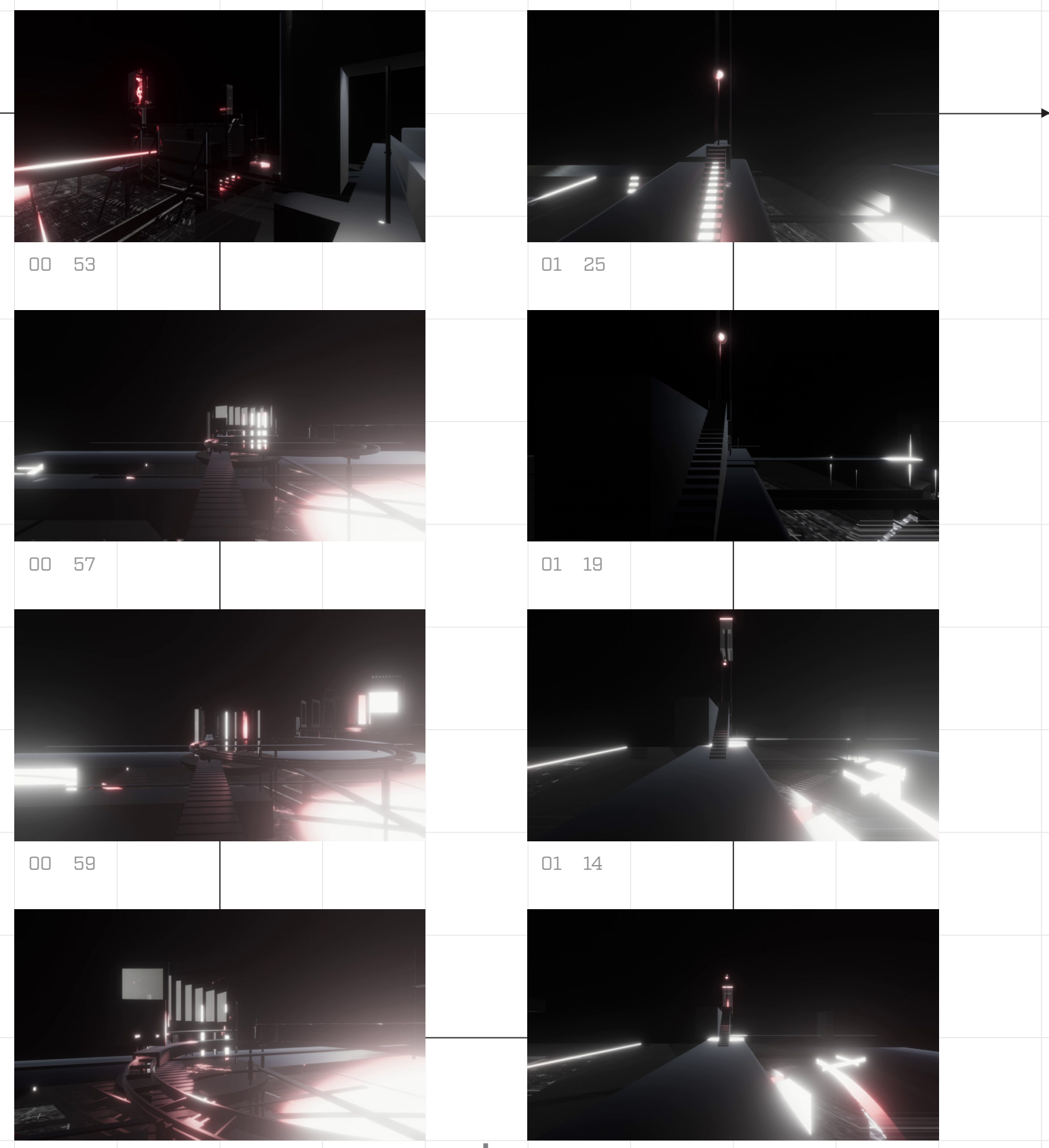

$01 \quad 02$
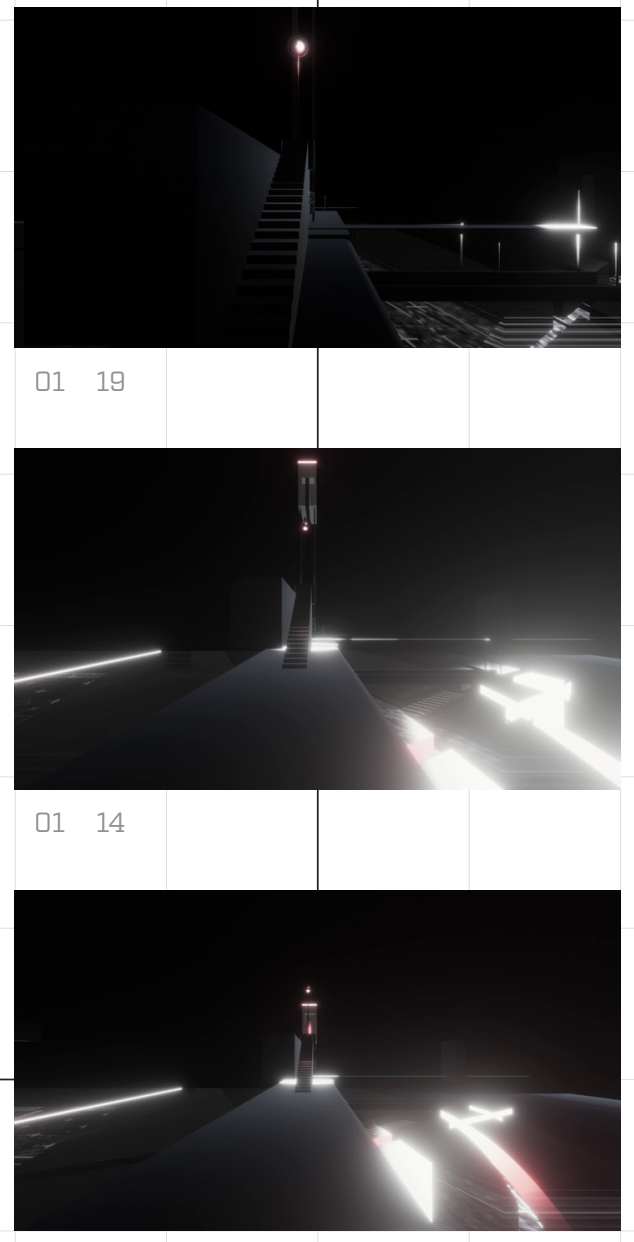

$01 \quad 12$ 


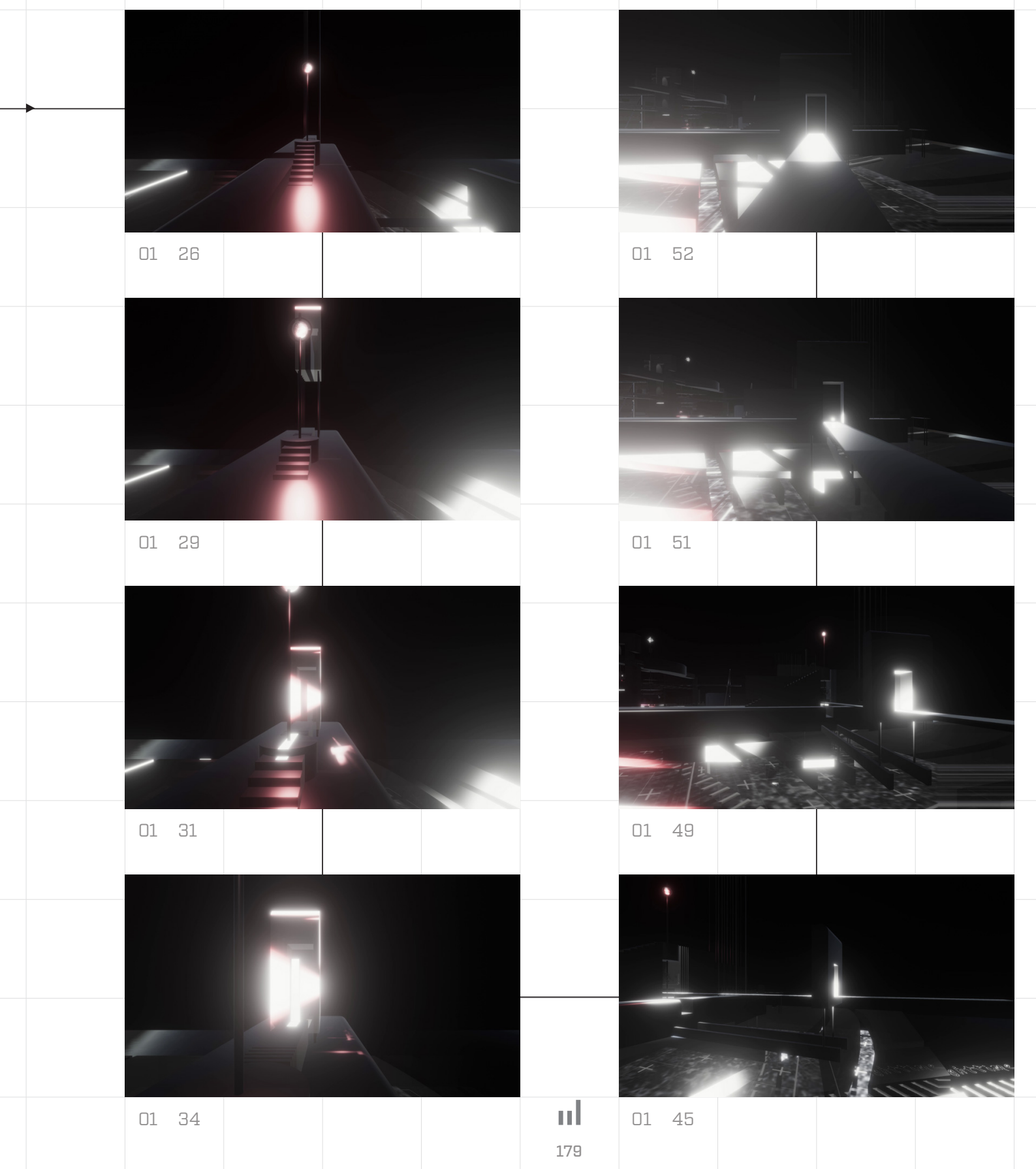




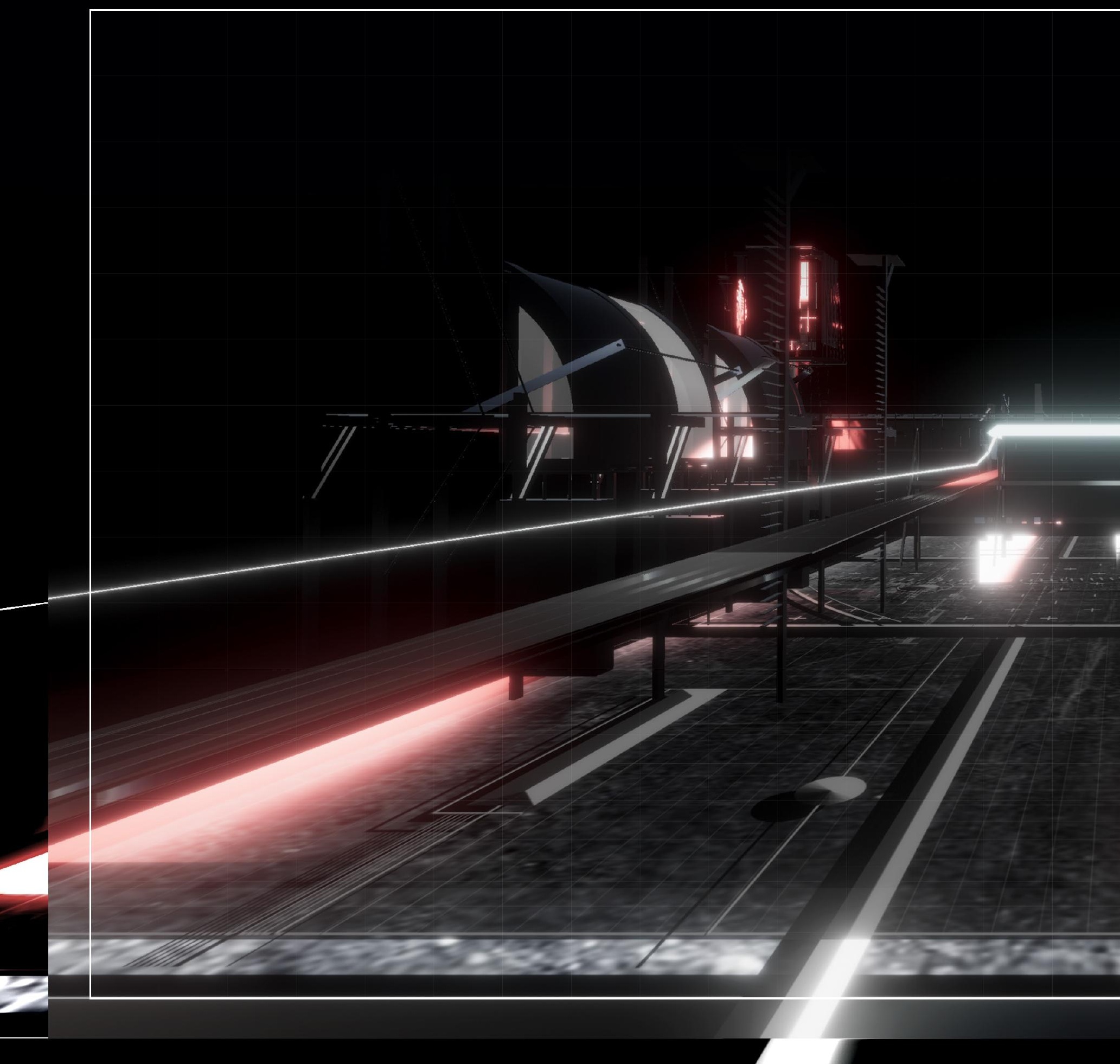



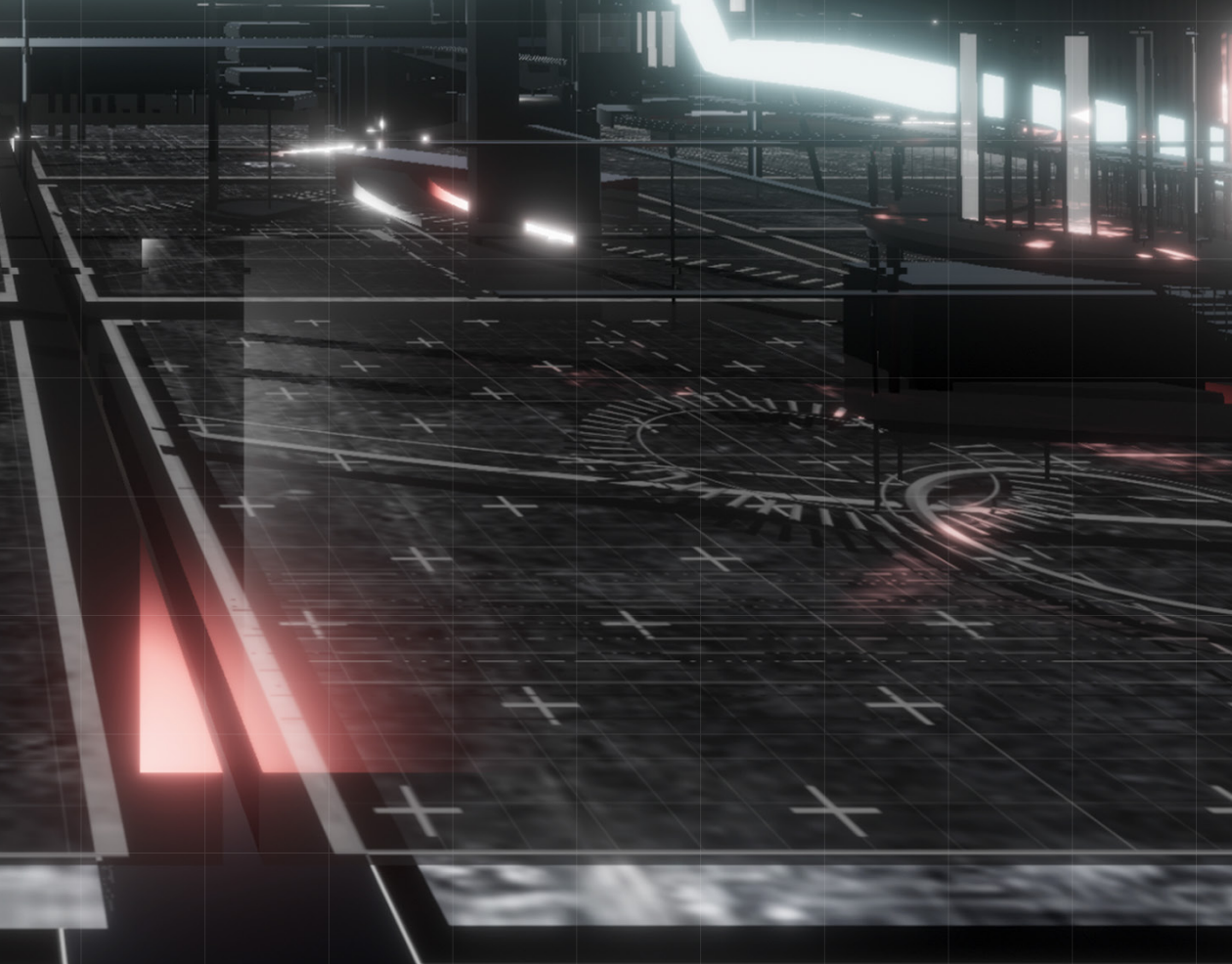


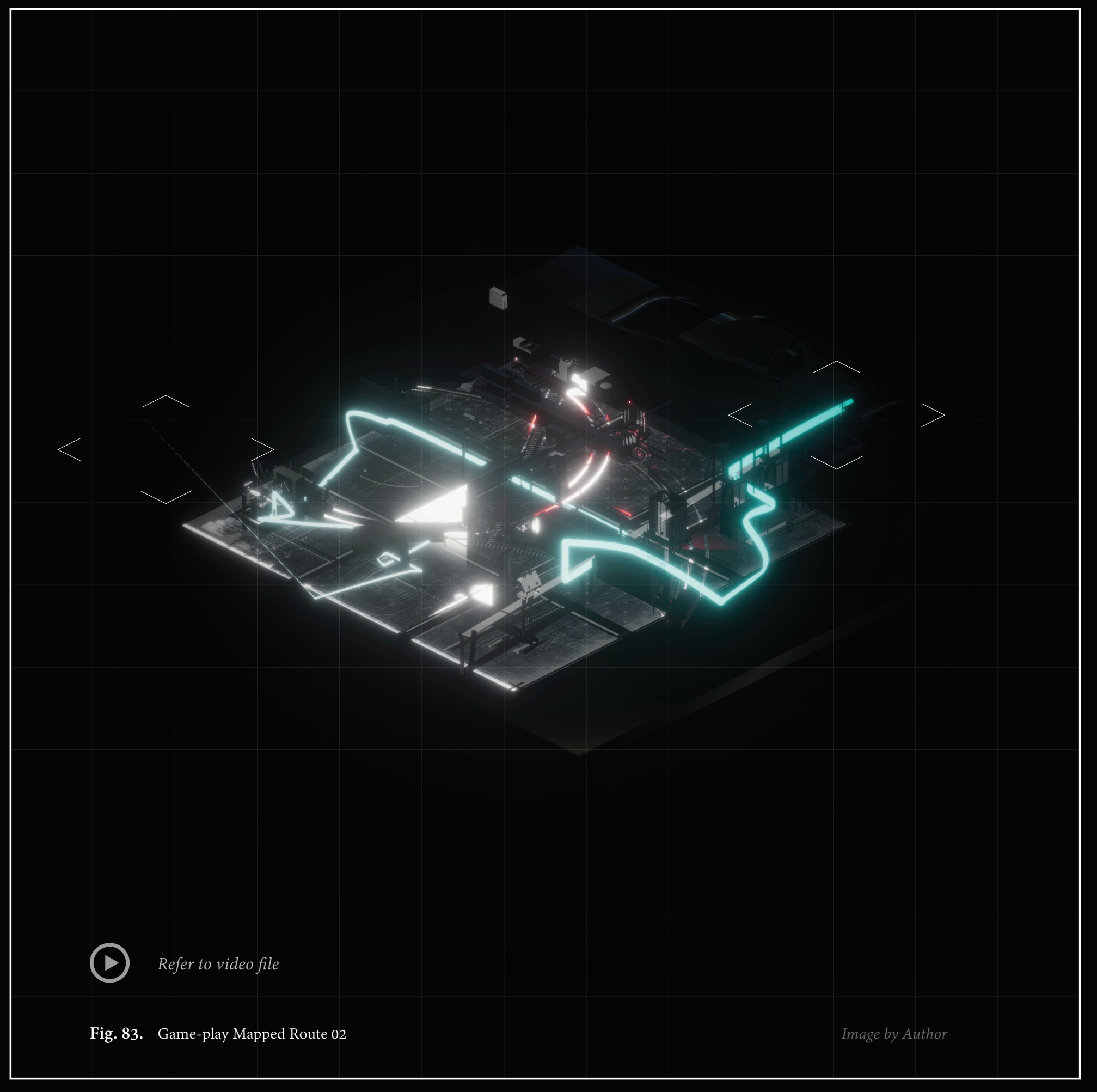




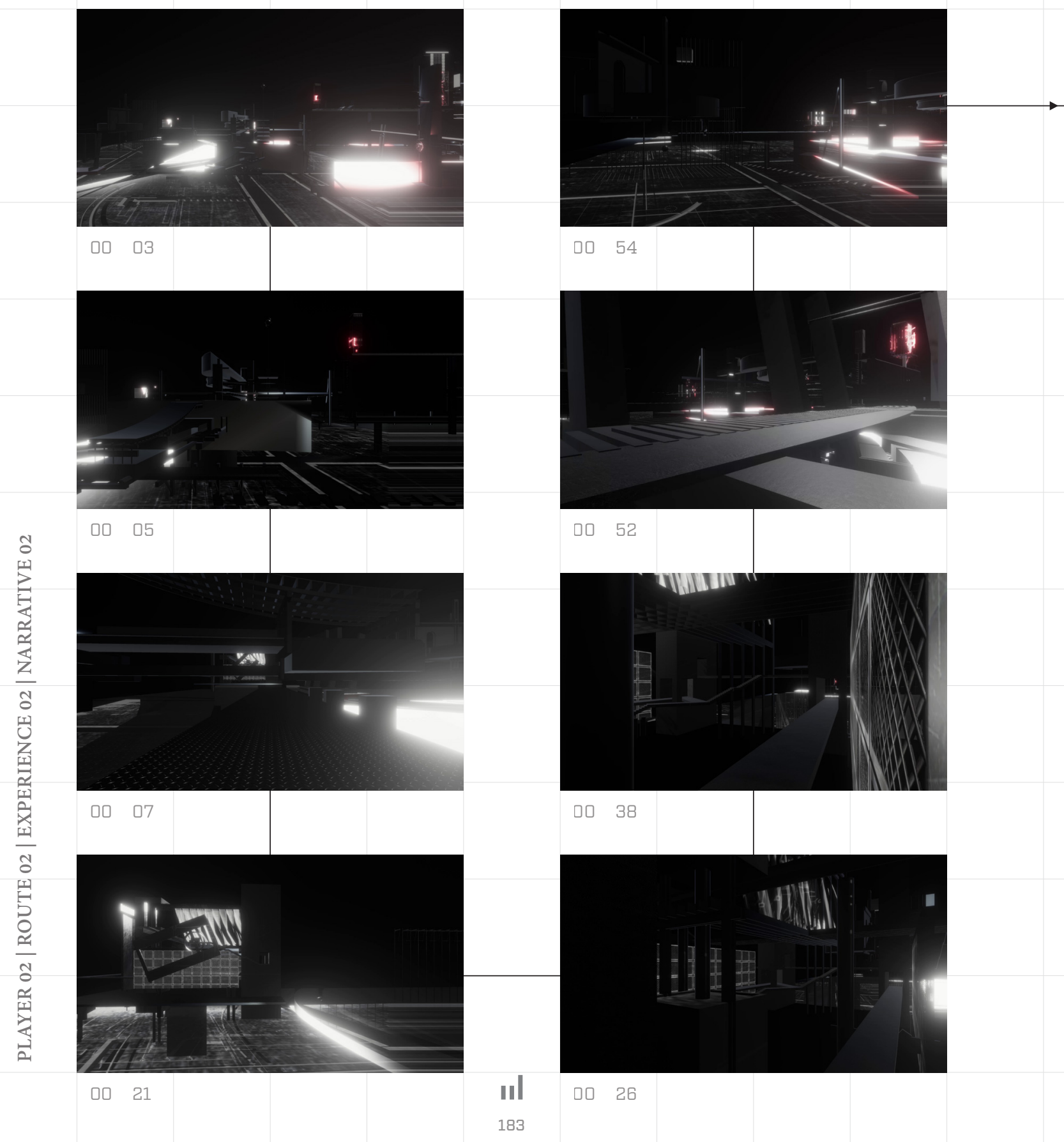



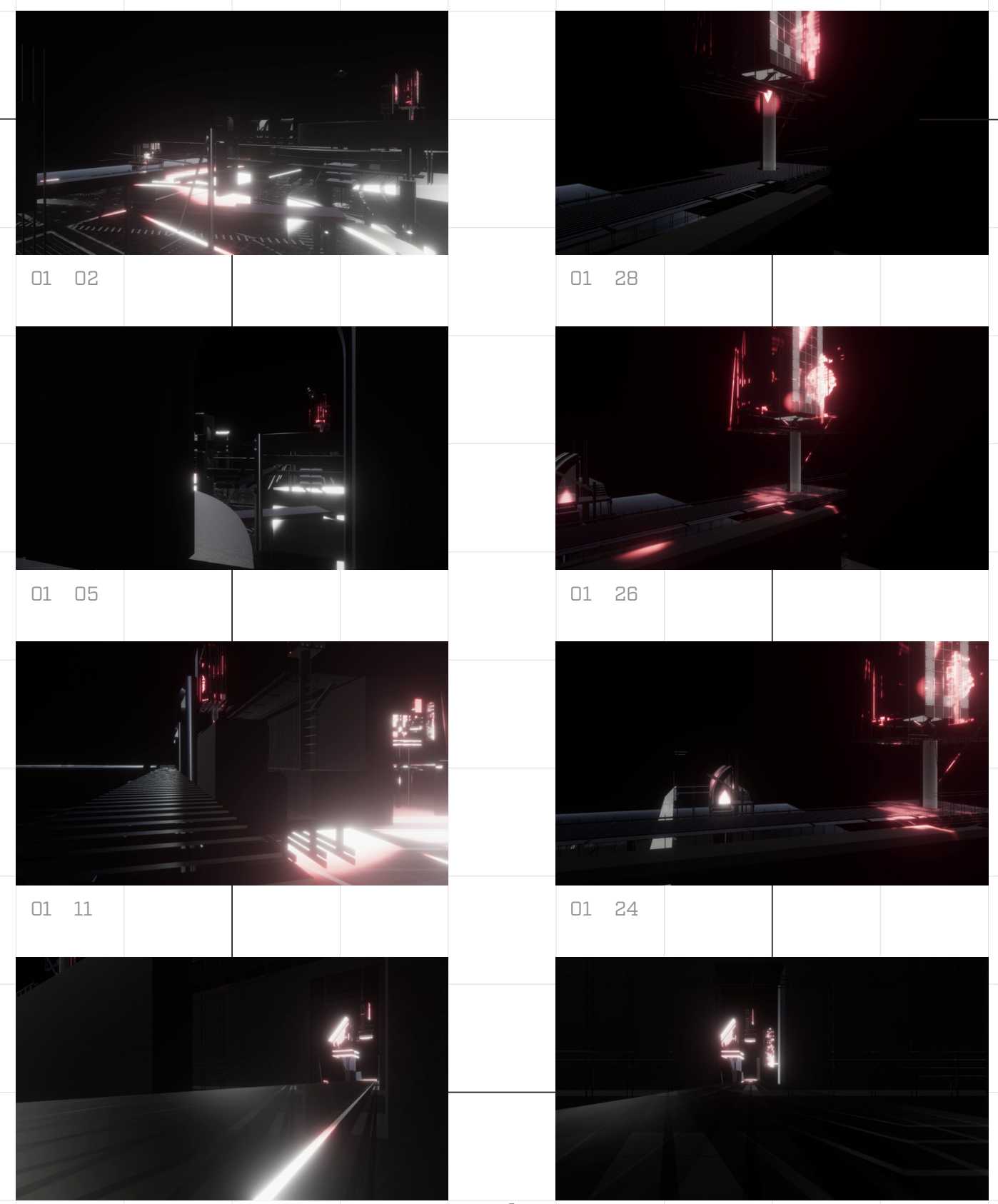

$01 \quad 14$ 

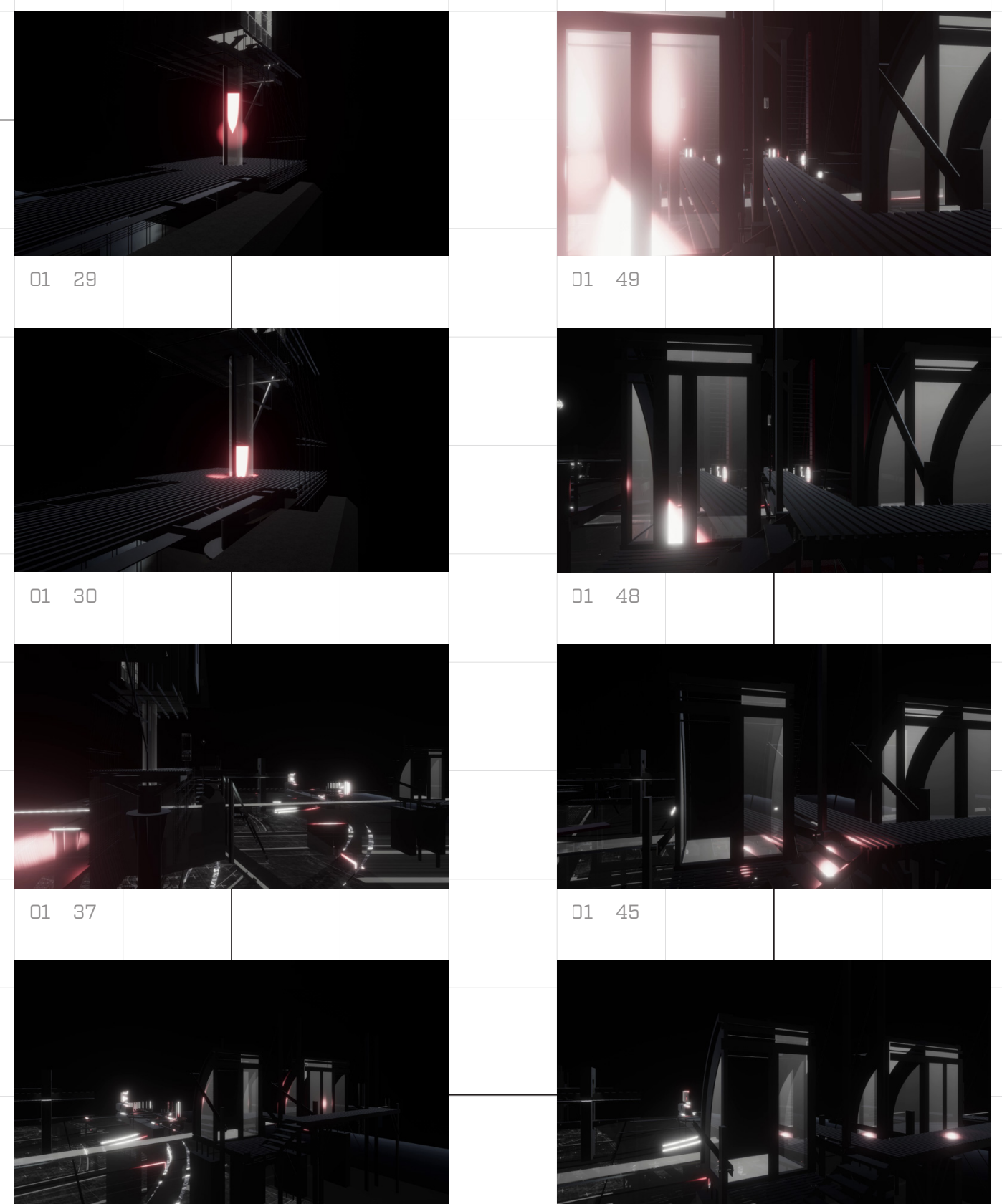

$01 \quad 40$

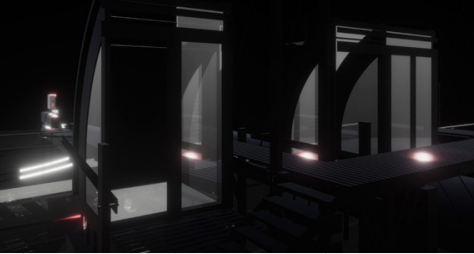




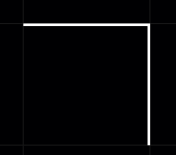

PLAYER 02 | ROUTE 02 | EXPERIENCE 02 | NARRATIVE 02 


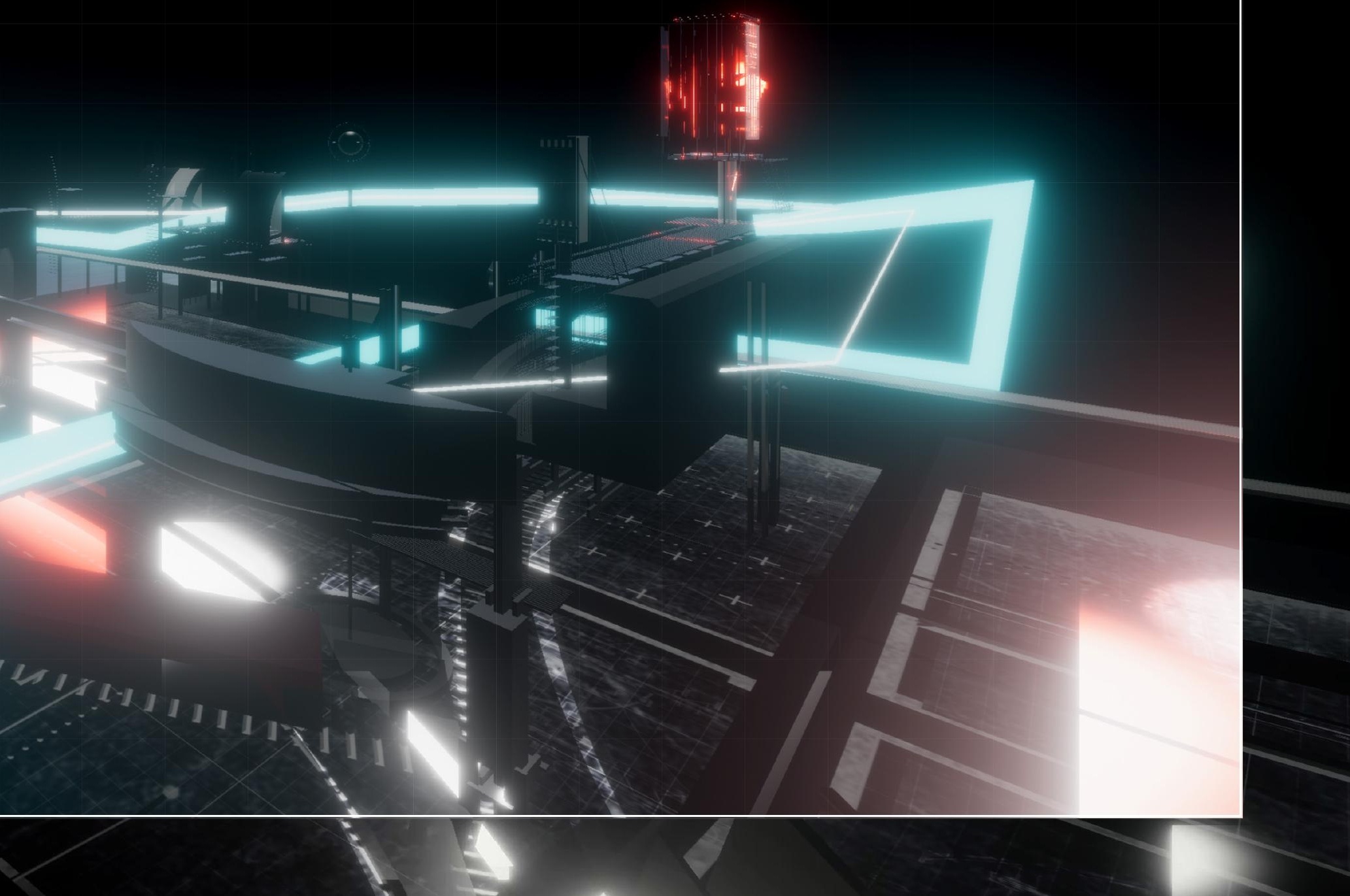




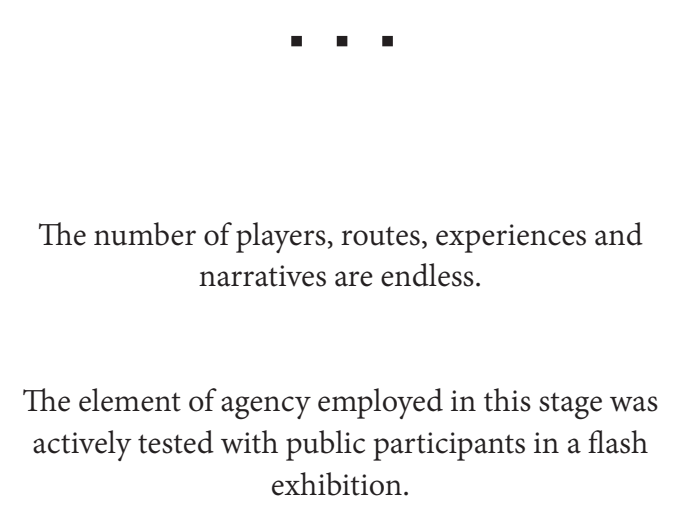




\section{Flash Exhibition}

@ Parsonson Architects

w/

Humbugaa

Friday 26th July

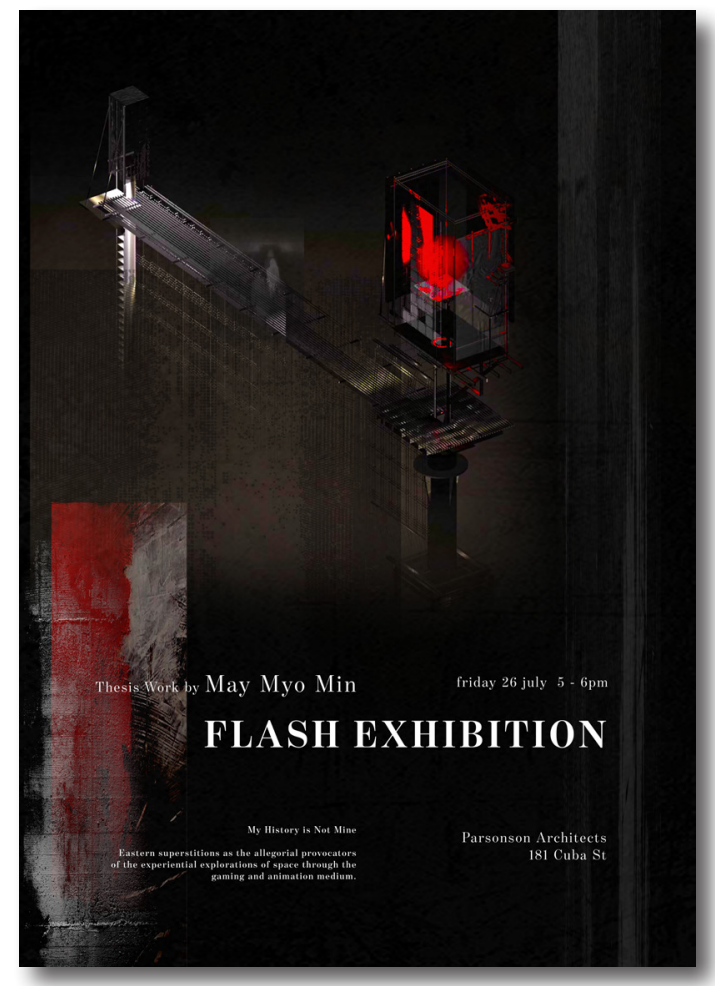

III 


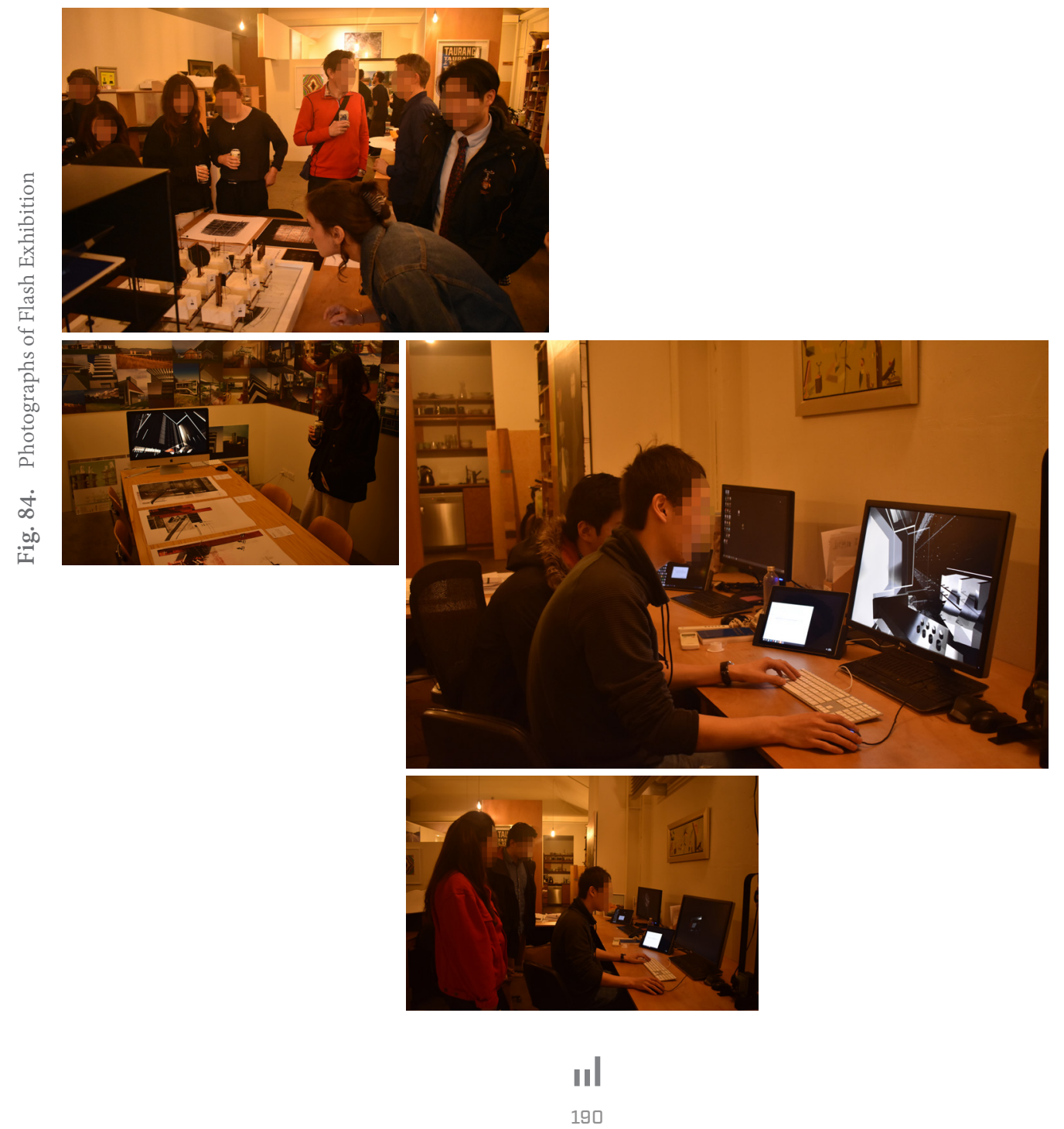



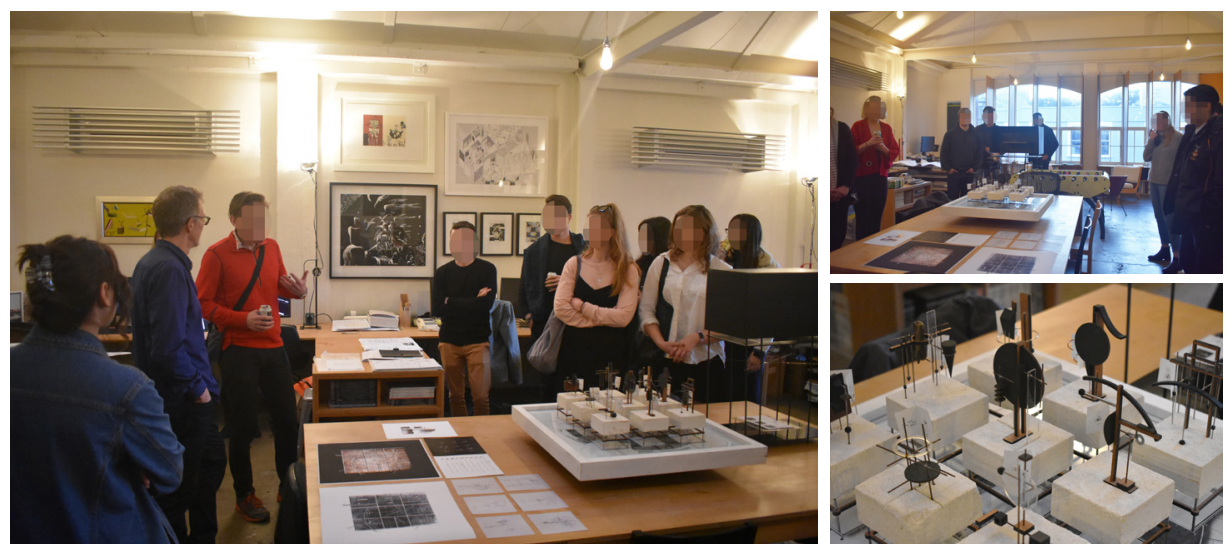

In the early conceptual stages of my design explorations, I had the privilege of exhibiting my thesis work at Parsonson Architects through Humbugaa's Flash Exhibition events.

The turn out was absolutely amazing and I received a lot of support, help and great feedback from friends and colleagues.

The event was organised to not only display my work, but it was also a great opportunity to test user participation in the game environment. It was an amazing opportunity that allowed me to observe the many different ways of how each participant chose to navigate through their own experience.
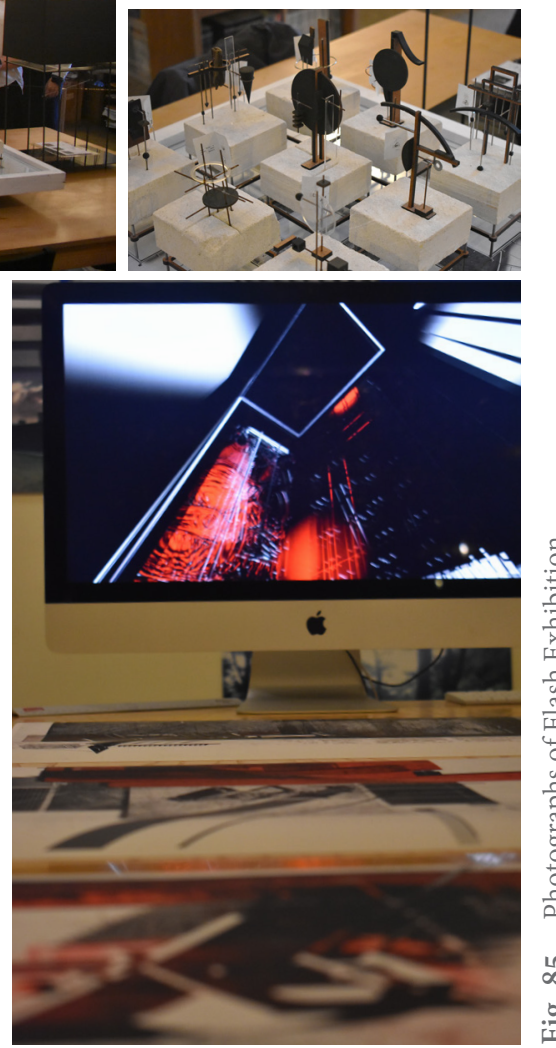

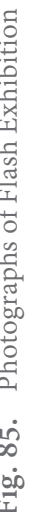


.. 
You too can be a player. You too can construct your own architectural narrative experience within this fabricated reality.

\section{G日a an}

PLAY GAME 


\section{Critical Reflection}

\section{REFLECTION ON DESIGN REVIEW 3: FEEDBACK AND DISCUSSIONS}

An extra set of explorations was discussed during the third review where I proposed for the construction of a physical model of the game space with the 9 interventions modelled, each divided into their movable kinetic parts around which participants could freely move.

The idea here was to introduce extra depth of agency for the participants to not only be able to have control over the paths they choose to navigate within the game environment, but to also have authority over the architecture itself and its constituent parts.

As the morphological nature of the interventions determines how each piece is read and interpreted, the alterations would allow the participant to construct and alter their own spatial experience along the way.

After a thorough discussion, reflection and debate with the help of the reviewers, we came to an agreement that this set of explorations was unnecessary. It was decided that it is beyond the scope of this thesis to investigate participatory design; the reason being that this project started from an autobiographical genesis, with strong personal connections to the artefacts and so the project needs to stand as a selfpositioning exercise within a controlled landscape.
This means that there needs to be a clear distinction of who the architect is and who the readers of the space are to ensure that the personalized elements of the artefacts are not lost through the influence of the users, but is rather enjoyed and interpreted by the users through their own perceptual understanding of a world constructed through the abstraction and interpretation of my lenses.

We come back to the heart of Lynn's poem where the lament strongly craves the identification of the unique self not influenced and wiped away by the authority figure.

I have not written my history

They have written it for me, those academics

They have written their own versions

-Lynn

This project positions itself as an invitation for users of all cultures and ages into a world that is mine, as a middle point, a meeting ground for negotiating and finding one's own meaning. 


\section{REFLECTION ON DEVELOPED DESIGN: DESIGN STAGE 3}

Stage 3 set out to explore how Digital Gaming Environments can be actively engaged in the architectural design methods and processes to enable agency to actively participate in the experiential narrative outcomes [RO 3].

The construct of the game is founded upon the theoretical framework based on Bruner's theory relating to narrative in literary fiction. While Design Stages 1 and 2 addressed 7 of Bruner's narrative attributes, Design Stage 3 used a virtual gaming environment to interrogate the remaining 3 narrative attributes that explicitly invoke agency: Intentional State Entailment, Normativeness and Context Sensitivity/Negotiability.

Design Stage 3 addressed Intentional State Entailment-the introduction of agency as a presumption of choice-through allowing players the freedom to navigate through the digital environment and interpret and respond to the spaces through their own individual perspectives.

Normativeness - the establishment of a norm that is breached and also understood in spatial context as the multiplicity of experiences never being identical to any two people-is addressed through the possibilities of endless experiences.
Context Sensitivity/Negotiability - the notion that ideals and meanings are not imposed but are rather negotiated through one's own cultural process-is addressed through the notion of space as a subjective experience where all ages and cultures can construct their own pathways and their own meaning in interpretation of the space.

The other 7 narrative features posited by Bruner were interrogated and integrated in Design Stages 1 and 2 . The ten features together all contribute to the assemblage of the collective architectural narrative experience within the game environment.

1. Diachronicity

2. Particularity

3. Intentional State Entailment

4. Hermeneutic Composability

5. Canonicity and Breach

6. Referentiality

7. Genericness

8. Normativeness

9. Context Sensitivity / Negotiability

10. Narrative Accrual 
Diachronicity is addressed through the time-sensitive animated interventions developed through Design Stage 2. Their formal and spatial narratives shifting as events unfolding over time provides the conceptual framework around which Design Stage 3 is actualised.

Particularity is addressed through the unique constituent parts of the 9 interventions-the kinetic and stationary elements that make up each piece are bound by their own time and space.

Hermeneutic Composability is addressed through synecdoche-the meaning of symbolic forms in the abstracted autonomous neutral objects of the artefacts that develops into the 9 interventions. They deviate away from conventional norms, with no culturally imposed ideal, no defined programs and no defined "truths".

Canonicity and Breach is addressed through the innovative and unconventional methodology that prioritises the experiential narrative qualities as the tool of enquiry.

Referentiality is addressed through the construct of a virtual autonomous world unbound by realworld constraints, but one that makes references to real-world concepts, truths and ideas.
Genericness is addressed through the removal of culturally imposed ideals and the denotation of concepts to arrive at abstracted architectural notions to drive the design towards a genre that is universally understandable and not exclusively referential to a certain culture.

Narrative Accrual is addressed through the uniting of the 9 interventions to form a collective whole-the game environment, where the merging of 9 complex and distinct narratives contributes to the layering of concepts.

The developed design stage tested the integration of Bruner and Ganoe's narrative theories, within the framework of Haralambidou's allegorical architectural project to argue that an architectural narrative experience can be constructed in a similar way to a fictional narrative. In the developed design outcome, the construct of the architectural story is built upon a complex relationship between 9 interventions, and the participant is invited through the construct of the game to assert their own interpretation of their experience of the space. This was tested in the public setting of a flash exhibition, where visitors were invited to play the game, and their experiences were observed. 
The complexity of the 9 interventions allowed the collective experience to be layered and offer rich opportunities for exploration and navigation. While they collectively created a world of complex narrative relationship, the transitioning between one intervention to the other was not quite as successful as their individually animated schemes. Also, the use of a 9-square grid format privileged the centre unit, rather then enabling all nine to be sited non-hierarchically.

The link drawing in Fig. 76 on page 165 attempts to synthesise and organise the 9 interventions as a collective set. It does so successfully to an extent that the 9 interventions are bound to a system and it attempts to employ different methods of linking and referencing their similarities_-such as transitional elements, elements of light projection, rotational pivoting elements and formal properties influenced by the drawings of the links. However, the organising system does not make reference to all the traits each intervention shares, as it became too complex to organise. This weakness is apparent only in the organising of the collective whole-it does not highly affect the spatial narrative experience that the investigation set out to achieve through $\mathrm{RO}$.

The second weakness apparent through the complexity of the collective field is that the opportunities set up for event triggers in the animated explorations of Design Stage 2 aren't carried out successfully in this stage. The setting up of events for players to trigger proved too time consuming and required extensive technical coding and scripting - a task that was considered to be too complex for the scope of this investigation. An informed compromise ultimately had to be made to meet time constraints: the element of agency is addressed through the freedom of player navigation and interpretation of space through personalised perception and experience rather than the players having a choice in altering the space themselves.

Again, this presents the issue addressed earlier where this is justified as the project takes an autobiographical stance, in which others can assert their own unique interpretation of a world constructed through my personalised lenses. 
曲 
5. Conclusions \& Critical Reflections

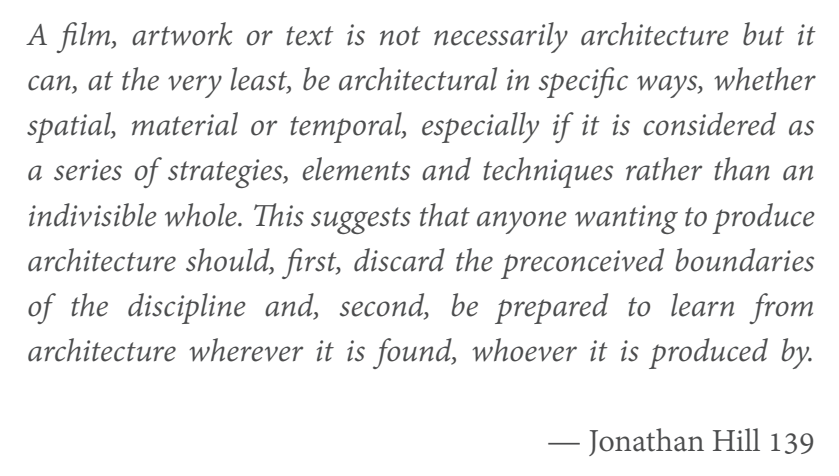


In this modern era driven by globalisation, we are constantly searching for ways of retaining valuable cultural elements to prevent them from being diminished or lost with the traditional dominance of Westernideals andEurocentricconstructs. Thisdesign research-led investigation sought possible solutions to mitigating the loss of unique cultural identity in contemporary architecture. The investigation does this by collecting culture-specific superstitious beliefs and abstracting and reinterpreting their allegorical symbolic meanings within the construct of an architectural experiential narrative.

This thesis explored the following two principal research questions:

1. How can experiential cultural artefacts be engaged as a conceptual framework to generate an allegorical architectural project?

2. How can the digital gaming interface be used to help architectural design methods better explore the experiential as a design generator?

It was essential that this issue be addressed and explored through design, as the questions are focused upon notions of cultural concepts shifting into an architectural narrative experience. The principal aim of the investigation was to test an architectural design method that prioritises the experiential and challenges some of the 'norms' within which Eurocentric modern architecture have been traditionally situated. The formal outcomes of the preliminary designs, although other-worldly, attempt to move towards a way of generating architectural concepts derived from unique cultural elements. They seek possible approaches to conceiving and testing a new and contemporary process for retaining important cultural values through architectural allegory.

To frame and test the conceived framework through the properties of a spatial experience, the design methodology employed a digital gaming environment-a mode of enquiry that provides some of the characteristics that can simulate spatial experience, without having to build a 1:1 architectural model for testing.

The scope of the project limits the exploration of the spatial experience, while the digital realm removes some of the constraints of real-world logic and physics. This methodology was selected to enable architectural notions to be re-defined and re-interpreted in ways that challenge and push the boundaries of the norms of architectural understanding.

With the thesis's constraints relating to time and available resources, the methodology is appropriate for testing spatial experience through the merging of a collection of interventions. This required extensive experimentation that cannot readily be tested outside of a digital interface, especially when considering issues of agency. The digital software offers abilities of simulation and repeatable experiments to take place without incurring substantial costs. 
This thesis investigation explored the Allegorical Architectural Project as a framework within which architectural and spatial/experiential concepts can be reinterpreted and redefined. Architectural theorist Penelope Haralambidou critically reflects upon three principal traits of allegory in architecture:

\section{FIGURATIVE GEOMETRIES}

(allegorical architecture)

2. PROGRESS \& JOURNEY

(narrative architecture)

3. INVITATION FOR INTERPRETATION

(experiential architecture)

These traits were tested through design in relation to the narrative theories of Jerome Bruner and Cathy Ganoe, in the exploration and testing of a design-based theoretical framework for architectural allegory.

If the study were to be continued, the implication of the adjacencies of these architectural artefacts could be examined, in relation to how together they are experienced as integrated spaces, thresholds and framing events. In addition, more participants could be invited to journey through the game environment, their pathways documented and their responses qualitatively analysed. The Flash Exhibition was a very useful opportunity that gathered many participants to partake in the exploration of the game environment; however, the in-depth recording and analysis of their responses was outside the scope of this investigation. There are endless possibilities, endless interpretations and endless journeys to be experienced by new participants.
My history has just begun

I am going to write my own history.

$$
\text { -Zeyar Lynn }
$$

There are as many versions of a story as there are listeners. There are as many experiences as there are players.

It is not the voice that commands the story: It is the ear - Italo Calvino 135 

MY HISTORY IS NOT MINE 


\section{Cited Works}

Barthes, Roland. S/Z. [1st American ed.], Hill and Wang, 1974. Print.

Bergh, Wim H. J. "Icarus' Amazement, Or the Matrix of Crossed Destinies." The Lancaster Hanover Masque: John Hejduk: [... Produced to Accompany an Exhibition of the Lancaster Hanover Masque at the Centre Canadien d'Architecture in Montréal, from 1 April to 21 June 1992, by John Hejduk, Architectural Assoc. u.a., 1992, pp. 81-102. Print.

Bruner, Jerome. "The Narrative Construction of Reality." Critical Inquiry, vol. 18, no. 1, 1991, pp. 1-21. JSTOR. Web. 30 Jun. 2019.

Calvino, Italo. Invisible Cities. [1st ed.], Harcourt Brace Jovanovich, 1974. Print.

Clear, Nic. "Drawing Time." Architectural Design, vol. 83, no. 5, 2013, pp. 70-79. Wiley Online Library. Web. 20 Aug. 2019.

Cobley, Paul. Narrative. Routledge, 2001. Print.

Eco, Umberto. A Theory of Semiotics. Indiana University Press, 1976. Print.

Faure, Elie. "De la ciniplastique." L'arbre d’Éden, Crès, 1922. Print.

Fletcher, Angus. Allegory, the Theory of a Symbolic Mode. Cornell University Press, 1964. Print.

Ganoe, Cathy J. "Design as Narrative: A Theory of Inhabiting Interior Space." Journal of Interior Design, vol. 25, no. 2, 1999, pp. 1-15. Wiley Online Library. Web. 3 Sep 2019.

Haralambidou, Penelope. "The Fall: The Allegorical Architectural Project as a Critical Method." Critical Architecture, Sept. 2007. Web. 30 Jun. 2019.

Hays, K. Michael. Hejduk's Chronotope. 1st ed., Princeton Architectural Press, 1996. Print. 
Hejduk, John. Riga, Vladivostok, Lake Baikal: A Work. Rizzoli, 1989. Print.

Hejduk, John, and Architectural Association (Great Britain). Victims: A Work. Architectural Association, 1986. Print.

Hill, Jonathan. Occupying Architecture : Between the Architect and the User. Routledge, 2005. Web. 3 Sep 2019.

Lee, Christina. “We'll Always Have Hong Kong’: Uncanny Spaces and Disappearing Memories in the Films of Wong Kar Wai." Violating Time: History, Memory, and Nostalgia in Cinema, Continuum International Publishing Group, 2008, pp. 124-41. Web. 2 Dec. 2019.

Lynn, Zeyar. "My History Is Not Mine." Bones Will Crow: 15 Contemporary Burmese Poets, edited by Ko Ko Thett and James Byrne, Arc Publications, 2012, pp. 148-49. Print.

Murray, Edward L. "Imagination and Phenomenological Thought." Imagination and Phenomenological Psychology, edited by Edward L. Murray, Duquesne Pres, 1987. Print.

Pallasmaa, Juhani. The Architecture of Image: Existential Space in Cinema. 2nd ed., Rakennustieto, 2007. Print.

Psarra, Sophia. Architecture and Narrative: The Formation of Space and Cultural Meaning. Routledge, 2009. Print.

Tschumi, Bernard. Cinegram Folie: Le Parc de La Villette. Princeton Architectural Press, 1987. Print.

---. The Manhattan Transcripts. Academy Editions, 1981. Print.

---. Tschumi Parc de La Villette. Artifice, 2014. Print.

Vidler, Anthony. The Architectural Uncanny: Essays in the Modern Unhomely / Anthony Vidler. MIT Press, 1992. Print.

---. “The Explosion of Space: Architecture and the Filmic Imaginary.” Assemblage, no. 21, 1993, pp. 45-59. JSTOR. Web. 12 Oct. 2019. 


\section{Bibliography}

Bachelard, Gaston. The Poetics of Space. Beacon Press, 1994.

Baudrillard, Jean, and Francesco Proto. Mass, Identity, Architecture Architectural Writings of Jean Baudrillard. 2003.

Bordwell, David. Narration in the Fiction Film. University of Wisconsin Press, 1985.

Braid, Donald. "Personal Narrative and Experiential Meaning." The Journal of American Folklore, vol. 109, no. 431, 1996, pp. 5-30. JSTOR, doi:10.2307/541716.

Bryant, Rebecca. "On Critical Times: Return, Repetition, and the Uncanny Present." History and Anthropology, vol. 27, no. 1, Jan. 2016, pp. 19-31. Taylor and Francis+NEJM, doi:10.1080/02757206.2015.1114481.

Calleja, Gordon. Experiential Narrative in Game Environments. p. 9.

Chapman, Michael. (Re) Findings: Discovery and Memory in the Architecture and Legacy of Surrealism. p. 9.

Coates, Nigel. Narrative Architecture: Architectural Design Primers Series. John Wiley \& Sons, Incorporated, 2012.

ProQuest Ebook Central, http://ebookcentral.proquest.com/lib/vuw/detail.action?docID=1104729.

Cowan, Bainard. "Walter Benjamin’s Theory of Allegory." New German Critique, no. 22, 1981, pp. 109-22. JSTOR, JSTOR, doi:10.2307/487866.

Coyne, Richard. "The Embodied Architect in the Information Age." Arq: Architectural Research Quarterly, vol. 3, no. 2, June 1999, pp. 175-86. Cambridge Core, doi:10.1017/S1359135500001950.

Dunne, Anthony, and Fiona Raby. Speculative Everything: Design, Fiction, and Social Dreaming. MIT Press, 2013.

ProQuest Ebook Central, http://ebookcentral.proquest.com/lib/vuw/detail.action?docID=3339745.

Fineman, Joel. “The Structure of Allegorical Desire." October, vol. 12, 1980, pp. 47-66. JSTOR, JSTOR, doi:10.2307/778574.

Gaines, Elliot. "Communication and the Semiotics of Space." Journal of Creative Communications, vol. 1, no. 2, July 2006, pp. 173-81. DOI.org (Crossref), doi:10.1177/097325860600100203. 
Grassi, Valentina. "The Question of Reality: The Relationship Between the Real and the Unreal in Baudrillard and Morin." Italian Sociological Review, vol. 7, no. 1S, Oct. 2017. www.italiansociologicalreview.com, http://www.italiansociologicalreview.com/ojs/index.php?journal=ISR\&page=article\&op=view\&path\%5B\%5D=483.

Hawkes, Terence. "Review of Allegory: The Theory of a Symbolic Mode." Renaissance News, vol. 18, no. 4, 1965, pp. 340-42. JSTOR, doi: $10.2307 / 2858387$.

Hexmoor, Henry, et al. Agent Autonomy. Springer Science \& Business Media, 2012.

Holm, Lorens. Brunelleschi, Lacan, Le Corbusier: Architecture, Space and the Construction of Subjectivity / Lorens Holm. Routledge, 2010.

---. "Glimpsing the Real in Architecture, or The Sun Always Rises." Perspecta, vol. 42, 2010, pp. 11-16. JSTOR.

Ingarden, Roman. The Literary Work of Art: An Investigation on the Borderlines of Ontology, Logic, and Theory of Literature. Northwestern University Press, 1973.

Lee, Christina, editor. Violating Time: History, Memory, and Nostalgia in Cinema. Continuum International Publishing Group, 2008. DOI.org (Crossref), doi:10.5040/9781628929164.

Macleod, Suzanne, et al. Museum Making: Narratives, Architectures, Exhibitions. Routledge, 2012. ProQuest Ebook Central, http://ebookcentral.proquest.com/lib/vuw/detail.action?docID $=956902$.

McGregor, James. "The Architect as Storyteller: Making Places in John Hedjuk's Masques." Architectural Theory Review, vol. 7, no. 2, Nov. 2002, pp. 59-70. Crossref, doi:10.1080/13264820209478457.

Merleau-Ponty, Maurice. Phenomenology of Perception. Routledge, 2013. www.taylorfrancis.com, doi:10.4324/9780203720714.

Mitnick, Keith. Artificial Light: A Narrative Inquiry into the Nature of Abstraction, Immediacy, and Other Architectural Fictions. Princeton Architectural Press, 2008. ProQuest Ebook Central, http://ebookcentral.proquest.com/lib/ vuw/detail.action?docID $=3387413$. 
Mitsecherling, Jeff. Roman Ingarden's Ontology and Aesthetics. University of Ottawa Press, 1997. ProQuest Ebook Central, http://ebookcentral.proquest.com/lib/vuw/detail.action?docID $=653357$.

Quantrill, Malcolm. The Environmental Memory: Man and Architecture in the Landscape of Ideas. Schocken Books, 1987.

Radovic, Marko. Bernard Tschumi -Six Concepts Excerpt from Architecture and Disjunction CONCEPT I: Technologies of Defamiliarization.www.academia.edu, https://www.academia.edu/7600643/Bernard_Tschumi_-Six_Concepts_Excerpt_from_Architecture_and_Disjunction_CONCEPT_I_Technologies_of_Defamiliarization. Accessed 3 Sept. 2019.

Ricoeur, Paul. Time and Narrative. University of Chicago Press, 2010.

Spiller, Neil. Celebrating the Marvellous: Surrealism in Architecture. John Wiley \& Sons, Incorporated, 2018. ProQuest Ebook Central, http://ebookcentral.proquest.com/lib/vuw/detail.action?docID=5317898.

Tschumi, Bernard. Architecture and Disjunction. MIT Press, 1996.

Uysal, V. Şafak, and Levent Aridağ. "'Perform-Box': Towards an Architecture-of-Time." Performance Research, vol. 17, no. 5, Oct. 2012, pp. 119-29. Taylor and Francis+NEJM, doi:10.1080/13528165.2012.728452.

Vidler, Anthony. Warped Space: Art, Architecture, and Anxiety in Modern Culture / Anthony Vidler. MIT Press, 2000 . 



\section{Sources of Figures}

Fig. 3. Tschumi's Conceptual Notation Diagram models of Parc de la Villette

Tschumi, Bernard. Parc de la Villette Combinatorial models. 1983. 19 November 2019.

Web. <http://socks-studio.com/2014/12/29/the-combinatorial-models-of-the-folies/>

Fig. 4. Tschumi's Notation Diagram "The Fall”

Tschumi, Bernard. The Manhattan Transcripts. Academy Editions, 1981: V. Print.

Fig. 5. Tschumi's Conceptual Notation Diagram of Parc de la Villette

Tschumi, Bernard. Tschumi Parc de la Villette. Artifice, 2014: 59. Print.

Fig. 7. Hejduk's Masques: Painter-Studio A

Hejduk, John. Riga, Vladivostok, Lake Baikal: A Work. Rizzoli, 1989: 103. Print.

Fig. 8. Hejduk's Masques: Musician-Studio B

Hejduk, John. Riga, Vladivostok, Lake Baikal: A Work. Rizzoli, 1989: 103. Print.

Fig. 9. Hejduk's Masques: Security-Structure

Binet, Helene. John Hejduk's Victims: Dimension, Death \& Identity, “Security” at Christiania torg in Oslo in 1989. 1 January 2020.

Web. <https://www.architecturenorway.no/stories/other-stories/malmquist-on-hejduk-2009/>

Fig. 10. Hejduk's Masques: Clock/Collapse of Time

Hejduk, John. Riga, Vladivostok, Lake Baikal: A Work. Rizzoli, 1989: 75. Print.

Fig. 11. Hejduk's Masques

Ngam, Adrianne. The John Hejduk Equivalent to the Utah Teapot. 20 August 2017.

Web. <www.adriannengam.com/home/2017/8/22/the-john-hejduk-equivalent-to-the-utah-teapot>

Fig. 12. Hejduk's Victims Drawing Compilations of Masques coexisting as a collective whole

Hejduk, John. Victims. 1986. 1 January 2020.

Web. <http://traverza.blogspot.com/2012/10/architekt-john-hejduk.html> 
Fig. 14. Clear's student's Chronogram: Danai Surasa, Panic Room space-time analysis chronogram.

Danai Surasa, Panic Room space-time analysis chronogram in Clear, Nic. "Drawing Time."

Architectural Design, vol. 83, no. 5, 2013, pg. 77. Wiley Online Library. Web. 20 Aug. 2019.

All unattributed figures belong to the author

Note: Digital components from online sources, not by the author have been integrated into the collaging, 3D modelling, animation and game environment in this work. 